\title{
Ratio of Pion Kaon Production in Proton Carbon Interactions
}

\author{
A dissertation presented \\ by \\ Andrey V Lebedev \\ to \\ The Department of Physics \\ in partial fulfillment of the requirements \\ for the degree of \\ Doctor of Philosophy \\ in the subject of \\ Physics \\ Harvard University \\ Cambridge, Massachusetts
}

May 2007 
(C)2007 - Andrey V Lebedev

All rights reserved. 


\title{
Ratio of Pion Kaon Production in Proton Carbon Interactions
}

\begin{abstract}
The ratio of pion-kaon production by $120 \mathrm{GeV} / c$ protons incident on $\Lambda_{\text {carbon target }}$ is presented. The data was recorded with the Main Injector Particle Production experiment at Fermi National Accelerator Laboratory. Production ratios of $K^{+} / \pi^{+}, K^{-} / \pi^{-}, K^{-} / K^{+}$, and $\pi^{-} / \pi^{+}$are measured in 24 bins in longitudinal momentum from 20 to $90 \mathrm{GeV} / c$ and transverse momentum up to $2 \mathrm{GeV} / c$. The measurement is compared to existing data sets, particle production Monte Carlo results from FLUKA-06, parametrization of protonberyllium data at $400 / 450 \mathrm{GeV} / c$, and ratios measured by the MINOS experiment on the NuMI target.
\end{abstract}




\section{Contents}

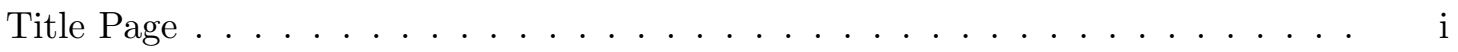

Abstract ...............................

Table of Contents . . . . . . . . . . . . . . . . . iv

List of Figures . . . . . . . . . . . . . . . . . . . . vi

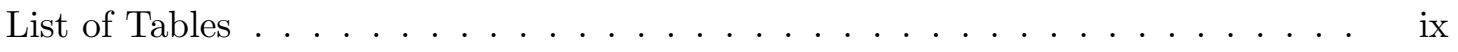

Acknowledgments . . . . . . . . . . . . . . . . . $\mathrm{x}$

Dedication .................................

1 Introduction 1

2 MIPP Detectors $\quad 5$

2.1 Tracking . . . . . . . . . . . . . . . . . . 5

2.2 Particle Identification . . . . . . . . . . . . . . . . . . . 10

2.3 Analysis Magnets . . . . . . . . . . . . . . . . . . 18

3 MIPP Trigger 20

3.1 Particle Identification . . . . . . . . . . . . . . . . . . . 20

3.2 Beam Cherenkov Operation . . . . . . . . . . . . . . . . 21

3.3 Interaction Trigger . . . . . . . . . . . . . . . . . . 23

3.4 End of Spill and Calibration Triggers . . . . . . . . . . . . . 25

3.5 Veto Counter . . . . . . . . . . . . . . . . . . 25

3.6 NuMI Trigger . . . . . . . . . . . . . . . . . . 26

4 Data Acquisition System $\quad 28$

4.1 VME and CAMAC Interfaces . . . . . . . . . . . . . . . 30

4.2 Online Software . . . . . . . . . . . . . . . . . . . . 33

4.3 Slow Controls . . . . . . . . . . . . . . . . . . . . 39

5 Beamline $\quad 41$

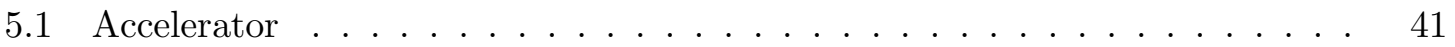

5.2 Primary Beam . . . . . . . . . . . . . . . . . . 43

5.3 Secondary Beam . . . . . . . . . . . . . . . . . . 43 
6 Event Reconstruction $\quad \mathbf{5 0}$

6.1 Track Fitting . . . . . . . . . . . . . . . . . 50

6.2 Wire Chambers . . . . . . . . . . . . . . . . . . . 54

6.3 Time Projection Chamber . . . . . . . . . . . . . . . . 59

6.4 Global Tracking . . . . . . . . . . . . . . . . . . . . . 60

6.5 Vertex Reconstruction . . . . . . . . . . . . . . . . . 63

6.6 Particle Identification . . . . . . . . . . . . . . . . . . 68

7 Detector Calibration and Alignment $\quad \mathbf{7 8}$

7.1 Event Time . . . . . . . . . . . . . . . . . . . . . 78

7.2 Drift Chamber Time Offsets . . . . . . . . . . . . . . . . . 81

7.3 Chamber Alignment . . . . . . . . . . . . . . . . 85

7.4 Tuning Magnetic Field . . . . . . . . . . . . . . . . . . . . 90

7.5 TPC Electron Drift . . . . . . . . . . . . . . . . . . 94

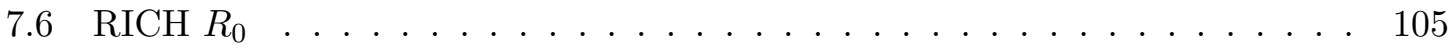

8 Monte Carlo Simulation $\quad 107$

8.1 Time Projection Chamber Digitization . . . . . . . . . . . . . 107

8.2 Wire Chamber Digitization . . . . . . . . . . . . . . . . . . 109

8.3 RICH Hits and Digitis . . . . . . . . . . . . . . . . . 110

9 Data Analysis $\quad 111$

9.1 Data Binning . . . . . . . . . . . . . . . . . . . . 111

9.2 Pileup Removal . . . . . . . . . . . . . . . . . . . . . . . 113

9.3 Primary Vertex Selection _. . . . . . . . . . . . . . . . 117

9.4 Interaction Trigger Efficiency . . . . . . . . . . . . . . . . . 120

9.5 Particle ID Variable . . . . . . . . . . . . . . . . . . . . . 121

9.6 Corrections from Monte Carlo Simulation . . . . . . . . . . . . . 126

9.7 Corrections and Errors . . . . . . . . . . . . . . . . . . . . . 132

10 Results $\quad 137$

10.1 Production Ratios . . . . . . . . . . . . . . . . 137

10.2 Comparison to Existing Data . . . . . . . . . . . . . . . 137

10.3 Comparison to MINOS Measurements . . . . . . . . . . . . . 145

10.4 Discussion and Outlook . . . . . . . . . . . . . . . 147

$\begin{array}{ll}\text { Bibliography } & 148\end{array}$ 


\section{List of Figures}

1.1 Summary of hadronic production data available in $2001 \ldots \ldots$. . . . . . . . 2

1.2 Uncertainties in neutrino flux prediction . . . . . . . . . . . . 3

1.3 MINOS muon neutrino (left) and electron neutrino (right) fluxes and the fraction of neutrinos produced in kaon decays. . . . . . . . . . . . . . . 3

2.1 Schematic of the MIPP spectrometer . . . . . . . . . . . . 6

2.2 Schematic view of the TPC volume and pad plane . . . . . . . . . . 9

2.3 Schematic of T00 and T01 construction . . . . . . . . . . . . . . 12

2.4 Schematic of RICH electronics setup. . . . . . . . . . . . . . . . 14

2.5 Electromagnetic calorimeter front view. . . . . . . . . . . . . 16

2.6 Hadron calorimeter front view . . . . . . . . . . . . . . . . . . . . . . 17

2.7 Projections of the JGG field map . . . . . . . . . . . . . . . . 18

2.8 Projections of the Rosie field map . . . . . . . . . . . . . . . . . . 19

3.1 Time of flight difference from $\beta=1$ particle in T00 and T01 counters . . . 22

3.2 Summary of upstream beam Cherenkov pressure curves with nitrogen . . . 23

3.3 Trigger particle tag purity as measured by the RICH . . . . . . . . . . . . 24

3.4 NuMI trigger logic . . . . . . . . . . . . . . . 26

4.1 Schematic of data acquisition setup . . . . . . . . . . . . . . . . 29

5.1 Schematic of the FNAL accelerator complex . . . . . . . . . . . . . 42

5.2 Analysis of the initial beamline setup . . . . . . . . . . . . . . 45

5.3 Simulation of tracking of particles through initial beamline setup . . . . . . 46

5.4 Solution for reversed beamline setup . . . . . . . . . . . . . . 48

5.5 Simulation of tracking of particles using reversed-beam solution . . . . . . . 49

6.1 Sketch of wire plane geometry. . . . . . . . . . . . . . . 51

6.2 Matching of DC123 and DC4/PWC56 segments in the center of Rosie . . . 58

6.3 Distribution of $\chi^{2}$ per degree of freedom and number of clusters in chamber

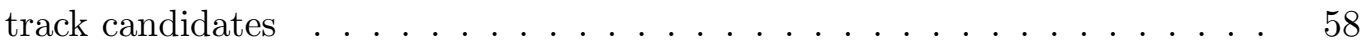

6.4 Selection of tracks for TMiniut fitter and resulting track momenta . . . . . 60

6.5 Matching of TPC tracks to chamber track candidates . . . . . . . . . . 62

6.6 Matching of tracks at the six chambers . . . . . . . . . . . 63 
6.7 Deterministic annealing filter weight functions for vertex finder . . . . . . 64

6.8 Number of $z$ scans required for a vertex constrained fit . . . . . . . . . . 67

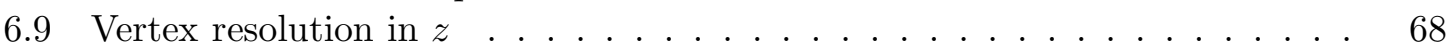

6.10 Mean RICH ring radii and RMS of light distribution . . . . . . . . . . . 70

$6.11 \mathrm{CO}_{2}$ refractive index and Hamamatsu PMT efficiency versus light wavelength 70

6.12 Effect of signal region on RICH ring fits . . . . . . . . . . . . . . 72

6.13 RICH ring shift from prediction . . . . . . . . . . . . . . 73

6.14 Ripples in RICH ring radius . . . . . . . . . . . . . . . . . . . 74

6.15 Dependence of amplitude of RICH ring radius ripples . . . . . . . . . 75

6.16 A $10 \%$ improvement in radius width of high momentum pions is obtained by removing ripple corrections through parametrized equations. . . . . . . . 76

6.17 Distribution of fitted RICH ring radii and computed mass squared . . . . . 77

7.1 Time of flight difference from $\beta=1$ particle for a straight line particle from the target to time of flight wall. . . . . . . . . . . . . . 79

7.2 Cable delay drift in $\mathrm{T} 0$ counters . . . . . . . . . . . . . . . . . 80

7.3 Time slewing due to charge on the T0 PMT's . . . . . . . . . . . . . . 82

7.4 Comparison of time difference resolution with and without time slewing cor-

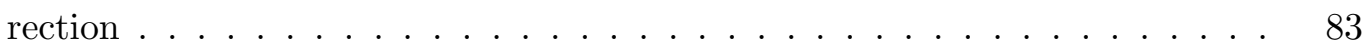

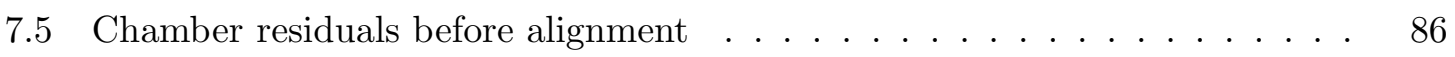

7.6 Chamber residuals after alignment . . . . . . . . . . . . . . . 87

7.7 DC123 z-alignment results . . . . . . . . . . . . . . . . . . . 88

7.8 DC123 alignment results with corrected DC3 angle . . . . . . . . . . . 89

7.9 Dependence of DC123 alignment constants on beam momentum . . . . . . 91

7.10 Dependence of DC4 and PWC56 alignment constants on beam momentum 92

$7.11 B_{x} / B_{z}$ rotation using chambers . . . . . . . . . . . . . . 93

7.12 Ratios of $B_{x} / B_{y}$ and $B_{z} / B_{y}$ in the magnets . . . . . . . . . . 93

7.13 Alignment summary versus run with geometry and magnetic field corrections 95

7.14 Alignment summary versus beam momentm with geometry and magnetic field corrections . . . . . . . . . . . . . . . 96

7.15 Dependence of TPC $x$-residual versus $z$ slope on $B_{x} / B_{z}$ rotation . . . . . 97

$7.16 B_{x} / B_{z}$ ratio in the magnets before and after correction . . . . . . . . . 98

$7.17 \mathrm{P} 10$ drift velocity measurements . . . . . . . . . . . . . . . . 99

7.18 Measured TPC trigger time offset versus run number . . . . . . . . . . . . 100

7.19 Measured TPC drift velocity versus run number . . . . . . . . . . . . . 101

7.20 Projections of $120 \mathrm{GeV} / c$ track hits . . . . . . . . . . . . . 103

7.21 Angle between the vertical and horizontal field component in the JGG . . . 104

7.22 RICH ring radius dependence on $\mathrm{CO}_{2}$ density . . . . . . . . . . . 106

9.1 RICH geometric acceptance . . . . . . . . . . . . . . . . . . . . . 112

9.2 Charged hadron fluxes simulated by FLUKA-06 . . . . . . . . . . . . . . 112

9.3 Expected number of $\pi^{+}$in bins selected for measurement . . . . . . . . 115

9.4 Momentum and $d E / d x$ in the TPC of uninteracted protons . . . . . . . 116

9.5 Distribution of variables used to remove pileup . . . . . . . . . . . . 118

9.6 Distribution of secondary track times on pileup events . . . . . . . . . . . 119 
9.7 Effect of pileup clean up on TPC $d E / d x$ and calorimeter energy $\ldots \ldots$. . 119

9.8 Vertex $z$ of $2 \%$ carbon and target out data . . . . . . . . . . . . 120

9.9 Interaction trigger efficiency . . . . . . . . . . . . . . . . . . 121

9.10 Momentum spectrum enhancement under assumptions of different trigger efficiencies . . . . . . . . . . . . . . . . . . . 122

9.11 Computed mass squared versus $p_{z}$ in different $p_{t}$ bins . . . . . . . . . 123

9.12 Computed Monte Carlo mass squared versus $p_{z}$ in different $p_{t}$ bins . . . . . 124

9.13 Data-Monte Carlo comparison of particle ID variable $\alpha$ for positives . . . . 127

9.14 Data - Monte Carlo comparison of particle ID variable $\alpha$ for negatives . . . 128

9.15 Monte Carlo signal and background in bin $(0,0) \ldots \ldots \ldots$

9.16 Occupancy of positive pions and kaons in Monte Carlo . . . . . . . . . . . 130

9.17 Occupancy of negative pions and kaons in Monte Carlo . . . . . . . . . . . 131

9.18 Reconstructed Monte Carlo to true Monte Carlo comparison . . . . . . . . 132

9.19 Effect on the ratios from interaction trigger efficiency . . . . . . . . . . . 133

9.20 Effect on the ratio from $5 \%$ change in target out scale factor . . . . . . . . . 134

9.21 Data/Monte Carlo background ratio . . . . . . . . . . . . . . . . . . 135

9.22 Systematic error from background subtraction . . . . . . . . . . . 136

10.1 Measured ratios compared to particle production simulated with FLUKA-06 on $2 \%$ carbon target . . . . . . . . . . . . . . . . . 138

10.2 Comparison to existing measurements . . . . . . . . . . . . . 143

10.3 Comparison to existing measurements . . . . . . . . . . . . . . . . 144

10.4 Comparison of the ratios on the NuMI target and the $2 \%$ target . . . . . 145

10.5 Comparison to Monte Carlo models and MINOS fitted ratios . . . . . . . . 146 


\section{List of Tables}

2.1 Summary of multi-wire chamber parameters . . . . . . . . . . . . . 7

2.2 Summary of chamber operation conditions . . . . . . . . . . . 7

4.1 Message classes implemented in run control. . . . . . . . . . . . . . . 34

$9.1\left(p_{z}, p_{T}\right)$ bins selected for ratio measurement $\ldots \ldots \ldots \ldots \ldots$

9.2 Trigger efficiency using beryllium and carbon data . . . . . . . . . . . . 120

9.3 Coefficients for conversion from $m^{2}$ into particle ID variable $\alpha \ldots \ldots$. . 125

$10.1 \pi^{-} / \pi^{+}$production ratio and errors . . . . . . . . . . . . . . . . . 139

$10.2 \mathrm{~K}^{-} / \mathrm{K}^{+}$production ratio and errors . . . . . . . . . . . . . . 140

$10.3 \mathrm{~K}^{+} / \pi^{+}$production ratio and errors . . . . . . . . . . . . . . . 141

$10.4 K^{-} / \pi^{-}$production ratio and errors . . . . . . . . . . . . . . . . . 142 
Acknowledgments 


\section{Chapter 1}

\section{Introduction}

Particle production is one of the classic phenomena studied by many experiments over the twentieth century. While many experiments focused on production of exotic particles, there were few high statistics measurements of inclusive production of pions, kaons, and protons. Some cross sections were measured with bubble chambers, but most high statistics measurements were done with single arm spectrometers $[2,11,1]$, and therefore did not provide complete coverage of space in longitudinal and transverse momentum (see Figure 1.1 $)^{1}$. As a result, hadronic production models like MARS[34] and FLUKA[23, 24] have limited data to be tuned with, and in some cases give significantly different results.

Scarcity of hadronic production data affects every neutrino experiment $[16,32$, $33,25]$ as accelerator made and atmospheric neutrinos come from the decay of pions and kaons produced in interactions of protons with matter. In the case of $\mathrm{NuMI}^{2} / \mathrm{MINOS}^{3}$ experiment, $120 \mathrm{GeV} / c$ protons are incident on a carbon target which is two interaction lengths long. The uncertainties in particle production models largely cancel in comparison of the near and far detector data, but calculation of neutrino flux with various hadronic production models differ by up to $30 \%$ (Figure 1.2). Since the near detector sees a line source of neutrinos and the far detector sees a point source of neutrinos, these uncertainties do not cancel completely, and the near/far detector comparison depends on the hadronic production model.

Recently, NA49 experiment at CERN published results of the measurement of

\footnotetext{
${ }^{1}$ We will refer to longitudinal momentum (along the direction of the incident particle) as $p_{z}$ and to transverse momentum (perpendicular to the direction of the incident particle) as $p_{T}$.

${ }^{2}$ Neutrinos at the Main Injector

${ }^{3}$ Main Injector Neutrino Oscillation Search
} 


\section{Low Energy Beam}

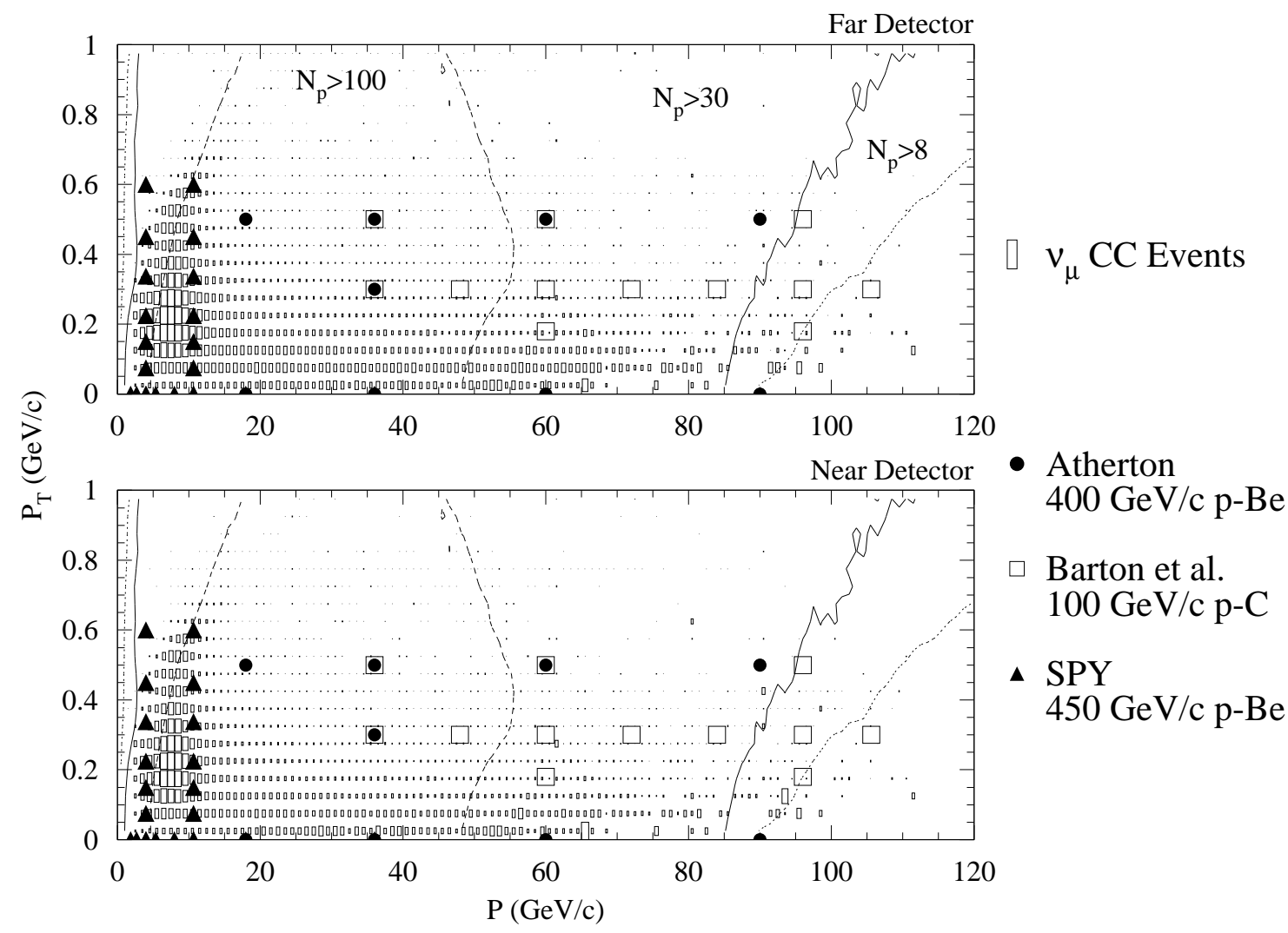

Figure 1.1: The plot from [8] shows the spectrum of pions which produce neutrinos in the MINOS far and near detectors. Superimposed are appropriately scaled data from single arm spectrometers[2, 11, 1].

charged pion production in proton carbon collisions at $158 \mathrm{GeV} / c$ [15], which is close to NuMI proton beam of $120 \mathrm{GeV} / c$. However, the secondary and tertiary particle production on the NuMI target is significant, so if one is to tune Monte Carlo models to better predict neutrino flux, it is essential to have the data of hadronic production of pions, kaons, and protons on carbon at lower momenta. Moreover, large fraction of high energy neutrinos come from decay of charged kaons (see Figure 1.3), so knowledge of kaon flux is necessary as well.

The Main Injector Particle Production (MIPP) experiment was designed to measure the total charged particle production of $\pi^{ \pm}, K^{ \pm}, p$, and $\bar{p}$ by tagged beam of pions, kaons, and protons on nuclear targets. Experiment is capable of selecting beam momentum from 5 to $85 \mathrm{GeV} / c$, or run with $120 \mathrm{GeV} / c$ proton beam from the Main Injector 

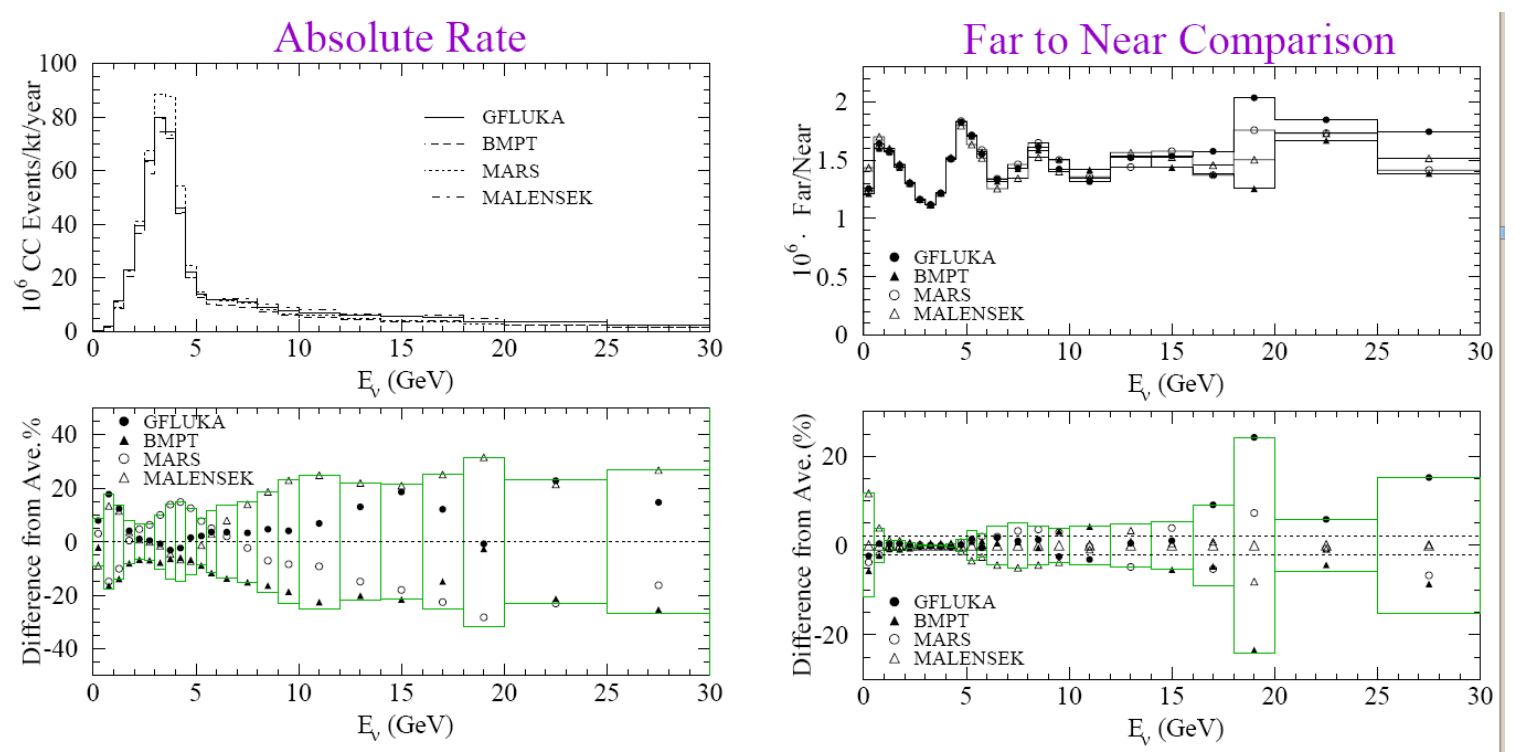

Figure 1.2: Uncertainties in neutrino flux calculation in NuMI beam. Since the MINOS near detector sees a line source of neutrino and far detector sees a point source, these uncertainties do not cancel for all momenta of interest, and having better understanding of hadronic production minimizes systematic error due to neutrino flux prediction.
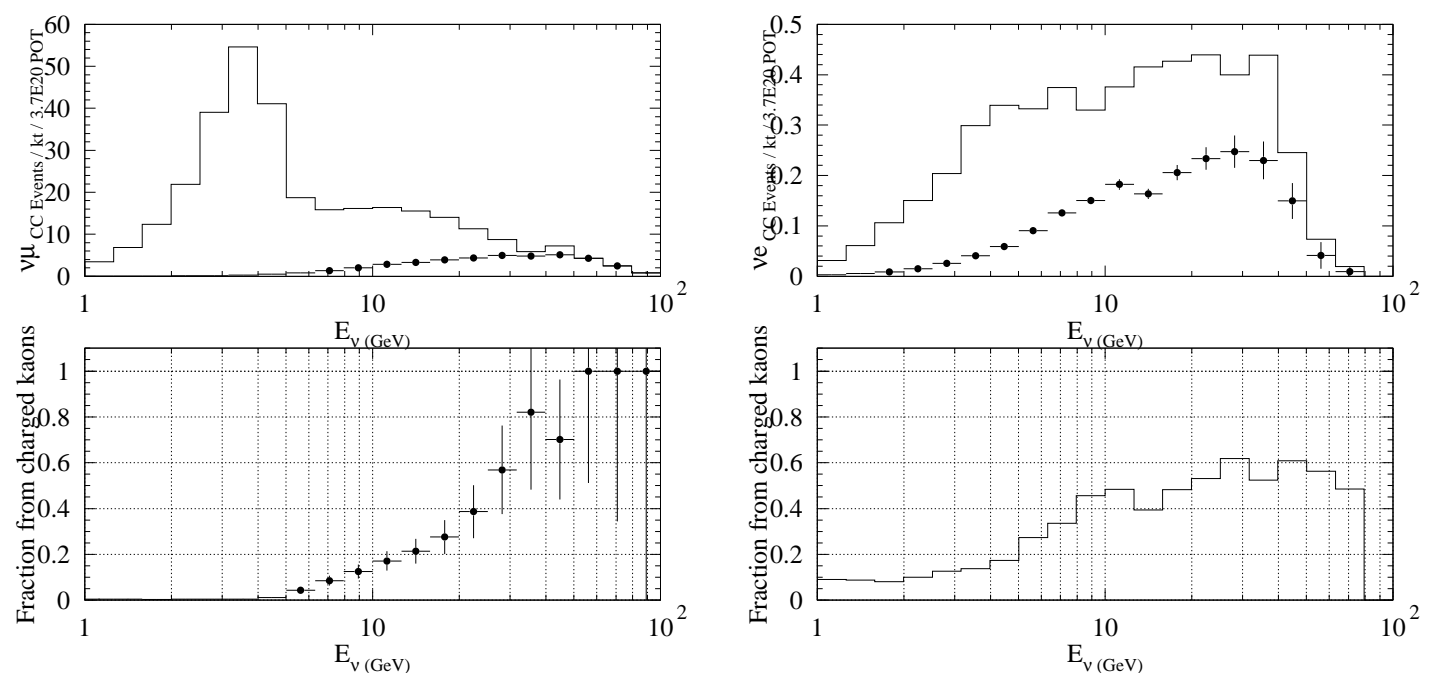

Figure 1.3: MINOS muon neutrino (left) and electron neutrino (right) fluxes and the fraction of neutrinos produced in kaon decays ${ }_{\boldsymbol{A}}$ 
at Fermilab. A time projection chamber (TPC) provides nearly complete coverage for the forward-going particles, and wire chambers allow measurement of momentum up to $120 \mathrm{GeV} / c$. Particle identification can be done for nearly all momenta of particles produced on the target using energy loss in the TPC, time of flight, threshold Cherenkov, and ring imaging Cherenkov counters.

In addition to taking data on thin nuclear target, MIPP experiment directly measured particle production on a replica of the NuMI target. Thus it is possible to cross check the understanding of NuMI hadron flux by comparing the spectrum from the actual target to the model of secondary production by using the proton carbon data at $120 \mathrm{GeV} / \mathrm{c}$ and hadron carbon data at lower momenta. This improved understanding of hadronic flux will be useful to the MINOS collaboration when in a few years statistical errors improve to their current systematic error from particle production.

Measurement of the pion/kaon production ratio $\Lambda^{\text {with }} p_{z}>20 \mathrm{GeV} / c$ presented here is the first step towards better understanding of NuMI hadron flux using MIPP data. 


\section{Chapter 2}

\section{MIPP Detectors}

The MIPP spectrometer, shown in Figure 2.1 consists of a number of subsystems designed to provide tracking and identification of charged particles. The experimental target is located within a few centimeters of the front of the TPC. The chamber allows to reconstruct tracks in three dimensions and measure momentum $\mathrm{m}_{\lambda}$ of low momentum tracks. Two magnets, Jolly Green Giant (JGG) and Rosie, have vertical magnetic fields in opposite directions. The net effect of the two magnets is a transverse displacement of the track from the straight line in the $x z$-plane. Position measurement in the four drift chambers (DCs) and two proportional wire chambers (PWCs) make it possible to measure track momentum of high-momentum tracks. Charged particle identification is done by different detectors TPC, time of flight wall (TOF), threshold Cherenkov (CKOV), and ring imaging Cherenkov (RICH) counters, depending on momentum of the particle. Finally, electromagnetic and hadron calorimeters make it possible to identify electrons, gamma rays, and neutral particles. All subsystems are described in detail below.

\section{$2.1 \quad$ Tracking}

The spectrometer provides data to reconstruct incoming beam particle and secondary particles from interactions on the target. Three small drift chambers upstream of the target (BCs) are able to reconstruct the incident particle trajectory (track). ${ }_{\Lambda}^{\mathrm{TPC}}$, four DCs, and two PWCs can reconstruct tracks coming out of an interaction. A summary of all wire chamber physical properties is given in Table 2.1 and operational conditions in Table 2.2. 


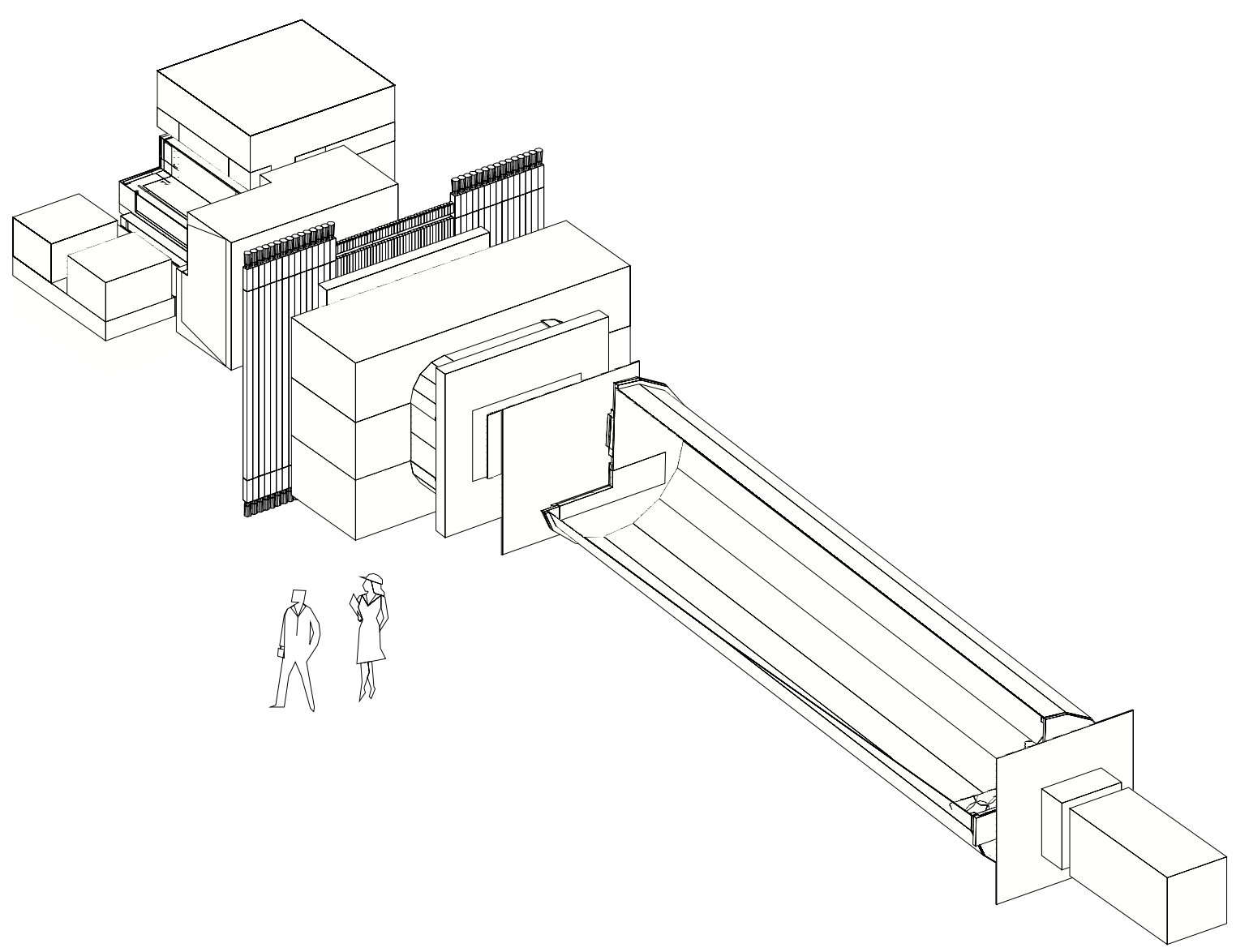

Figure 2.1: Schematic of the MIPP spectrometer. From upper left-hand corner shown are TPC inside the JGG magnet, threshold Cherenkov, time of flight wall, Rosie magnet, DC4, PWC5, RICH, PWC6, electromagnetic calorimeter, and hadron calorimeter. 


\begin{tabular}{|l|l|c|l|l|l|}
\hline \hline & $\begin{array}{l}\text { Wire } \\
\text { spacing } \\
(\mathrm{mm})\end{array}$ & Active area $(\mathrm{cm})$ & $\begin{array}{l}\text { Number } \\
\text { of wires }\end{array}$ & $\begin{array}{l}\text { Wire orientation } \\
\text { with respect to } \\
\text { vertical }\end{array}$ & $\begin{array}{l}z \text { in MIPP } \\
\text { geometry }(\mathrm{cm})\end{array}$ \\
\hline BC1 & 1.016 & $15.24 \times 10.16$ & 160 & $\pm 21.60^{\circ}, \pm 7.93^{\circ}$ & -4821.04 \\
$\mathrm{BC} 2$ & 1.016 & $15.24 \times 10.16$ & 160 & $\pm 21.60^{\circ}, \pm 7.93^{\circ}$ & -2465.86 \\
$\mathrm{BC} 3$ & 1.016 & $15.24 \times 10.16$ & 160 & $\pm 21.60^{\circ}, \pm 7.93^{\circ}$ & -1176.31 \\
$\mathrm{DC} 1$ & 3.4925 & $182.88 \times 121.92$ & 512 & $\pm 21.60^{\circ}, \pm 7.93^{\circ}$ & -586.83 \\
DC2 & 3.175 & $152.40 \times 101.60$ & $512(448)$ & $\pm 21.60^{\circ}, \pm 7.93^{\circ}$ & -427.99 \\
DC3 & 3.175 & $152.40 \times 101.60$ & $512(448)$ & $\pm 21.60^{\circ}, \pm 7.93^{\circ}$ & -352.62 \\
DC4 & 3.175 & $152.40 \times 101.60$ & $512(448)$ & $\pm 21.60^{\circ}, \pm 7.93^{\circ}$ & 63.38 \\
PWC5 & 3.000 & $200.00 \times 200.00$ & 640 & $0^{\circ}, 90^{\circ}, \pm 28.07^{\circ}$ & 132.99 \\
PWC6 & 3.000 & $200.00 \times 200.00$ & 640 & $0^{\circ}, 90^{\circ}, \pm 28.07^{\circ}$ & 1415.92 \\
\hline \hline
\end{tabular}

Table 2.1: Summary of multi-wire chambers parameters. DC2-4 have 448 wires in the wide-angle planes and 512 in narrow-angle planes. Position along the beam axis is given in offline geometry coordinates. $z=0$ is inside the Rosie volume, and the target is located at $z=-829 \mathrm{~cm}$.

\begin{tabular}{|l|c|c|}
\hline & Cathode voltage $(\mathrm{kV})$ & Gas \\
\hline Beam chambers & 1.4 & $82: 15: 3 \mathrm{Ar} / \mathrm{C}_{4} \mathrm{H}_{10} /$ methylal \\
Drift chambers & $1.85-2.05$ & $69: 28: 3 \mathrm{Ar} / \mathrm{C}_{4} \mathrm{H}_{10} /$ methylal \\
Proportional chambers & 3.0 & $76.5: 8.5: 15 \mathrm{Ar} / \mathrm{CH}_{4} / \mathrm{CF}_{4}$ \\
\hline
\end{tabular}

Table 2.2: Summary of chamber operation conditions 


\subsubsection{Drift Chambers}

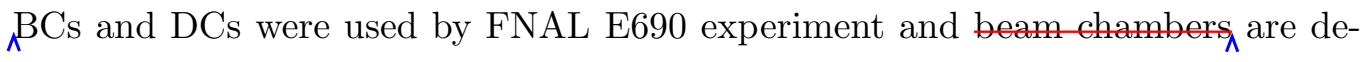
scribed in detail in [10]. Beam chambers span a distance of $37 \mathrm{~m}$ and thus provide $150 \mu \mathrm{m}$ resolution of position and $5 \mu \mathrm{rad}$ resolution of angle of the incoming particle when multiple scattering is negligible. Moreover, $1 \mathrm{~mm}$ wire spacing allows to distinguish multiple particles and $1 \mu \mathrm{s}$ range of the TDC allows one to measure the time of the track with respect to the trigger signal.

Chamber electronics consists of preamplifiers, Nevis discriminators, and $\mathrm{L}_{\Lambda}$ Croy 4290 CAMAC TDC system to measure drift time with 1 ns resolution. LeCroy 4290 system consists of 32-channel TDC CAMAC modules, organized in a total of 15 CAMAC crates with 20-22 modules per crate. Each crate was read out by a dedicated CAMAC crate controller. When triggered, controllers zero suppress the data and send them out to a module located in a normal CAMAC crate.

\subsubsection{Proportional Chambers}

The two PWCs straddling the the RICH counter were used in the NA24 experiment at CERN and later at the SELEX experiment at FNAL. Chamber construction is documented in [13].

These chambers are readput with RMH electronics[31] designed at CERN in the 1970's. Preamplified signals from all 8 planes $_{\bar{\lambda}}$ are discriminated and latched during $500 \mathrm{~ns}$ gate in 8 crates, each filled with 20 32-channel cards. Zero-suppressed readout consists of up to 4000 16-bit words indicating which channel of which module had a signal above threshold.

Unfortunately, only two of four planes in PWC5 could hold high voltage, which made the chamber less efficient and made it impossible to reduce combinatorial combinations of hit positions. However, these problems had a small effect on track reconstruction.

\subsubsection{Time Projection Chamber}

The TPC is the centerpiece of the MIPP spectrometer. With particle position measured at tens of points, the chamber is an excellent detector for pattern recognition of the entire event. It was constructed in 1990 at the Lawrence Berkeley Laboratory BEVALAC 

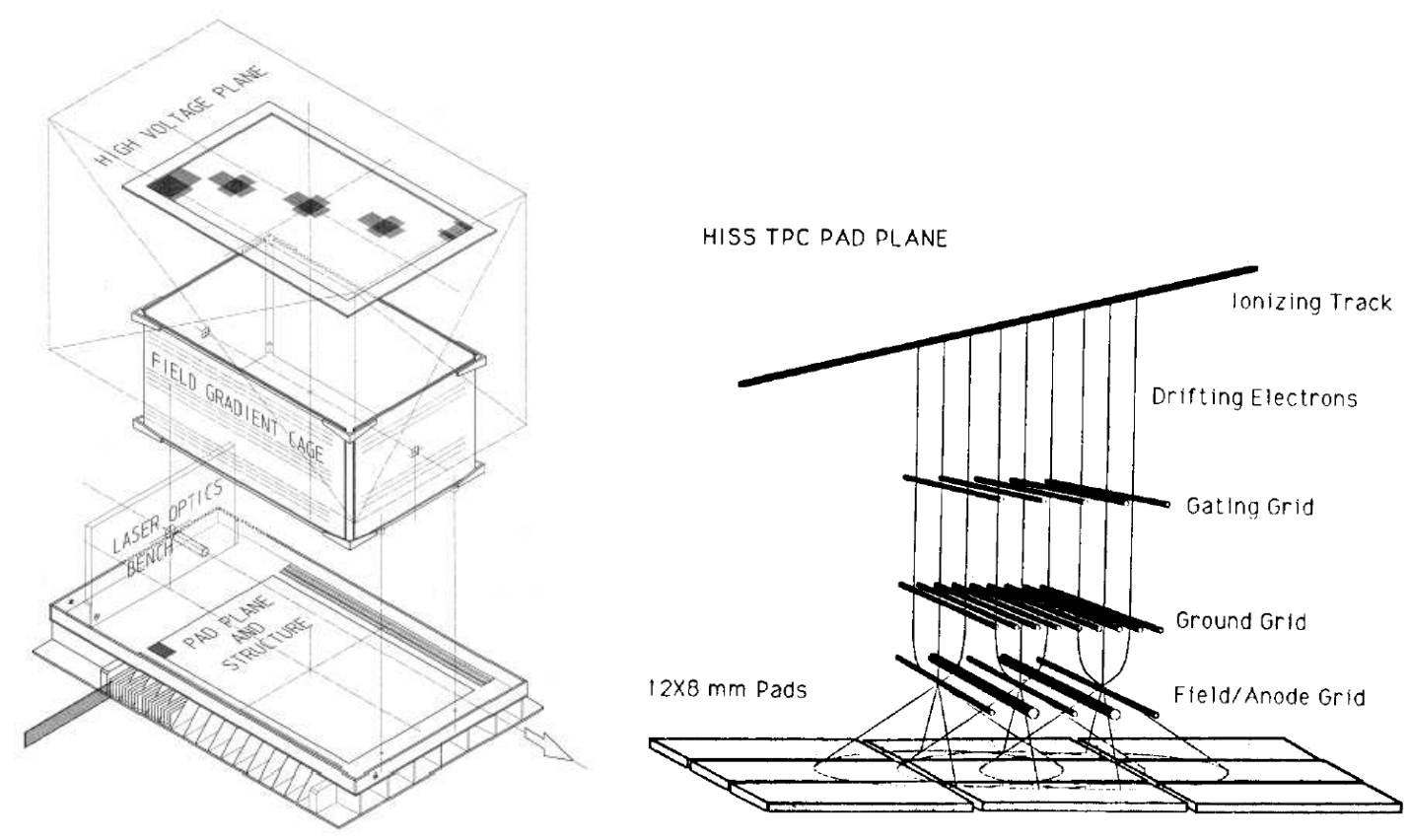

Figure 2.2: Schematic view of the TPC volume and pad plane with anode and ground wires, taken from [17].

facility for EOS experiment[4, 22, 17, 27, 35, 42, 18]. Prior to being used by the MIPP experiment, it served ${ }_{\wedge}$ BNL E895 and E910 experiments.

Electrons ionized in the $96 \times 162 \times 81 \mathrm{~cm}^{3}$ gas volume by charged particles drift down in uniform vertical electric field of $125 \mathrm{~V} / \mathrm{cm}$. At the bottom of the drift volume (see Figure 2.2), in the region between ground and anode wires, an avalanche is created by

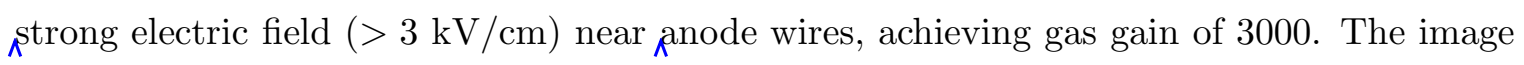
charge on the pad plane $4 \mathrm{~mm}$ below the anode wires is recorded by the electronics.

The pad plane is divided into 128 paddrows along the $z$-axis with $1200.8 \times 1.2 \mathrm{~cm}$ pads per row along the $x$-axis. Padrows record a slice of ionization energy in $z$. Each pad is digitized by 12-bit flash ADC at $10 \mathrm{MHz}$. With drift velocity of $\approx 5 \mathrm{~cm} / \mu \mathrm{s}$, this gives $5 \mathrm{~mm}$ sampling along the vertical. A typical minimum ionizing track would leave signal on about 3 pads in a given row, with amplified signal spread over seven $100 \mathrm{~ns}$ time buckets. The large number of measurement points (up to 128 per track), yields sub-millimeter position resolution on the track position.

The TPC is readout through dedicated VME boards organized into four crates. Fiber optics links connect ${ }_{\Lambda} \mathrm{VME}$ boards to the pre-amplifier/shaper "sticks" located under- 
neath the pad plane. A stick covers two half padrows.

\subsection{Particle Identification}

The spectrometer allows to identify the incident particle and secondary particles. Incident particles are identified using two differential Cherenkov counters: one for $\pi / \mathrm{K}$ and one for $\mathrm{K} / \mathrm{p}$ separation. Secondary particles are identified by different detectors depending on their momentum. From low to high momentum the detectors are the TPC $(<1 \mathrm{GeV} / c$ ), the TOF wall $(<3 \mathrm{GeV} / c)$, the CKOV $(<17 \mathrm{GeV} / c)$, and the RICH for particles with higher momenta.

\subsubsection{Beam Cherenkov}

Each beam Cherenkov counter consists of a long cylindrical radiator volume and a head at the downstream end.-The head contains a focusing mirror which reflects the light onto a photomultiplier tube (PMT) covered by a second mirror with a hole. The aperture of the hole is chosen in such a way that Cherenkov light emitted at angles below the cutoff would hit the PMT behind the second mirror (inner PMT) whereas light emitted at larger angles is reflected onto the outer PMT. The cutoff Cherenkov angles for the inner PMTs are 5 and 7 mrad respectively for the upstream and downstream counters. Radiator volume lengths are $22.9 \mathrm{~m}$ and $12.2 \mathrm{~m}$ so that statistically the same number of photo-electrons hit inner PMT's on both counters.

Density in the upstream counter is set to achieve the kaon Cherenkov angle of $5 \mathrm{mrad}$ - focusing its light onto the inner PMT. Pions with identical momentum radiate light at a larger angle, missing the inner PMT and hitting the outer PMT. At the momenta of interest $\left(\frac{\mathrm{Q} 0 \mathrm{GeV}}{\mathrm{h}} / \mathrm{c}\right)$, protons do not radiate at all under these conditions.

Similarly, the pressure in the downstream counter is set so that the proton Cherenkov light hits the inner PMT, but not the outer PMT. The outer PMT collects light emitted at angles less than $30 \mathrm{mrad}$. This is important since for momenta below $\approx 32 \mathrm{GeV} / c$ the pion Cherenkov angle is so large that the photons emitted by pions are no longer collected on the outer PMT. This property is independent of the gas that fills the radiator volume.

One of the challenges for identification of incident particle is the large span of momenta (5-90 GeV/c). The counters are tuned to different beam momentum by establishing

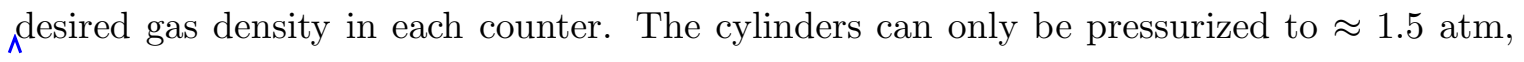


therefore at low momenta a heavy non-flammable gas has to be used in order to obtain a positive tag for protons below $30 \mathrm{GeV} / c$ and for kaons below $20 \mathrm{GeV} / c$. MIPP used $\mathrm{C}_{4} \mathrm{~F}_{8} \mathrm{O}$ for this purpose. Nitrogen at different densities was used for all higher momenta. However, at $5 \mathrm{GeV} / c$ even the heavy gas is not dense enough for kaons or protons to radiate. But at that momentum we can take advantage of the measurable difference in time of flight of the three particle species between the front of the beamline and experimental target.

\subsubsection{Threshold Cherenkov}

The threshold Cherenkov consists of 96 toroidal mirrors at the downstream wall of the counter filled with heavy gas. Each mirror is positioned to focus Cherenkov light onto one of the 96 PMT's positioned at the top and bottom of the counter. The mirrors are positioned in such a way that each mirror reflects Cherenkov light onto one and only one PMT if the particle emanates from the target. In some instances, of course, light is shared by 2 or 3 mirrors, which can be taken into account once track trajectory is known. ${ }_{\wedge}^{\mathrm{PMT}}$ windows are coated with wavelength shifter to make them sensitive to ultraviolet light. ${ }_{\Lambda}$ Charge off the PMTs is digitized with LeCroy 4300 ADC modules and discriminated signals are recorded with LeCroy 3377 multi-hit TDCs.

Historically, the CKOV was operated with Freon, but due to modern environmental regulations, we had to use a much more expensive alternative $\mathrm{C}_{4} \mathrm{~F}_{10}$. At atmospheric pressure, this gas has pion threshold at $2.6 \mathrm{GeV} / c$, kaon threshold at $8 \mathrm{GeV} / c$ and proton threshold at $17 \mathrm{GeV} / c$.

\subsubsection{Beam Time of Flight}

Three scintillator counters are placed in the beamline: T01 about $3 \mathrm{~m}$ upstream of the target, TBD about $20 \mathrm{~cm}$ upstream of BC1, and T00 another $20 \mathrm{~m}$ upstream, almost immediately after the secondary beamline collimator. These detectors had to satisfy the following objectives:

1. Form a beam trigger signal,

2. Provide a reference start time for the TOF wall,

3. Resolve pileup,

4. Identify incoming beam particles at momentum of $5 \mathrm{GeV} / c$. 


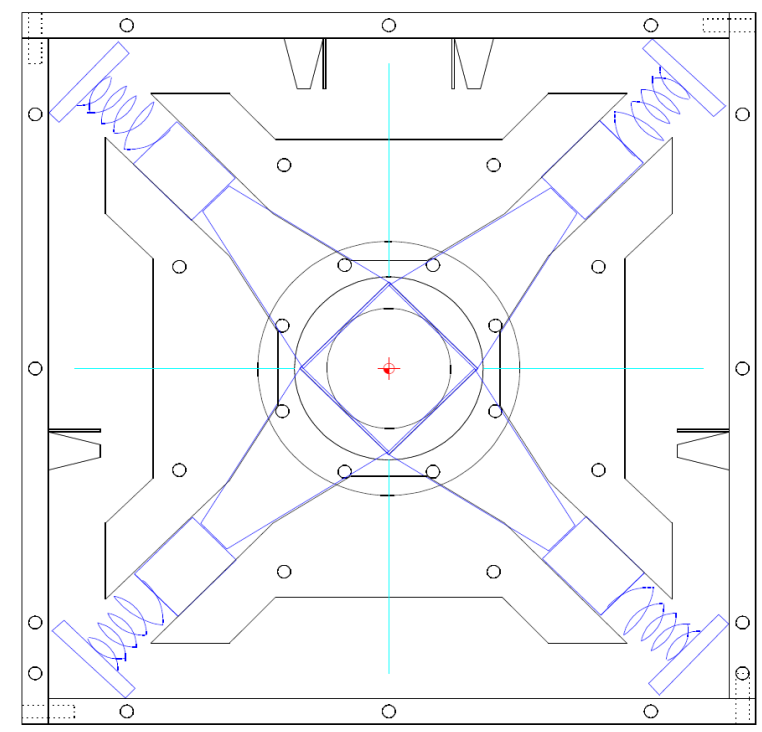

Figure 2.3: Schematic of T00 and T01 construction. Light from the scintillator is collected onto four PMT's by light guides. Phototubes are held in place by springs. TBD assembly is similar, except no light guides are used.

Each counter consists of a square piece of scintillator and four Hamamatsu R5900U PMT's, one on each side of the scintillator. Figure 2.3 shows schematic of the inside of the counters. The initial two counters T00 and T01 are equipped with light guides, which improve timing resolution. TBD counter, which was added at a later time to help form beam trigger does not have the light guides, as we were not planning on using it to measure time of flight of beam particles. In practice, degradation of timing resolution is dominated by delay cables, so resolution of TBD is comparable to ${ }_{\wedge}$ T01 counter, and exceeds that of T00 counter. Resolution of ${ }_{\Lambda}$ TBD-T01 time difference for $120 \mathrm{GeV} / c$ protons is $120 \mathrm{ps} \sigma$.

Each PMT signal is split with $90 \%$ of the charge to a discriminator and TDC, and $10 \%$ to an ADC. Knowing the amount of charge on a given PMT allows for offline hit time correction.

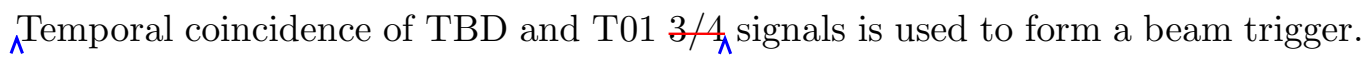
Further discussion of the experimental trigger is presented in Chapter 3.

Two sets of CAMAC TDCs measure PMT hit times with respect to experimental trigger time:

- LeCroy 3377 with 0.5 ns resolution and up to 16 hits in 512 ns window, 
- LeCroy 2229 with 30 ns resolution, but only 1 hit in 60 ns window.

The first set of TDCs helps to determine presence of pileup (multiple incident particles in one event), while the second set of TDCs is used to reconstruct particle flight time and serves as the reference time for the time of flight wall.

\subsubsection{Time of Flight Wall}

Conceptually the TOF wall is straightforward: $543-\mathrm{m}$ long vertical scintillator bars form a solid wall $3.5 \mathrm{~m}$ wide. The central $2 \mathrm{~m}$ are covered by $405 \times 5 \mathrm{~cm}$ bars and the wings are covered by $10 \times 10 \mathrm{~cm}$ bars. Both ends of each bar are capped with Winston cones to collect light onto Hamamatsu R5900U PMT's (identical tubes used in the beam counters).

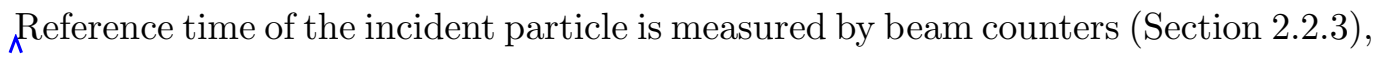
and time of arrival of resulting particles is measured on both ends of each scintillator bar. The segmentation of the wall is such that the probability of two particles hitting the same bar is small.

\subsubsection{Ring Imaging Cherenkov}

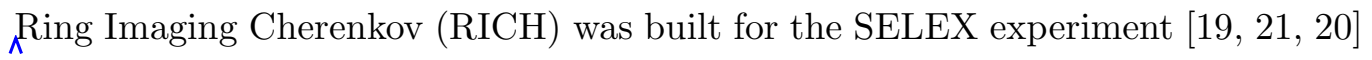
in the 1990's. We filled the $10 \mathrm{~m}$ long radiator volume with carbon dioxide to a total pressure of $\approx 1.03 \mathrm{~atm}$. In the course of the run, the volume was slowly leaking, so $\mathrm{CO}_{2}$ was added on a weekly basis keeping the pressure constant within $1 \%$. The level of oxygen contamination in the vessel was less than $1000 \mathrm{ppm}$.

Sixteen hexagonal spherical focusing mirrors mounted at the downstream end reflect Cherenkov light onto an array of 1 inch PMTs located on the upstream flange of the cylinder above the beam window. The mirrors cover an area of approximately $2 \times 1 \mathrm{~m}$. The average focal radius of mirrors is $19.8 \mathrm{~m}$ with RMS spread of less than $3 \mathrm{~cm}$. Prior to the run, mirrors were aligned with laser so that their foci were at the center of the PMT array.

A holder plate supports the PMT's in a hexagonally close-packed array of $89 \times 32$ tubes. The plate separates the array from radiator volume with 2848 individually glued quartz windows and holds aluminized mylar cones for each phototube $e_{\bar{\lambda}}$ ssentially $100 \%$ of reflected Cherenkov light that reaches the front of the vessel gets to the phototubes.

Light is detected with two different types of phototubes: 


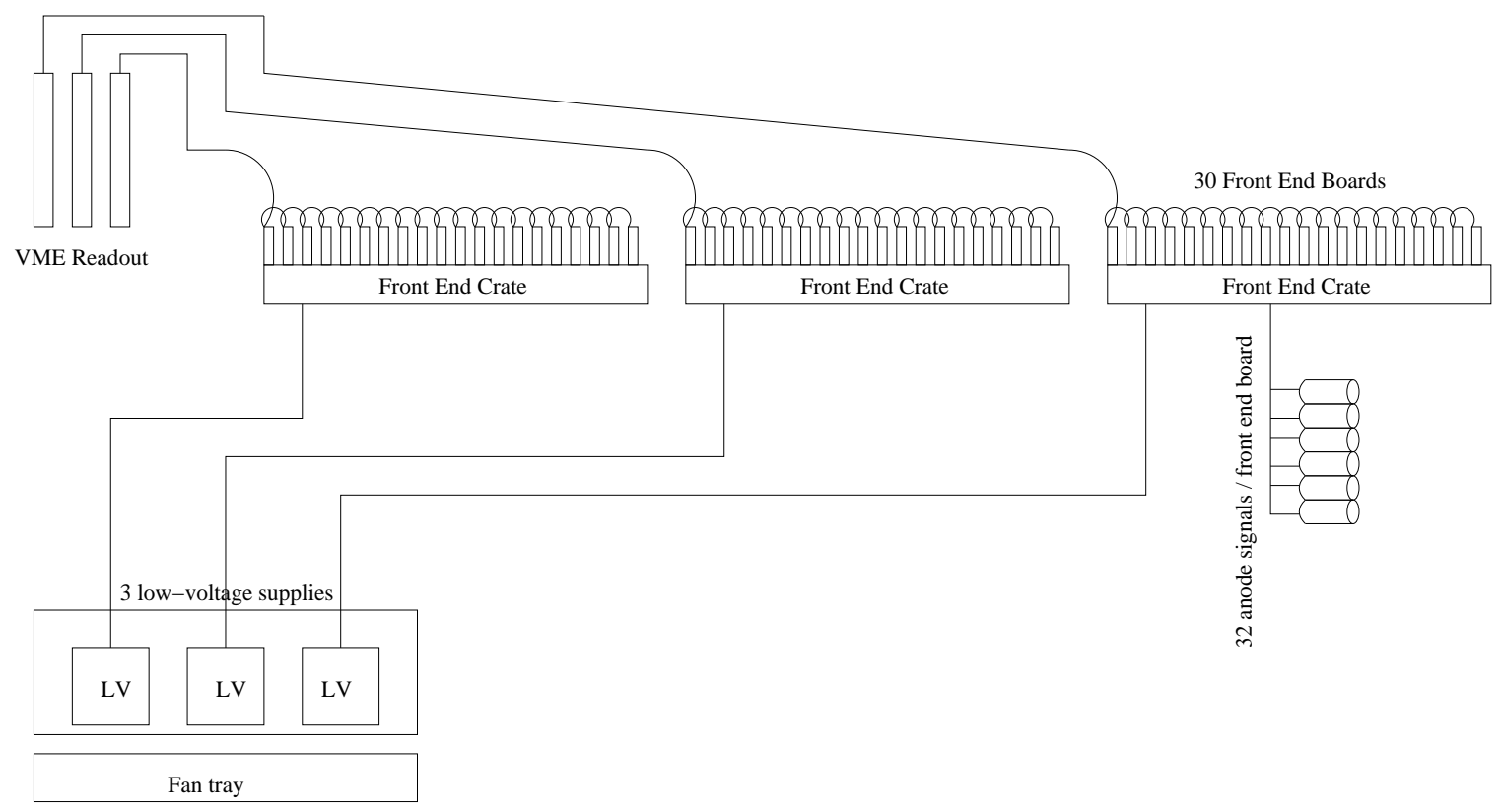

Figure 2.4: Schematic of RICH electronics setup.

- Hamamatsu R-760 with quartz windows and response down to $170 \mathrm{~nm}$.

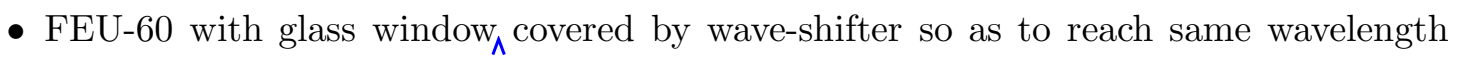
range as ${ }_{\Lambda}$ Hamamatsinu. These PMTs are about $42 \%$ as efficient, but they are 10 times as cheap.

Unfortunately, in March 2004 one of the FEU-60 PMT bases caught fire, resulting in $30 \%$ loss in the number of PMT's. Fortunately, this accident had a negligible impact on the PID capability of the device because of the high index of refraction of $\mathrm{CO}_{2}-$ radius of $\beta=1$ ring is $29.5 \mathrm{~cm}$ and typical number of hit tubes per ring is over 20 .

All photomultiplier tubes are powered with 6 high voltage supplies, each capable of delivering $200 \mathrm{~mA}$ at $3 \mathrm{kV}$. 32 PMT's with similar nominal high voltage were grouped together, and a number of different voltages in steps of $20 \mathrm{~V} \wedge$ created with 6 Zener diode chains.

The signal is read out with custom electronics designed at FNAL, built and tested at Harvard University. The setup is shown schematically in Figure 2.4. 89 front-end cards are arranged into 3 crates (30, 30, and 29 cards), each crate controlled by one VME controller board. 
Each of the 32 channels on front-end cards contains a differential amplifier with a gain of $\approx 20$ and an analog comparator. The threshold is programmable for each channel in steps of $0.8 \mathrm{mV}$. Discriminated signal is continuously pushed into a pipeline memory. When a controller board is triggered, the trigger is forwarded to front-end cards and the data that falls within the strobe gate is latched. RICH electronics can be strobed multiple times before a readout command must be issued. Pipeline delay is programmable for each front-end board and can be set as large as 128 clock ticks (2.4 $\mu$ s with $53 \mathrm{MHz}$ clock).

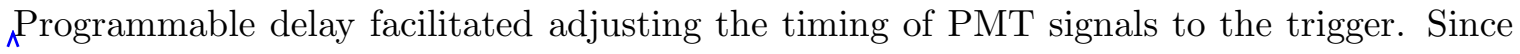
the instantaneous rate at MIPP was quite low, we latched the signal with a fairly wide strobe gate of $100 \mathrm{~ns}$. The electronics is capable of using gates as short as two clock cycles.

\subsubsection{Electromagnetic Calorimeter}

The primary purpose of electromagnetic calorimeter is to measure the production of forward high energy photons. It consists of 10 planes of alternating horizontal and vertical wire planes sandwiched between $15 \mathrm{~mm}$ thick lead plates. Each active plane is comprised of eight 8 inch ${ }_{\lambda}$ wide, 1 inch ${ }_{\lambda}$ thick chambers with 8 wires per chamber. Front view of the system is shown in Figure 2.5.

${ }_{\Lambda}$ Detector iss readout with custom ADC CAMAC units built by the University of Michigan group. Because 1970's ADC chips are used, the calorimeter is the second slowest detector after TPC in terms of readout speed.

\subsubsection{Hadron Calorimeter}

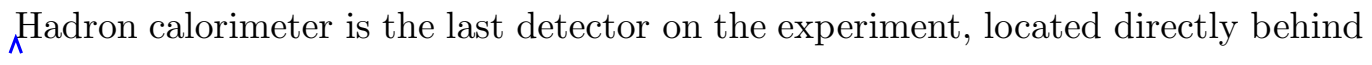

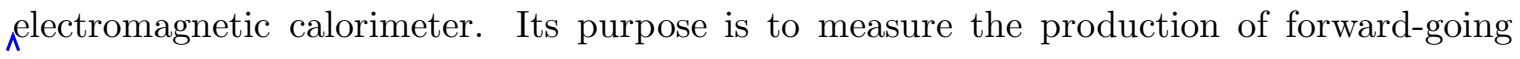
neutrons. It was previously used by the HyperCP[7] experiment. The active volume consists of four scintillator plates sandwiched between lead sheets. It is meant to determine the energy of hadrons by summing $\Lambda_{\Lambda}$ energy deposition of the resulting hadronic shower initiated in the lead sheets that comprise the majority of the interaction lengths of the detector.

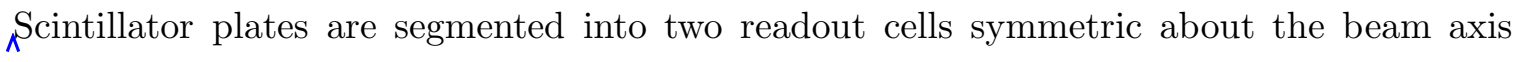
(with a total of eight cells). Each cell is read out by one photomultiplier tube. The calorimeter also serves as the experiment's beam dump

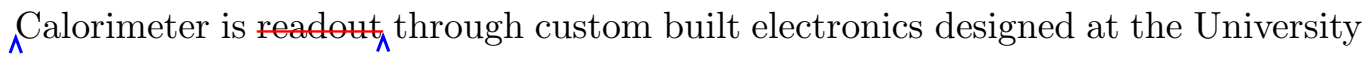




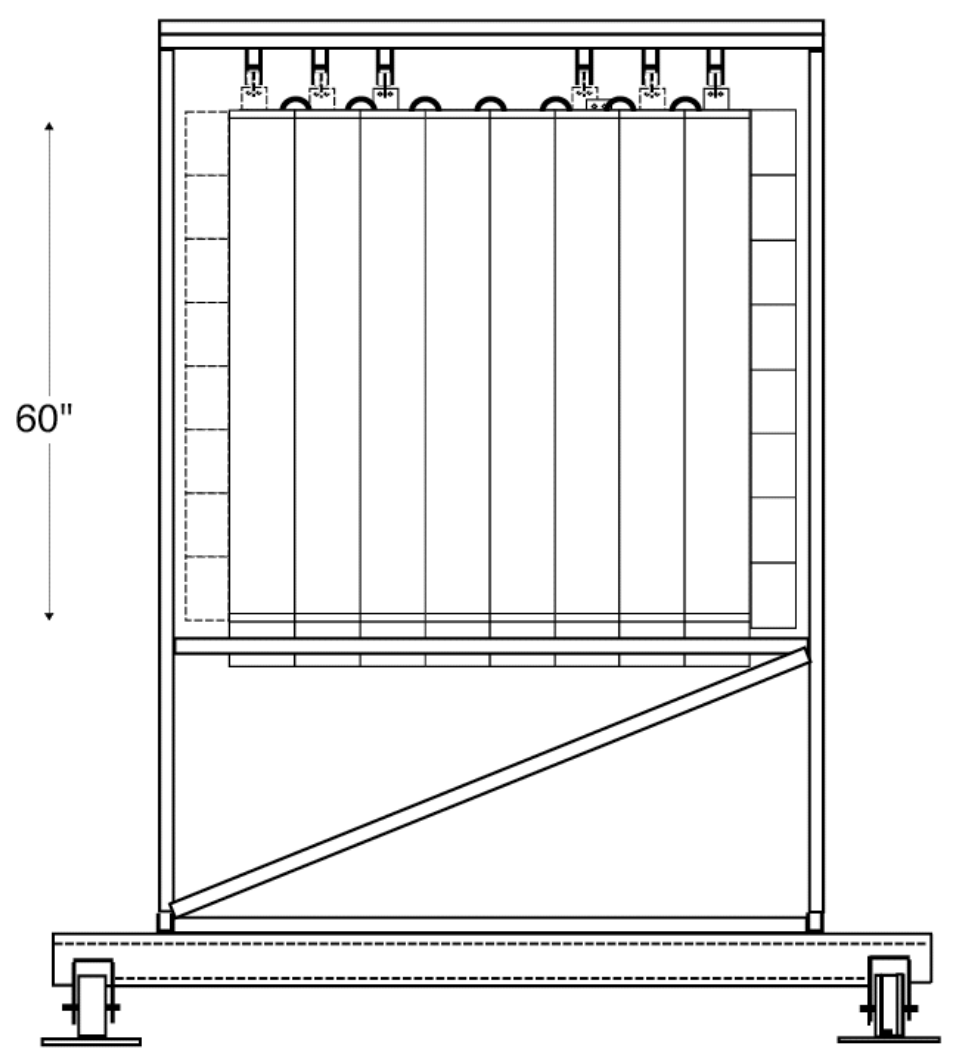

Figure 2.5: Electromagnetic calorimeter front view. 


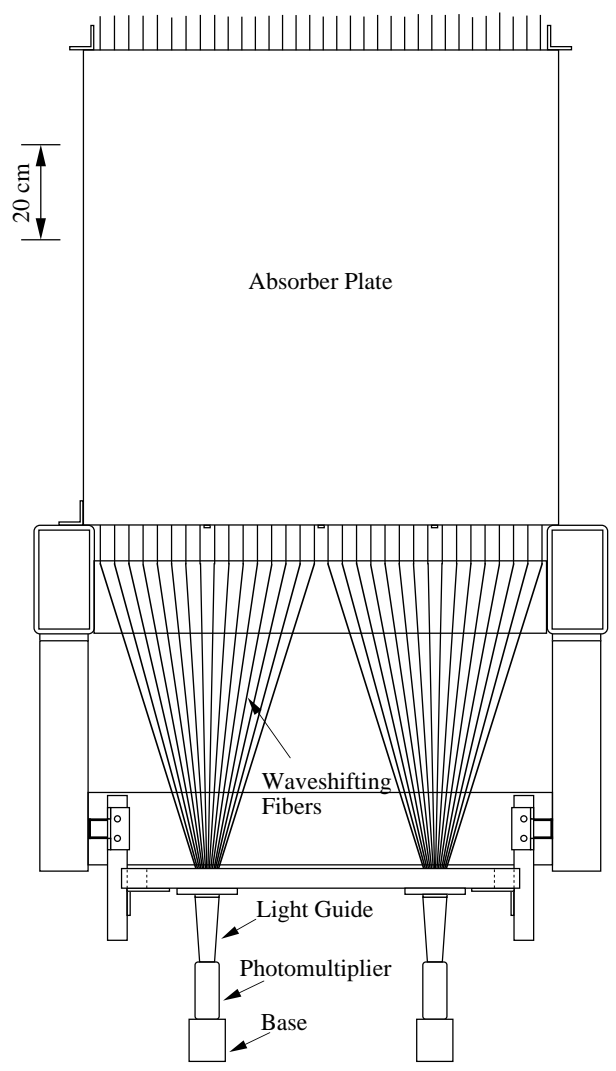

Figure 2.6: Schematic of hadron calorimeter front view. One can see the two cells, the light from each cell is collected onto its respective phototube. 

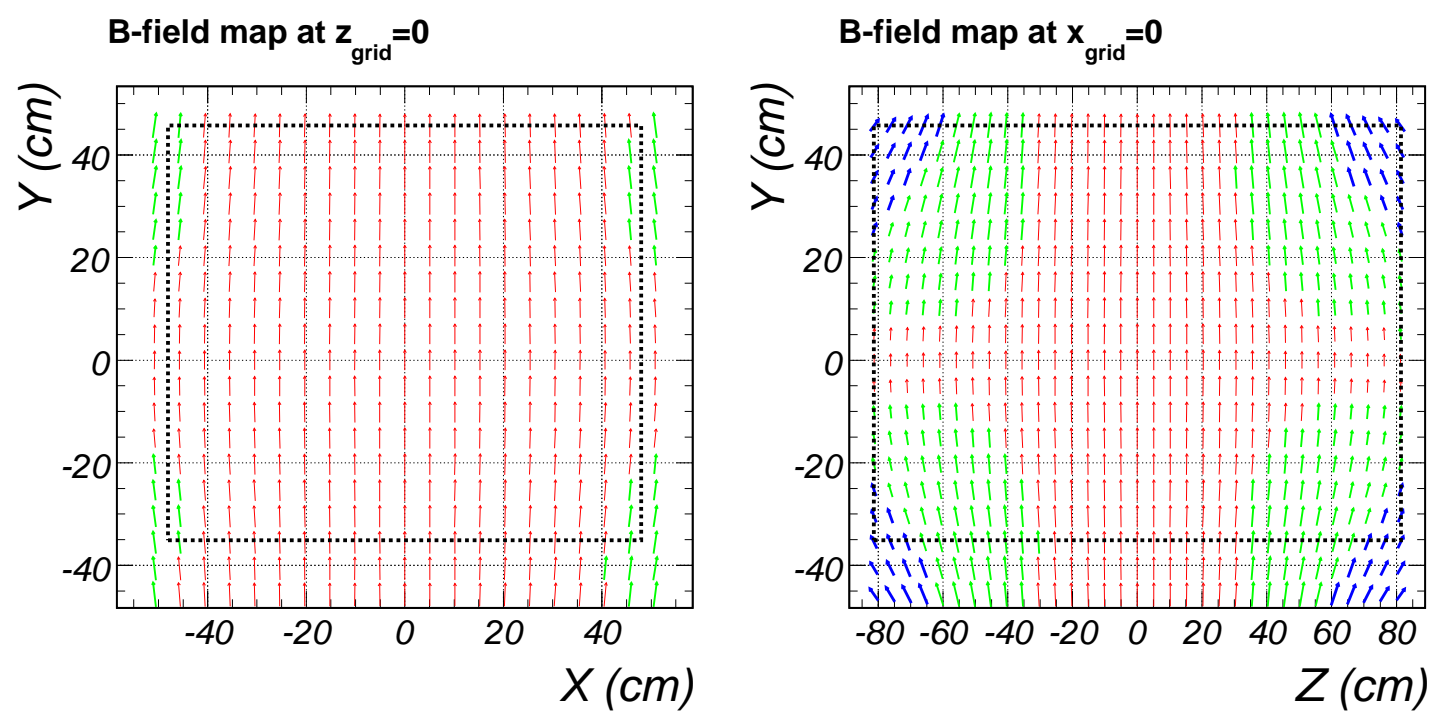

Figure 2.7: Projections of the JGG field map in slices along the center of the magnet. Regions of non-uniform field are highlighted with thicker arrows where $B_{i} / B_{y}>0.1$ and thickest arrows where $B_{i} / B_{y}>0.5$. The dashed line shows the boundary of the TPC drift volume.

of Virginia. ${ }_{\Lambda}^{\mathrm{PMT}}$ signal passes through ${ }_{\wedge}^{\mathrm{ADC}}$ driver module and the amplified charge is then digitized by a 16-bit CAMAC ADC. With pedestal widths of $2-3$ ADC counts, each cell has dynamic range of about 5000 .

\subsection{Analysis Magnets}

MIPP uses two magnets for momentum measurement: Jolly Green Giant and Rosie. For safety, magnetic fields are set so that the kick angles are about equal and opposite $\left(\int B_{y} d l \approx 1 \mathrm{Tm}\right)$. This way regardless of momentum, beam is absorbed by the beam dump.

While the experimental hall was still empty, all three magnet field components were mapped onto $\mathbb{R}^{2}$-inch three dimensional grid using Ziptrack field measuring device. The device consists of three Hall probes, one for each component of the magnetic field. As the probe holder glides along the track, the field is sampled every 2 inches, with the three components sampled in the same point at slightly different times. Complete field maps are used to compute trajectories of particles in the non-uniform field and to compute trajectory of electron drift in the TPC. 
Rosie field map projection

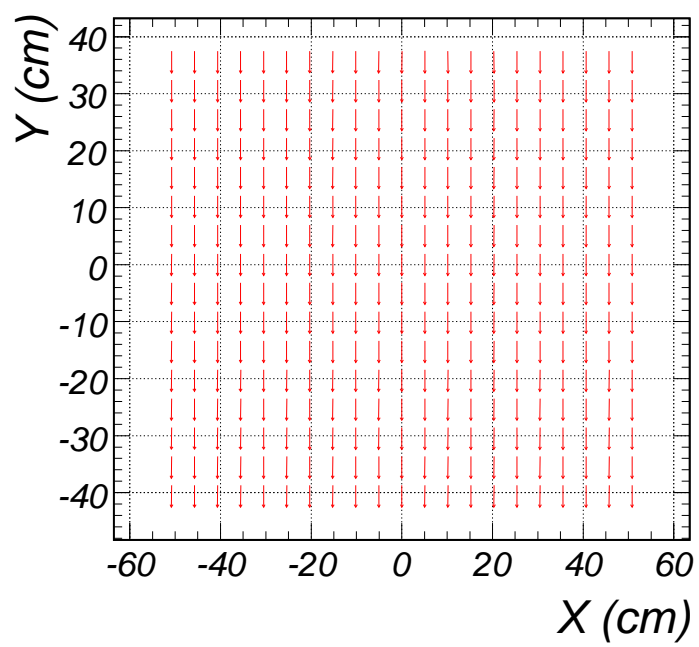

Rosie field map projection

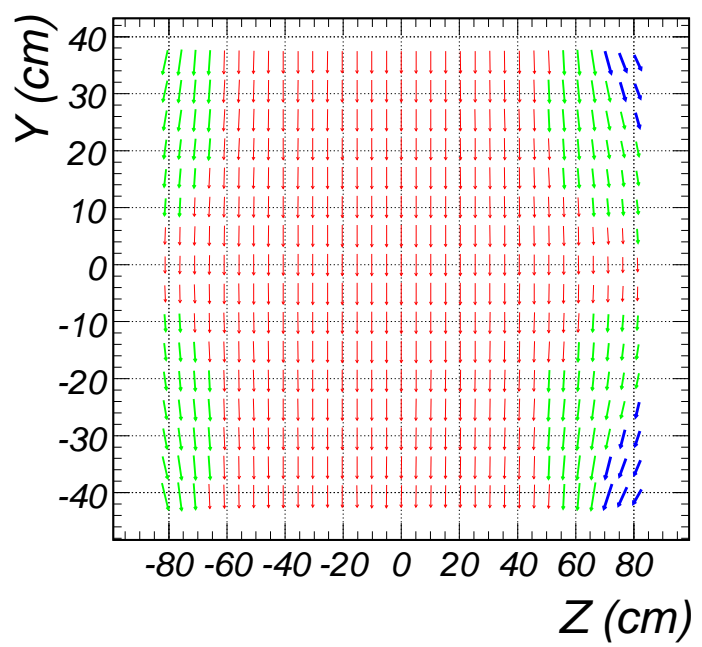

Figure 2.8: Projections of Rosie field map in the same volume as those in Figure 2.7, using the same color scheme for arrows.

The Jolly Green Giant magnet was originally built at Harvard in the 1960's. Its aperture is sufficiently large for the TPC to fit, but its magnetic field is non-uniform towards the edges of the TPC drift volume, hence corrections of up to $7 \mathrm{~cm}$ in TPC hit positions have to be made. Rosie magnetic field is much more uniform, but unfortunately, its aperture is a few centimeters too small for the TPC to fit.

One of the coils in Jolly Green Giant was damaged and had been repaired prior to magnet being installed in the MIPP hall. In the course of the run, two coils shorted out, so the experiment ended up having 3 distinct field maps. Only the first map was measured with Ziptrack, but computer models of the magnetic field showed that the differences in field components due to different coil combination are small and can be neglected. 


\section{Chapter 3}

\section{MIPP Trigger}

${ }_{\Lambda}$ Experimental single-level trigger is fairly simple: the first stage of the trigger decides on the species of the particle and the second stage on whether or not the particle interacted in the target. Thus, 7 physics trigger bits are typically in use: untagged beam, three particle species, and three particle species with interaction. Different trigger bits are scaled down before a global OR is formed in order to achieve about $20 \%$ minimum bias and $80 \%$ interaction trigger mix. Unless prohibitively high scale dow factors are required, the factors are chosen to record an even mix of protons, kaons, and pions.

Since the trigger has only one level and does not provide a fast clear, decision has to be made in the shortest time possible so as not to degrade the resolution of the TOF system. In the final implementation, the trigger is formed about $250 \mathrm{~ns}$ after the incident particle passes through the target.

\subsection{Particle Identification}

\subsubsection{Momentum Above $20 \mathrm{GeV} / c$}

As described in Section 2.2.1, at $20 \mathrm{GeV} / c$ and above, the pressure in beam Cherenkov counters can be tuned so that all charged particles radiate light. The four PMTs are abbreviated as $U I$ for upstream inner, $U O$ for upstream outer, $D I$ for downstream inner, and $D O$ for downstream outer. Under these operating conditions, the particle trigger bits are defined as:

- $\pi: \overline{U I} \cdot U O$ 
- $\mathrm{K}: U I \cdot \overline{U O}$

- p: $D I \cdot \overline{D O}$

At $35 \mathrm{GeV} / c$ and above, pion and kaon Cherenkov angle in the downstream counter is below $30 \mathrm{mrad}$, so under those conditions, a more stringent trigger could be formed, based on all four PMTs:

- $\pi: \overline{U I} \cdot U O \cdot \overline{D I} \cdot D O$

- $\mathrm{K}: U I \cdot \overline{U O} \cdot \overline{D I} \cdot D O$

- p: $\overline{U I} \cdot \overline{U O} \cdot D I \cdot \overline{D O}$

In the interest of saving trigger time, this momentum-dependent trigger was not implemented.

\subsubsection{5 $5 \mathrm{GeV} / c$ Momentum}

Using beam Cherenkov counters, it is not possible to tag protons with momentum below about $20 \mathrm{GeV} / c$, and kaons cannot be tagged when momentum is below $11 \mathrm{GeV} / c$, as a sufficiently high gas density could not be obtained. Even if it were possible to do that, the gas density would be so large that multiple scattering in the counters would degrade the knowledge of position and direction of the incoming particle. Instead, at $5 \mathrm{GeV} / c$, we rely on particle time of flight to distinguish kaons and protons offline. Figure 3.1 shows that the task is easily achieved at such a low momentum.

For the purpose of the trigger, both beam Cherenkov densities were tuned to radiate on pions, muons, and electrons. Then proton plus kaon trigger was the absence of signal in all beam Cherenkov PMTs. Both negative and positive beams of this momentum are primarily $(\approx 90 \%)$ composed of pions, with similar fractions of proton $n_{\lambda}$ and kaon $_{\mathcal{N}}$ so it was acceptable to accumulate equal fractions of protons and kaons, as long as they could be distinguished offline.

\subsection{Beam Cherenkov Operation}

Pressure scans were done in order to find the optimal operating gas densities of the beam Cherenkov counters. A stand-alone executable read out latched discriminated 


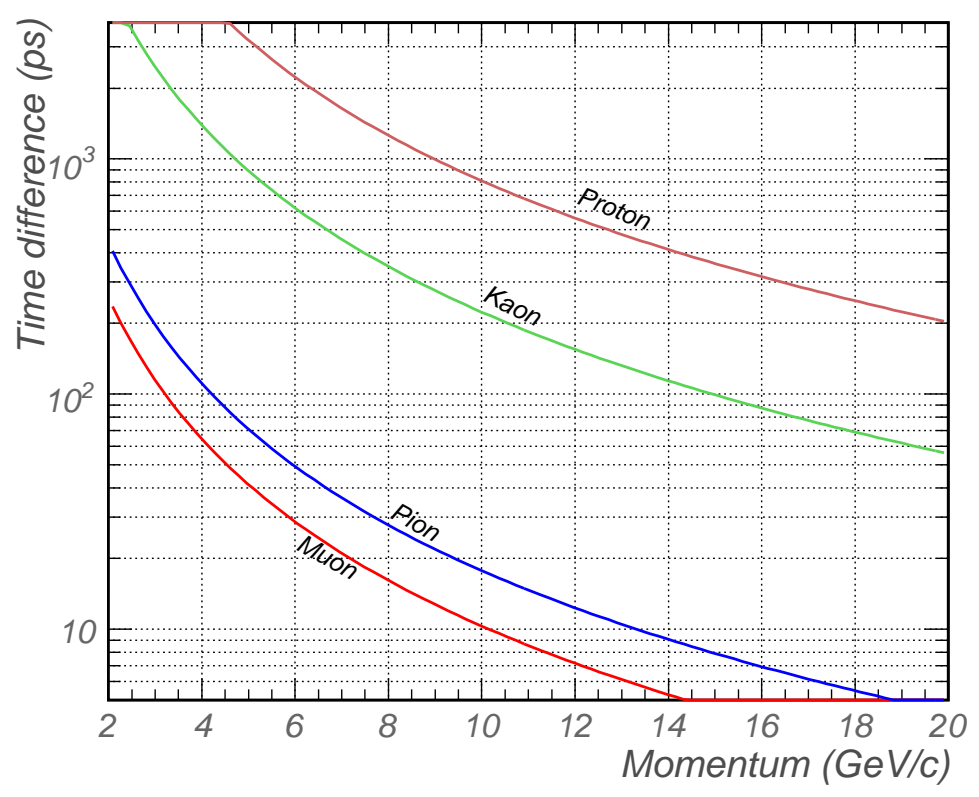

Figure 3.1: Time of flight difference from $\beta-1$ particle for muons, pions, kaons, and protons between T00 and T01 counters. With time resolution of $200 \mathrm{ps}$, it is possible to do proton/kaon separation below $11 \mathrm{GeV} / c$, and kaon/pion separation below $6 \mathrm{GeV} / c$. 


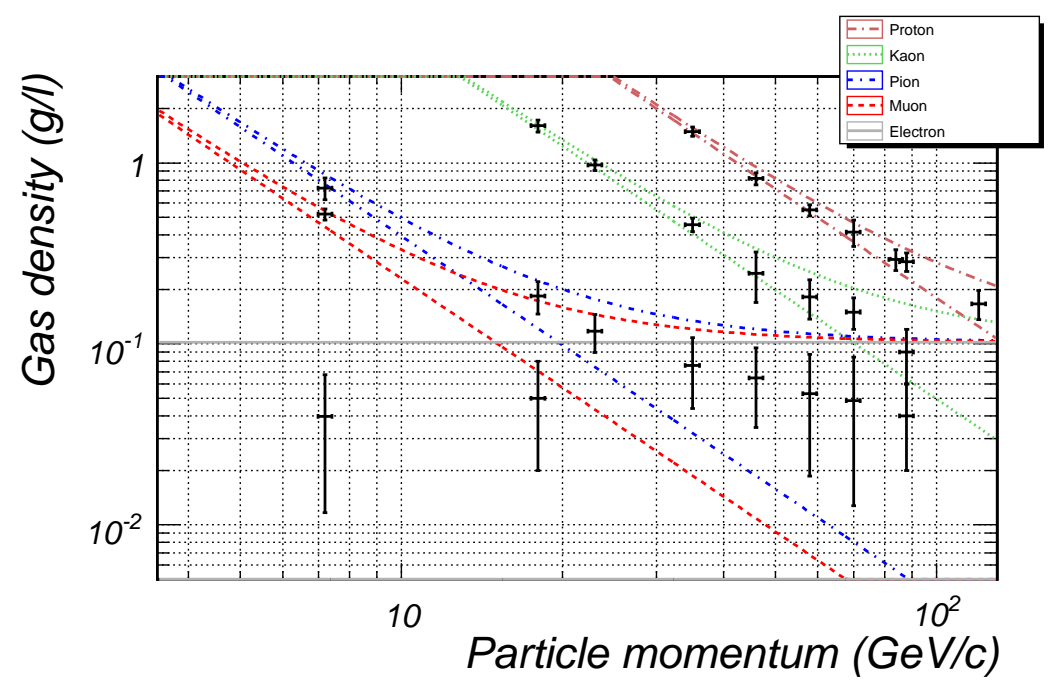

Figure 3.2: Summary of upstream beam Cherenkov pressure curves with nitrogen. The two curves for each particle species are ${ }_{\Lambda}$ threshold density and density at which ${ }_{\Lambda}$ Cherenkov angle is $5 \mathrm{mrad}$. Superimposed data are $\hat{\Lambda}^{\mathrm{s}}$ enters of peaks of $U I \cdot \overline{U O}$ in pressure curves.

PMT signals at different pressures, recording 10000 triggers per pressure point. From the frequency of occurrence of the 16 different PMT combinations, one can find the peaks corresponding to the different particle species. Figure 3.2 shows the summary of all pressure scans data in nitrogen.

For momenta $20 \mathrm{GeV} / c$ and above, RICH rings can be used to measure $\Lambda^{\text {trigger }}$ purity. In $20 \mathrm{GeV} / c$ beam, we can identify protons by lack of signal, but at $35 \mathrm{GeV} / c$ and above, all 3 particles radiate, so purity measurements shown in Figure 3.3 are more reliable. Still, in most cases, trigger purity is above $70 \%$, and as one would expect, it is above $99 \%$ for majority particle.

\subsection{Interaction Trigger}

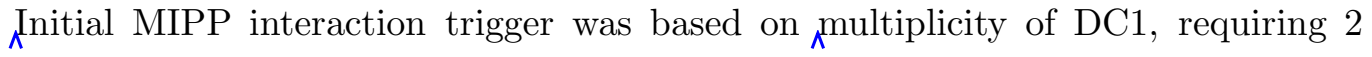
or more wire clusters in at least three of the four planes. This trigger has the following problems associated with it:

a) Large chamber area made it highly susceptible to poor beam conditions,

b) ${ }_{\wedge}$ Chamber is sometimes noisy because of low discriminator thresholds, 


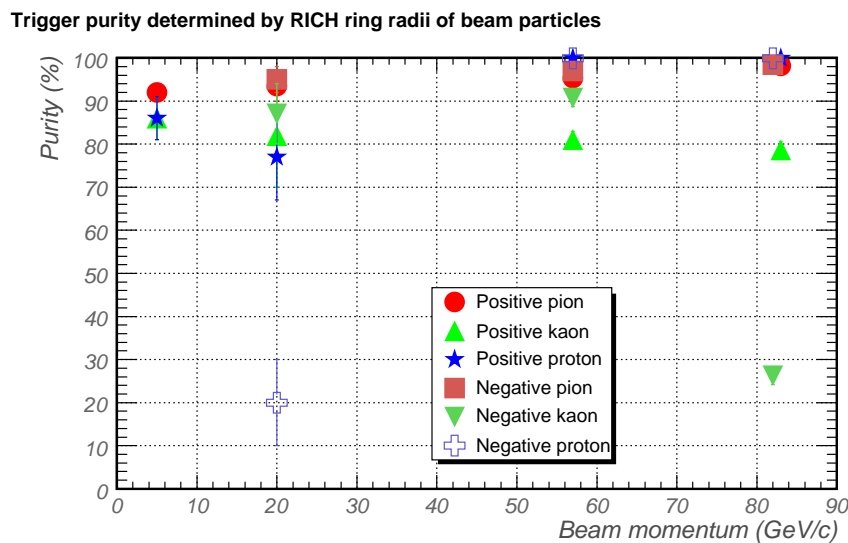

Figure 3.3: Trigger particle tag purity as measured by the RICH.

c) Small efficiency for two-particle final states where one particle is soft.

To address the problems of ${ }_{\widehat{N}} \mathrm{DC} 1$ interaction trigger, a scintillator interaction trigger was built. Both triggers were used in order to measure systematic effects of each interaction trigger.

\subsubsection{DC1 Trigger}

Drift chamber preamplifier cards, each serving 8 wires, are mounted on the chamber. Four preamplifier cards are connected to one discriminator. Each discriminator output 32 ECL signals, one per wire, and 4 ECL OR signals, one for each cluster of 8 wires. The cluster OR signals are sent to majority logic units (LeCroy 4532 CAMAC module) to create a multiplicity interaction trigger, called iDC. The trigger fires if at least 2 different clusters fire in at least 3 out of 4 planes.

\subsubsection{Scintillator Interaction Trigger}

The scintillator interaction trigger $\left(\right.$ Scint $_{N}[6]$ is much simpler than the iDC. A $3.175 \mathrm{~mm}$ thick $5.40 \times 7.62 \mathrm{~cm}$ piece of scintillator is placed $\approx 1.5 \mathrm{~cm}$ after the target. The scintillator has interaction length of $81.9 \mathrm{~g} / \mathrm{cm}^{2}$ and density of $1.032 \mathrm{~g} / \mathrm{cm}^{3}$, so its thickness is about $0.4 \%$ interaction length. Clear fibers collect light from the two shorter sides of the scintillator. The other ends of fibers are closely packed onto the face of Burle 83054H PMT. 
By safety rules, in order to operate the trigger with hydrogen target, all high voltages (i.e. PMT) had to be at least $3 \mathrm{~m}$ away from the target, hence calling for rather long fibers. Even with $3 \mathrm{~m}$ of fiber, the counter gets about 73 photo-electrons per minimum ionizing particle (mip).

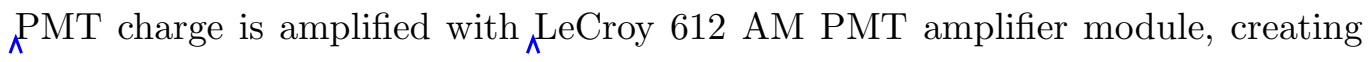
two copies of the signal. One copy goes directly to an ADC, second copy goes to an analog splitter. The outputs of the splitter are discriminated with two different thresholds, creating $\operatorname{SciHi}_{\wedge}(\approx 3 \mathrm{mip})$ and SciLo $(\approx 1.5 \mathrm{mip})$ digital signals.

The problem with SciLo signal is that Landau tail of single-particle energy deposition dominates the count rate from ${ }_{\Lambda} 1 \%$ interaction length target. At the threshold level of SciHi, only $\approx 1 \%$ of single-particle states deposit enough energy to create the trigger signal, thus yielding trigger purity of $\approx 50 \%$

\subsection{End of Spill and Calibration Triggers}

Each accelerator beam spill was followed by an end of spill trigger and a few pulser calibration triggers.

Upon receipt of end of spill trigger, detectors were not triggered, only the following actions were taken:

1. Scalers for the entire spill were read out and cleared;

2. A record was written to the database with the number of different triggers recorded in the spill;

3. Buffered data transferred from ${ }_{\Lambda}$ PowerPCs to the DAQ server.

On calibration triggers, detectors were triggered by a pulse generator. These triggers were used to calculate pedestals and to identify hot channels.

\subsection{Veto Counter}

In order to limit the effect of beam halo coming from the primary target in secondary beam configuration, a $35 \mathrm{~cm} \times 40 \mathrm{~cm}$ scintillator paddle with a $4.32 \mathrm{~cm}$ diameter hole is mounted on a plate of aluminum $140 \mathrm{~cm}$ upstream of the target. Two PMTs are 


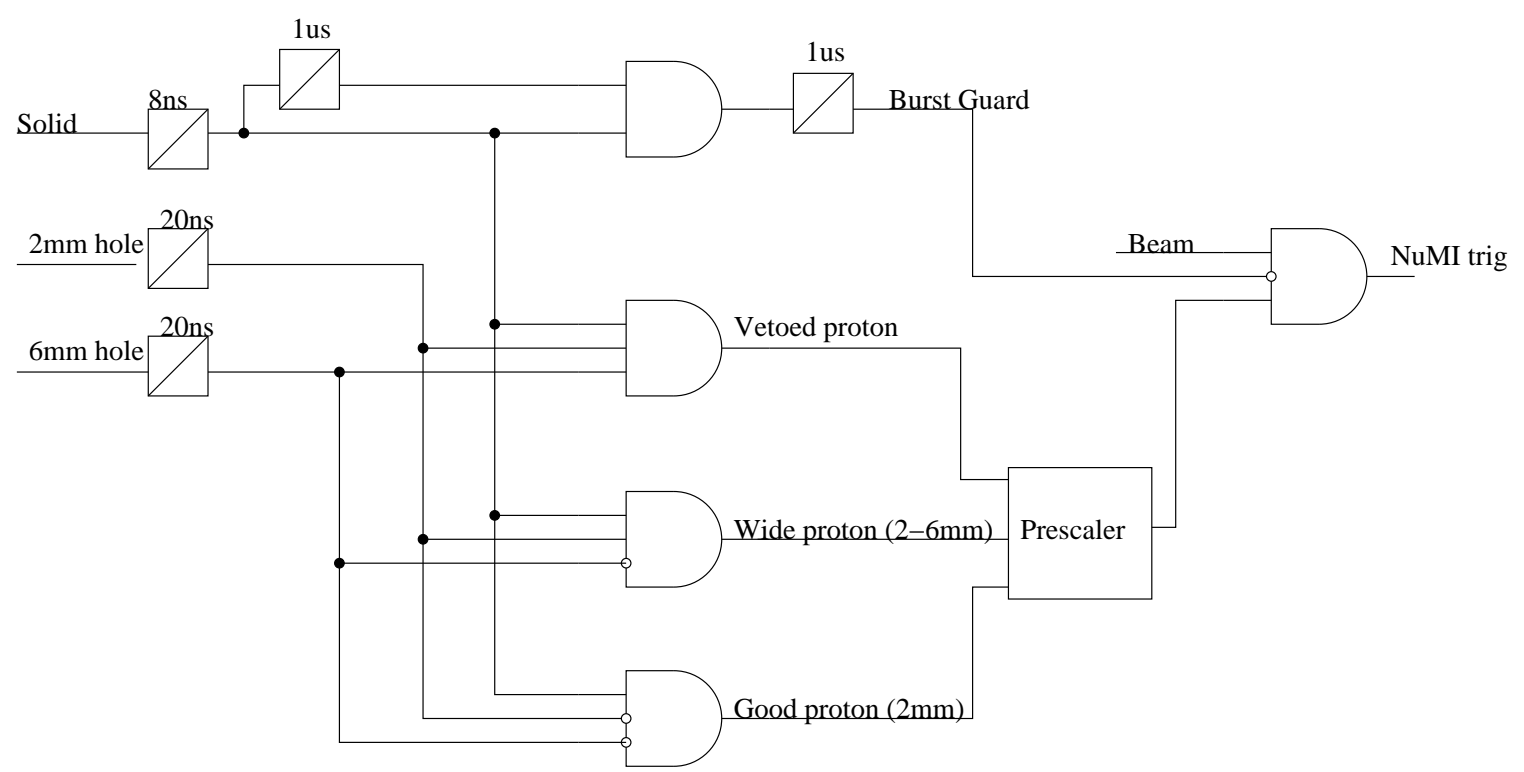

Figure 3.4: Logic for ${ }_{N} \mathrm{NuMI}$ trigger. Trigger rate in each of 3 different regions could be scaled down, so that even with poorly tuned beam, we could ensure that most triggers were taken with protons on target.

attached to the sides of the veto scintillator through light guides, and the logical AND of the two PMTs form an 80 ns veto signal to reject events with particles outside of the hole.

\subsection{NuMI Trigger}

When data was taken with the MINOS target, no interaction trigger was required since the target is 2 interaction lengths. However, the beam in MC7 was larger than the NuMI beam, so a dedicated trigger counter was built in order to make beam phase space at MIPP similar to ${ }_{\wedge}$ NuMI beam. The counter consisted of three pieces of scintillator: solid, with ${ }^{2} \mathrm{~mm}_{\lambda}$ diameter hole in the center, and with $6-\mathrm{mm}_{\lambda}$ hole. The paddles with holes were used to veto particles that were too far from the beam center, thus ensuring that most data is taken under conditions resembling the NuMI proton beam. The logic for this trigger is shown in Figure 3.4

In addition to vetoing wide particles, the trigger had a $1 \mu \mathrm{s}$ "burst guard" which prevented the trigger from firing if two protons were separated by less than $1 \mu \mathrm{s}$. Burst guard was necessary in order to prevent pileup. Since almost all protons interact in the 
target with fairly large multiplicities, two events, one on top of one another ${ }_{\wedge}$ would be very hard to reconstruct in the TPC. 


\section{Chapter 4}

\section{Data Acquisition System}

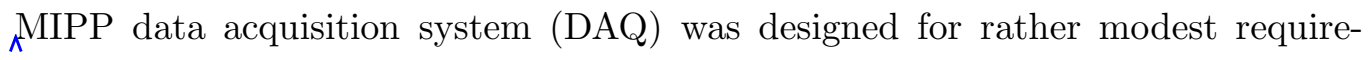
ments. Trigger rate was limited to about $60 \mathrm{~Hz}$ due to the time it took to zero suppress TPC data. Around $90 \%$ of the data came from the size of the TPC event, $100 \mathrm{kB}$ on average. Initial design specifications were to read out all of the detectors in a mode where beam was delivered in $1 \mathrm{~s}$ spills, 20 spills per minute, or $2 \mathrm{MB} / \mathrm{s}$. MIPP DAQ was able to keep up with the data rate, which did not exceed fifteen $600 \mathrm{~ms}$ spills per minute (see Chapter 5).

Figure 4.1 shows the overall schematic of the data acquisition system. Computers which made the experiment work included ${ }_{\Lambda}$

- ${ }_{\Lambda} \mathrm{DAQ}$ server which had 2 network cards, ${ }_{\Lambda}$ was connected to the public net and to the private subnet.

- Six VME PowerPC's (PPC), connected to the DAQ server through 100 Mbit network were able to access VME and CAMAC readout electronics and send data to the server.

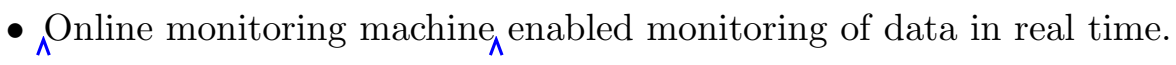

- High Voltage machine ${ }_{\Lambda}$ was used to control and monitor all PMT and TPC high voltages (wire chamber high voltages were manually controlled).

- ${ }_{\wedge}$ Database server ${ }_{\wedge}$ which ran ${ }_{\wedge}$ PostgreSQL database where all online information was stored.

This chapter describes all the online software that was running on all of these machines. 


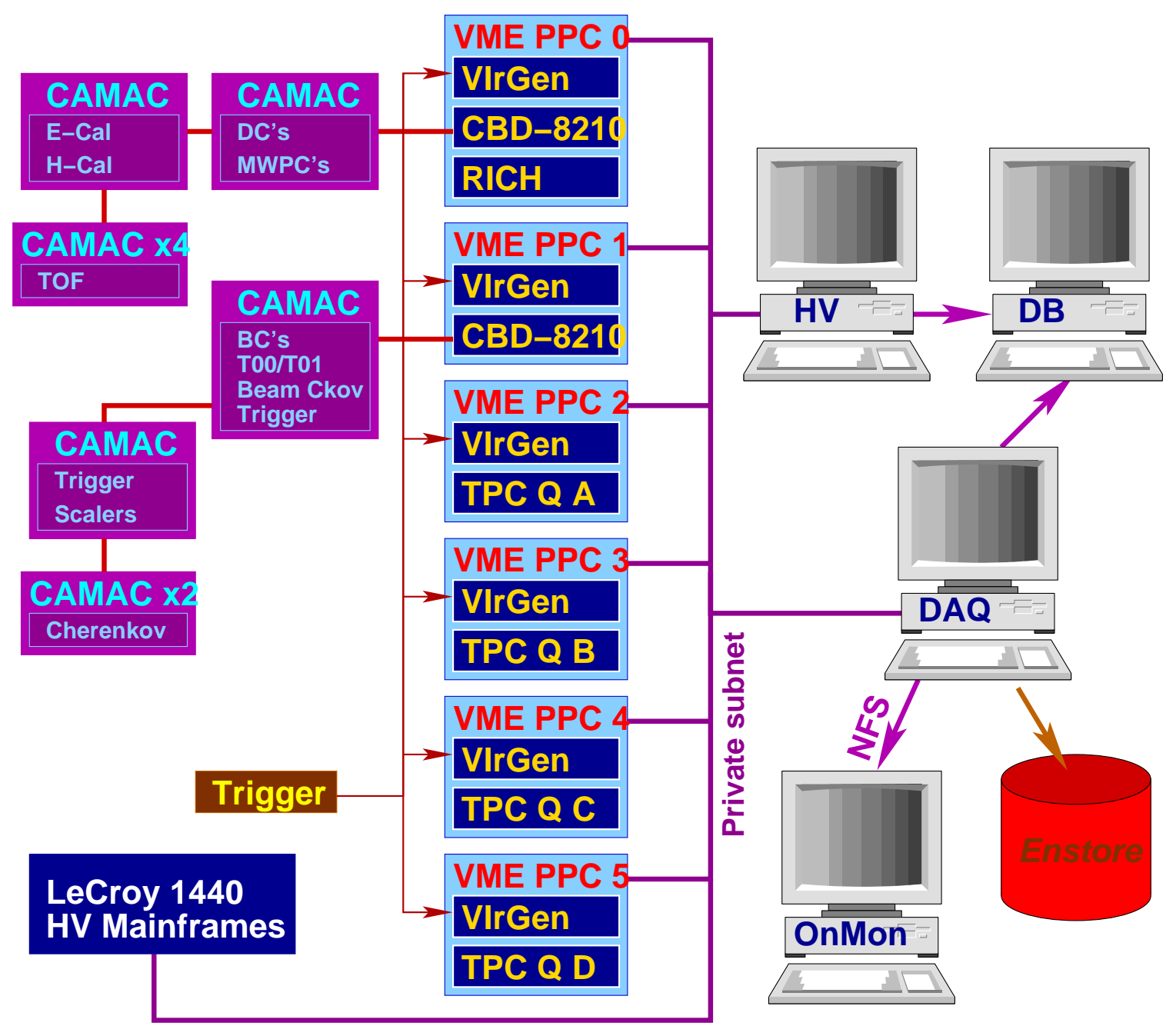

Figure 4.1: Schematic of data acquisition setup. Of the six VME PowerPCs, four were dedicated to control and read out of the TPC quadrants, and the other two read out the rest of the detectors, mainly CAMAC electronics. Readout processes were triggered through VME interrupts delivered by VIrGen ${ }_{\lambda}$ boards. High voltage mainframes connected to private subnet as well, were controlled and monitored by HV machine. Detector data were written out to dedicated files, and online monitoring data were stored in PostgreSQL database. Online monitoring was done by exporting data disks through NFS. Finally, data were regularly shipped to FNAL robot tape storage (Enstore). 


\subsection{VME and CAMAC Interfaces}

At the time when MIPP DAQ was designed, one of the most affordable ways to access VME bus was to run Linux on VME PowerPC's made by Motorola. MIPP required 6 MVME 2432 computers: 4 dedicated to TPC readout and 2 more to read out all other detectors. Those machines had $350 \mathrm{MHz}$ processors and $256 \mathrm{MB}$ of memory. They were placed into $6 \mathrm{U}$ VME crates so as to have direct access to the VME bus. $\wedge^{\text {On-board }}$ Tundra Universe II chip provided access to the VME bus. MIPP used a small subset of its capabilities for VME transactions: A24/D16, A16/D16, A32/D32 data transfers, and interrupt handling.

Most detectors were configured and readout through CAMAC electronics. Two CBD 8210 CAMAC branch drivers made by Creative Electronics Systems S.A. were used to drive two CAMAC branches with a total of 12 crates.

\subsubsection{Linux on PowerPC}

PowerPC's have two links to the outer world: $\Lambda^{9600-b a u d ~ d e b u g}$ port and $\Lambda^{100-}$ baseT ethernet connection. Having no hard drive, the computer had to be booted from a remote machine. The kernel, loaded through $\operatorname{tft}_{\boldsymbol{\Lambda}}$ protocol, would know the location of the file system mounted through NFS. On-board flash memory could be programmed to select the server and location of the kernel file on the server.

Linux kernel 2.2.12 and the file system based on glibc-2.2.5 was compiled from scratch using gcc-2.95.3. We inherited a file system compiled for kernel 2.1.24 with egcs. Since the compiler and kernel had significant changes and improvements, it was necessary to upgrade the operating system. I followed Linux From Scratch[26, 3] recipe to rebuild the file system.

At the time the file system was built, it seemed that having RedHat Package Manager (RPM) on the operating system would be necessary to facilitate system administration. However, since PowerPC's were on the private sub-net, security was not an issue, and system maintenance was much simpler than for computers exposed to the internet. The system had to be supported on 6 computers only, so most packages compiled from source were not re-installed or incorporated into ${ }_{\Lambda}^{\mathrm{RPM}} \mathrm{\text {database. }}$

Even though the number of packages is not nearly as large as on most modern operating systems, having to serve 6 systems from the DAQ server required a significant 
amount of disk space. To reduce the total size of the systems, debugging symbols were stripped from executables and system libraries. Furthermore, unlike system binaries and libraries, one common /usr/share directory was exported to the PPC's and MIPP online software resided in /usr/local, common to all 6 computers.

\subsubsection{Handling interrupts in the kernel}

${ }_{\Lambda}$ Kernel module to drive ${ }_{\Lambda}$ Tundra II chip $\boldsymbol{\Lambda}_{\boldsymbol{\Lambda}}$ written by Gabriel Paubert could handle $\mathrm{I} / \mathrm{O}$, but could not deliver interrupts into user space. In order to simplify debugging, we wanted to write all online software in user space. Performance loss due to this inefficiency could be tolerated because TPC electronics rather than VME readout was the bottleneck. In order to deliver interrupt $t_{\Lambda}$ into user space, a modification to the module was necessary.

Final design was implemented using semaphores. ${ }_{\Lambda}$ User's program would increment the value of the semaphore, release VIrGen busy (see Section 4.1.4), and then block until the semaphore value was reset to zero by the kernel. This design avoided race condition where the program would miss an interrupt, but as we found out, had a different flaw. Since Linux is not a real-time operating system, in rare instances when the kernel was busy, it would fail to unblock the program through the semaphore. To circumvent this problem, mdd (see Section 4.2.4) had a dedicated thread which checked the status of the hardware busy for that VME crate and would signal the blocked thread if the kernel failed to unblock it. However, sustained trigger rates of multiple $\mathrm{kHz}$ would cause kernel panic and crash the PowerPC. For lack of need and shortage of time, that problem had not been resolved since production data acquisition rates did not exceed 100 triggers per second.

If time and manpower permitted, interrupt handling would be re-dong through poll() system call rather than through semaphores. We are quite certain that a significant improvement in kernel stability could be achieved that way.

The DAQ system was configured to expect two interrupt types:

- Data trigger on VME interrupt 2;

- End of spill trigger on VME interrupt 3.

The difference between the two interrupts was that on end of spill trigger only scalers were read out in the trigger process and all other processes sent empty data messages. 


\subsubsection{CBD 8210}

CBD 8210 is a double height VME card allowing a parallel CAMAC branch with up to 7 crates to be driven through VME. Presently, the boards are quite difficult to find, and MIPP was able to obtain only 3: two for active use and one spare. The board characteristics include

- 16 and 24-bit CAMAC transfers,

- Two external VME interrupts,

- Comprehensive LAM (look at me) handling,

- Addition of a DMA module.

Our primary objective of 16 and 24-bit transfers was easily accomplished as it is well documented in the manual [12]. Before ${ }_{\Lambda} \operatorname{VIrGen}$ boards were ready, we were also using the two interrupts to trigger readout. Unfortunately, we were not able to get DMA transfers to work, although the primary reason was the lack of need to boost CAMAC transfer performance: ${ }_{\Lambda}^{\mathrm{TPC}}$ was limiting data readout rates and it was readout directly through VME.

\subsubsection{VME Interrupt Generator}

VIrGen (VME Interrumpt Generator) boards were developed at Harvard to

1. Convert trigger NIM signal into VME interrupt,

2. Time stamp each trigger to ensure that event pieces recorded on different PowerPC's corresponded to one physical event,

3. Ensure that Linux kernel does not miss an interrupt,

4. Hold off further triggers until a process on the PPC signals that readout is complete.

The board was outfitted with four inputs for four different VME interrupts (1-4). All decisions were made by XILINX FPGA.

The first objective was accomplished by feeding the NIM signal into a comparator, its output connected to FPGA input. If that interrupt channel was not holding busy, then 
${ }_{\wedge} \forall$ ME interrupt on $\wedge_{\wedge}$ corresponding channel was initiated and handshake was completed with the PowerPC. All boards were running off the accelerator RF clock and 32 -bit time stamp was recorded on the clock cycle that fired the interrupt. Synchronization of timestamps across the 6 boards was achieved by resetting time stamp to 0 at the beginning of every run.

One of the issues that we encountered with Linux interrupts was that occasionally (apparently when the load was high), an interrupt would be missed. In order to solve that problem, VIrGen would keep resending VME interrupt until a process on the PowerPC would acknowledge receipt of the interrupt by writing to a register on the board. Frequency of repeating interrupts could be controlled through a register.

In order to synchronize all VME crates, once an interrupt was received, VIrGen set the corresponding TTL busy output high. Once readout was complete, a process on the PowerPC had to clear the busy. Externally formed logical OR of all busy signals inhibited further experimental triggers.

\subsection{Online Software}

\subsubsection{MippIo}

From the start, it was decided that offline analysis would be done in ROOT, but it was suspected that ROOT I/O would not be fast enough to cope with TPC data volume, so a dedicated I/O library was written.

MippIo was designed to write data in a structured byte-packing format organized into blocks. Each block had a 12-byte header containing block ID, version, and total block size. MIPP raw data file consisted of a file header block followed by any number of event blocks and concluded with an end of file block. In turn, each event block consisted of a number of detector blocks. Each detector block contained data as it was read from VME or CAMAC, without any pre-processing done on the PowerPC's or in the event builder.

\subsubsection{Run Control and Message Passing System}

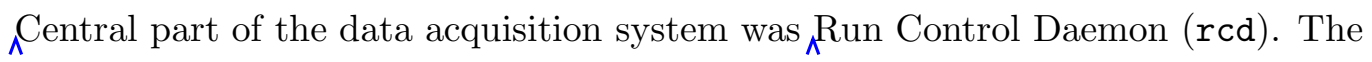
process had to perform the following tasks

- Maintain the overall state of the DAQ system; 


\begin{tabular}{|c|c|c|c|}
\hline Class name & Senders & Receivers & Description \\
\hline RCMsgConnect & All & All & $\begin{array}{l}\text { Message sent by a client to request } \\
\text { new connection and response by } \\
\text { rcd to acknowledge new connec- } \\
\text { tion }\end{array}$ \\
\hline RCMsgControl & All & $\begin{array}{l}\text { Detectors, } \\
\text { event builder, } \\
\text { rcd }\end{array}$ & $\begin{array}{l}\text { Generic message sent to start and } \\
\text { stop runs, and the primary way } \\
\text { for GUI to make rcd to act }\end{array}$ \\
\hline RCMsgDataDir & rcd & GUI & $\begin{array}{l}\text { List of directories to choose from } \\
\text { for writing data }\end{array}$ \\
\hline RCMsgEvBStatus & Event builder & rcd & $\begin{array}{l}\text { Update rcd of event builder sta- } \\
\text { tus }\end{array}$ \\
\hline RCMsgMddStatus & mdd & $\operatorname{rcd}$ & Update rcd of mdd status \\
\hline RCMsgPrescaleList & GUI, rcd & GUI, rcd & $\begin{array}{l}\text { Changes and updates of prescale } \\
\text { settings }\end{array}$ \\
\hline RCMsgProcRel & $\begin{array}{l}\text { Detectors, } \\
\text { event builder, } \\
\text { mdd }\end{array}$ & $\mathrm{rcd}$ & $\begin{array}{l}\text { Inform rcd of the release used to } \\
\text { compile a given binary }\end{array}$ \\
\hline RCMsgRunConfig & GUI, rcd & & $\begin{array}{l}\text { Run configuration, including } \\
\text { prescale settings, target, enabled } \\
\text { detectors }\end{array}$ \\
\hline RCMsgStatus & rcd & GUI & $\begin{array}{l}\text { Update GUI of current status of } \\
\text { the DAQ }\end{array}$ \\
\hline RCMsgTarget & $\operatorname{rcd}$ & GUI & $\begin{array}{l}\text { Pass a list of available targets to } \\
\text { rcd }\end{array}$ \\
\hline
\end{tabular}

Table 4.1: Message classes implemented in run control.

- Process operator requests to start and stop data acquisition;

- Respond to exceptions and errors in the system.

These objectives were achieved by passing messages between different processes across the network.

$\aleph^{M}$ Message passing system was implemented through stream sockets, allowing maximal flexibility and expansion of the DAQ system. A number of different messages were implemented (see Table 4.1), each having a 20-byte header containing message id, its number, size, second, and microsecond when the message was packed.

Upon start-up, rcd would open a socket to listen to incoming connections and would go into an infinite loop which polled all known sockets for activity. Once an event occurred on a socket, it would be processed by rcd. New connections were classified as event 
builder, mdd, GUI, or detector process, and messages were processed sequentially. Since the number of connections was small (typically 8) and response time to nearly all messages did not involve much CPU time, this simple architecture worked very well.

Once all the software on all machines was up and running in idle state, it was up to the operator to set the run conditions (trigger prescales, set of detectors, etc) through GUI and start a run. GUI would send run configuration to rcd and follow it up with control message to start a run. At that point, rcd would

1. Figure out the current target and beam momentum by reading slow control devices;

2. Save run conditions to the database;

3. Send a start run message to event builder;

4. Wait for event builder to respond that the run has been started;

5. Send a start and pause run message to mdd's;

6. Wait for every mdd to start up its detector processes;

7. Send a start run message to all mdd's.

If any of the steps failed, run was terminated. In this process, only the first step would block the cycle of listening to incoming messages.

Requests to stop a run were processed in reverse:

1. Send a stop run message to all mdd's;

2. Wait for all mdd's to terminate child processes;

3. Send a stop run message to event builder;

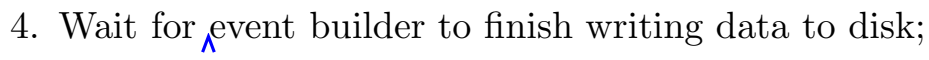

5. Save run statistics to the database.

Unlike requests to start a run, any process could send a request to stop a run if it encountered an error. Alternatively, if any of the processes involved in the run would stop responding,

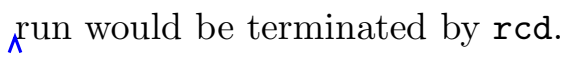




\subsubsection{Event Builder}

Event builder is a FNAL Computing Division product that MIPP customized to our needs. It has to be able to receive parts of events (called subevents) from different sources, assemble them into an entire event, and write it to disk. At the beginning of the run, the process would be configured to expect a certain number of connections: one for each detector and one for each mdd involved. In order to synchronize parts of an event, each process had to be triggered to send a message to event builder even if there was no data read out. Events were assembled once all subevents were received.

Additional task of the event builder was to cut long runs into shorter subruns. A MIPP run was defined as a set of data with one system configuration (trigger scale down factors, high voltage, etc). A subrun was defined as a file with size less than 1 GB or not more than 30 minutes in duration if it contains at least 5000 events. Having multiple shorter files rather than one gigantic file facilitated data handling and expedited analysis on the batch farm.

\subsubsection{MIPP DAQ Daemon}

On each PowerPC, one process was

- Responding to rcd messages;

- Starting and stopping detector processes;

- Receiving and handling interrupts;

- Handling exceptions and errors during data taking.

It was named mdd because initially the DAQ was expected to rely on a different message passing system, and this would be the only "daemon" running. In a way, its functionality was similar to rcd: at the beginning of the run, all necessary processes were started, monitored during the run and terminated at the end of a run. The difference was that it responded to rcd and was not aware of the state of the entire DAQ system.

mdd was a multi-threaded program: one thread handled control messages and two "trigger" threads were handling interrupts on the two interrupt channels. As mentioned earlier, VME interrupts were handled by $\wedge^{k e r n e l ~ a n d ~ p a s s e d ~ t o ~ u s e r ~ p r o c e s s e s ~ t h r o u g h ~}$ semaphores. Once a trigger thread received an interrupt, it would lock a mutex $x_{\mathcal{k}}$ and one by 
one get each of the detector processes to read data. Inter-process communication was also implemented through semaphores. It was necessary to have only one detector reading data at any given time because CAMAC operations were not done atomically: one VME operation transferred the data and a second operation read back the status of the last transfer. Hence if all detectors were read out simultaneously, a race condition was possible where a process would find out the status of a wrong data transfer.

An alternative design would be to link all detector libraries into mdd and avoid context switching. This approach was not pursued because we thought that better stability would be achieved if readout was done in a separate processes.

Trigger handling was done differently on the TPC crates than on the other two crates. The reason is that TPC events were interleaved, i.e. a trigger could proceed forward before the event was read out. Thus, on the TPC crates triggers were handled with the following sequence of events:

1. When triggered, ${ }_{\wedge}$ IrGen board would set a busy and hold it;

2. Kernel would set respective semaphore to zero;

3. mdd would wake up and wake up TPC process;

4. TPC process would wait for the data to be ready for readout, switch the buffer where next event was written;

5. TPC process would increment semaphore value, release VIrGen busy and then read data out.

The other two crates had a slightly different sequence of events: mdd would cycle through all detectors, increment semaphore, and then reset VIrGen busy. These sequences were safe against race conditions.

One of the features we found very useful was measuring readout time of every detector. This helped us to identify bottlenecks in the beginning and at a later stage was a useful debugging tool: if detector was taking much more or much less time than usual to read its data, it typically indicated a problem with the electronics, high voltage, or low voltage supplies. 


\subsubsection{Detector Processes}

Detector processes had to fulfill a very specific role: connect to event builder, configure detector at the beginning of a run, read and buffer data for every trigger, send data to event builder on every end of spill trigger. Each process had two threads, one for each interrupt type.

\subsubsection{Database Logger}

In order to avoid building Postgres libraries on the PowerPC and most importantly to be able to host database on a dedicated computer rather than the DAQ server, dbLogger interface was written so that executables running on the PowerPC's could add and modify entries in the database. Logger daemon $n_{\lambda}$ was running on the DAQ server, it listened to incoming connections from the PowerPC's and passed SQL commands to the Postgres server without parsing thexm.

The main use for dbLogger was to store the number of spills and triggers recorded in a given run.

\subsubsection{Online Monitoring}

An integral part of the data acquisition system, online monitoring was essential debugging tool and gave shifters ability to verify in nearly real time that the data written to disk was meaningful. Having the program was also essential to debug issues in MippIo and detector readout code.

Monitoring was running on a dedicated computer which had the data disks mounted through NFS. The program was constantly scanning the disks for new data files and kept updating a number of histograms for all detectors. The executable was written using ROOT for both histograms and graphical user interface. Important part of online monitoring was writing files out at the end of each run so that histograms from different runs could be quickly compared at a later time.

\subsubsection{Software Release System}

Entire experiment depended on the DAQ system, therefore some order was brought into software development cycle by compiling frozen releases. Rigorous testing was done before a release was made, and at any point in time, the most recent and the previous stable 
releases were available to operators. Release of every DAQ executable for every run was stored in the database so that at a later time potential detector or data integrity problems could be easily linked to software release.

\subsection{Slow Controls}

Environment in the experimental hall as well as settings and readbacks of devices were controlled and continually monitored by computer. These included gas pressures, temperatures, magnet current settings, and high voltage settings.

\subsection{1 iFix}

Intellusion iFix system was used as a front-end for APACS control. It was used to

1. Read air and gas pressures, multiple temperature sensors, humidity sensor;

2. Control the pressure in beam Cherenkov counters;

3. Control the flow of gas through threshold Cherenkov counter;

4. Act as an interlock on the RICH cooling system;

5. Monitor chamber gas flow;

6. Monitor gas mixing for drift chambers;

7. Monitor and control $\Lambda_{\Lambda}^{\text {cryogenic target. }}$

$\Lambda^{X M L-R P C}$ server was running on the Windows PC where iFix was running to provide a way to store variables to the Postgres database and for DAQ to find out the status of cryogenic target.

\subsubsection{ACNET}

ACNET is Accelerator Control NETwork developed at and supported by FNAL. We relied upon ACNET to

1. Control and monitor secondary beamline dipole and quadrupole magnets;

2. Control momentum collimator slit width; 
3. Monitor beam profiles;

4. Monitor spill structure;

5. Control and monitor analysis magnet currents;

6. Monitor Hall probes installed in the apertures of analysis magnets;

7. Control and monitor RICH high voltage supplies;

8. Control beam Cherenkov mirror positions;

9. Monitor wire chamber and EM calorimeter voltages and currents;

10. Monitor spill intensity;

11. Monitor hall and target temperature;

12. Control target wheel position;

Similarly to iFix, we used XML-RPC server provided by ${ }_{\wedge}$ FNAL Beams Division to control devices and record their state into the database.

\subsubsection{High Voltage}

High voltages on PMT's and TPC anodes were controlled through two LeCroy Research Systems 1440 systems. In order to control the mainframes from the counting house $100 \mathrm{~m}$ away, Equinox serial hub was connected to the private sub-net. This device enabled access to serial devices over 100 Mbit network.

Once high voltages were set, the system would continually cycle through all known channels, store the readback voltage to database and verify that the channel did not trip. This was especially important for TPC anodes which did occasionally trip. Prompt detection of trips ensured that as little data as possible was lost. 


\section{Chapter 5}

\section{Beamline}

\subsection{Accelerator}

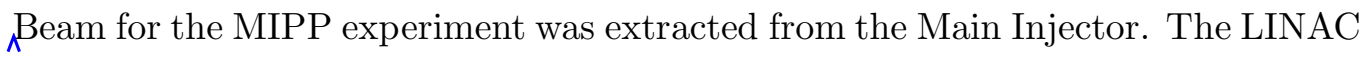
followed by Booster accelerate batches of protons to $8 \mathrm{GeV}$ kinetic energy, and the Main Injector RF cavities accelerate protons to $120 \mathrm{GeV} / c$. Up to 7 Booster batches can be injected into the Main Injector to be accelerated simultaneously. Protons for the MINOS experiment or for $\bar{p}$-production for the Tevatron collider are extracted in single turn. ${ }_{\Lambda}^{\mathrm{MIPP}}$ spectrometer required resonant extraction out of the Main Injector to reduce instantaneous rate. Beam extracted towards the Switchyard; passed through the A-section of the Tevatron $\operatorname{ring}_{\bar{\lambda}}$ and at the Switchyard beam ${ }_{\lambda}$ could be split between the Meson Test area, the Meson Center (MC) area, and the beam dump. MIPP spectrometer occupied MC7 hall.

The experiment was approved under condition that it would not impact the accelerator program by more than $5 \%$. In practice that meant that we were not to slow down $\bar{p}$-production by more than $5 \%$. At the beginning of the MIPP run, this translated into extracting all but one Booster batch to $\bar{p}$-production, and slowly extracting the last batch to MIPP. Since resonant extraction increased the length of the Main Injector time cycle, while the $\bar{p}$ stack was small (hence stacking rate high), MIPP received about six $600 \mathrm{~ms}$ spills per minute. Once the $\bar{p}$ stack grew and stacking rate slowed down, MIPP was receiving up to 15 spills per minute.

In March 2005, the NuMI beamline started receiving proton beam for the MINOS experiment, and in April 2005, the accelerator complex operation was changed so that MINOS and $\bar{p}$-production co-existed with single-turn extraction to each destination, and a 


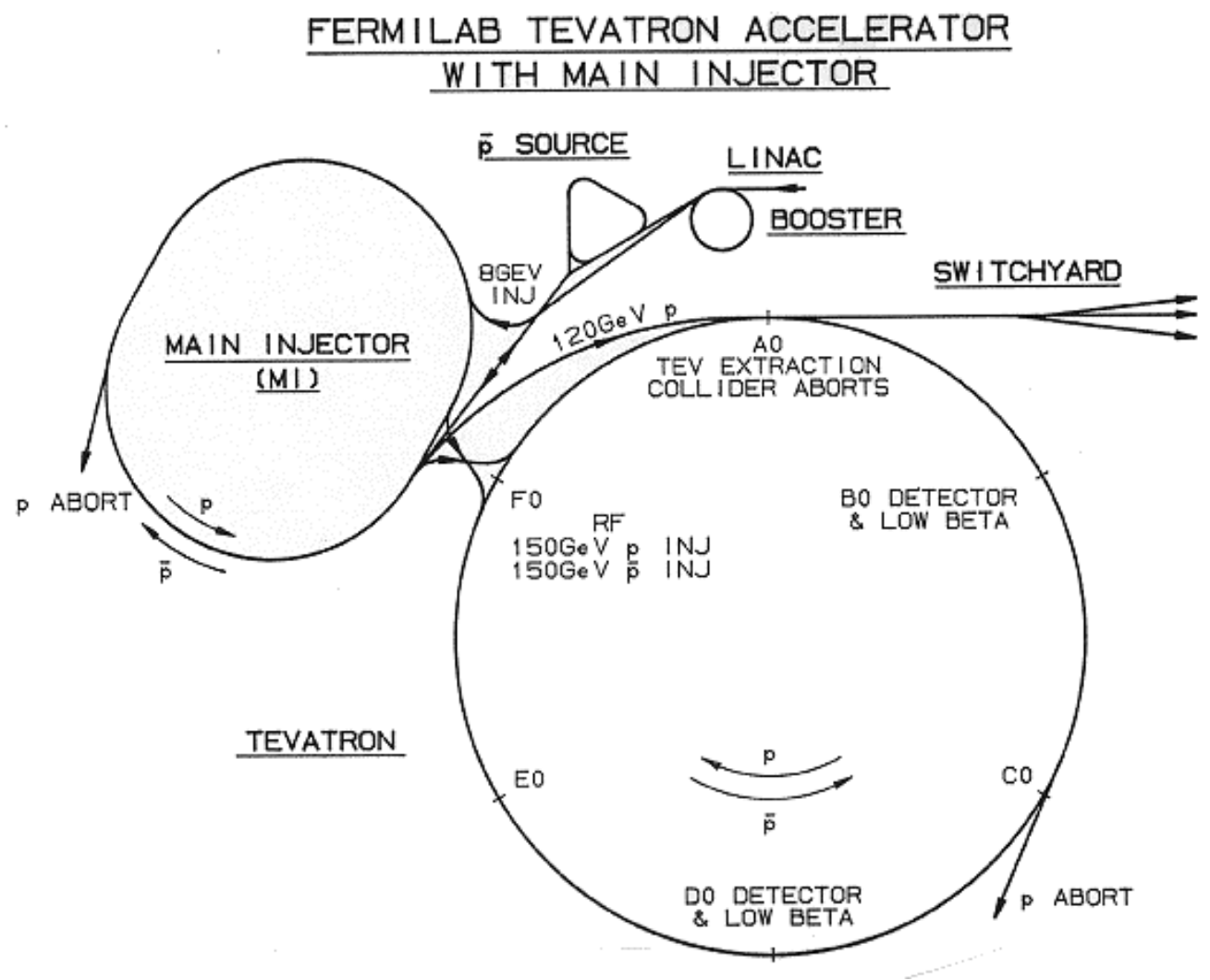

Figure 5.1: Schematic of the FNAL accelerator complex. $\wedge^{\mathrm{MIPP}}$ experiment was located in the beamline past Switchyard. 
4 second spill was extracted to the Switchyard once every 2 minutes. MIPP operated under these conditions through February 2006.

The MIPP beamline was operated in two distinct modes:

- $120 \mathrm{GeV} / c$ primary protons

- $5-90 \mathrm{GeV} / c$ secondary particles produced on a copper target.

\subsection{Primary Beam}

The minimum rate at which protons could be extracted from the Main Injector was about $10^{8}$ particles per second. Radiation safety mandated that the experimental hall was to receive no more than $10^{6}$ particles per second. Under normal circumstances this limitation did not affect data rates, since considerations of pileup and build up of space charge in the TPC made it impossible for the experiment to handle more than $10^{5}$ particles per second, more realistically $3-5 \times 10^{4}$. In short, proton intensity had to be reduced by at least 3 orders of magnitude in the beamline between the Main Injector and MC7 hall. This was achieved by blowing up the beam size before passing it through a pin-hole collimator, and then focusing the beam on the experimental target.

For the NuMI target running, beam intensity was further reduced to about $10^{3}$ particles per 4 second spill. This was necessary because with a 2 interaction length target, $90 \%$ of incident particles interact, hence higher rates would create unacceptable level of pileup.

\subsection{Secondary Beam}

At the entrance to $\mathrm{\wedge} \mathrm{MC} 6$ area, $120 \mathrm{GeV}$ protons were focused onto a $0.5 \times 0.5 \times 20 \mathrm{~cm}$ copper target, located $97 \mathrm{~m}$ upstream of the experimental target. The copper target is about 2 interaction lengths, hence a tenth of the primary beam was not interacting and was dumped into a series of concrete blocks. Beam was striking the target at $1.3^{\circ}$ with respect to the horizontal, and 4 dipole kicks - two before and two after the slit collimator bringing horizontal beam onto the experimental target.

In order to minimize $d p / p$, large dispersion had to be achieved at the collimator. Six quadrupole magnets - three before and three after the collimator - enabled us to increase 
dispersion and then focus the beam on the target.

An estimate of particle production, gives RMS transverse momentum of about $400 \mathrm{MeV} / c$. Physical transverse dimension of the target is $\pm 2.5 \mathrm{~mm}$. Hence, ${ }_{\Lambda}^{\mathrm{RMS}}$ emittance of the secondary beam varies from $\sim 200 \mathrm{~mm} \cdot \mathrm{mrad}$ at $5 \mathrm{GeV} / c$ down to $\sim 11 \mathrm{~mm} \cdot \mathrm{mrad}$ at $90 \mathrm{GeV} / c$ (total emittance is roughly 4 times larger). The experimental target is $5 \mathrm{~cm}$ in diameter, and Beam Cherenkov operation requires less than 1 mrad divergence, so that the maximum total emittance needed by the experiment is $25 \mathrm{~mm} \cdot \mathrm{mrad}$. Hence, for all beam momenta, we had to cut down emittance at the momentum of interest.

\subsubsection{Optimizing Beam Quality}

Once MIPP detectors were up and running, we quickly realized that in order to take quality data with secondary beam, not only did we have to focus the beam on the target, but also reduce the amount of beam halo that results from scraping beamline elements. There is an infinite number of quadrupole current settings that provide a well focused beam, but most of them result in significant scraping in dipole beam pipes downstream of the collimator and degrade data quality in two ways:

- Faking interaction trigger, especially iDC trigger,

- Filling up TPC volume and tracking chambers with so many tracks that event becomes unreconstructable.

The effect on the interaction trigger was reduced by relying more heavily on the Scint trigger (see Section 3.3.2). ${ }_{\wedge}$ Veto counter further helped to reduce the fraction of unwanted events. The right way to improve operating conditions was to address the source of the problem by altering the beamline settings.

A complete model of secondary beamline was assembled and analyzed using OptiM software[30]. The model included realistic calculation of magnetic field in dipoles and quadrupoles based on measurements done at the FNAL Technical Division, and apertures of the beamline elements. ${ }^{1}$ Once this model was complete, one could quickly understand what was happening. Significant scraping occurred in the last dipole magnet, well after the collimator, with only one quadrupole magnet behind it (see Figures 5.2 and 5.3). Of all places where scraping could occur, this is one of the worst.

\footnotetext{
${ }^{1}$ The model assembled for use with Methodical Accelerator Design (MAD) Program lacked apertures, and hence was incapable of addressing the problems that MIPP experiment was facing.
} 


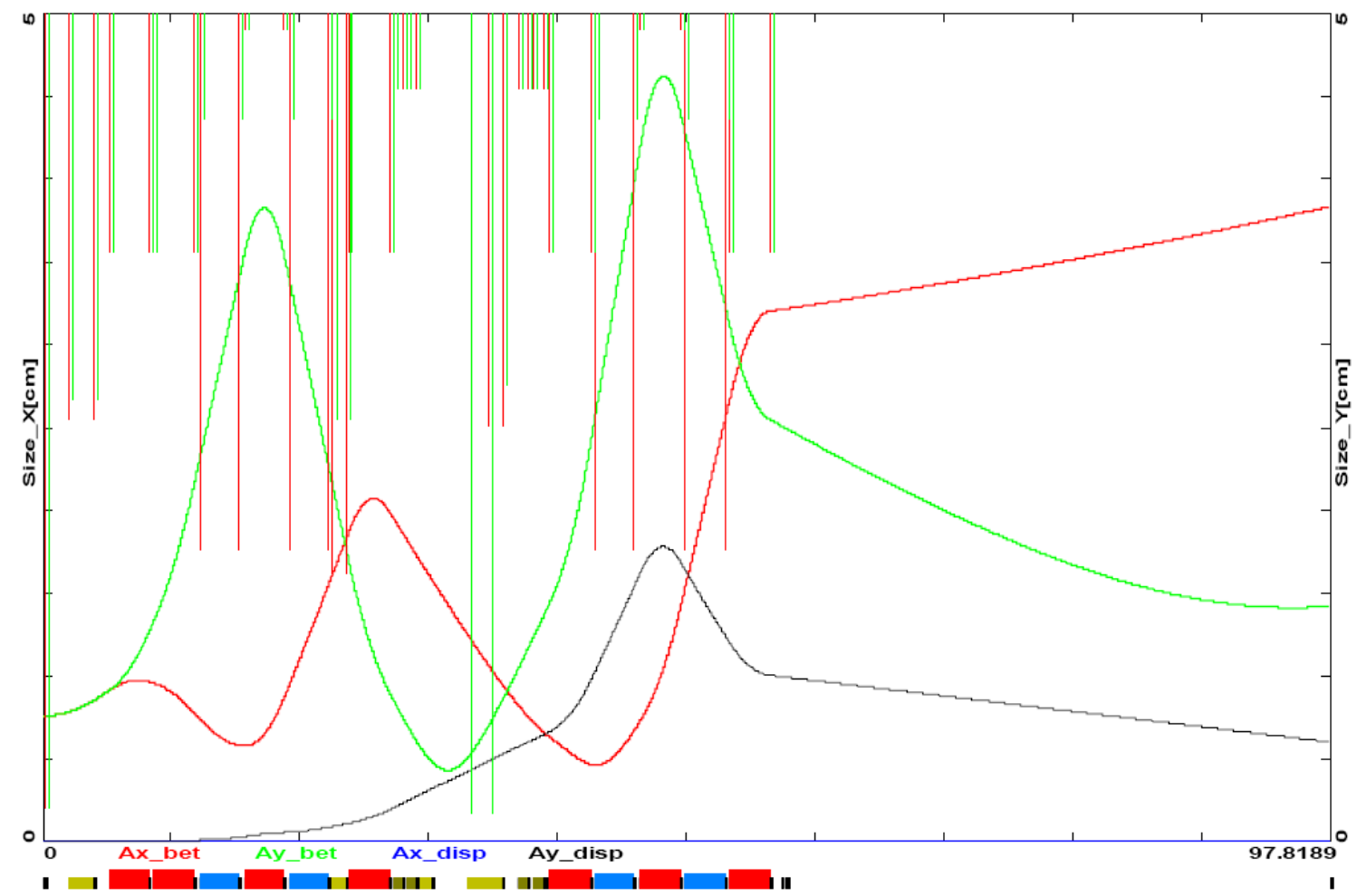

Figure 5.2: Top plot shows beam sizes ( $x$ in red, $y$ in green) and dispersion (black curve) superimposed on top of specified apertures (again, red for $x$ and green for $y$ ). Apertures exist in the model as line objects (rather than a box), hence aperture is specified at the front and back of every element. This plot can be produced with conservative assumptions (smaller than real-life emittance) about particle production on the copper target. Shown at the bottom are beamline elements: tallest red boxes are quads, medium blue boxes are dipoles, and khaki short boxes are scrapers and collimator. 


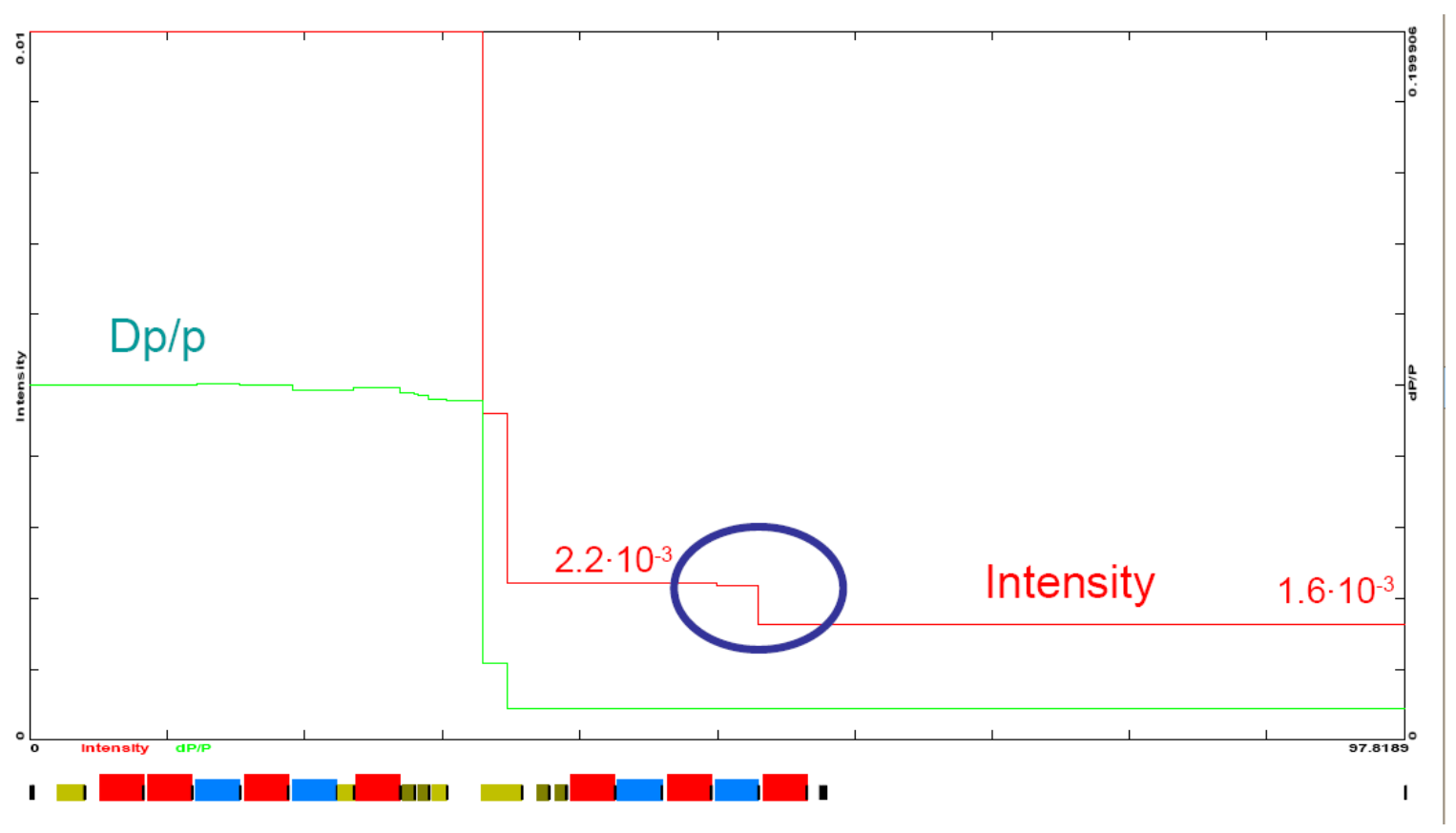

Figure 5.3: Intensity plot created by tracking a very wide $(10 \% d p / p)$ distribution of particles. OptiM tracking is very simple: a particle is lost if it hits an aperture, hence intensity is a step-function. As expected, most particles are lost at the collimator, still a large number hit the aperture of the last dipole (the loss highlighted by the oval). 
While it was no longer feasible to move beamline elements, in particular scrapers, the following approach helped select an acceptable solution leaving all elements in their positions.

Instead of modeling forward-going beam, beam parameters (emittance, $\beta$-function, and its derivative) were chosen at the experimental target and beam was transported backward ${ }_{\Lambda}$ and ${ }_{\Lambda}$ beamline settings ${ }_{\Lambda}$ chosen so that the beam was far away from apertures downstream of the collimator (in real beamline), touching every aperture upstream of the

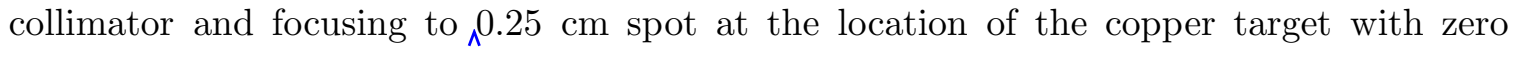
dispersion. The last 2 requirements are necessary to reflect reality of particles being produced from a finite-size target. Note that one does not need to mimic angular divergence of the beam at the secondary target because we are intentionally selecting a small subset of secondary particles, since full emittance at the secondary target is larger than what the experiment can handle.

Parameters which can be used to characterize beam at some location in $z$ are:

- Beam size $\left(x_{\max }, y_{\max }\right)$,

- Beam divergence $\left(\theta_{x, \max }, \theta_{y, \max }\right)$,

- Beam emittance $\left(\epsilon_{x}, \epsilon_{y}\right)$,

- Beta functions $\left(\beta_{x}, \beta_{y}\right)$,

- Derivative of beta function $\left(\alpha_{x}, \alpha_{y}\right)$,

- Dispersion - only $y$-dispersion exists $\left(\delta_{y}\right)$,

- Derivative of dispersion $\left(d \delta_{y} / d z\right)$.

Of course, these parameters are not independent, since for each coordinate we have

$$
\begin{aligned}
x_{\max }^{2} & =\epsilon \beta \\
\theta_{\max }^{2} & =\frac{\epsilon}{\beta}\left(1+\alpha^{2}\right)
\end{aligned}
$$

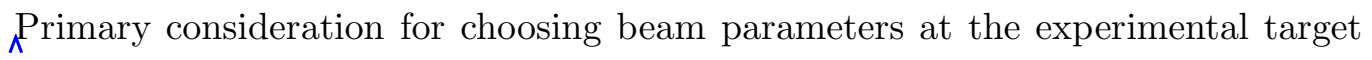
was limiting divergence of the beam to $1 \mathrm{mrad}$, required by beam Cherenkov counters, and spot size of $2.5 \mathrm{~cm}$, the size of the secondary target. Another more subtle factor is that non-dispersive beam lines tend to be more forgiving to errors in quadrupole gradients 


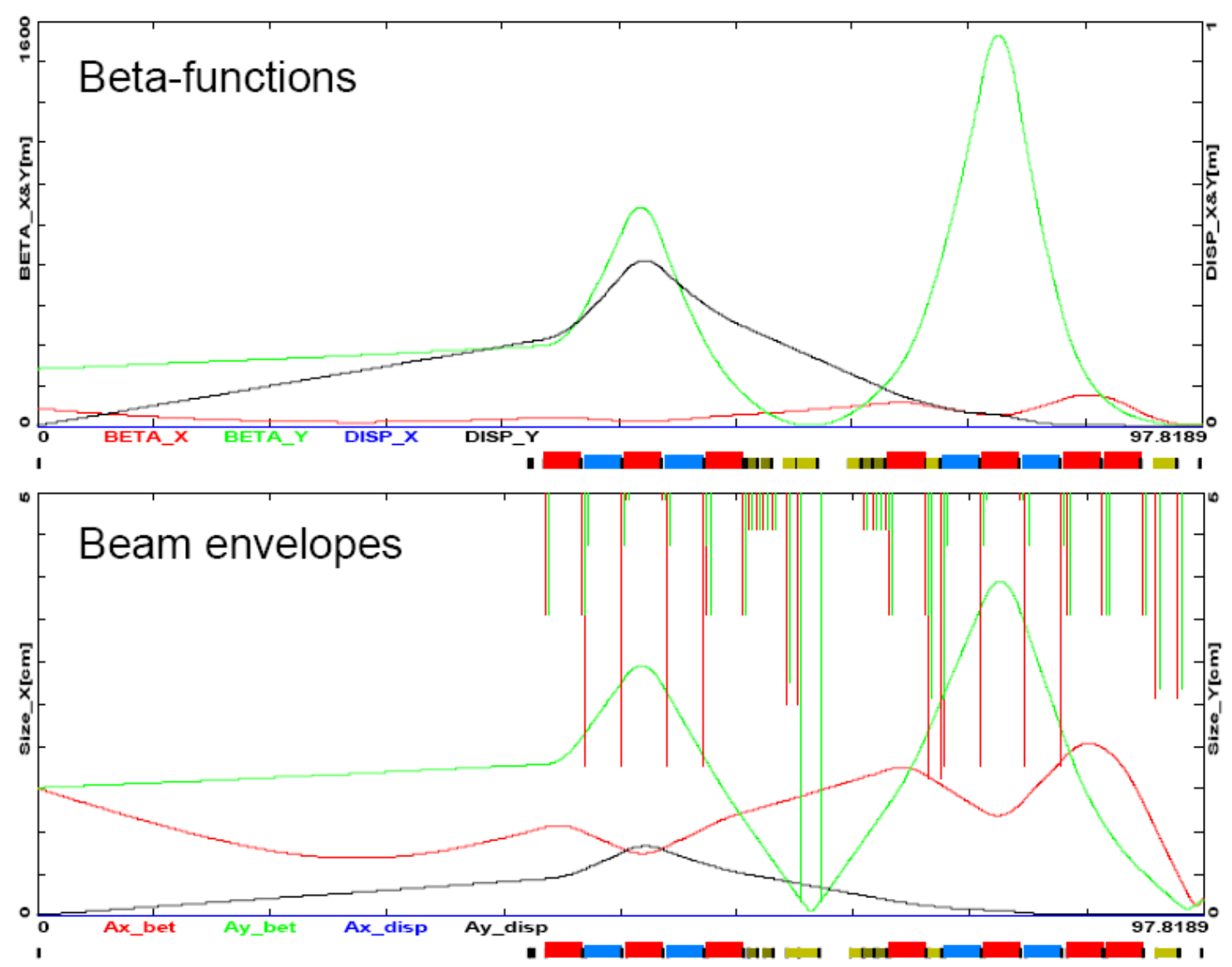

Figure 5.4: Beta functions and beam envelopes for a solution which satisfies the criterion of staying far away from the post-collimator apertures and touching the largest number of apertures upstream of the collimator.

and therefore $\boldsymbol{\Lambda}_{\boldsymbol{1}}$ more stable. Hence dispersion was chosen to be 0 at the experimental target. Of these parameters, we can chose to vary $x_{x / y, \max }, \theta_{x / y, \max }, \alpha_{x / y}$, and $d \delta_{y} / d z$ within reasonable limits and look for current settings to satisfy specified requirements.

An acceptable solution was obtained with $x_{x / y, \max }=1.5 \mathrm{~cm}, \theta_{x, \max }=0.5 \mathrm{mrad}$, $\theta_{y, \max }=0.1 \operatorname{mrad}, \alpha_{x}=2, \alpha_{y}=-0.9$, and $d \delta_{y} / d z=5 \times 10^{-3}$. Figure 5.4 shows beta functions and beam envelopes for this backward beam line. This choice of magnet currents keeps the beam at least $1 \mathrm{~mm}$ away from all beam pipes downstream of the collimator, and has the beam envelope touch the collimator. 


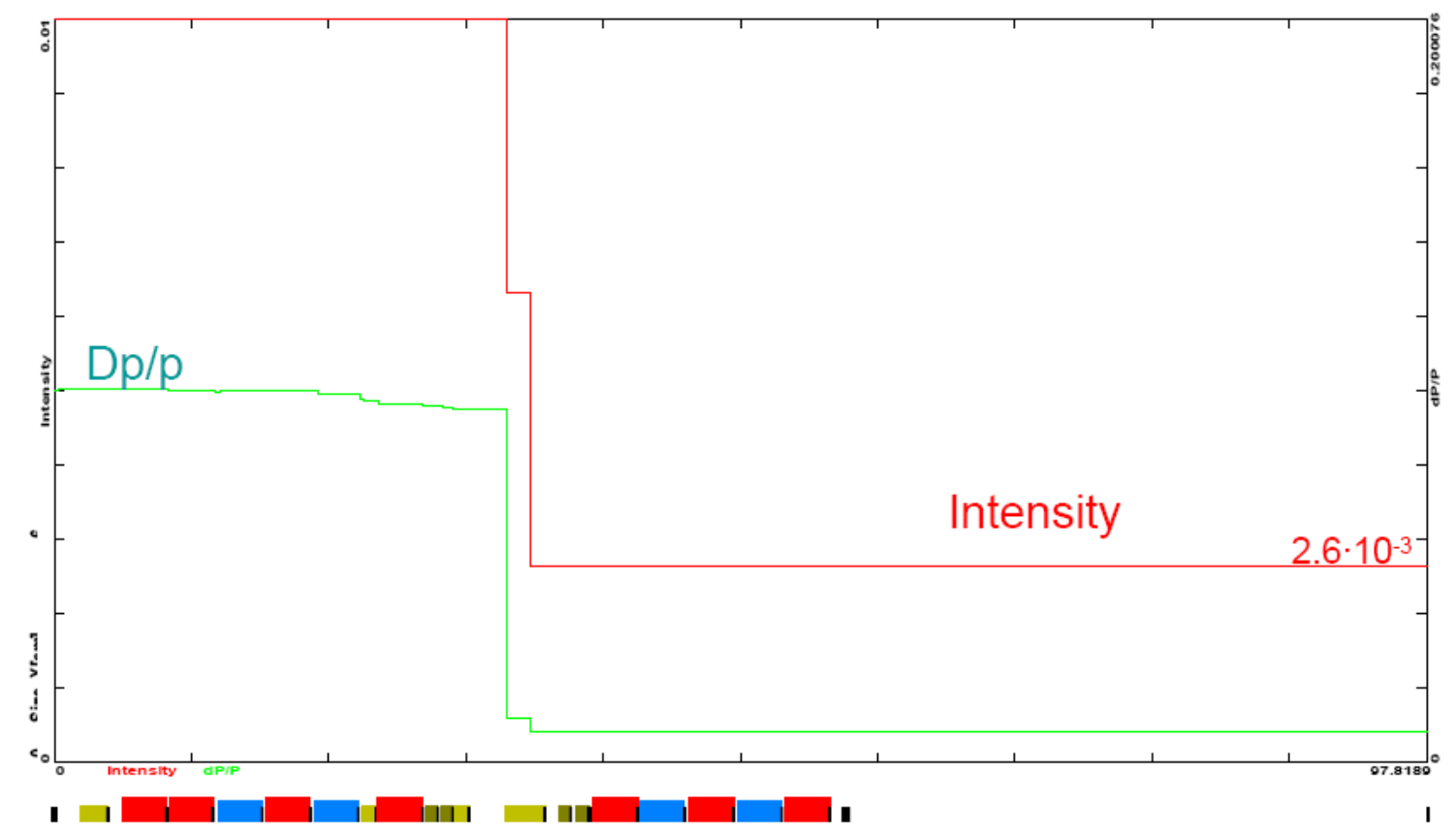

Figure 5.5: Tracking results for beamline settings obtained through reversed beamline. Unlike the first solution, this one does not show scraping in the fourth dipole magnet.

When particles were tracked forward through this solution, not only seraping was greatly reduced, but more particles were getting through, as is clear from Figure 5.5. Clear improvement was observed in the quality of experimental data when quadrupole currents derived from this solution were set, and a somewhat refined solution inspired by this modeling was used to set secondary beam. 


\section{Chapter 6}

\section{Event Reconstruction}

\subsection{Track Fitting}

The overwhelming majority of particle trajectories (tracks) in MIPP are fit either to straight lines or track templates. Approach to both methods is presented in this section.

The task is to fit a 3-dimensional curve to a collection of measurements with different orientations in $x y$-plane (also referred to as views) at different $z$ locations. Defining measurement this way allows one to easily combine 3-dimensional points computed in the TPC with 2-dimensional chamber measurements: one simply splits the TPC point into two 2-dimensional measurements.

Our convention is to measure the angle between wire orientation and $y$-axis, so that $x$-measurements (vertical wires) have $\theta=0$ and $\theta$ is increasing clockwise. To make coordinate $u$ of a given view collinear with $x$ and $y$-axis when appropriate, $\theta=-90^{\circ}$ for $y$-measurements (horizontal wires). Figure 6.1 shows a diagram of this convention. Then a point $(x, y)$ can be converted to $u$ in a given view through

$$
u=x \cos \theta-y \sin \theta
$$

\subsubsection{Straight Line Fitting}

Fitting a straight line to collection of measurements is easily done through generalized least squares method. A line in space is described by 6 parameters. We choose to represent the line through the two points at smallest and largest $z:\left(x_{1}, y_{1}, z_{1}\right)$ and 


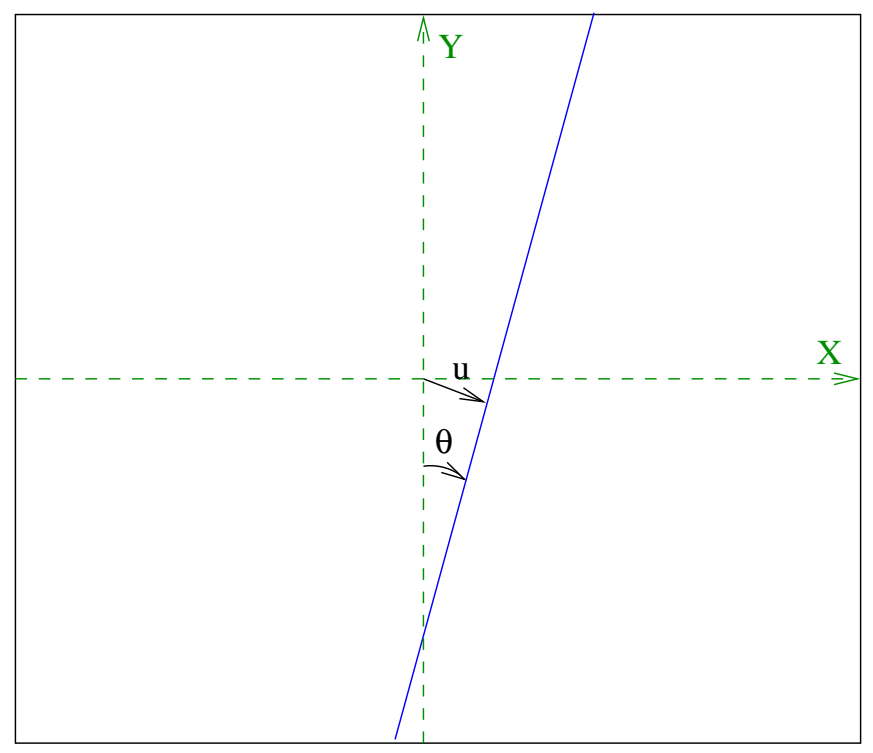

Figure 6.1: Sketch of wire plane geometry.

$\left(x_{2}, y_{2}, z_{2}\right)$. Once the choice of $z_{1}$ and $z_{2}$ is made, we need to determine the four parameters $\vec{p}=\left(x_{1}, y_{1}, x_{2}, y_{2}\right)$.

At any $z$, we have

$$
\left\{\begin{array}{l}
x(z)=\frac{x_{1}\left(z-z_{2}\right)-x_{2}\left(z-z_{1}\right)}{z_{1}-z_{2}} \\
y(z)=\frac{y_{1}\left(z-z_{2}\right)-y_{2}\left(z-z_{1}\right)}{z_{1}-z_{2}}
\end{array}\right.
$$

Then using Equation 6.1 we can predict $u$ in a given view at $z$ through

$$
u(z ; i)=\sum_{j=1,4} c_{i j} p_{j}
$$

where

$$
\vec{c}_{i}=\left(\begin{array}{c}
\frac{z-z_{2}}{z_{1}-z_{2}} \cos \theta_{i} \\
-\frac{z-z_{2}}{z_{1}-z_{2}} \sin \theta_{i} \\
-\frac{z-z_{1}}{z_{1}-z_{2}} \cos \theta_{i} \\
\frac{z-z_{1}}{z_{1}-z_{2}} \sin \theta_{i}
\end{array}\right)
$$

With $N$ measurements, we write the least squares equation

$$
\chi^{2}=\sum_{i=1, N} w_{i}\left(u_{i}-\sum_{j} c_{i j} p_{j}\right)^{2}
$$


where $w_{i}$ is the weight given to the wire in the track. We seek for solution which minimizes $\chi^{2}$, that is

$$
\frac{\partial}{\partial p_{k}} \chi^{2}=2 \sum_{N} w_{i} c_{i k}\left(\sum_{j} c_{i j} p_{j}-u_{i}\right)=0
$$

We can rewrite Equation 6.5 as a matrix equation

$$
\vec{v}=\mathbf{M} \vec{p}
$$

where

$$
\begin{aligned}
v_{j} & =\sum_{i} w_{i} u_{i} c_{i j} \\
M_{j k} & =\sum_{i} w_{i} c_{i j} c_{i k}
\end{aligned}
$$

Solving Equation 6.5 gives

$$
\begin{aligned}
\vec{p} & =\mathbf{M}^{-1} \vec{v}, \\
\chi^{2} & =\sum_{i} u_{i} w_{i}^{2}-\vec{p} \cdot \vec{v} .
\end{aligned}
$$

Finally, since

$$
\frac{1}{2} \frac{\partial^{2}}{\partial p_{i} \partial p_{j}} \chi^{2}=M_{i j}
$$

i.e. $M$ is the inverse covariance matrix, errors on parameters are given by

$$
\sigma_{p_{i}}^{2}=M_{i \dot{j}_{k}}^{-1}
$$

\subsubsection{Template Track Fitting}

When track traverses regions of space with significant magnetic field, one needs to determine track momentum in addition to location and direction at a given $z_{0}$. If one has a rough guess at track parameters and the field is sufficiently smooth, the problem can be solved by fitting the set of measurements to a track template. Advantage of this method is the small required number of swims through the magnetic field. Disadvantage of this method is that track direction is represented by $d x / d z$ and $d y / d z$, which are difficult to use when track angle to $z$-axis is large.

As charged particle traverses magnetic field, to first order its bend angle and therefore displacement from ${ }_{\wedge}$ straight-line track is proportional to $q / p$, the charge and momentum 
of the particle. That is, at a given $z$, we can represent particle position with 5 parameters: $Q=q / p$, position $(x, y)$ and direction $(d x / d z, d y / d z)$ at $z=z_{0}$ :

$$
\left\{\begin{array}{l}
x=x_{0}+\left.\frac{d x}{d z}\right|_{z=z_{0}} \cdot\left(z-z_{0}\right)+Q \lambda_{x}(z) \\
y=y_{0}+\left.\frac{d y}{d z}\right|_{z=z_{0}} \cdot\left(z-z_{0}\right)+Q \lambda_{y}(z)
\end{array}\right.
$$

where $\lambda_{x}$ and $\lambda_{y}$ can be interpreted as displacement from straight-line trajectory for a particle with $q / p=1$ and can be calculated by swimming a track with guess parameters $(Q \neq 0)$ from $z_{0}$ and setting

$$
\left\{\begin{array}{l}
\lambda_{x}(z)=\frac{1}{Q}\left(x_{s w i m}-x_{0}-\left.\frac{d x}{d z}\right|_{z=z_{0}} \cdot\left(z-z_{0}\right)\right) \\
\lambda_{y}(z)=\frac{1}{Q}\left(y_{\text {swim }}-y_{0}-\left.\frac{d y}{d z}\right|_{z=z_{0}} \cdot\left(z-z_{0}\right)\right) .
\end{array}\right.
$$

When $|d x / d z|<1$ and $|d y / d z|<1$ at $z_{0}$ and $z$, the errors from this representation are negligible.

Equipped with Equations 6.7 and 6.8, we need a guess at track parameters to compute $\lambda x, y$ at $z$ of every measurement, and we can reuse linearized chi squared formalism with

$$
\vec{p}=\left(\begin{array}{c}
x\left(z_{0}\right) \\
y\left(z_{0}\right) \\
d x / d z\left(z_{0}\right) \\
d y / d z\left(z_{0}\right) \\
Q\left(z_{0}\right)
\end{array}\right), \quad \overrightarrow{c_{i}}=\left(\begin{array}{c}
\cos \theta_{i} \\
-\sin \theta_{i} \\
\left(z_{i}-z_{0}\right) \cos \theta_{i} \\
-\left(z_{i}-z_{0}\right) \sin \theta_{i} \\
\lambda_{y}\left(z_{i}\right) \cos \theta_{i}-\lambda_{x}\left(z_{i}\right) \sin \theta_{i}
\end{array}\right)
$$

Notice that if the track template takes energy loss into account, this approach will to first order ${ }_{\Lambda}$ behave correctly, especially if energy loss over the length of the track is small compared to initial track energy.

\subsubsection{Fail Safe Fitting}

In case template track fit is inadequate for a track, the fail-safe method of fitting

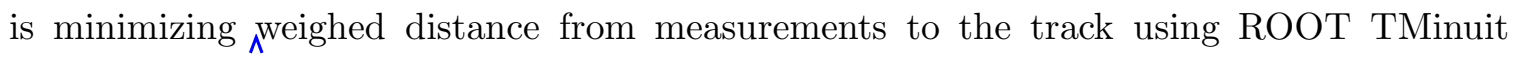
package. Different weights can be chosen for $x, y, z$, so that even when the track is nearly perpendicular to $z$-axis its parameters will be computed correctly. 
Given point $(x, y, z)$ the closest point on the track $\left(x_{t}, y_{t}, z_{t}\right)$ was found by minimizing

$$
D^{2}=w_{x}\left(x-x_{t}\right)^{2}+w_{y}\left(y-y_{t}\right)^{2}+w_{z}\left(z-z_{t}\right)^{2} .
$$

If the track is straight, from the closest point on the track stored in memory, the step that one needs to take to minimize $D^{2}$ is given by ${ }^{1}$

$$
s=\frac{w_{x}\left(x-x_{t}\right) p_{x}+w_{y}\left(y-y_{t}\right) p_{y}+w_{z}\left(z-z_{t}\right) p_{z}}{w_{x} p_{x}^{2}+w_{y} p_{y}^{2}+w_{z} p_{z}^{2}},
$$

where unit vector $\left(p_{x}, p_{y}, p_{z}\right)$ represents direction of the track at $\left(x_{t}, y_{t}, z_{t}\right)$. If the track is not a straight line, then one has to repeat the procedure until $s$ is sufficiently small.

\subsection{Wire Chambers}

MIPP wire chambers are grouped into 3 triplets (BC123, DC123, DC4/PWC56) with two magnets separating the triplets. To first order, curvature due to magnetic field is negligible between the magnets as well as before Jolly Green Giant and after Rosie, so that one can reconstruct a straight line segment in each group. Once segments are found, it is simple to test whether a combination of ${ }_{\wedge} \mathrm{DC} 123$ segment with ${ }_{\wedge} \mathrm{DC} 4 / \mathrm{PWC} 56$ segment appears to form a reasonable track candidate bent by Rosie. Finally, if track candidate momentum is similar to beam momentum, one can check whether combining the candidate with ${ }_{\wedge} \mathrm{BC} 123$ segment will give a reasonable 9-chamber track.

Having reliable track fits with all 9 chambers was crucial to aligning $\boldsymbol{\Lambda}_{\boldsymbol{\Lambda}}$ hambers and finding inconsistencies of $\boldsymbol{\Lambda}^{\text {description of }} \boldsymbol{\wedge}$ spectrometer geometry.

\subsubsection{Wire Clusters}

A wire cluster is defined as either one wire or a group of contiguous wires. In drift chambers, we clustered wires only if their hit times were similar and the cluster size is

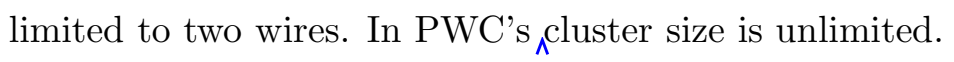

Chamber preamplifiers and discriminators would occasionally ring so that all 8 wires were hit. In those cases, wires from that preamp are not used for tracking in order to improve the signal to noisg ratio.

\footnotetext{
${ }^{1}$ If $w_{x}=w_{y}=w_{z}=1$ Equation 6.11 reduces to dot product of track direction and vector pointing from $\left(x_{t}, y_{t}, z_{t}\right)$ to $(x, y, z)$.
} 
Once wires were grouped, the geometric center of the cluster in chamber coordinate system is computed through

$$
u_{l o c}=D_{\text {plane }}\left(W-W_{0}\right),
$$

where $D_{\text {plane }}$ is the wire spacing, and $W_{0}$ is the hypothetical wire that goes through $u=0$ in local reference frame. A point on the wire $\left(u \cos \theta_{l o c},-u \sin \theta_{l o c}, z_{l o c}\right)$ is then transformed to global reference frame $\left(x_{\text {glob }}, y_{\text {glob }}, z_{\text {glob }}\right)$, taking into account chamber rotation and position. Finally, the global $u$ of the cluster is obtained from $\left(x_{g l o b}, y_{g l o b}\right)$ through Equation 6.1. Note that at this stage $z_{g l o b}$ is not a meaningful coordinate because we do not know where along the cluster the hit occurred.

\subsubsection{Wire Crosses}

To simplify track segment pattern recognition, we formed 3-dimensional space points from wires in at least 2 views of the same chamber. Having 3D space points enabled us to apply the same track finding algorithm to $\mathrm{BC} 1+\mathrm{BC} 2+\mathrm{BC} 3, \mathrm{DC} 1+\mathrm{DC} 2+\mathrm{DC} 3$, and DC4+PWC5+PWC6 segments. Wire plane angles (see Table 2.1) are the same for BCs and DCs - subject to small corrections from alignment - so one could imagine doing 2dimensional $u z$-search in BCs and DCs, but having one algorithm for all triplets of chambers made it easier to maintain code.

From Equation 6.1, it follows that coordinates of intersection of two wires is given by

$$
\left\{\begin{array}{l}
x=\frac{u_{1} \sin \theta_{2}-u_{2} \sin \theta_{1}}{\cos \theta_{1} \sin \theta_{2}-\sin \theta_{1} \cos \theta_{2}} \\
y=\frac{u_{1} \cos \theta_{2}-u_{2} \cos \theta_{1}}{\cos \theta_{1} \sin \theta_{2}-\sin \theta_{1} \cos \theta_{2}}
\end{array}\right.
$$

If more than two wires belong to a cross, its position can be computed through least squares approach (Equation 6.4), where

$$
\vec{p}=\left(\begin{array}{l}
x \\
y
\end{array}\right), \quad \vec{c}_{i}=\left(\begin{array}{c}
\cos \theta_{i} \\
-\sin \theta_{i}
\end{array}\right) .
$$

Wire crosses are then found with the following algorithm:

1. Calculate intersection of two wire clusters from different planes which do not belong to a common wire cross and verify that $(x, y)$ is within the active chamber volume.

2. Look for wire clusters consistent with calculated $(x, y)$ in remaining two views of the chamber. 


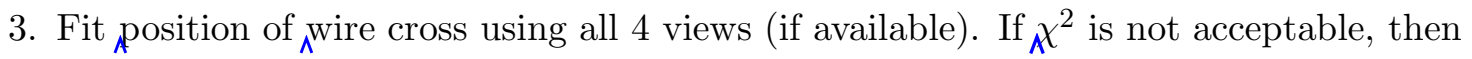
choose the 3 -plane combination with the better $\chi^{2}$.

Once all crosses are found, crosses which are subset of a different cross are eliminated.

The final step in the algorithm is to compute the number of clusters in each cross that belong to crosses with more clusters. Crosses where all wires are used in larger crosses are typically shadows of valid crosses, and they were not used to seed track segments.

\subsubsection{Track Segments}

Track segment finding is similar to wire cross finding: the algorithm evaluated all possible combinations of chamber space points and kept the ones that contained information from all 3 chambers in a triplet and had acceptable $\chi^{2}$.

For the purpose of segment finding, a data structure was defined, which the following information:

- Wire crosses in the segment,

- Wire clusters in the segment,

- Minimum and maximum wire time,

- Segment fit parameters as $\left(x_{1}, y_{1}, z_{1}\right)$ and $\left(x_{2}, y_{2}, z_{2}\right)$.

A cross/cluster data structure was defined to hold

- Minimum and maximum wire time for cross/cluster,

- List of segments the cross/cluster is in.

With data organized this way, any of the following tests become fast:

- Are two crosses compatible in time?

- Is cross/cluster compatible in time with existing segment?

- Are two crosses already part of one segment?

- How many clusters are shared between two segments?

${ }_{\Lambda}$ Track finding algorithm follows these steps: 
1. Loop over all binary combinations of crosses of every pair of chambers.

2. If the two crosses compatible in time and are not part of a formed segment, continue to next step.

3. Fit a 3-dimensional line to the clusters of the two crosses and make prediction of position at the third chamber.

4. Given prediction, find the closest cross or cluster which is compatible in time with

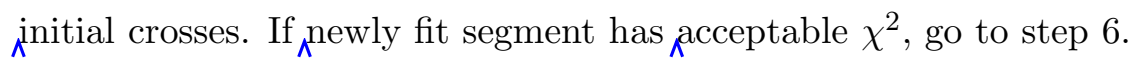

5. Find the closest wire cluster in each view of the third chamber compatible in time

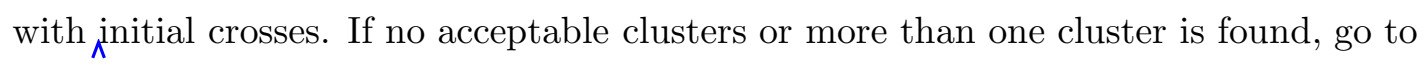
step 1 .

6. If the segment has the minimum required number of clusters, save it and update track list of every cross/cluster.

At the end of segment search, it was important to filter out essentially duplicate tracks. Any track that had less than required number of unique clusters was not saved.

\subsubsection{Chamber Track Candidates}

A chamber track candidate is defined as a combination of DC123 and DC4/PWC56 track segments. ${ }_{\wedge}$ Track bend in the Rosie magnet enables us to measure momentum of the particle. Search for track candidates was done by matching predicted position in the center of Rosie from both segments and matching $d y / d z$ of the two segments. While the windows on both selection ${ }_{\wedge}$ were quite wide $(12 \mathrm{~cm}$ match in $x, 10 \mathrm{~cm}$ in $y$, and $5 \mathrm{mrad}$ in $d x / d z)$, they helped reduce the combinatorics of possible track candidates before a template track fit was attempted.

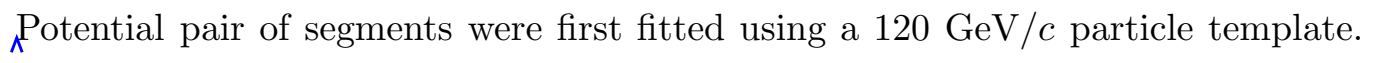
The results of the fit were used to obtain a better template and the track was refit. The track was accepted or rejected based on the $\chi^{2}$ of the second fit.

${ }_{\Lambda}$ Final step of the algorithm was to attempt to match the track with ${ }_{\Lambda}^{\mathrm{BC} 123}$ track segment. Obviously wrong candidates were filtered out by requiring that the charge of the candidate match the charge of beam particles in that run, and that $\wedge_{\wedge}$, 

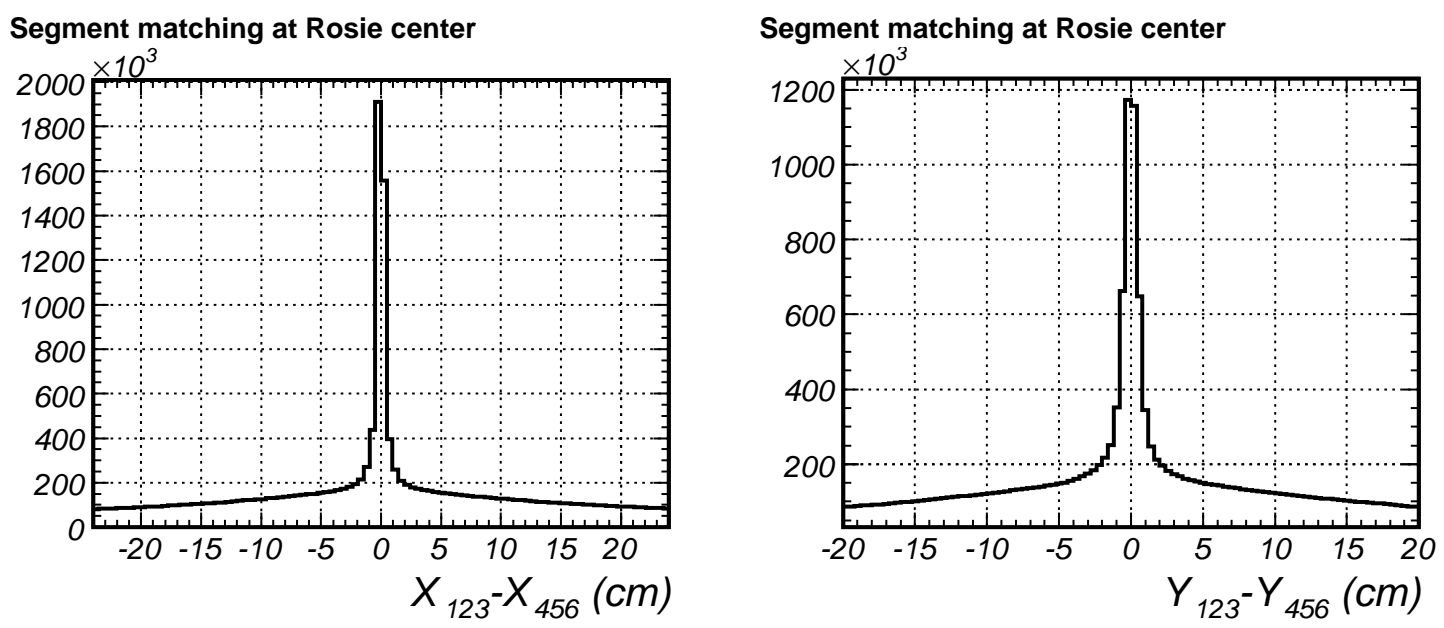

Figure 6.2: Matching of DC123 and DC4/PWC56 segments in the center of Rosie.
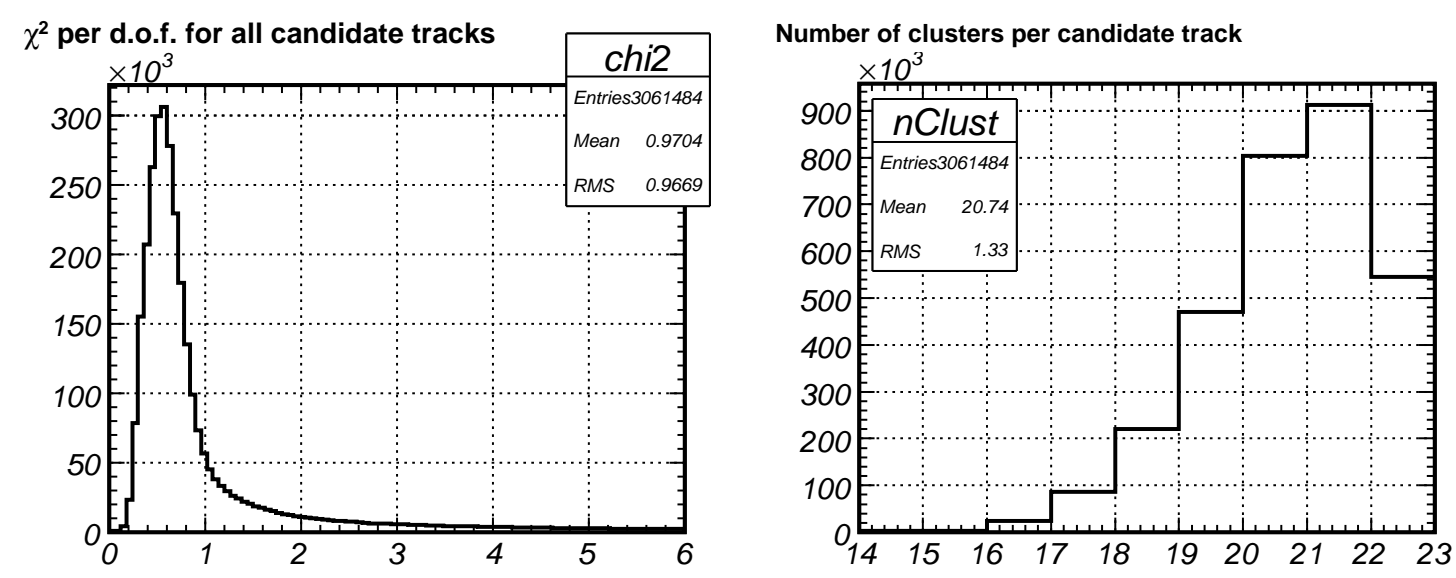

Figure 6.3: Distribution of $\chi^{2}$ per degree of freedom and number of clusters in chamber track candidates. 
0.8 of beam particle momentum. Decision on whether to keep the track candidate as a 6 -chamber or a 9-chamber object was made based on the $\chi^{2}$ of the resulting track.

\subsection{Time Projection Chamber}

The TPC dominates event size and is the most complex detector to reconstruct. The primary problems facing track reconstruction in the TPC are

- Large dead sections of the detector;

- $16 \mu$ s integration time, which makes the TPC very sensitive to out-of-time tracks.

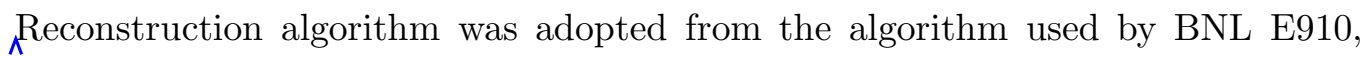
which is described in great detail in [43] and MIPP-specific aspects in [36]. The main steps of reconstruction are:

1. Create two-dimensional contiguous clusters in each padrow $(z$-slice) of the TPC. Each cluster is fitted to a Gamma function in $t: q(t)=A t^{\lambda} e^{-t}$, and a Gaussian in $x$. For hits with insufficient number of pad columns, the position in $x$ was calculated through weighted mean, using charge collected on the pad as the weight.

2. Compute hit position by computing electron trajectory starting from the TPC anode plane (see Section 7.5.2).

3. Search for tracks using what amounts to a simplified Kalman filter approach. Starting from the downstream end of the chamber - where the distance between tracks is the largest - a track is formed by connecting the closest hits in the neighboring padrows. The track is then extended and position prediction is made in the next padrow. Acceptable hits are added into the track until no new hits are found in the upstream direction. ${ }_{\wedge}$ Hit search is then reversed to pick up hits in the downstream direction.

4. Similar tracks - those that share a large fraction of their hits - are merged to remove essentially duplicated tracks. 

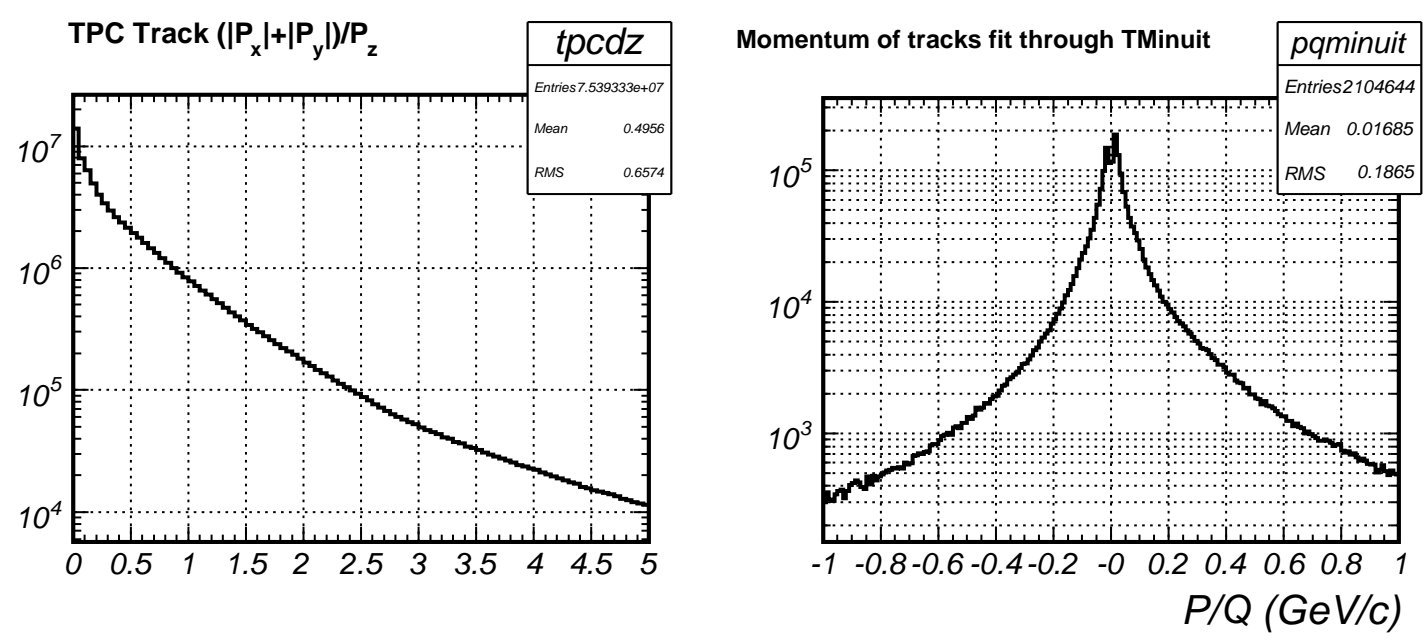

Figure 6.4: Ratio for selecting tracks which are to be fit through TMinuit and momenta of those tracks.

\subsection{Global Tracking}

The objectives of global tracking are

1. ${ }_{\wedge}^{\text {Refit TPC tracks using real JGG magnetic field; }}$

2. ${ }_{\Lambda}$ Combine TPC tracks with chamber track candidates, track segments, or stand-alone wire clusters.

\subsubsection{Refitting TPC tracks}

If the track angle is not too large with respect to the $z$-axis, template track fit approach is adequate to refit the TPC track. However, if the track angle to $z$-axis is too large, $\lambda_{\Lambda}$ template fitter is not able to handle it adequately, and the track is passed to $\hat{\lambda}_{\boldsymbol{1}}$ fail-safe TMinuit fitter. Selection of the fitter was based on two criteria:

1. Ratio $\left(\left|P_{x}\right|+\left|P_{y}\right|\right) / P_{z}$ in the middle of the track, at $\frac{1}{4}$ and $\frac{3}{4}$ of $z$ along the track;

2. Number of padrows in the track.

Tracks with ratio above 4 and tracks that span fewer than 4 padrows, were not attempted to be fit through template fitter. The tracks selected for slow fitter are predominantly low momentum tracks (see Figure 6.4). 
In either fitter, track parameters were evaluated in the middle of the track. This way potential problems with very large $d x / d z$ or $d y / d z$ at the pivot point are minimized.

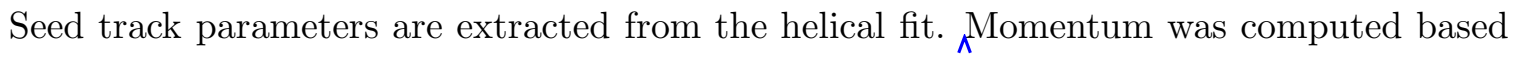
on track curvature, under assumption of homogeneous magnetic field.

In the event that the template fitter could not re-swim the track from the first hit to the last hit, ${ }_{\Lambda}$ template fitting was considered failed, and the track was passed to the slower TMinuit fitter.

\subsubsection{Cleaning up TPC Tracks}

$\aleph^{\mathrm{MIPP}}$ TPC reconstruction algorithm had two minor problems which resulted in

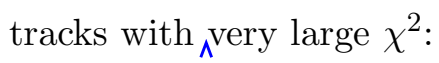

- Two nearby tracks could be merged together;

- ${ }_{\Lambda}$ Track finding algorithm could grab hits that were inconsistent with the rest of the hits on the track.

To alleviate these problems, we took the following approach. Hits were removed from the track until its $\chi^{2}$ per degree of freedom was under 2 . Then we continued to remove those hits which contributed more than 8 times than the average contribution to $\chi^{2}$. This approach cleaned up TPC tracks without sacrificing well reconstructed tracks.

\subsubsection{Merging Tracks}

Prior to fitting TPC tracks using the magnet field map, tracks were merged using a more sophisticated approach than just looking at the number of shared TPC and wire chamber hits. Similarity of tracks was judged by computing "cross $\chi^{2}$ " as the sum of weighted residuals squared of one track using parameters of another track. If the goodness of fit based on cross $\chi^{2}$ was larger than 0.1 or the ratio of the first track's $\chi^{2}$ to cross $\chi^{2}$ was larger than 0.15 , then the tracks were merged together.

\subsubsection{Matching TPC Tracks to Chambers}

Global tracks - those including TPC and chamber information - are formed by first attempting to match TPC tracks to chamber track candidates at DC1. If a matching candidate is not found, then a matching DC123 segment is searched for. If the segment is 
TPC/TrkCand x-matching at DC1

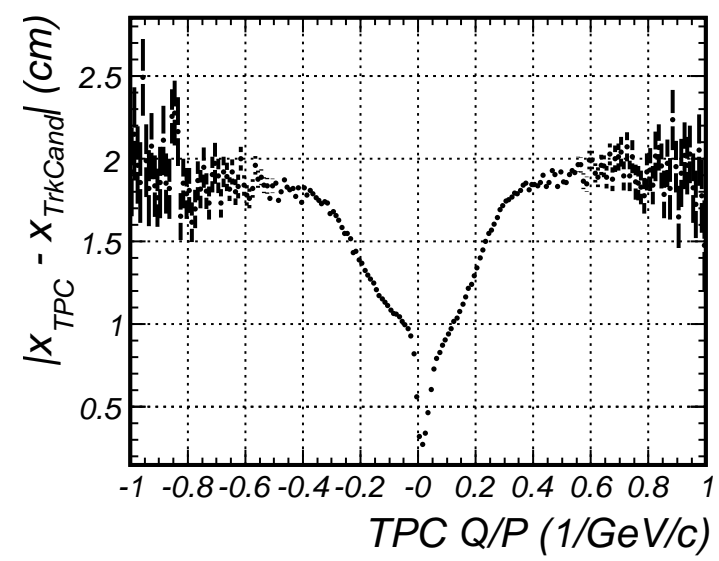

TPC/TrkCand y-matching at DC1

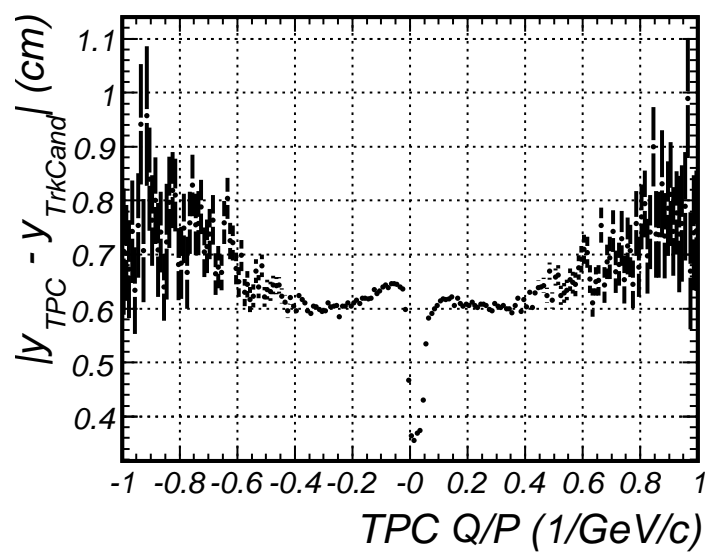

Figure 6.5: Dependence of position matching of the TPC and chamber track candidates when position is computed at DC1.

not found, then we search for nearest wire clusters in DC1, DC2, and DC3, refitting the track with the new information each time a hit is found. If predicted track position at a chamber is outside of the active area of the chamber, then no attempt is made to extend the track further. The tracks that were extended to DC3, the process continues in downstream chambers, first searching for matching C456 track segments and descending to the level of clusters if it is necessary.

\subsubsection{Tracks in the Event Stream}

Saving only track measurements and parameters to event stream has the potential of slowing down analysis modules that need tracks to predict track position and direction at various $z$ along the spectrometer, since every module would have to re-swim the track

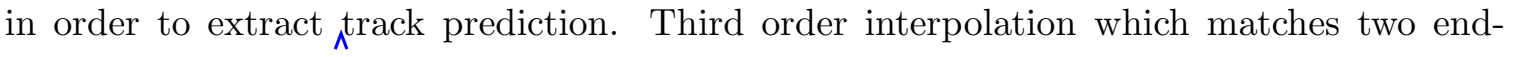
points and track direction at the two points was implemented to make trajectory prediction without swimming. While global tracking module still had the states of the swum track, points were inserted in the middle of two neighboring points so that third order prediction at $1 / 4,1 / 2$, and $3 / 4$ distance in $z$ was within 100 micron of the swim prediction. This accuracy was sufficient for all modules which used tracking results. 

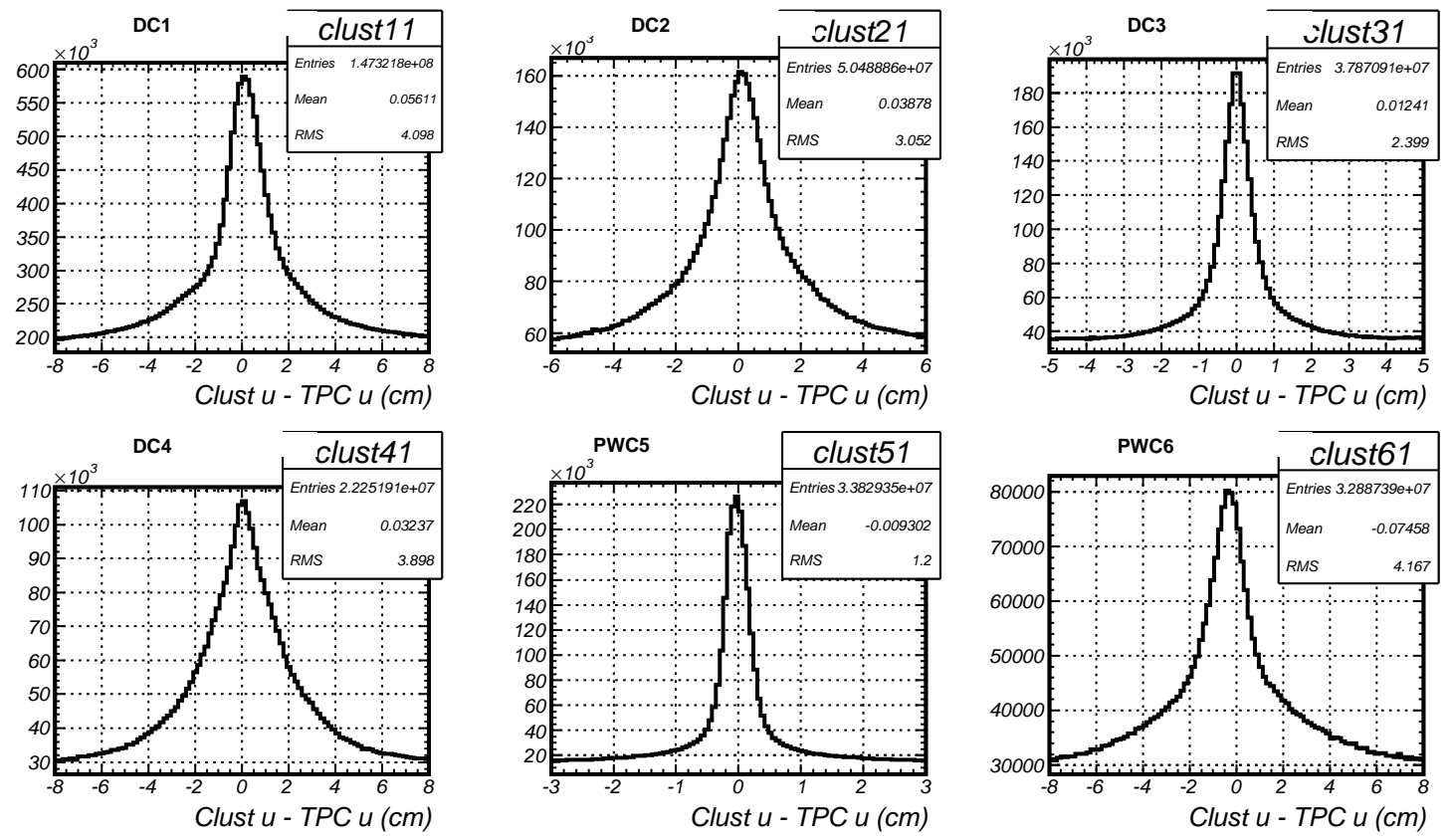

Figure 6.6: Matching of tracks at the six chambers. Notice that the $x$-axis scales are different.

\subsection{Vertex Reconstruction}

Identifying primary and decay vertices is essential for data analysis, as not only one can determine where the track came from, but also track parameters from a vertex constrained fit are more trustworthy ${ }_{\wedge \wedge} \mathrm{MIPP}$ vertex reconstruction was split into two parts: vertex finding and vertex constrained track fitting.

\subsubsection{Vertex Finding}

One of the difficulties in finding vertices is the fact that beam conditions were not always clean. The algorithm that worked very well, is based on deterministic annealing filter (DAF).

Using TMinuit, we can fit for the point which minimizes the weighted distance squared to all tracks in the event (See Equation 6.10). The filter helps select the tracks associated with the vertex by reducing the weight of the outliers with

$$
w_{D A F}=\frac{1}{1+\exp \left(\frac{D_{i}^{2}-R_{c}^{2}}{2 T_{D A F}}\right)},
$$




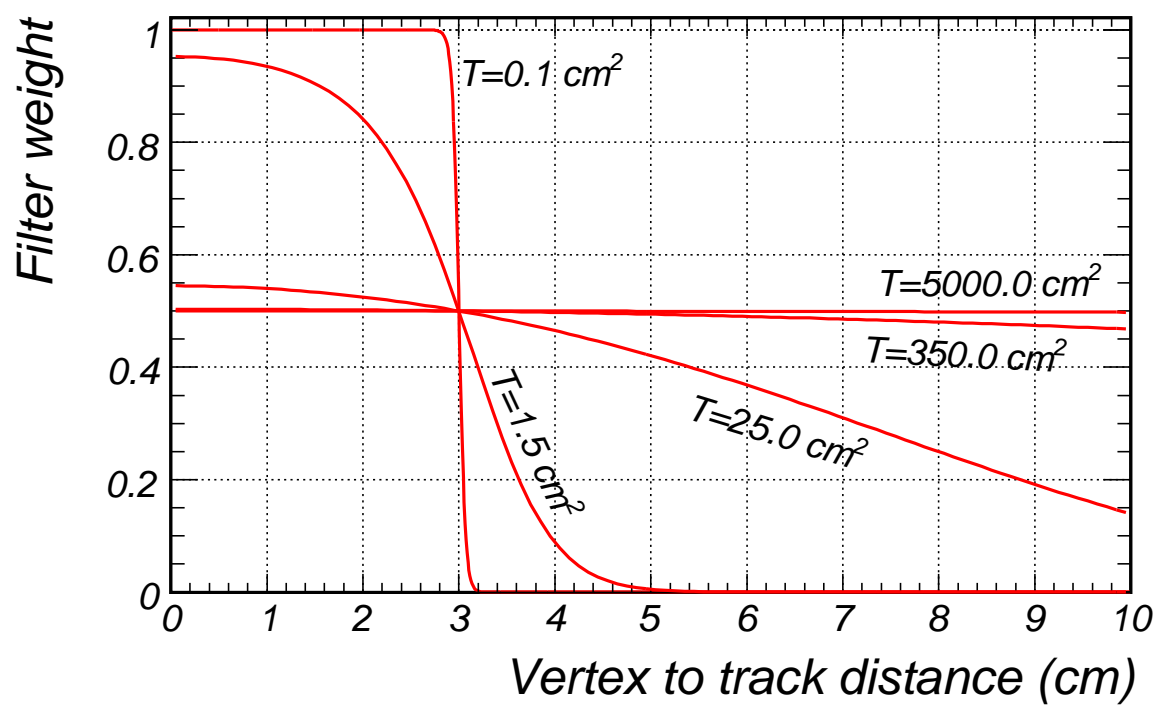

Figure 6.7: Shown are ${ }_{\mu} \mathrm{DA}$ filter weight function for the temperatures used at MIPP.

where $D_{i}$ is the distance to the track given vertex position, $D_{c}$ is the filter critical radius, and $T_{D A F}$ is the temperature. As the temperature is decreased, the weight of the outliers is reduced and the weight $t_{\Lambda}$ of the tracks with $D_{i}^{2}<D_{c}^{2}$ is not affected. Figure 6.7 shows the weight function for the temperature schedule used by our vertex finder.

When the temperature is reduced sufficiently slowly, the filter converges on the most significant vertex even if multiple vertices exist in the event. The tracks that were included into the vertex are excluded from the available track list and the process is repeated.

In the events where the only two tracks are incoming beam track measured by the beam chambers and the same track measured by TPC and drift chambers, we found that when the temperature was lowered to $25 \mathrm{~cm}^{2}$, it was necessary to repeat the procedure at the same temperature so that the fitted vertex position would prefer to land in the middle of the two tracks rather than exclude one of them completely.

\subsubsection{Vertex Constrained Fitting}

Fitting a vertex with $N$ particles involves determining $3(N+1)$ parameters: ${ }_{\Lambda}$ vertex position $\left(x_{v}, y_{v}, z_{v}\right)$, and track direction $\lambda_{\Lambda}$ and momenta $\left(d x_{i} / d z, d y_{i} / d z ; q / p_{i}\right)$. Template track fitting can be easily linearized for all parameters except $z_{v}$ if the template coefficients are fairly constant as a function of $x_{v}$ and $y_{v}$. Following ${ }_{\wedge}$ conventions set in Equations 6.1 
and 6.7, we now write vertex $\chi_{v}^{2}$ as

$$
\begin{aligned}
\chi_{v t x}^{2}=\sum_{i=1}^{N} \sum_{j=1}^{n_{i}} w_{i j} & \left\{\left[x_{v}+\frac{d x_{i}}{d z}\left(z_{i j}-z_{v}\right)+Q_{i} \lambda_{x, i}\left(z_{i j}\right)\right] \cos \theta_{i j}\right. \\
& \left.-\left[y_{v}+\frac{d y_{i}}{d z}\left(z_{i j}-z_{v}\right)+Q_{i} \lambda_{y, i}\left(z_{i j}\right)\right] \sin \theta_{i j}-u_{i j}\right\}^{2} .
\end{aligned}
$$

Here $N$ is the total number of tracks in the vertex and $n_{i}$ is the number of measurements

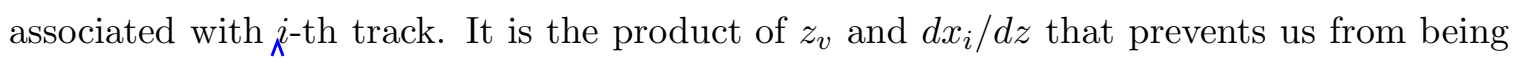
able to linearize the fit for $z_{v}$. With $z_{v}$ held fixed, our solution must satisfy:

$$
0=\frac{\partial}{\partial p_{i}} \chi_{v t x}^{2}=\mathbf{M} \vec{p}-\vec{v}
$$

If we define a vector of constants, likg in Equation 6.9

$$
\vec{c}_{i j}=\left(\begin{array}{c}
\cos \theta_{i j} \\
-\sin \theta_{i j} \\
\left(z_{i j}-z_{v}\right) \cos \theta_{i j} \\
-\left(z_{i j}-z_{v}\right) \cos \theta_{i j} \\
\lambda_{x, i}\left(z_{i j}\right) \cos \theta_{i j}-\lambda_{y, i}\left(z_{i j}\right) \sin \theta_{i j}-
\end{array}\right)
$$

then

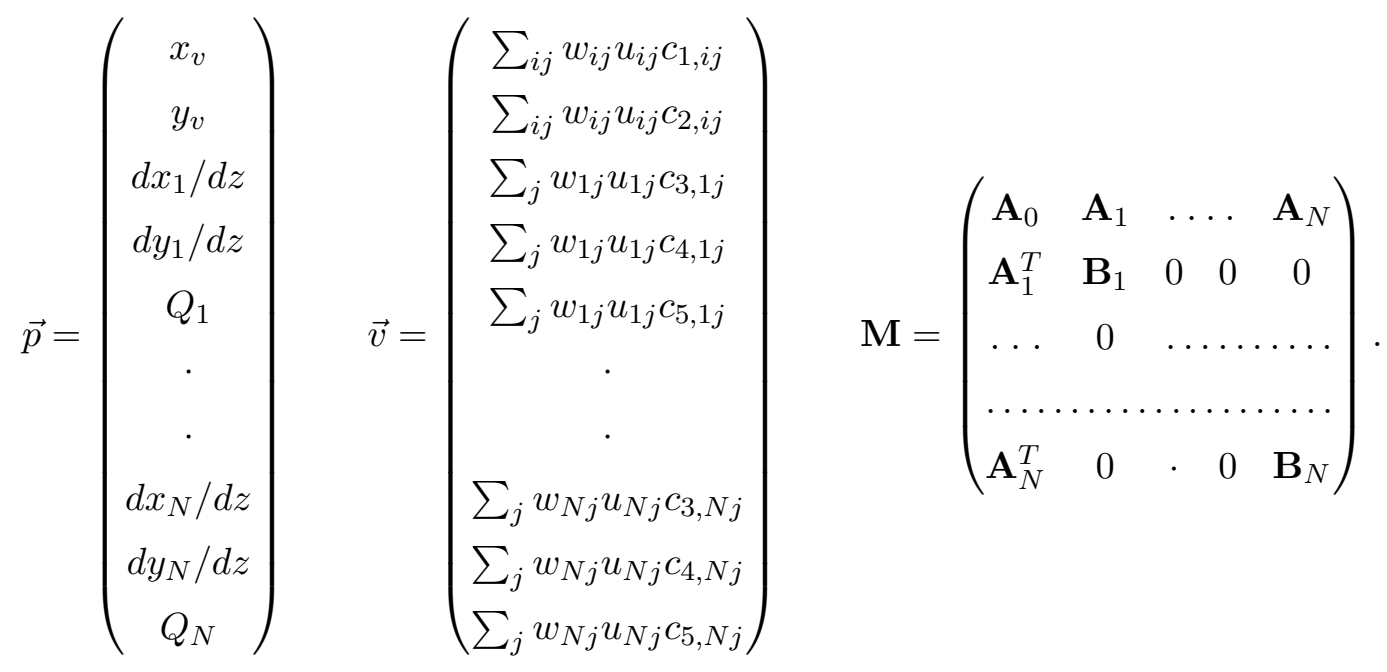


Symmetric matrix $\mathbf{M}$ is made up of the following matrices:

$$
\begin{aligned}
\mathbf{A}_{0} & =\left(\begin{array}{cc}
\sum_{i j} w_{i j} c_{1, i j}^{2} & \sum_{i j} w_{i j} c_{1, i j} c_{2, i j} \\
\sum_{i j} w_{i j} c_{1, i j} c_{2, i j} & \sum_{i j} w_{i j} c_{2, i j}^{2}
\end{array}\right), \\
\mathbf{A}_{i} & =\left(\begin{array}{lll}
\sum_{j} w_{i j} c_{1, i j} c_{3, i j} & \sum_{j} w_{i j} c_{1, i j} c_{4, i j} & \sum_{j} w_{i j} c_{1, i j} c_{5, i j} \\
\sum_{j} w_{i j} c_{2, i j} c_{3, i j} & \sum_{j} w_{i j} c_{2, i j} c_{4, i j} & \sum_{j} w_{i j} c_{2, i j} c_{5, i j}
\end{array}\right), \\
\mathbf{B}_{i} & =\left(\begin{array}{lll}
\sum_{j} w_{i j} c_{3, i j} c_{3, i j} & \sum_{j} w_{i j} c_{3, i j} c_{4, i j} & \sum_{j} w_{i j} c_{3, i j} c_{5, i j} \\
\sum_{j} w_{i j} c_{4, i j} c_{3, i j} & \sum_{j} w_{i j} c_{4, i j} c_{4, i j} & \sum_{j} w_{i j} c_{4, i j} c_{5, i j} \\
\sum_{j} w_{i j} c_{5, i j} c_{3, i j} & \sum_{j} w_{i j} c_{5, i j} c_{4, i j} & \sum_{j} w_{i j} c_{5, i j} c_{5, i j}
\end{array}\right),
\end{aligned}
$$

and $\mathbf{A}_{i}^{T}$ is the transpose of $\mathbf{A}_{i}$.

Thus, the solution to vertex position is given by

$$
\vec{p}=\mathbf{M}^{-1} \vec{v}
$$

In order to determine $z_{v}$, we scan around the seed value of the vertex, and compute $z_{v}$ and error on $z_{v}$ from parabolic fit to the 5 points around the minimum. In practice, since the track template coefficients do change with vertex location, until the computed minimum is close enough to the $z$-coordinate where template coefficients were evaluated, we continue the scan with template coefficients re-evaluated at the new minimum. Most vertex $z$ positions returned by ${ }_{\Lambda} \mathrm{DA}$ filter vertex finder are sufficiently good; so that only one scan is required

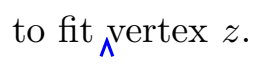

\subsubsection{Fixing Track Momentum in ${ }_{\wedge}$ Vertex Fit}

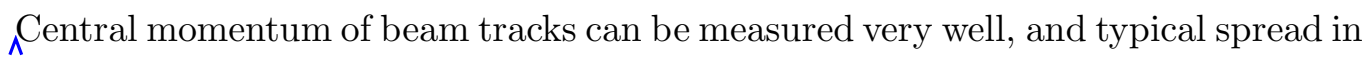
momentum is a few percent, therefore in vertex-constrained fits, momentum of beam tracks should not be varied $\boldsymbol{\wedge}_{\boldsymbol{\wedge}}$ The algorithm outlined above is easily modified to fix momentum of one ore more tracks by changing matrices $\mathbf{A}_{i}$ and $\mathbf{B}_{i}$ to

$$
\begin{aligned}
\mathbf{A}_{i} & =\left(\begin{array}{ll}
\sum_{j} w_{i j} c_{1, i j} c_{3, i j} & \sum_{j} w_{i j} c_{1, i j} c_{4, i j} \\
\sum_{j} w_{i j} c_{2, i j} c_{3, i j} & \sum_{j} w_{i j} c_{2, i j} c_{4, i j}
\end{array}\right), \\
\mathbf{B}_{i} & =\left(\begin{array}{ll}
\sum_{j} w_{i j} c_{3, i j} c_{3, i j} & \sum_{j} w_{i j} c_{3, i j} c_{4, i j} \\
\sum_{j} w_{i j} c_{4, i j} c_{3, i j} & \sum_{j} w_{i j} c_{4, i j} c_{4, i j}
\end{array}\right),
\end{aligned}
$$




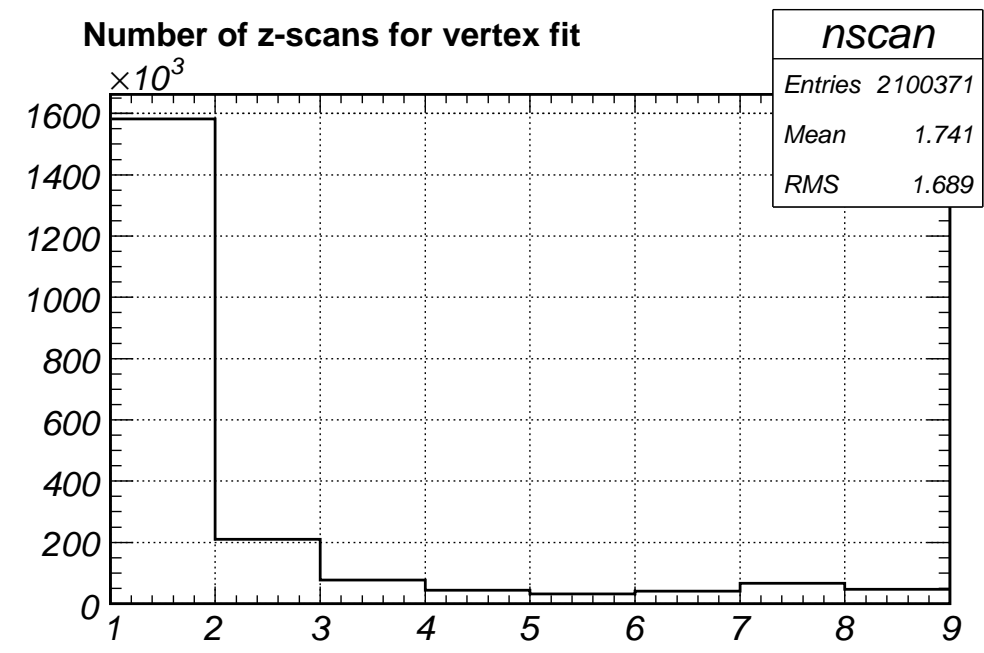

Figure 6.8: Number of $z$ scans required for a vertex constrained fit.

and replacing the relevant part of vector $\vec{v}$ with

$$
v_{i}=\left(\begin{array}{c}
\sum_{j} w_{1 j}\left(u_{i j}-Q_{i} c_{5, i j}\right) c_{3, i j} \\
\sum_{j} w_{1 j}\left(u_{i j}-Q_{i} c_{5, i j}\right) c_{4, i j}
\end{array}\right) .
$$

Otherwise, no changes to the algorithm are required.

\subsubsection{Limitations of Vertex Fitting}

${ }_{\wedge}$ Vertex finder clusters tracks within $\pm 3 \mathrm{~cm}$ of the vertex position. This means that nearby secondary vertices may get erroneously pulled into the primary vertex. The two problems that arise in the pull on vertex position that the incorrect tracks will have and the incorrect conclusion that the extra particles come from the vertex rather from a neutral decay.

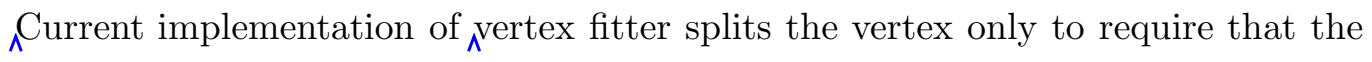
RMS of track times (as measured by drift chamber wire hits) is below 20 ns. This cleans up pileup, but does not do anything to tracks otherwise incorrectly associated to a vertex.

${ }_{\Lambda}^{6} \mathrm{~mm}$ resolution of vertex $z$ (see Figure 6.9 is inferior to $\approx 2 \mathrm{~mm}$ resolution that was obtained by BNL E910 experiment. The primary reason is thought to be imperfect

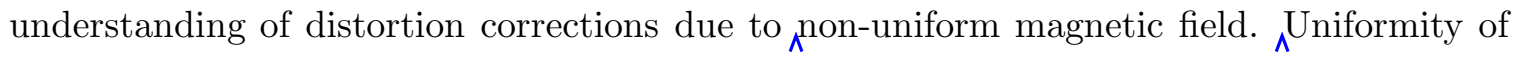


Vertex Z distribution

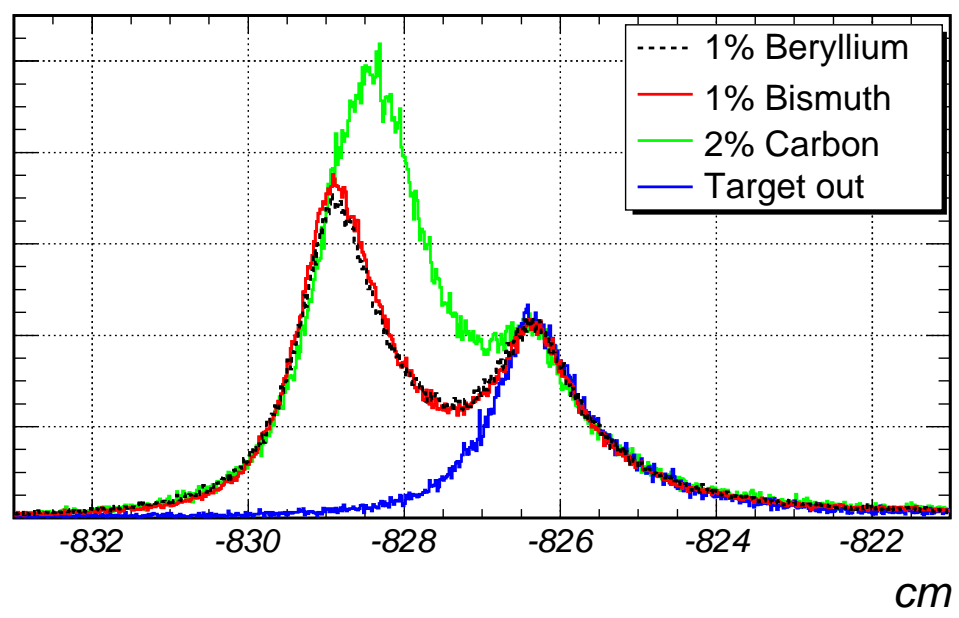

Figure 6.9: Shown are distribution of fitted vertex $z$ from three different targets and target out configuration. The histograms were normalized to have the same height $\theta_{\lambda}$ the interaction counter. Excluding the long tails, resolution is approximately $6 \mathrm{~mm}$.

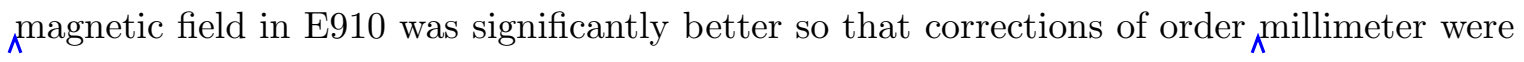

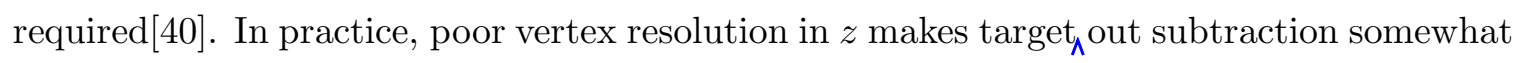
more important for differential cross section measurement, but does not have a large effect on the analysis presented here.

\subsection{Particle Identification}

Particle identification is done using different detector $\overline{\bar{\kappa}}_{\overline{\mathrm{N}}}$ depending on the track momentum. For particles under $1 \mathrm{GeV} / c$, energy loss in the TPC can discriminate between different species. ${ }_{\Lambda}^{T}$ Time of flight wall was designed to work for particles with momentum up to $3 \mathrm{GeV} / c$. Threshold Cherenkov can identify particles with momentum up to around $17 \mathrm{GeV} / c$. Finally, above $20 \mathrm{GeV} / c$, only ${ }_{\Lambda}^{\mathrm{RICH}}$ is able to discriminate different species. Since this work is focused on high momentum end of the spectrum, reconstruction algorithm of the RICH is described here.

\subsubsection{Particle Identification Methods}

Given a track that passes through the ${ }_{\wedge}^{\mathrm{RICH}}$ radiator volume, there are two approaches to determining the particle $\mathrm{ID}_{\boldsymbol{\kappa}}$ 
1. Calculate the ring radius and convert radius and track momentum into particle mass;

2. Calculate the likelihood for electron, muon, pion, kaon, proton hypotheses and select ${ }_{\Lambda}^{\mathrm{ID}}$ of the hypothesis with the largest likelihood.

For most momenta of interest, the two approaches give identical results, as one would expect by looking at Figure 6.17 . The first method has the advantage of being fast, but it does not take the dispersion of radiator gas into account. The second method is slower, but has the capability of improving separation at high momenta where the difference between $\pi / \mathrm{K} / \mathrm{p}$ Cherenkov angles is less than the thickness of the ring. However, this improvement can only be obtained when the detector is very well calibrated. This work is based on calculation of ring radii, and is adequate for momenta where pion and kaon statistics is significant.

\subsubsection{Fitting Ring Radius}

The algorithm to fit ring radii is based on deterministic annealing filter (DAF) coupled to hit sharing algorithm used in $\Lambda_{\Lambda}$ ERA-B RICH [41].

While the algorithm does not take the effect of dispersion of $\mathrm{CO}_{2}$ in detail, the RMS width of the ring as a function of ring radius is taken into account. Figure 6.10 shows that ${ }_{\wedge} \mathrm{RMS}$ of the light distribution can be modeled by a simple function for $r>9 \mathrm{~cm}$ :

$$
\sigma(r)=C \cdot(r)^{-a}
$$

where $C=23.78$ and $a=1.044$ give $\sigma(r)$ and $r$ in $\mathrm{cm}$. For $r \lesssim 9 \mathrm{~cm}$, the number of photoelectrons starts dropping as the photons for higher wavelengths go below Cherenkov threshold. In order to allow the algorithm to converge to radius below $9 \mathrm{~cm}$, the width associated with the ring was modeled as

$$
\sigma(r)= \begin{cases}C \cdot r_{c r i t}^{-a}, & r \leq r_{c r i t} \\ C \cdot r^{-a}, & r>r_{c r i t}\end{cases}
$$

$r_{\text {crit }}$ was chosen to be $8 \mathrm{~cm}$ for reasons explained in Section 6.6.4.

Looking at the index of refraction and PMT efficiency as a function of wavelength of light, one cannot expect that the function of expected photoelectrons to be Gaussian. However for simplicity, the weight of each PMT given the ring radius is taken as

$$
w_{i}(r)=\frac{\left(r-r_{i}\right)^{2}}{\sigma^{2}(r)}
$$




\section{Ring radius vs Momentum}

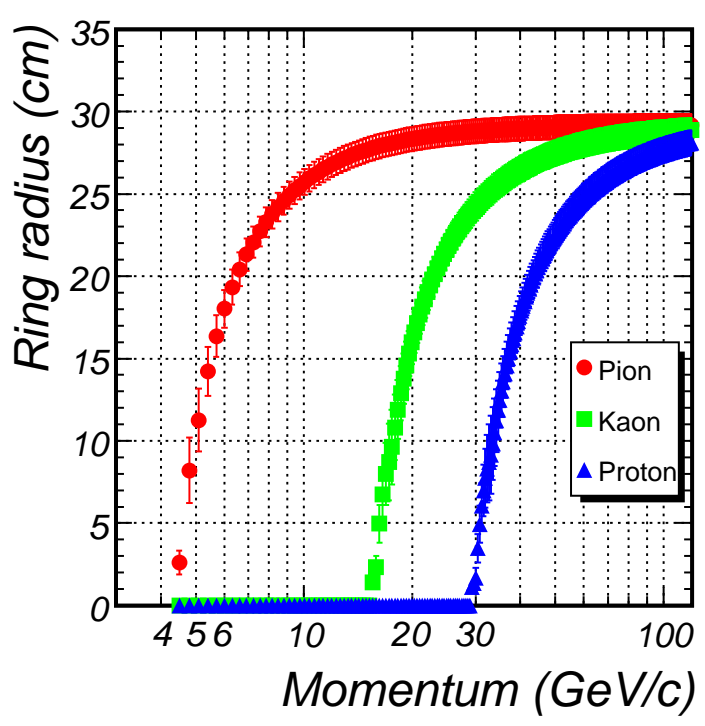

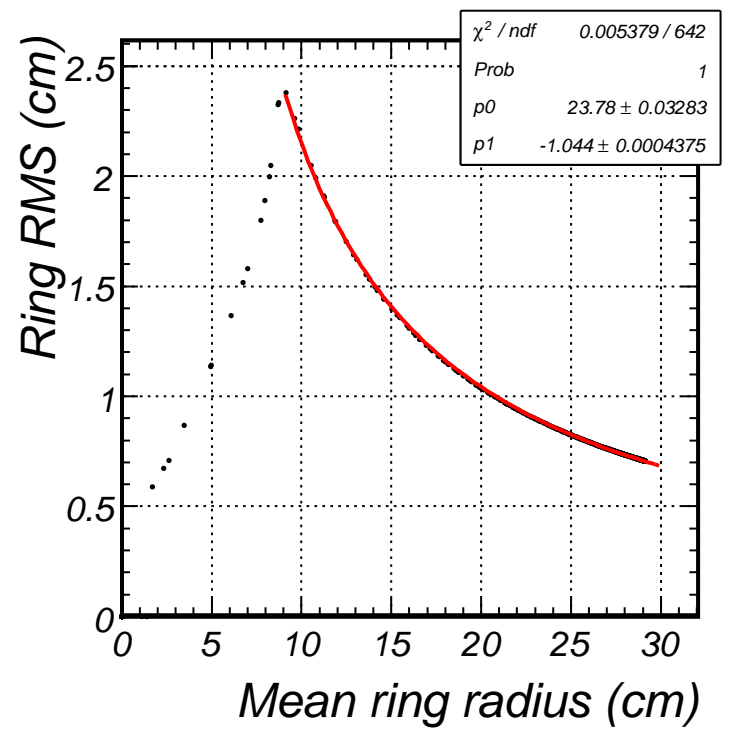

Figure 6.10: Shown are pion, kaon, and proton mean ring radii and RMS of light distribution is used as error $\kappa$
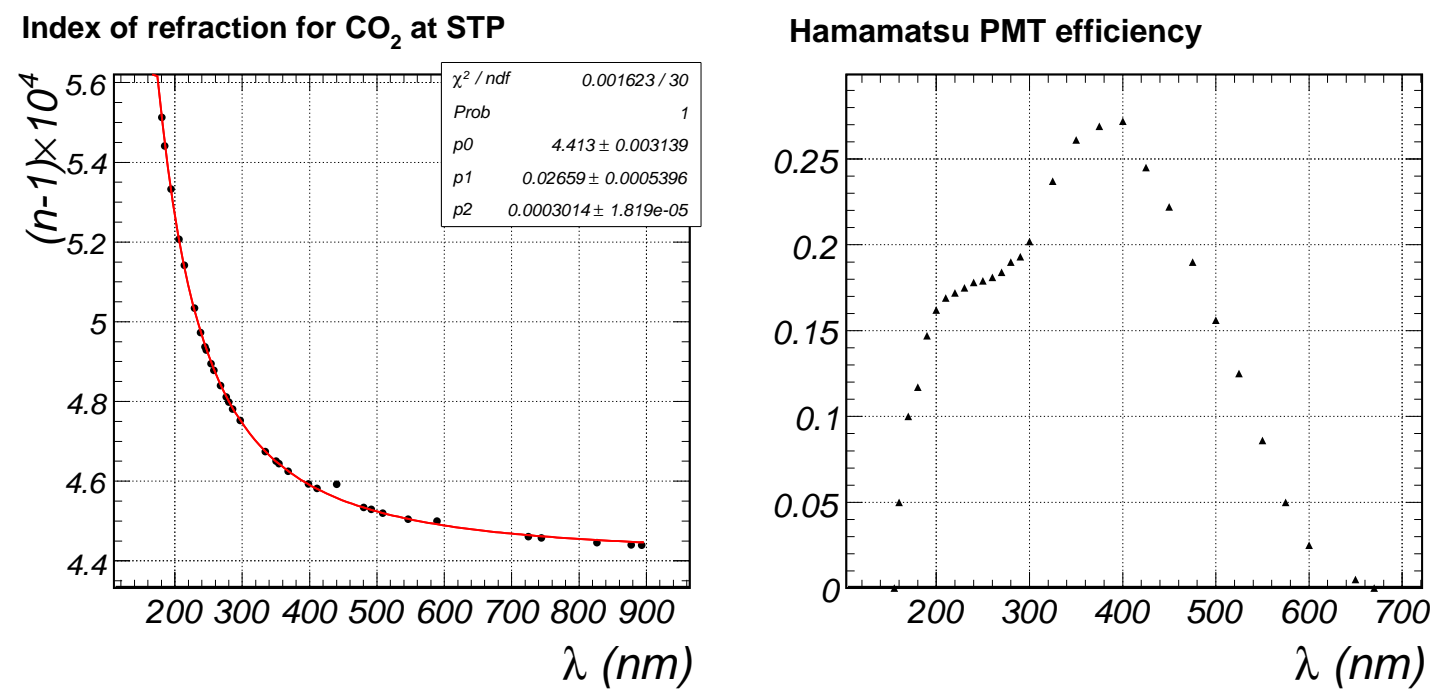

Figure 6.11: Shown are available data for $\mathrm{CO}_{\Lambda}$ index of refraction and efficiency of Hamamatsu PMT's (efficiency shape of FEU's is similar but about a factor of 2 lower). The index of refraction is well fit by $(n-1) \cdot 10^{4}=4.413+2.66 \cdot 10^{-2} \cdot \lambda^{-2}+3.01 \cdot 10^{-4} \cdot \lambda^{-4}$ where $\lambda$ is in $\mu \mathrm{m}$. 
The first step is to select in-time track $\overline{\boldsymbol{\lambda}}_{\bar{\lambda}}$ with non-zero path length through ${ }_{\Lambda} \mathrm{CO}_{2}$, and predicted ring center within maximum (electron) ring radius from the PMT array. For each ring hypothesis, we compute the number of hits within the active region - defined by electron ring radius with a $\sim 5 \mathrm{~cm}$ safety margin. Any track with less than 3 hits in the active region is dropped from the track list.

To determine the seed ring radius, we scan the minimization function $\lambda_{\lambda}$ for radii from $2.5 \mathrm{~cm}$ up to electron radius in steps of $\sigma(r)$ and select the radius with the smallest function value. To limit the effect of outliers, the weight of hits is limited to $\sigma_{\max }^{2}$. Section 6.6.3 explains the reasons why $\sigma_{\max }=3.7$ was chosen.

Equipped with predicted ring radius, we iterate over ${ }_{\Lambda} \mathrm{DAF}$ temperature cycle with the following algorithm:

1. Compute $\sigma_{\max }(r)$, where $r$ is the current guess of the ring radius. It is important not to vary $\sigma_{\max }$ as $r$ changes because that forces minimization to choose smaller radius and produces slightly wider distribution.

2. Compute filter hit weight. The DAF critical radius is taken as $\sigma_{\max }^{2}$.

3. Compute hit occupancy $o_{i ; j}$ in each ring, which is defined as the number of PMT's that fall in to the same radius bin of $j$-th track. $0.4 \sigma$ bins were used.

4. Fit each ring radius allowing ring center position to vary if at least 6 hits are associated with the ring. Each hit weight is modified as $w_{i ; j}=w_{i}(r) \cdot w_{D A F} \cdot \frac{o_{i ; j}}{\sum_{j} o_{i ; j}}$,

thus the total weight of each hit is 1 , but varies in different tracks so that hit sharing is taken into account.

The DAF temperature cycle of $(5,1,0.2,0.04) \mathrm{cm}^{2}$ was found to work well even in busy events. At the end of the cycle, hit usage was computed with $\Lambda^{0} \mathrm{~cm}^{2}$ DAF critical radius where hits with weight larger than 0 were considered to be used by the algorithm. Rings that had less than 2 non-shared hits were interpreted to come from noise and were assigned $r=0$.

\subsubsection{Choosing $\sigma_{\max }$}

${ }_{\Lambda} \mathrm{DAF}$ critical radius parameter $\sigma_{\max }$ dictates the signal region for a given ring radius. There are two conflicting considerations that must be taken into account to select 

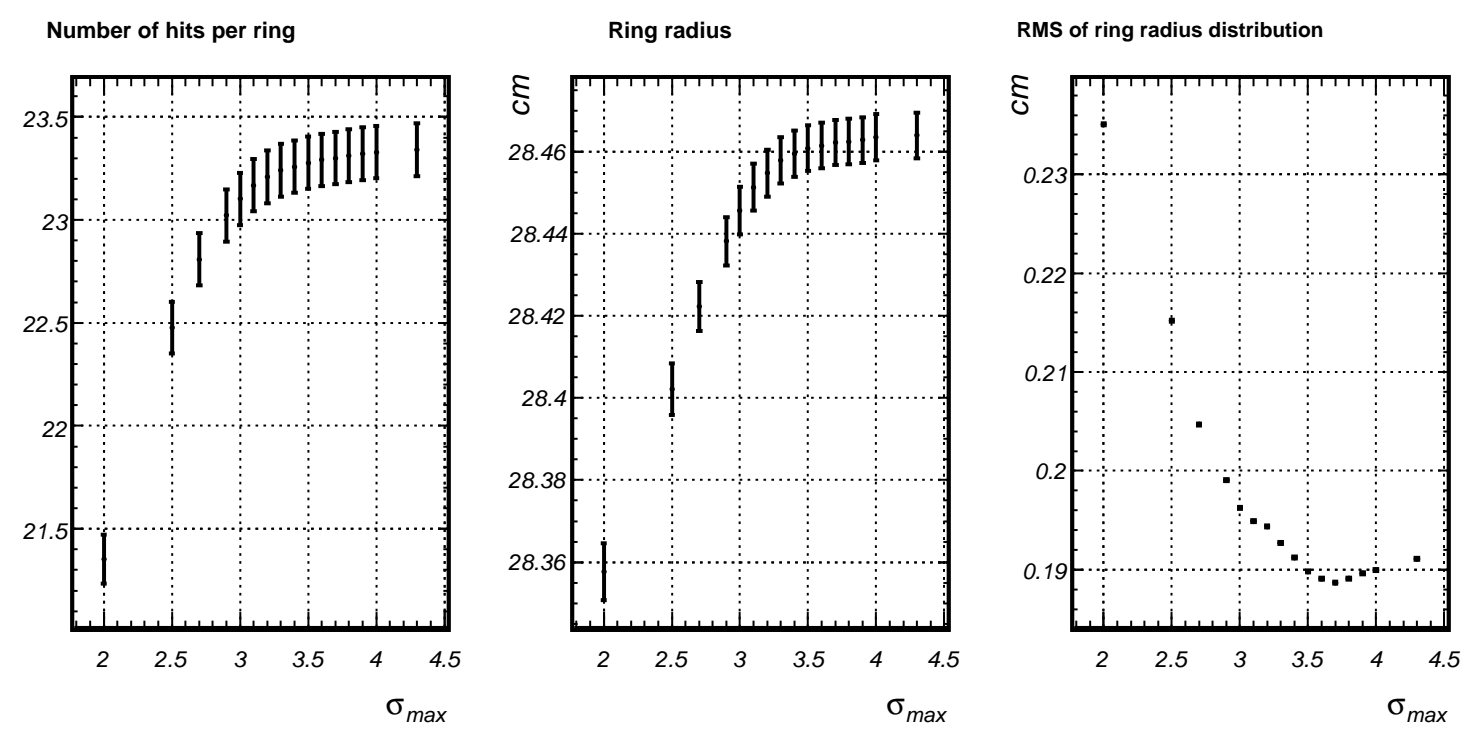

Figure 6.12: Shown are effects on the proton ring fits by different choices of $\sigma_{\max }$ (see text for explanation).

its value. On the one hand, the region should be sufficiently large to include all hits that come from the ring. On the other hand, very large $\sigma_{\max }$ will be more susceptible to noise.

Figure 6.12 shows how proton ring fits are affected by the choice of ring signal region. We see that smaller values of $\sigma_{\max }$ prevents the algorithm from using all of the available information for the ring fit, resulting in fewer hits on a ring, smaller radius, and larger width of ring distribution. As $\sigma_{\max }$ is pushed past 3.7, the potential for picking up noise is higher, resulting in somewhat larger width of the proton ring distribution.

\subsubsection{Incorrectly Fit Rings}

A feature of the algorithm is that it will preferentially go for hits inside the signal region, whether those hits were generated by noise or particle Cherenkov light. There are two predictable ways in which the algorithm will incorrectly fit a ring

1. Shift ring center far away from predicted position;

2. Fit maximally allowed ring radius, well above electron ring radius.

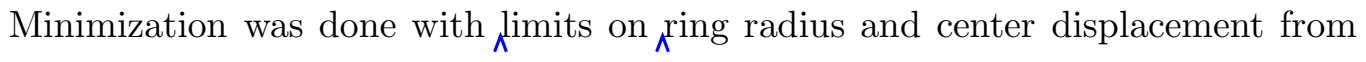

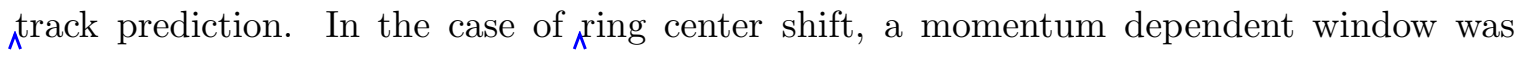




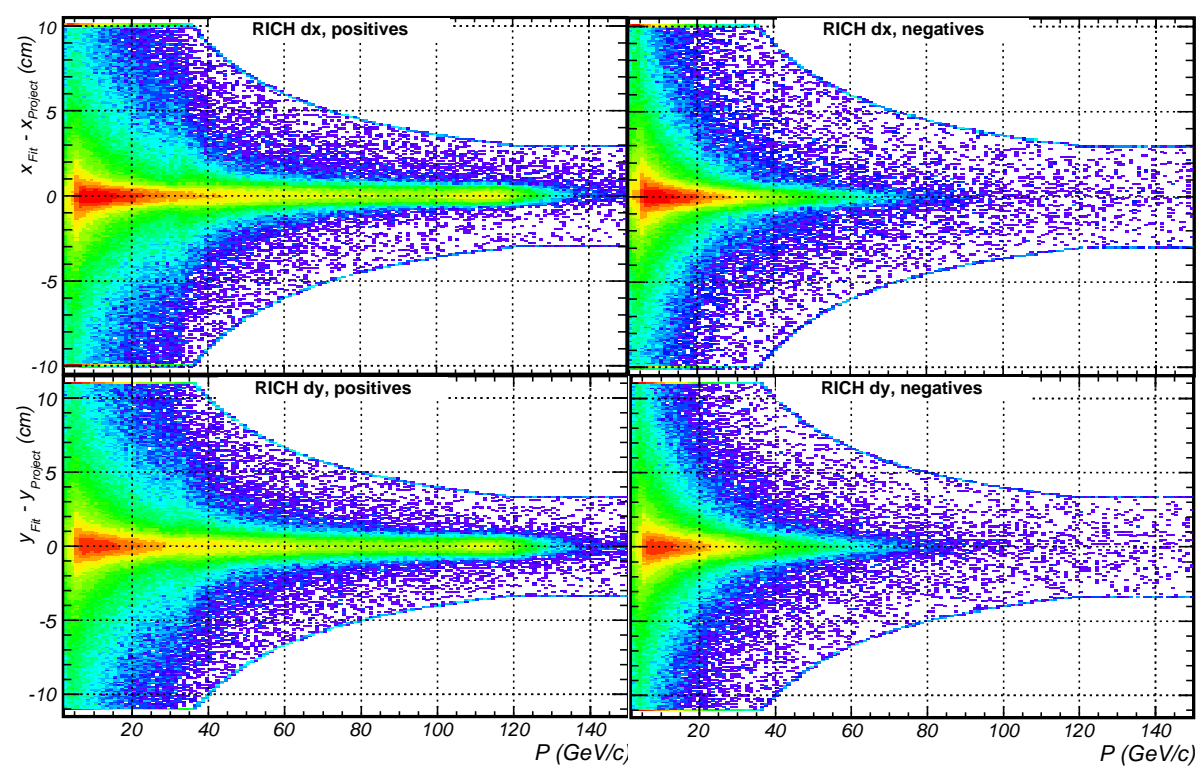

Figure 6.13: Shown are distributions of ring center displacement in $x$ and $y$ by the fit from the prediction made by incoming track.

chosen with

$$
D_{x, y}= \begin{cases}C_{x, y} / p_{t o t}, & p_{t o t}<120 \\ C_{x, y} / 120, & p_{t o t} \geq 120\end{cases}
$$

where $C_{x}=360 \mathrm{~cm} \mathrm{GeV} / c, C_{y}=400 \mathrm{~cm} \mathrm{GeV} / c$, and $p_{t o t}$ is momentum of the track. The choice is motivated by effects of multiple scattering, since the track position and angle predictions are more reliable for higher momentum tracks. Finally, $D_{x}$ was limited to $10 \mathrm{~cm}$ and $D_{y}$ to $11 \mathrm{~cm}$ so that the ring would be less likely to "walk" into another ring potentially causing two incorrectly fit rings.

\subsubsection{Corrections to Ring Radius}

When the RICH was repaired after the accident, every fourth PMT column was left empty. This creates aliasing in ring fits due to regularly missing information. Effect on ring fits can be observed by scanning over pion ring radius selecting events in a narrow band of momentum so that predicted ring radius does not change by more than $\pm 0.1 \mathrm{~cm}$. Figure 6.14 shows that observed ring radius depends on the mean value of ring radius and the $x$-coordinate of the ring center. The period of the sinusoidal variations is $5.5 \mathrm{~cm}-$

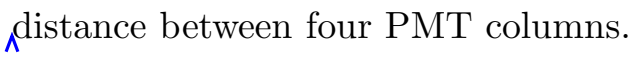




\section{$\mathrm{R}$ vs $\mathrm{x}$ for pion rings}

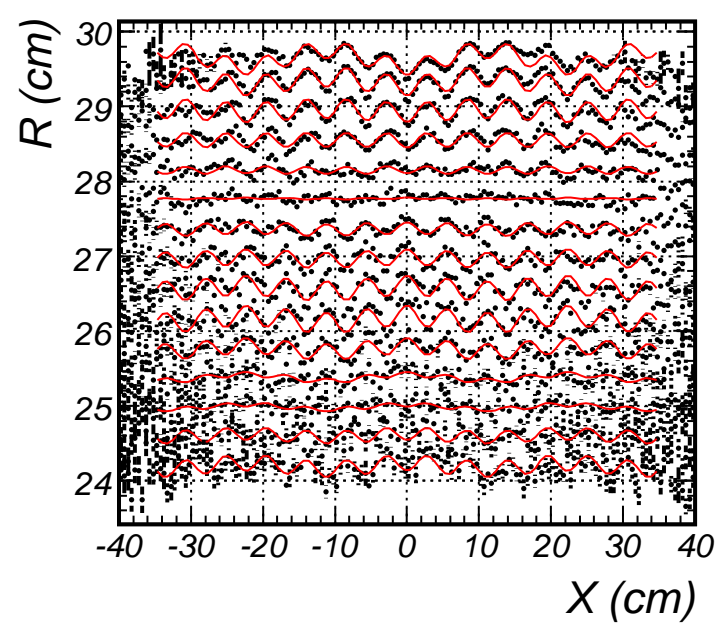

$R$ vs $y$ for pion rings

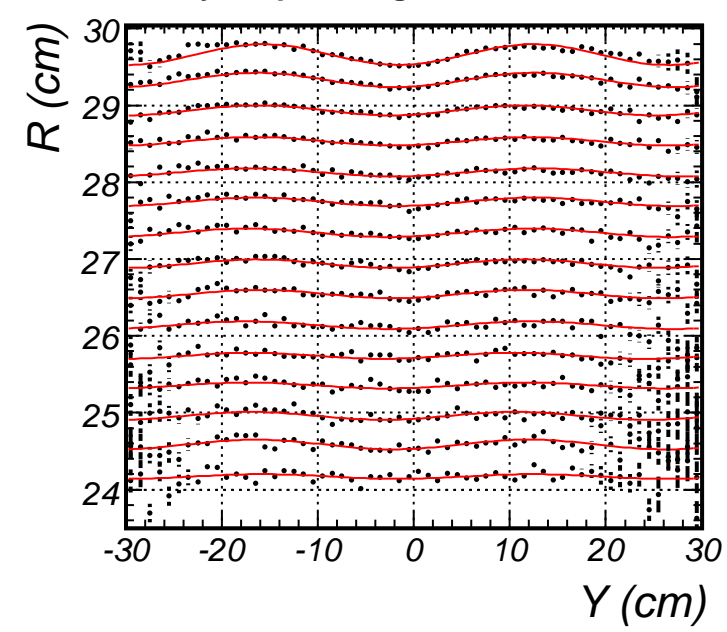

Figure 6.14: Ripples in measured ${ }_{\Lambda} \mathrm{RICH}$ ring radius due to aliasing. The binned histograms shown result from $\Lambda_{\Lambda}$ scan of pion rings in $4 \mathrm{~mm}$ steps of central ring radius.

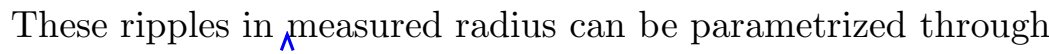

$$
\begin{aligned}
& \delta R_{x}(x, R)=A_{x 0}(R) \cos \left(\omega_{x} x\right)+A_{x 4}(R) \cos \left(\frac{\omega x}{4}\right), \\
& \delta R_{y}(y, R)=A_{y}(R) \cos \left(\omega_{y} y+\phi\right) .
\end{aligned}
$$

Lower frequency corrections in $x$ as well as correction in $y$ result from systematic difference in ring radius as a portion of the ring is no longer detected by photomultipliers as the ring center shifts away from the center of the PMT array. As Figure 6.15 shows, when we do that we find that the amplitude $A_{x 0}(R)$ is well described by $A_{0} \sin (\omega R)$. This corroborates the statement that ripples result from geometric aliasing.

Ripple corrections are most important for high radius rings when at high momenta the difference in ring radii between protons, kaons, and pions becomes small. Figure 6.16 shows that for high momentum pions the effect is a $\sim 10 \%$ improvement in the RMS of ring distribution.

\subsubsection{Particle Identification from Ring Radius}

For a Cherenkov photon in a gas with index of refraction $n$, the angle at which photon is emitted by a particle with speed $\beta$ is given by

$$
\frac{1}{2} \theta^{2}=1-\frac{1}{n \beta}
$$



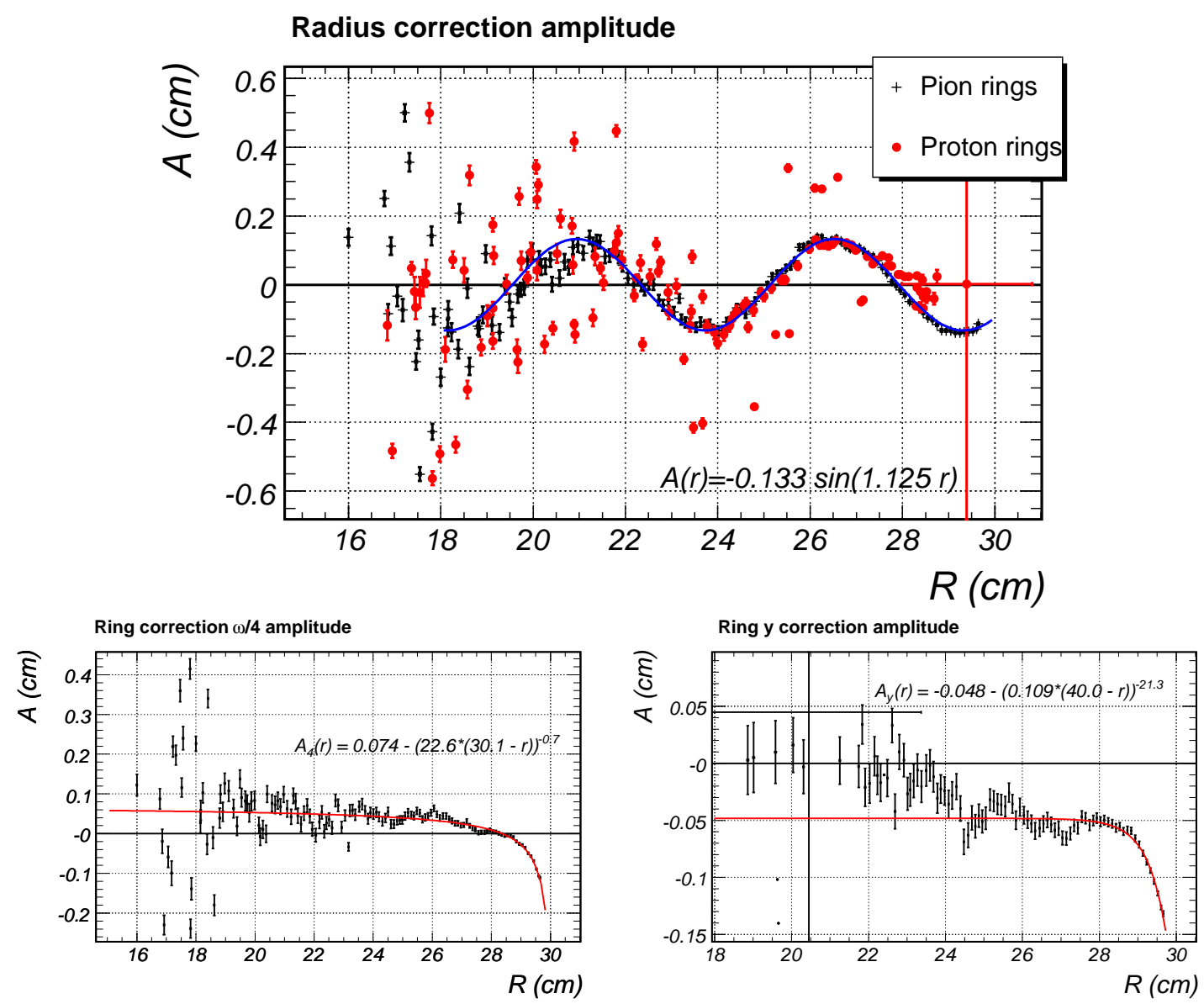

Figure 6.15: Results of fits to amplitude of ring radius ripples as a function of central ring radius. As a sanity check, the scan was repeated for proton rings to verify that the effect is not specific to pion rings. The fact that amplitude of the dominant oscillation in $x$ is consistent with $\sin \left(\omega_{x} R\right)$ confirms that the effect is purely geometric. 


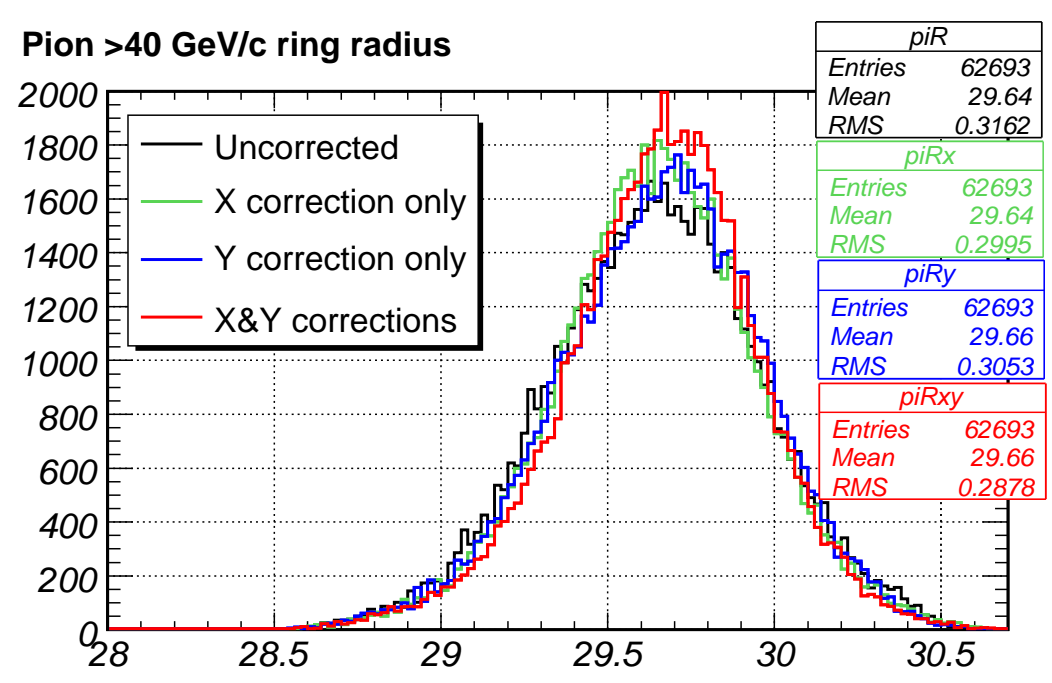

Figure 6.16: A 10\% improvement in radius width of high momentum pions is obtained by removing ripple corrections through parametrized equations.

We can define $\theta_{0}$ as the maximum opening angle, when $\beta=1$. Then

$$
\frac{1}{2} \theta_{0}^{2}=1-\frac{1}{n}
$$

From ${ }_{\wedge}$ equation of relativistic momentum $p=m \gamma \beta$, we get

$$
m^{2}=p^{2}\left[\frac{1}{\beta^{2}}-1\right]=p^{2}\left[\left(\frac{1-\frac{1}{2} \theta^{2}}{1-\frac{1}{2} \theta_{0}^{2}}\right)^{2}-1\right]=p^{2}\left[\left(\frac{F^{2}-\frac{1}{2} R^{2}}{F^{2}-\frac{1}{2} R_{0}^{2}}\right)^{2}-1\right],
$$

where $F=990 \mathrm{~cm}$ is the focal length of RICH mirrors, and $R_{0}$ is the ring radius of $\beta=1$ particle. Given the fact that $n \approx 1+4.5 \cdot 10^{-4}$ in $\mathrm{CO}_{2}$ at STP, $\theta_{0}^{2} \approx 9 \cdot 10^{-4}$. Equation 6.25 can then be approximated to better than one part in a thousand by

$$
m^{2}=p^{2} \frac{R_{0}^{2}-R^{2}}{F^{2}}
$$

which is handy to get a feel for behavior of the ring radius on momentum. Using equation 6.25 we can then transform ${ }_{\wedge} R$ vs $p$ plot into a more useful $m^{2}$ vs $p$ plot, which can be used for an analysis, 

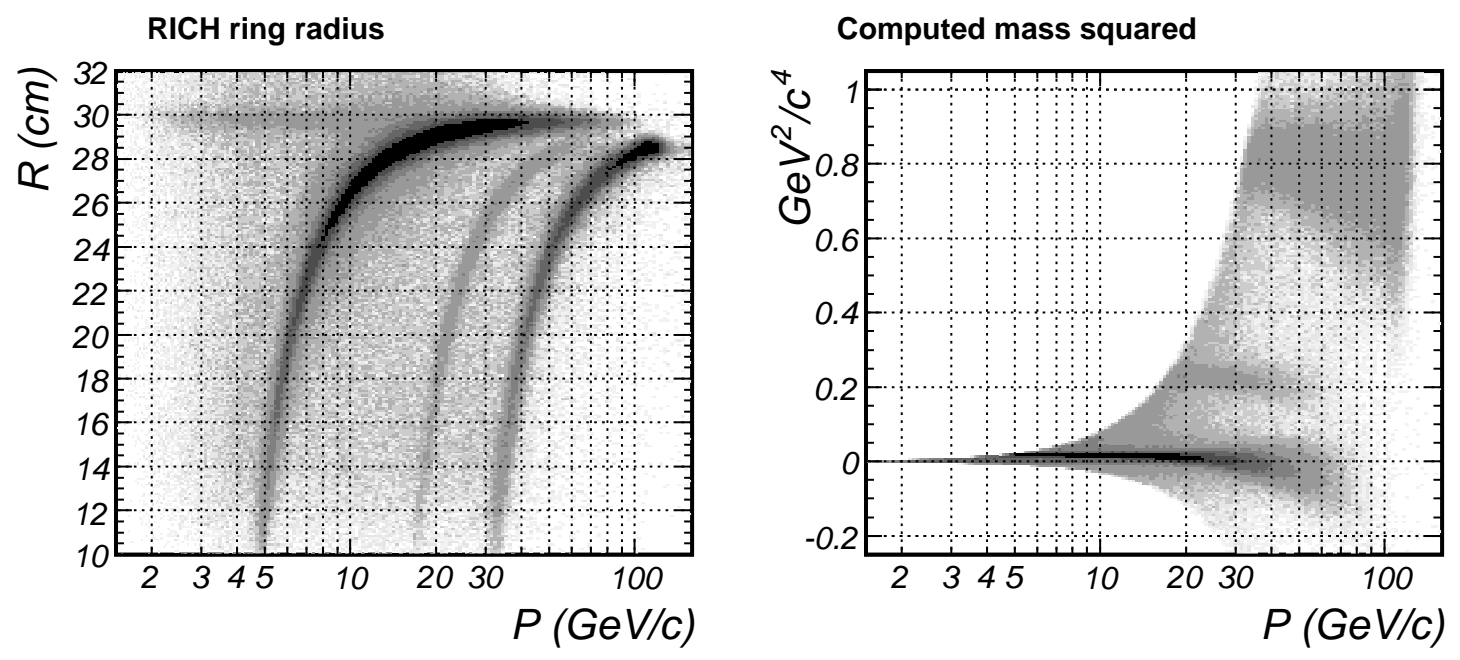

Figure 6.17: Shown is distribution of RICH ring radii in $120 \mathrm{GeV} / c$ interactions (left) and the same plot where mass squared is computed using Equation 6.26. 


\section{Chapter 7}

\section{Detector Calibration and Alignment}

Good detector calibration is essential for reconstruction algorithms to work well. We are calibrating different parts of the spectrometer in multiple passes, each subsequent pass depends on constants generated in the previous passes.

In the first pass, we perform calibration of systems that do not require tracking: ADC pedestals, event trigger time, time offsets of drift chamber wires, hot channels in the RICH and wire chambers.

With calibrated drift chamber time offsets, reconstruction is able to improve signal to noise ratio, we are able to align wire chambers, and later align other subsystems (TPC, RICH, calorimeters) to the chambers.

\subsection{Event Time}

Good event time is critical to performance of the TOF wall and $K p$-separation in $5 \mathrm{GeV} / c$ beam. In order to have 3 -sigma $K_{\wedge} p$-separation out to $3 \mathrm{GeV} / c$, one needs 200 ps resolution on $\wedge_{\Lambda}$ time difference measurement (see Figure 7.1).

One of the complications in $\Lambda_{\Lambda}$ calibration of ${ }_{\Lambda}$ T0 counters is temperature dependence of the time offset, requiring time-dependent offset for each channel. Figure 7.2 shows an example of an overnight run where the delay cables drifted by about 1 ns with respect to

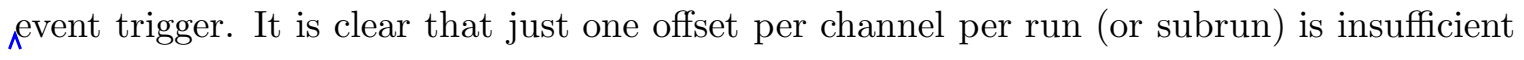
since desired resolution is 200 ps. 


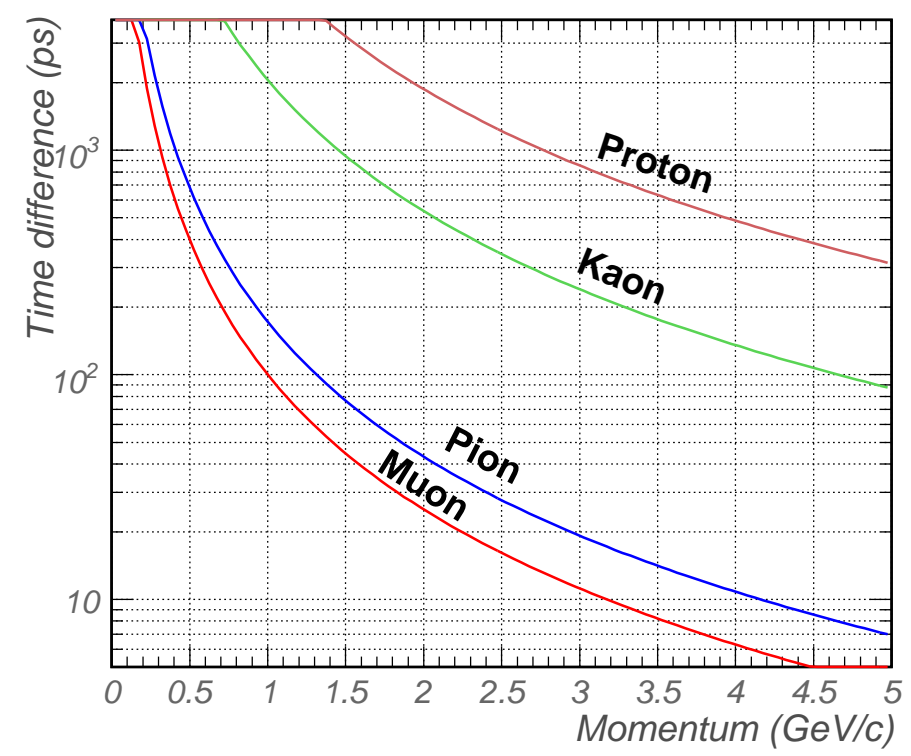

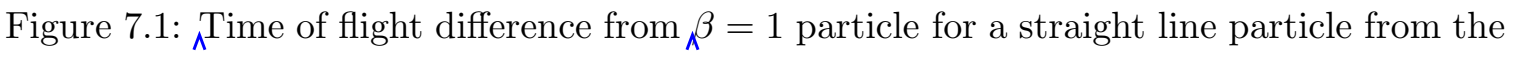
target to $\boldsymbol{\Lambda}^{\text {time }}$ of $_{\Lambda}$ flight wall.

We represent the offset of each channel as a third degree polynomial for each subrun (typically about 5000 events). Coefficients of polynomials are calculated using the following algorithm.

1. Calculate pedestals.

2. Determine ${ }_{\Lambda} \mathrm{TDC}$ offset for every PMT and trigger bit. Different trigger bits were timed in to a nanosecond or so, which is good for trigger system, but these offsets are comparable to the effect we want to correct.

3. ${ }_{\Lambda}$ DC offset of the trigger bit with the largest number of entries is chosen as a reference and relative trigger bits are computed for each counter (group of 4 PMT's).

4. Partition data into bursts. Each burst contains at least 80 triggers (if possible) and lasts no more than 10 minutes. Data is first split into spills, and then spills with less than 80 events are merged with adjacent spills unless the burst would become too long. Average time (seconds since Jan 1, 1970) is computed for each burst. 

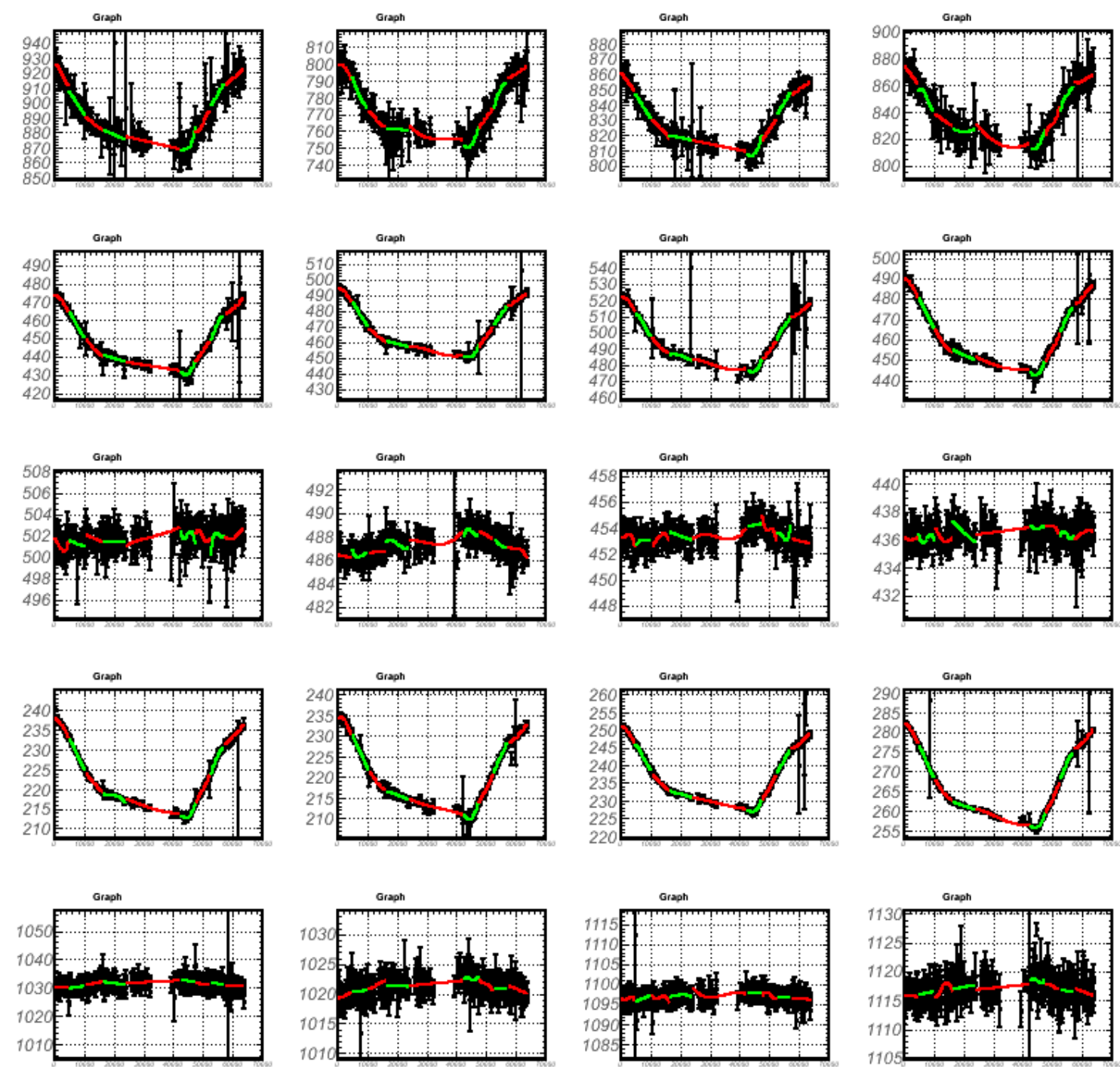

Figure 7.2: Variations in ${ }_{\Lambda}$ T0 counters timing offset for an overnight run and superimposed correction curves for every subrun. Each of the five columns shows distribution for the four PMT's that belong to the counter and set of cables. From top to bottom, the rows correspond to T00-ribbon, T01-ribbon, T01-RG8, TBD-ribbon, T00-RG8. TDC counts are plotted on the vertical axis, with $60 \mathrm{~ns}$ per bin for TBD-ribbon, and $30 \mathrm{~ns}$ per bin for all other counters. 
5. For each burst, ${ }_{\Lambda}$ TDC distribution of every channel is fitted to a Gaussian, and the mean and the error on the mean are saved.

6. The distribution of TDC offsets of every channel is fitted to a third degree polynomial.

7. Steps 2 and 3 are redone using the fits from step 6 . Then the final fits (steps 5 and 6) are obtained and results are stored into the database.

The second correction applied to the TDC signals is time slewing from the amount of charge that creates the signal. Figure 7.3 shows that there is a systematic dependence of ${ }_{\Lambda}^{\mathrm{PMT}}$ hit time on the amount of charge that the PMT collected. Time slewing correction is computed with the following algorithm:

1. Pedestal-subtracted ADC spectrum is fitted to Landau function for each channel.

2. Entries between 0.5 and 2.5 of Landau $\mathrm{MPV}_{\wedge}$ are used to make a graph for every channel. Entries get sorted by $1 / \sqrt{A D C}$, and ${ }_{\Lambda} \mathrm{TDC}$ distribution of at least 40 entries is fitted to a Gaussian, and is saved along with the average 1/sqrtADC.

- The reason for limiting the range of ADC signals is to avoid over-correcting very small signals and limit the impact of pileup on high-ADC events.

3. Distribution for each channel is fitted to a parabola.

4. To ensure that the TDC distribution is centered at 0 once all corrections are applied, an overall offset for each channel is calculated with the temperature and ADC correction taken into account before results are saved into the database.

Results of these corrections give 120 ps resolution for ${ }_{\Lambda}$ T01-TBD time difference (see Figure 7.4, which is sufficient for both ${ }_{\Lambda}$ TOF wall and beam time of flight at low momenta.

\subsection{Drift Chamber Time Offsets}

Between the drift chamber electronics and difference in cable length, time offset had to be calculated for each wire. In addition, there are systematic shifts from run to run, thus effectively we need a constant for each wire and run. Of course, no single run had enough statistics to determine the offset for each wire, so we used the following approach to compute time offsets $\left(t_{0}\right.$ 's). 

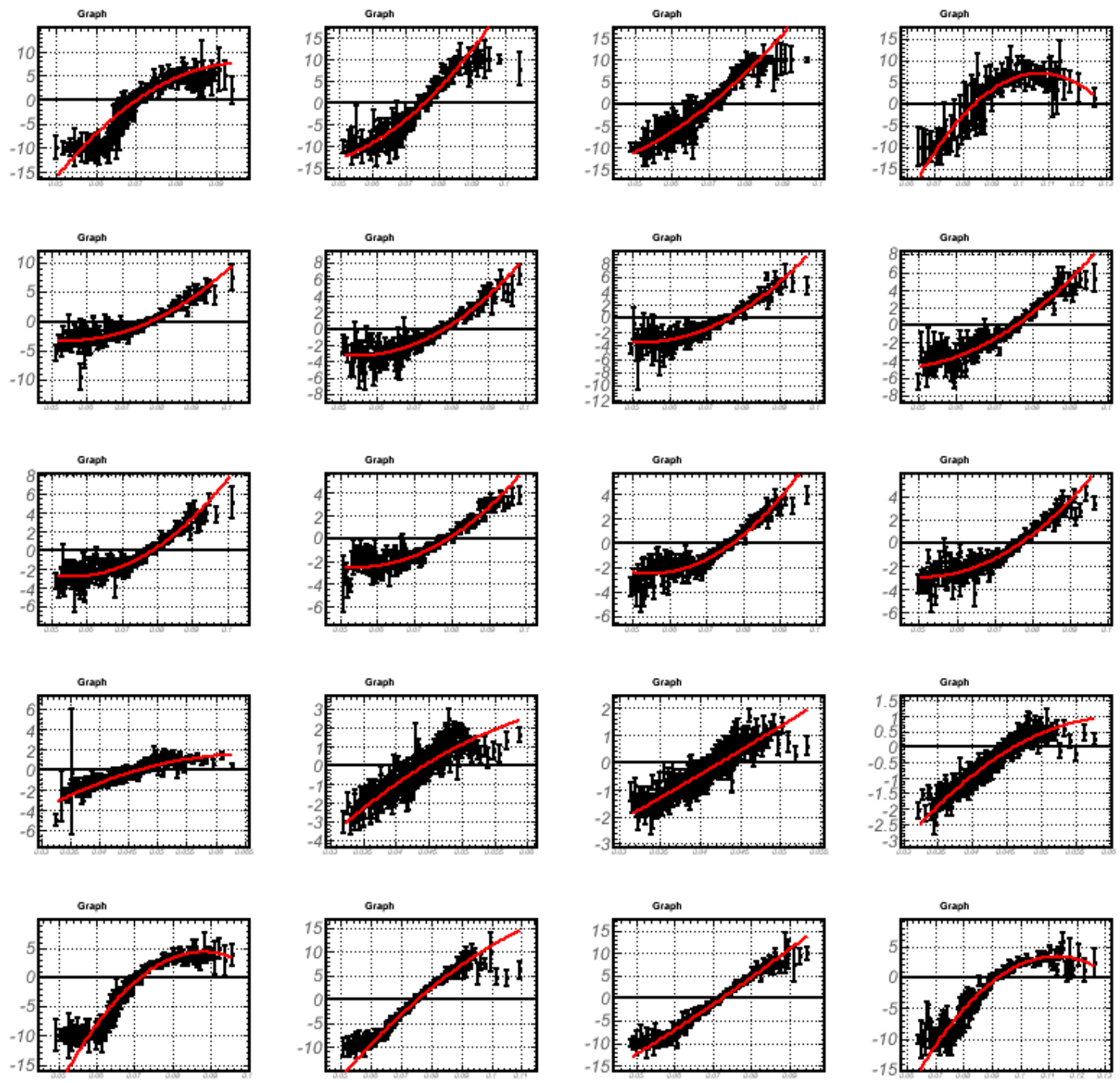

Figure 7.3: Plotted are TDC after temperature correction versus $1 / \sqrt{A D C}$ and superimposed quadratic fits. The five columns are identical to those in Figure 7.2. 


\section{T01 RG8 - TBD Time Difference}

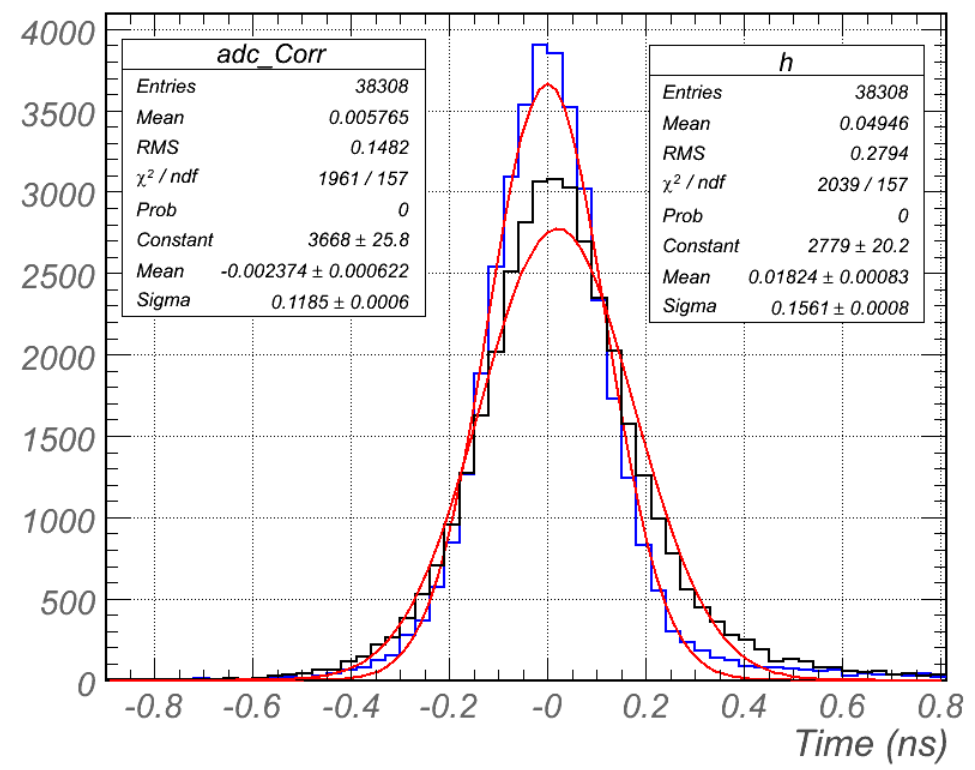

Figure 7.4: Reconstructed time difference between ${ }_{\Lambda}$ T01 and TBD counters with (blue curve) and without (black curve) ADC correction. Time slewing correction improves the resolution by more than $25 \%$. 
1. In each run we attempt to determine $t_{0}$ for wires which have enough statistics;

2. For each run where at least 3 wires are fit, we compute the average $t_{0, \text { run }}$ for the run;

3. For every wire in the runs which have the run offset computed, we compute overall offset for the wire $\left(t_{0, \text { wire }}\right.$ by shifting entries from different runs by $t_{0, \text { run }}$.

4. For every run, we use wires with $\Lambda_{\text {valid }} t_{0, \text { wire }}$ to recalculate $t_{0, \text { run }}$;

5. As steps 3 and 4 are repeated three times, each subsequent iteration picks up more runs and wires;

6. For every wire in every run, time offset is set to $t_{0}$ if it was computed in step 1 , otherwise it is set to $t_{0, \text { run }}+t_{0, \text { wire }}$, thus taking into account differences from run to run and from wire to wire.

\subsubsection{Event Selection}

Event selection is quite important in order to determine $t_{0}$ 's well. We attempt to limit pileup by imposing the following requirements:

- No more than two $\mathrm{T}_{3 / 4}$ or $\mathrm{TBD}_{3 / 4}$ are allowed in the event;

- If there are two $3 / 4$ signals in either counter, time separation between the two has to be at least $100 \mathrm{~ns}$;

- If a beam chamber has more that 24 wires hit, it is ignored;

- If a drift chamber has more 150 wire hit, it is ignored.

Although a significant fraction of $\lambda_{\Lambda}$ ata is thus ignored, the signal to noise ratio is much higher than if all the available data are used. Given the fact that wires on the edge of the chamber have little signal, it is extremely important to reject noise.

\subsubsection{Fitting}

Wire time distributions can be modeled quite well by a Gaussian with exponential tail, i.e.

$$
f(t)=\left\{\begin{array}{cc}
A \cdot \exp \left(-\frac{1}{2}\left(\frac{t-t_{\text {peak }}}{\sigma_{t}}\right)^{2}\right), & t<t_{\text {peak }}+\sigma_{\text {cut }} \sigma_{t} \\
A \cdot \exp \left(\frac{1}{2} \sigma_{\text {cut }}^{2}-\frac{t-t_{\text {peak }}}{\sigma_{t}} \sigma_{\text {cut }}\right), & t \geq t_{\text {peak }}+\sigma_{\text {cut }} \sigma_{t}
\end{array}\right.
$$


Here $t_{\text {peak }}$ is the usual Gaussian peak, and $\sigma_{c u t}$ is a unitless parameter which determines how many sigma to the right of the peak the function becomes exponential. In this representation, the function and its derivative are continuous. After making a guess at parameters

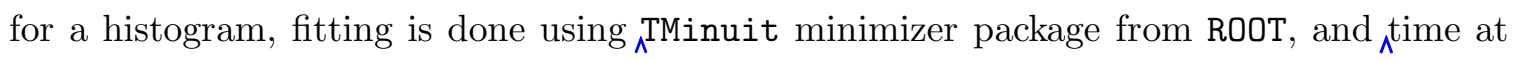
half-maximum was taken as $t_{0}$.

\subsection{Chamber Alignment}

\subsubsection{Aligning Chamber Planes}

Initial chamber plane positions from the survey of the experimental hall agreed with data to within a few a few millimeters. Misalignment of chamber planes was sufficiently small, so that track finding efficiency was not affected too much, but as Figure 7.5 shows, improvement to alignment were necessary. To put matters in perspective, ${ }_{\Lambda} 120 \mathrm{GeV} / c$

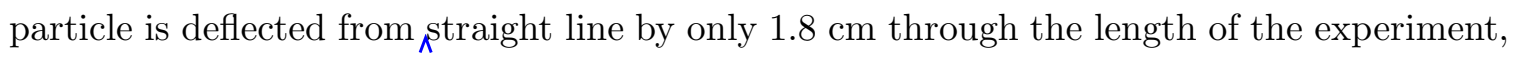
so a half wire spacing $(1.5 \mathrm{~mm})$ misalignment of leads to a $10 \%$ systematic effect on the measured momentum.

${ }_{\Lambda}$ Chamber alignment is split into two parts:

- Align ${ }_{\text {beam }}$ chambers,

- Align chambers 1-6 to beam chambers.

Doing alignment for beam chambers separately boosts statistics for the 3 chambers, since a

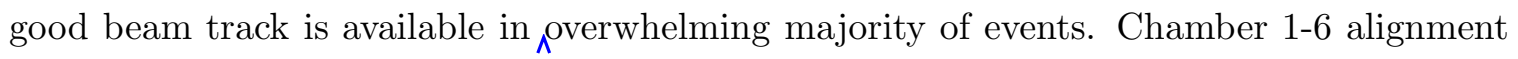
is done with $6_{\lambda}$ chamber tracks and 9-chamber beam tracks. The first set of tracks makes it possible to determine chamber rotations relative to one another (BCs do not have a long enough lever arm), and the second set makes sure that alignment of all 9 chambers is consistent.

Track selection is crucial to successful alignment calculation. For the final pass, we select only those tracks that pass through all 6 chambers and where at most two chamber planes are missing. Requiring almost all planes to be present provides immunity to incorrectly reconstructed tracks and reduces the bias due to insufficient amount of data. Unfortunately, chamber efficiency is not high enough to allow us to use only those tracks where all chamber planes were present. If a wire plane containes two hit wires, it is excluded 
Chamber residuals

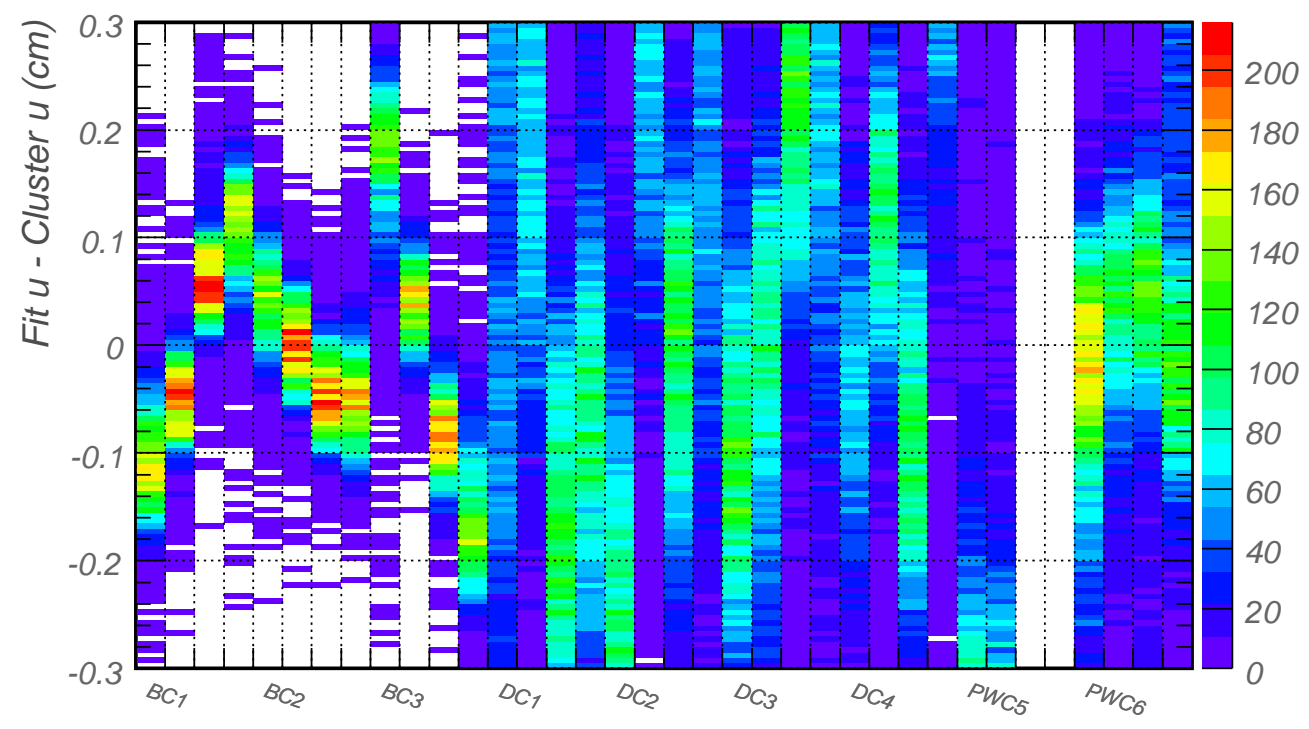

Figure 7.5: Chamber residuals before the chamber alignment was done. On the horizontal axis are chamber planes, on the vertical ${ }_{\bar{\lambda}}$ residuals from 9 -chamber track fits.

from the fit so as to limit the impact of noise and pileup. Lastly, tracks below $4 \mathrm{GeV} / \mathrm{c}$ are rejected, because of significant multiple-scattering, and the fact that continuous energy loss in the spectrometer is not taken into account. This requirement is not a heavy toll on available statistics because of poor acceptance for low momentum particles in chamber 6 .

The alignment algorithm is quite simple. For each wire in the track we calculate

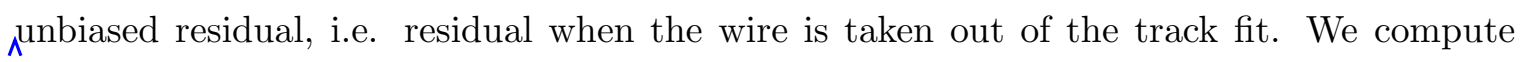
the mean of residuals for each wire plane using all available tracks, and shift the wire plane by 0.3 of the residual. Next the track list is preened by removing those tracks where any one of residuals exceeded 1.5 wire spacing plus the absolute value of computed mean. The procedure is repeated until the largest mean of residuals is below $1 \%$ of wire spacing.

A typical run with at least 5000 tracks provided enough statistics to do reliable alignment $_{\overline{\bar{\lambda}}}$ to monitor stability and correlation with beam momentum. 


\section{Chamber residuals, post-alignment}

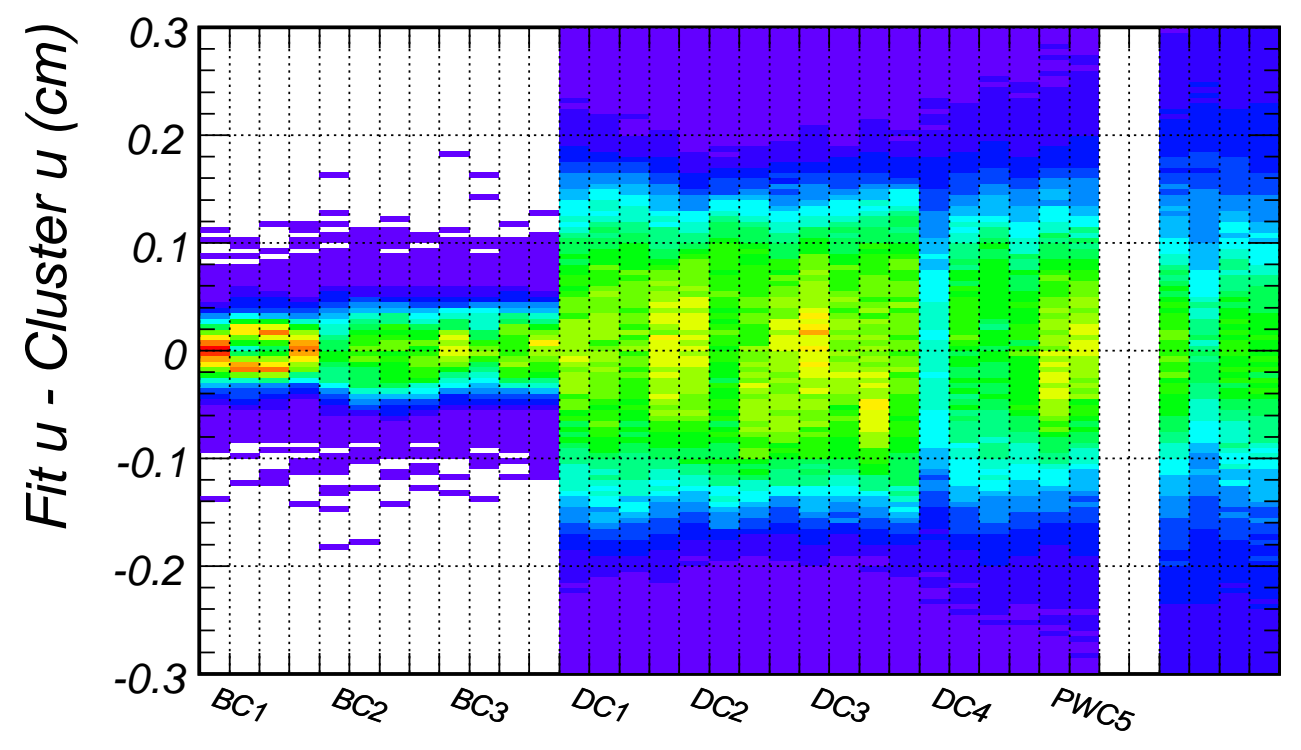

Figure 7.6: Residuals once chamber alignment was completed.

\subsubsection{Z-alignment and Rotations}

A lot of effort went into verifying that chamber $z$-positions and rotations about $z^{-}$

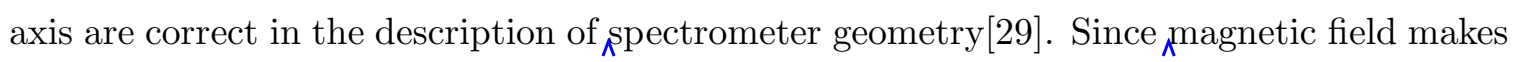
track fits more uncertain, we relied upon field $\mathrm{A}_{\mathrm{ff}}$ data. Unfortunately, the amount of available field $\alpha_{\Lambda}$ off data is very small, so precision of field-off alignment is limited. Nevertheless, this work helped find major errors in positioning of ${ }_{\Lambda}$ chambers in the experiment.

The algorithm for alignment of chambers is similar to wire plane offset: tracks are stored in memory for the entire run, and at the end minimization of the sum of $\chi^{2}$ is done using TMinuit by shifting $z$ of the chambers and rotating them about $z$-axis.

Figure 7.7 shows that even when all known errors were solved in the geometry

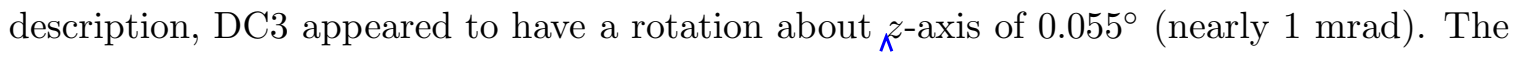
effect of this rotation on the edge of the chamber is about $\frac{1}{4}$ of wire spacing, which is noticeable, so this rotation was corrected.

Figure 7.8 shows results of $k^{z}$-alignment algorithm re-run with DC3 rotated. Here we see that the alignment algorithm wants to move DC123 in $z$ by $5 \mathrm{~mm}, 3 \mathrm{~mm}$, and $2 \mathrm{~mm}$ 

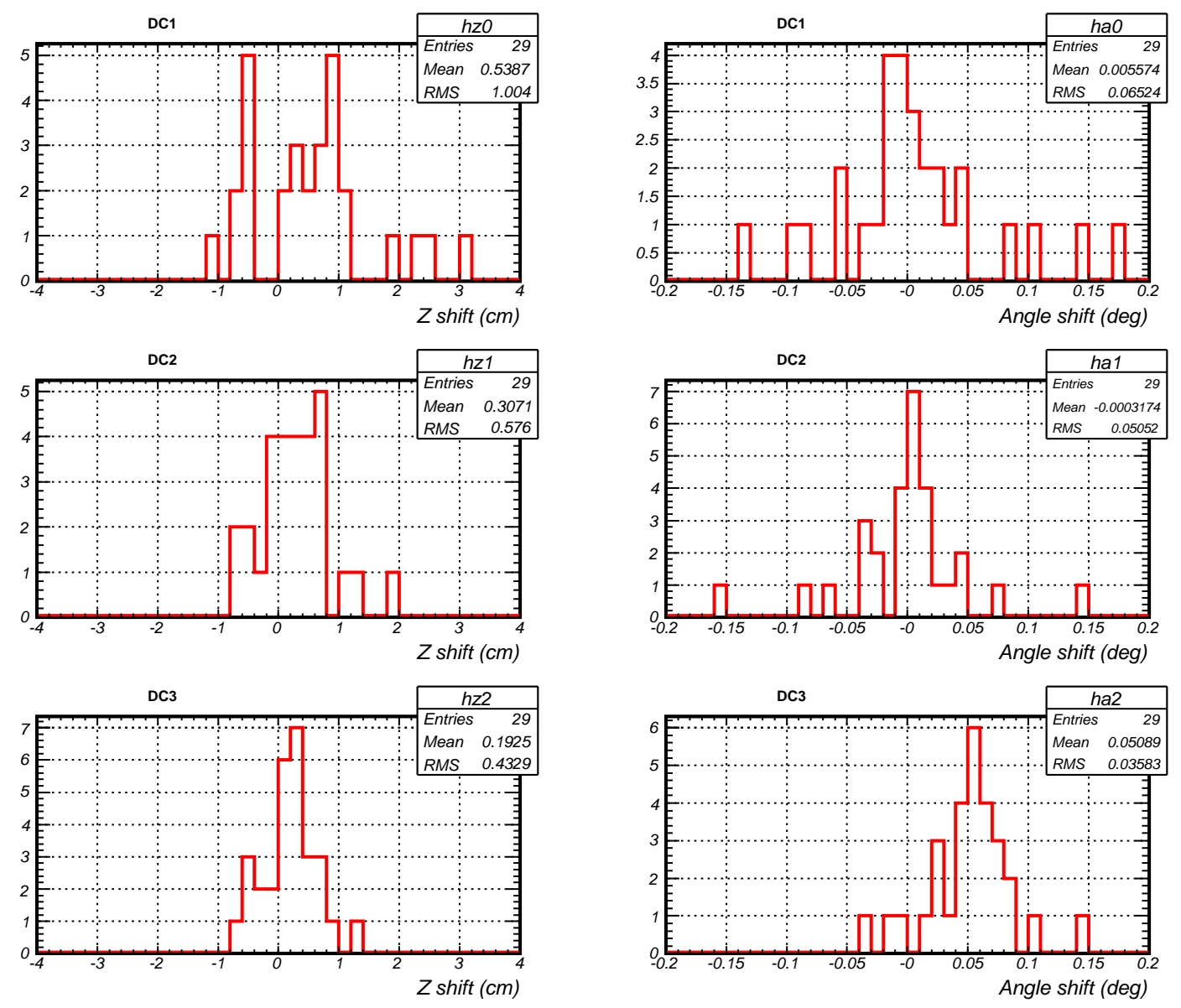

Figure 7.7: DC123 z-alignment with field ${ }_{\lambda}$ off runs with no known geometry errors. Still, DC3 is being systematically rotated by $0.055^{\circ}$ by $z$-alignment algorithm in 29 different runs from 5 different time periods. 

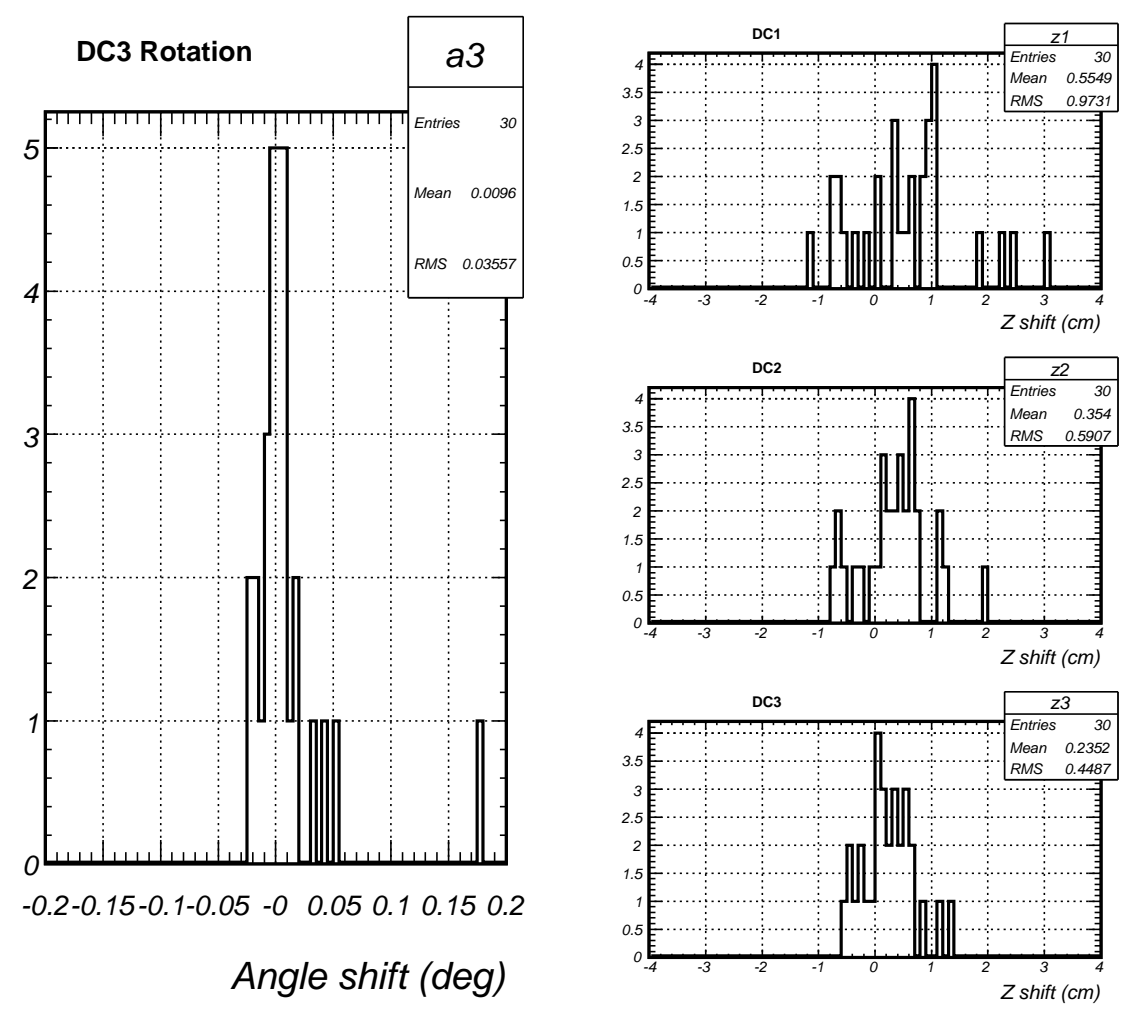

Figure 7.8: DC123 z-alignment after rotation in DC3 was removed. 
respectively. These corrections were not applied for the following reasons:

- We cannot easily estimate effect from Earth's magnetic field and residual field in the magnets;

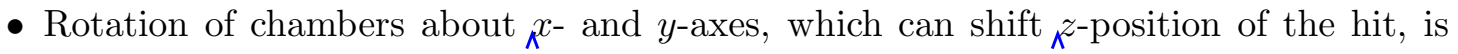
not taken into account by reconstruction;

- The systematic error on position measurement due to these misalignments is less than 0.1 wire spacingt.

\subsection{Tuning ${ }_{\Lambda}$ Magnetic Field}

Measurement of magnetic field $d_{\Lambda}$ with Ziptrack had very small uncertainties in positions of the Hall probe jig, but due to the size of the holder, precise rotation of the probe could not be set or measured. This leaves room for dominant component to bleed

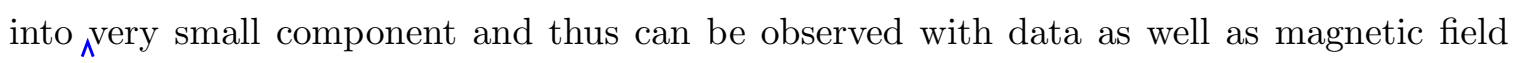
measurements.

\subsection{1 $B_{x} / B_{y}$ Rotation}

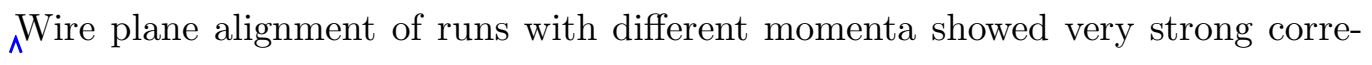
lation of alignment constant for vertical-measuring planes of the PWCs (see Figures 7.9 and 7.10). The most likely possibility for this problem was large ${ }_{\wedge}{ }_{x}$ component of the field in magnet field map.

To determine whether the dominant $B_{y}$ bleeding into $B_{x}$ is responsible for this correlation, we ran another track $\chi^{2}$ minimization algorithm, where only 9-chamber beam tracks were used, and the algorithm was free to rotate $B_{x}$ and $B_{y}$ components:

$$
\begin{aligned}
& B_{x}^{\prime}=B_{x} \cos \theta+B_{y} \sin \theta, \\
& B_{y}^{\prime}=-B_{x} \sin \theta+B_{y} \cos \theta .
\end{aligned}
$$

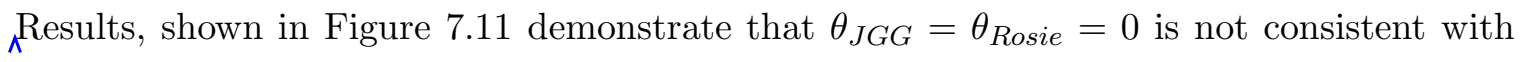

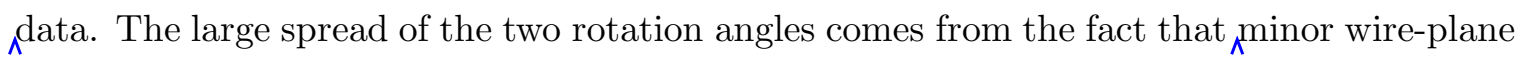
misalignment will have an enormous effect on large-momentum runs, where the minimizer will compensate for the misalignment by inflating $B_{x}$. 

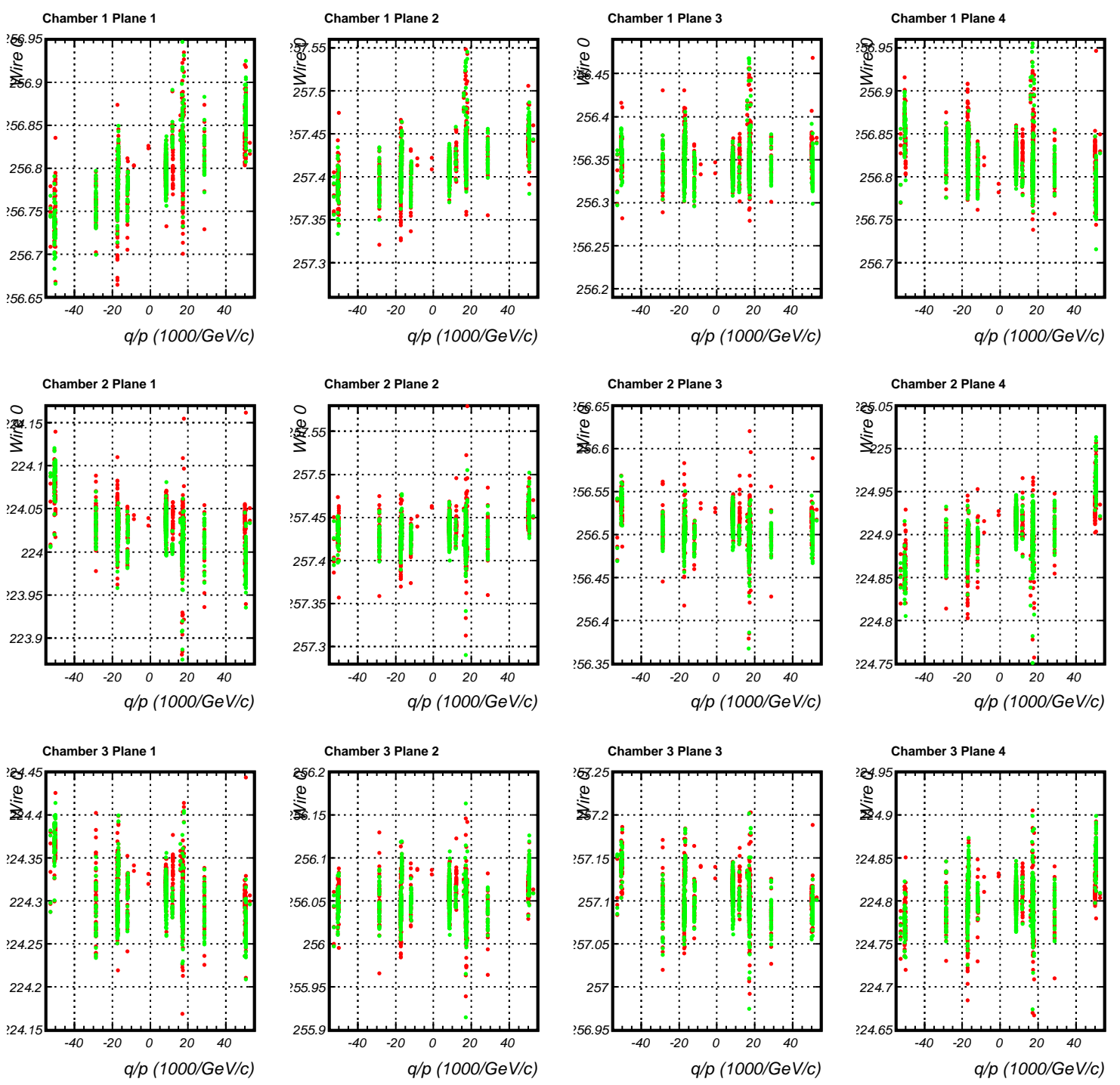

Figure 7.9: DC123 alignment results for runs with different beam momenta. Red dots come from alignment with ${ }_{\wedge} \mathrm{BCs}$ fixed, and green diamonds from alignment with ${ }_{\wedge}^{\mathrm{BCs}}$ allowed to vary. 

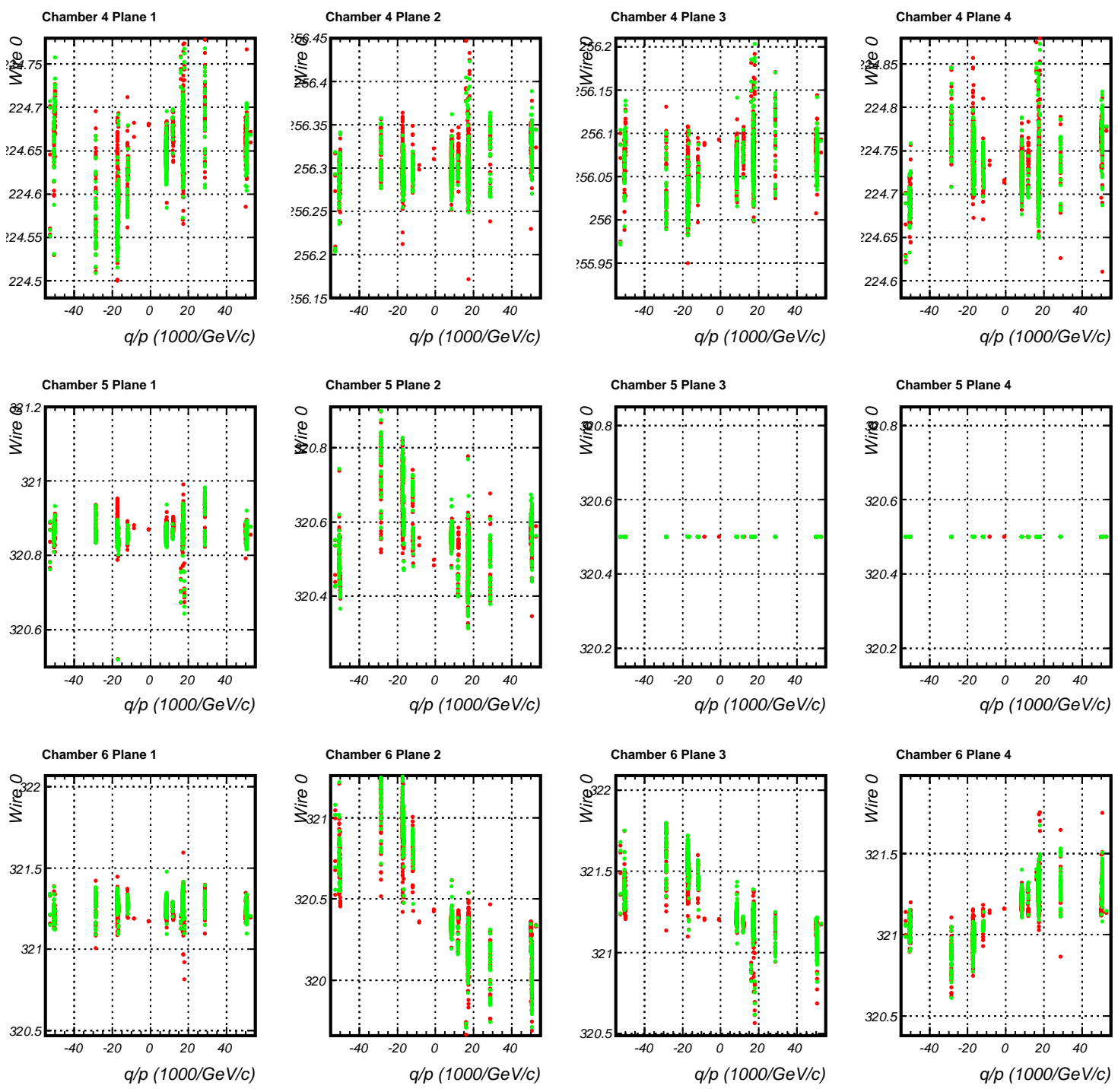

Figure 7.10: DC4 and PWC56 alignment results for runs with different beam momenta. Red dots come from alignment with ${ }_{\Lambda} \mathrm{BCs}$ fixed, and green diamonds from alignment with ${ }_{\wedge}^{\mathrm{BCs}}$ allowed to vary. Notice the large correlations of alignment offset in the second (verticalmeasuring) plane of PWC5 and PWC6. 


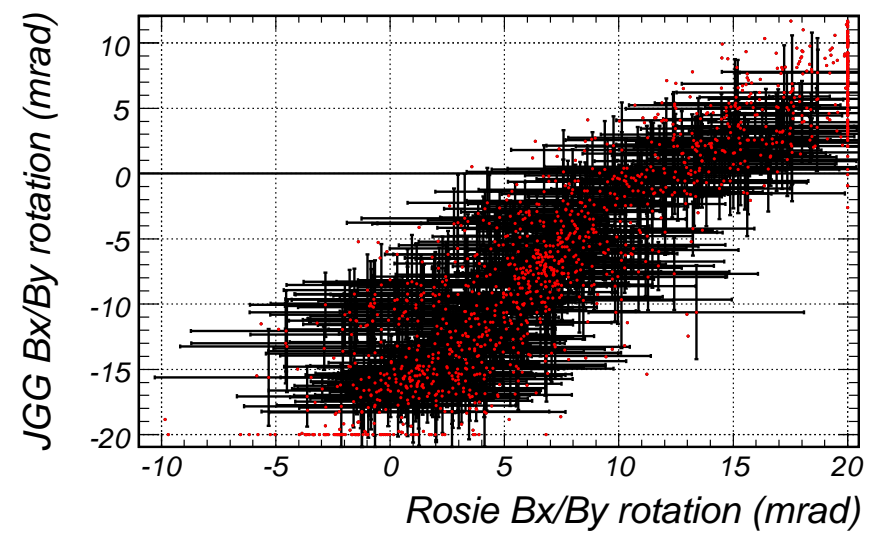

Figure 7.11: Results of optimizing $B_{x} / B_{y}$ rotation. While the different runs are clustered in a line rather than a single point, all runs exclude rotation $(0,0)$ for the two magnets' field components.

The two magnets' fields were measured with the same apparatus rotated by $180^{\circ}$ about $y$-axis. Therefore, if the misalignment truly comes from rotation of the Ziptrack Hall probe holder, the $B_{x} / B_{y}$ ratio in the center of the magnet (where $B_{x}$ has to be vanishingly small), must be equal and opposite. Figure 7.12 clearly shows that in the case of $B_{x}$, the ratio is about $0.7 \%$, and in the case of $B_{y}$, the ratio is $0.3 \%$. The point $(7,-7)$ is supported by the data, as it falls in the middle of the line in Figure 7.11, so both magnets' field map $B_{x} / B_{y}$ components were rotated by $7 \mathrm{mrad}$ in opposite directions.
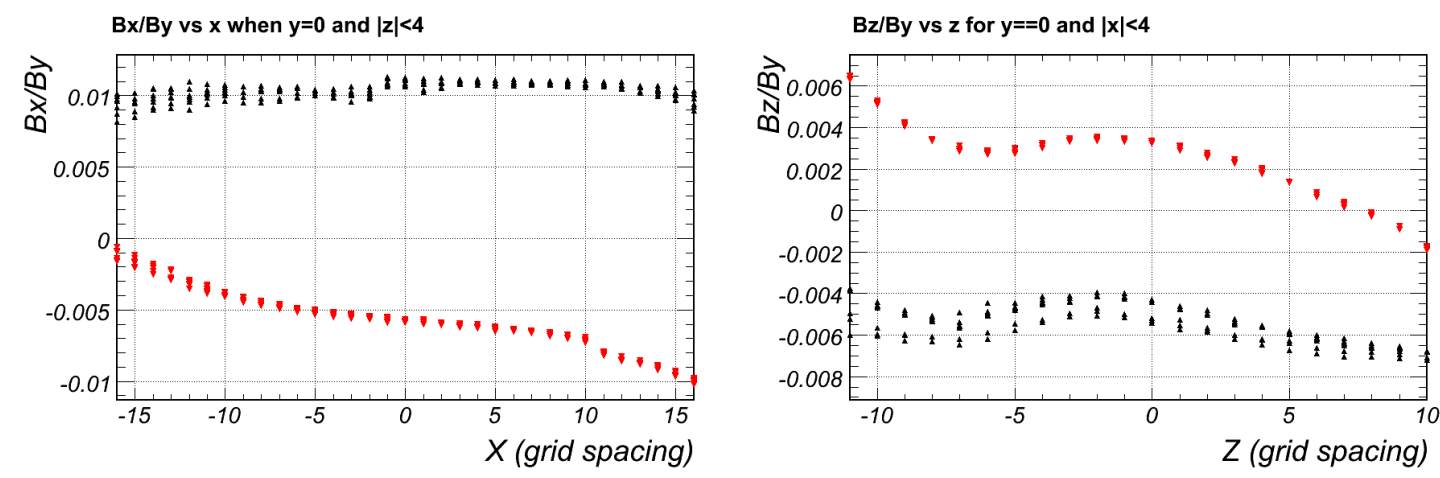

Figure 7.12: The ratios of field components in the center of the magnet. JGG is shown in up-pointing black triangles and Rosie in downward-pointing red triangles. 


\subsection{2 $B_{y} / B_{z}$ Rotation}

While the experiment is not very sensitive to $B_{z}$ since most track angles are small with respect to the $z$-axis, the Hall probe measurements do suggest a 3 mrad rotation of ${ }_{\wedge} B_{z} / B_{y}$ components, which was done. The summary of chamber wire plane alignment constants with $x y$ and $y z$ rotations taken into account is shown in Figures 7.13 and 7.14.

\subsection{3 $B_{x} / B_{z}$ Rotation}

The most difficult rotation of magnetic field components to correct is that about $\Lambda^{y}$-axis. $B_{x}$ and $B_{z}$ are comparable in most regions of the magnet, so $B_{x} / B_{z}$ or $B_{z} / B_{x}$ ratios of magnetic field measurements are not as easy to interpret as in the case of ${ }_{\Lambda} B_{y} / B_{z}$ ratio. While tracking is not very sensitive to this rotation, ${ }_{\Lambda}$ PPC drift correction is. To determine the optimal rotation, the slope of ${ }_{\Lambda}$ PC $x$-residual vs $z$ was measured for a different values of the component rotation. Results shown in Figure 7.15 suggest that the data wants to have the components rotated by $9.6 \mathrm{mrad}$.

The ratio of magnetic field components favors rotation of the same magnitude (see Figure 7.16) to achieve better symmetry of the $B_{x} / B_{z}$ ratio. Given that rotation is of the same order of magnitude as the other two rotations, and ${ }_{\Lambda} \mathrm{TPC}$ is more sensitive to it than the magnetic measurements are, rotation of $9.6 \mathrm{mrad}$ was applied to both JGG and Rosie.

\subsection{TPC Electron Drift}

Understanding of electron drift in the TPC consists of two more or less independent problems. The first is measuring drift velocity in the absence of magnetic field ${ }_{\Lambda}$ which is equal to drift velocity when magnetic and electric fields are collinear. We call this velocity $v_{0}$. The second is drift velocity components given magnetic field and the angle between magnetic and electric fields (electric field is assumed to be constant $122 \mathrm{~V} / \mathrm{m}$ ). This 3-dimensional effect distorts the tracks from straight-line projection in a complicated fashion by as much as $8 \mathrm{~cm}$ in $x$ and $10 \mathrm{~cm}$ in $z$. Good understanding of this effect is crucial if TPC measurements are to be trusted. 


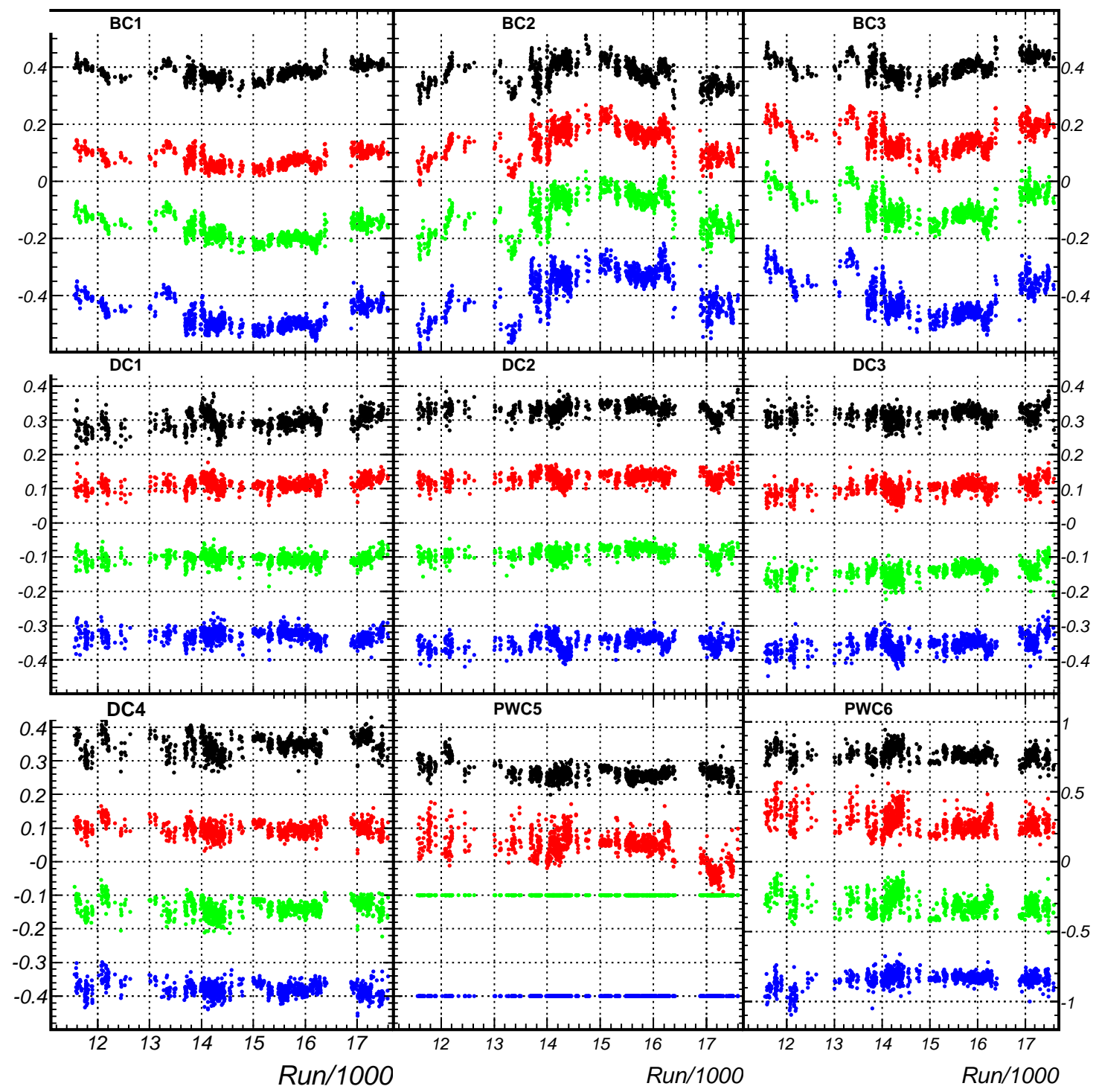

Figure 7.13: Alignment summary with geometry and magnetic field corrections. Plane 1 is at the top of every set of graphs for each chamber. Note: the $y$-scale of PWC6 plots is different from all other chambers. 


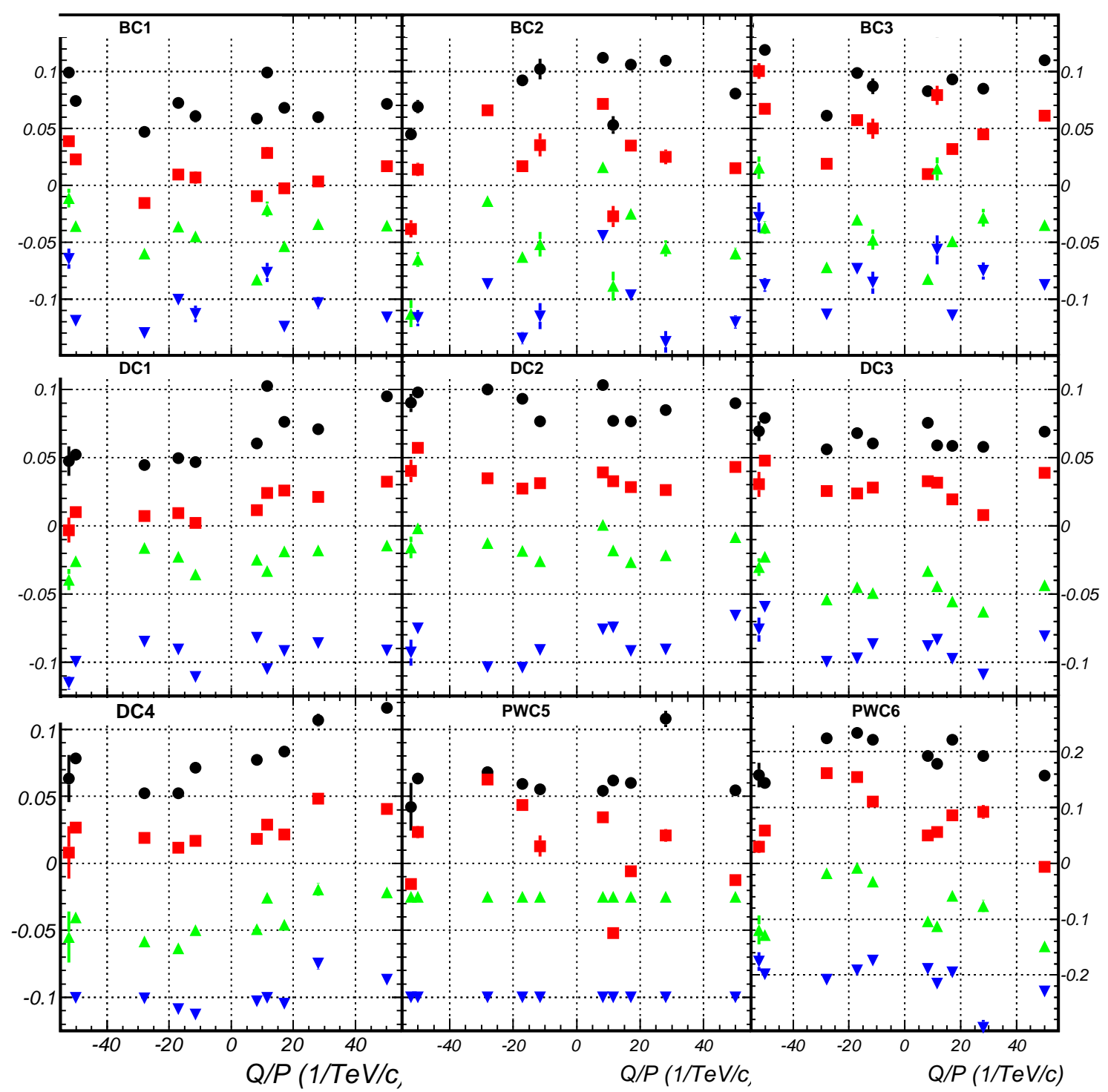

Figure 7.14: Alignment summary with geometry and magnetic field corrections. Plane 1 is at the top of every set of graphs for each chamber. While correlations with momentum still exist, they are small, and their effect on tracking can be measured. Note: the scale of PWC6 plots is different from all other chambers. 

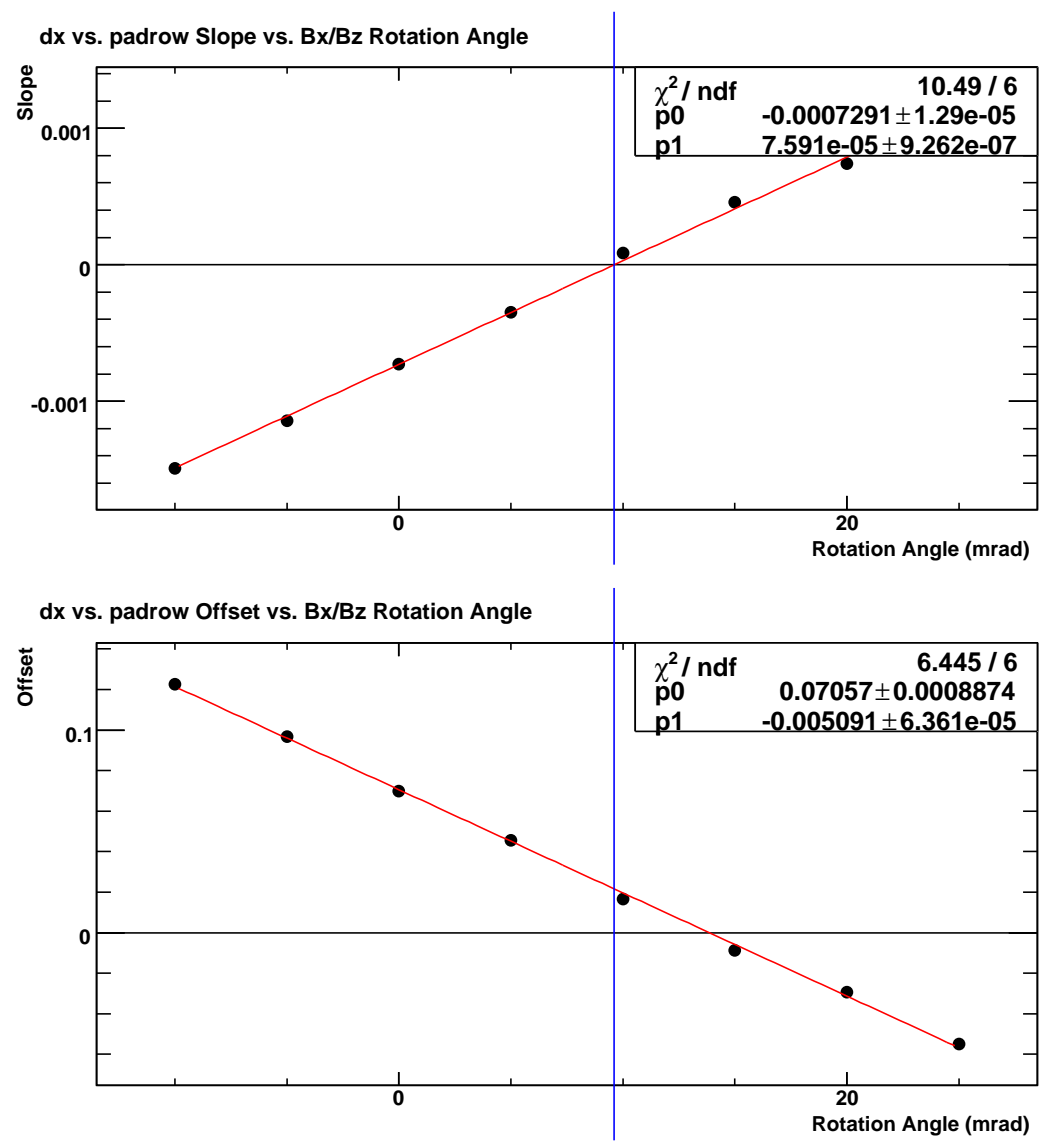

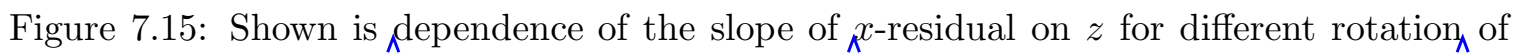
$B_{x} / B_{z}$ components. 

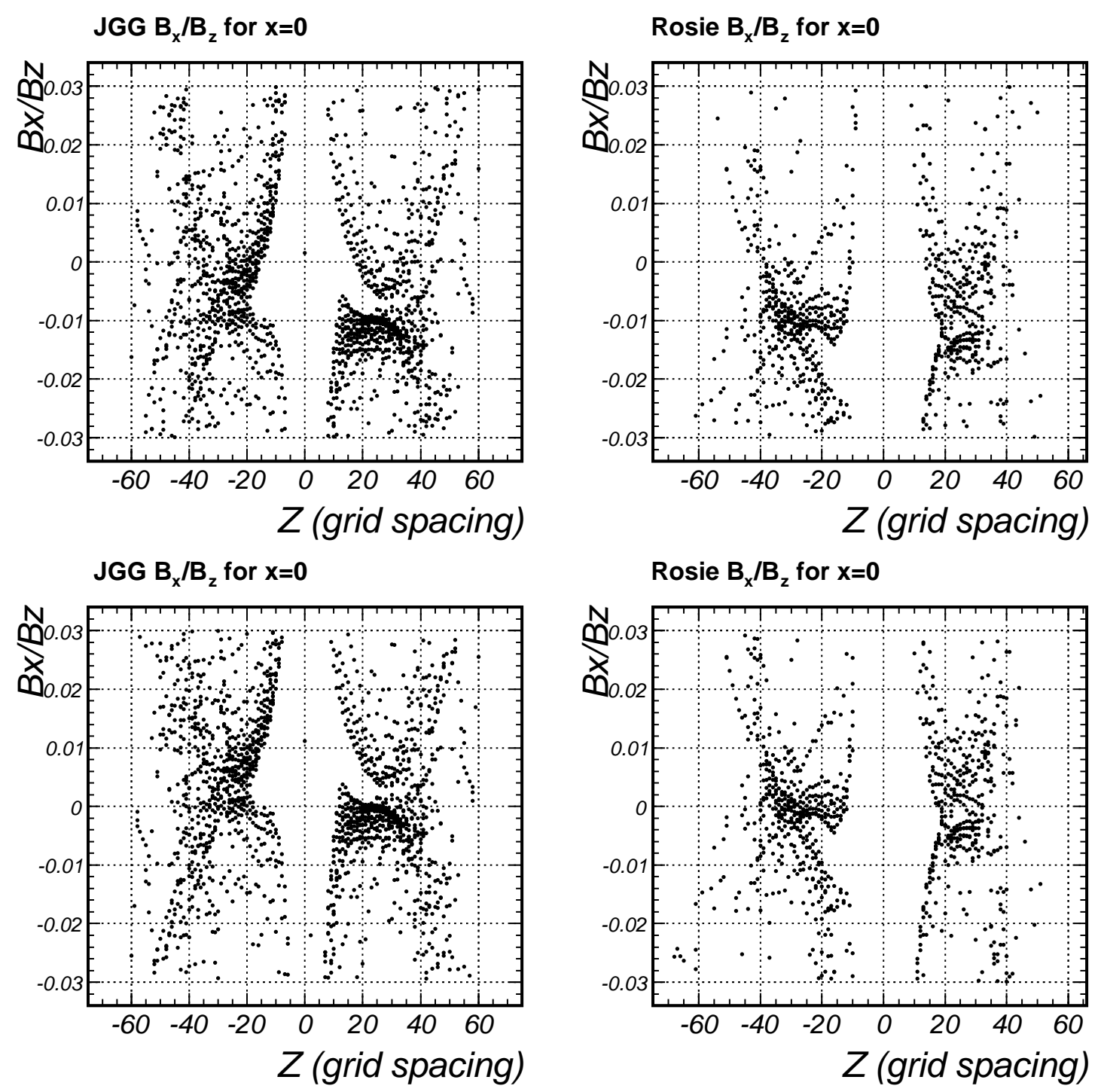

\section{Rosie $B_{x} / B_{z}$ for $x=0$}

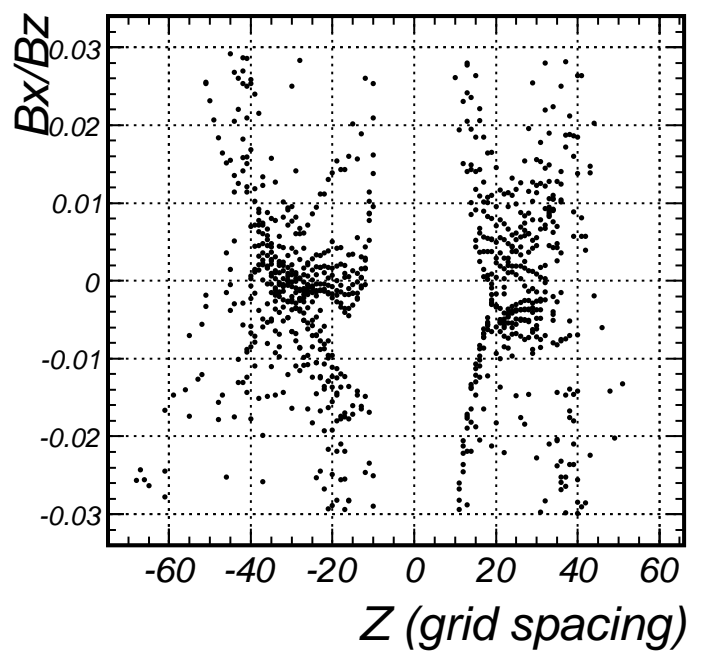

Figure 7.16: The top pair of plots shows the ratio of $B_{x} / B_{z}$ of the two magnets for $x=0$ in the field map. The bottom pair shows the same ratio when the field components are rotated by $9.6 \mathrm{mrad}$. Note that unlike $B_{x} / B_{y}$ and $B_{z} / B_{y}$, the sign of this ratio is expected to be the same for both magnets, since the Hall probes were rotated about $y$-axis. 

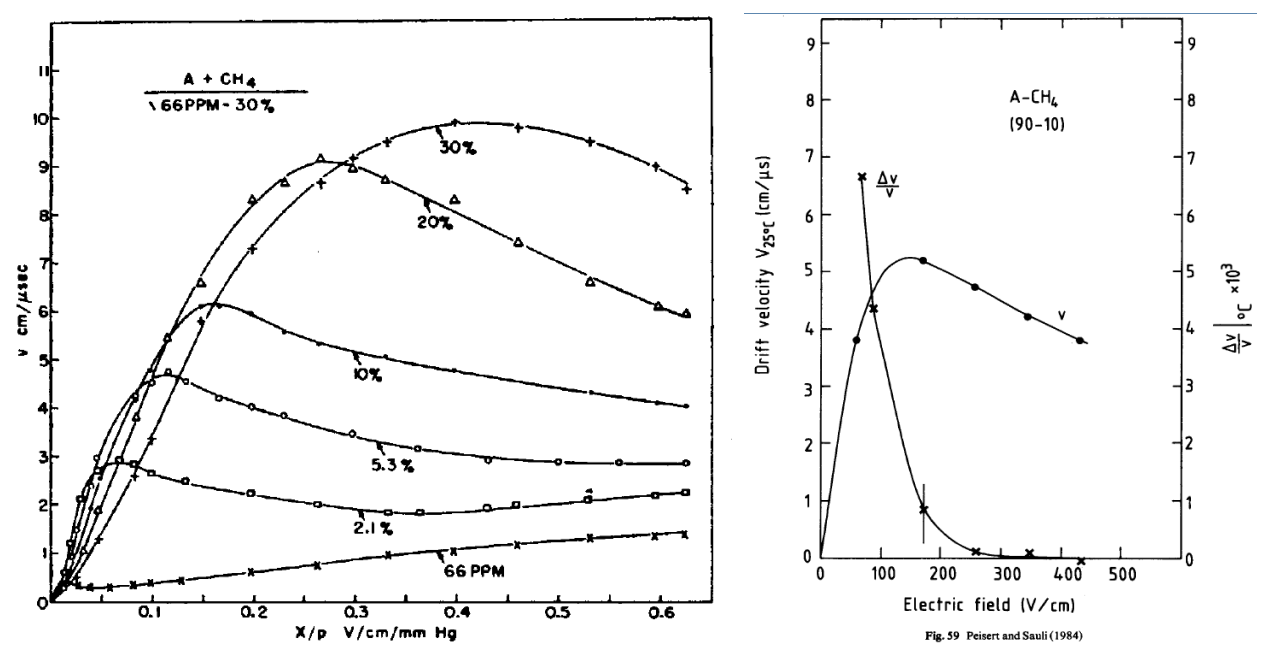

Figure 7.17: Two sets of measurements of drift velocity in P10 mixture[14, 37].

\subsubsection{Measuring $v_{0}$}

${ }_{\lambda}$ Drift velocity in argon-methane mixtures is a function that depends on a number

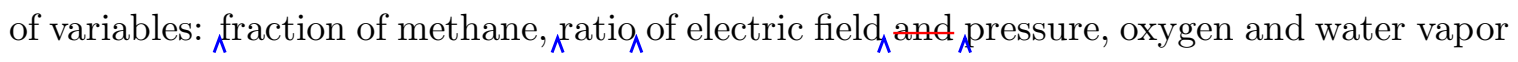
contamination, and gas temperature. Measurements for clean P10 (90\% Ar 10\% $\left.\mathrm{CH}_{4}\right)$ are shown in Figure 7.17. The choice ${ }_{\Lambda}^{\mathrm{P} 10}$ at atmospheric pressure makes dependence of drift velocity on pressure very small when electric field is about $120-150 \mathrm{~V} / \mathrm{cm}$.

Distortions of the drift velocity in the center of the TPC are small as the field is quite uniform. This makes it possible to measure drift velocity even when magnetic field is on using reconstructed hits in the center of the TPC drift volume.

In order to remove any sort of bias from measurement of the drift velocity, we did full reconstruction of event, and then refit the vertex using information from chambers only. In order to achieve this using one value of drift velocity for all runs, the cut windows to match $\wedge^{T P C}$ track to chambers were quite loose. Once the vertex-constrained fit was done, we selected vertices with three or more tracks, and saved position predicted at the $z$ of the hit and its measured time. One can take two distinct approaches to measure velocity: bin the data in $t$ or $z$ and do a least squared fit or do a non-linear fitting where the square of distance from the straight line to the data point is minimized. The latter method has the advantage that outliers have a smaller effect on the fitted slope, and therefore is not as sensitive to event selection as the former method. 


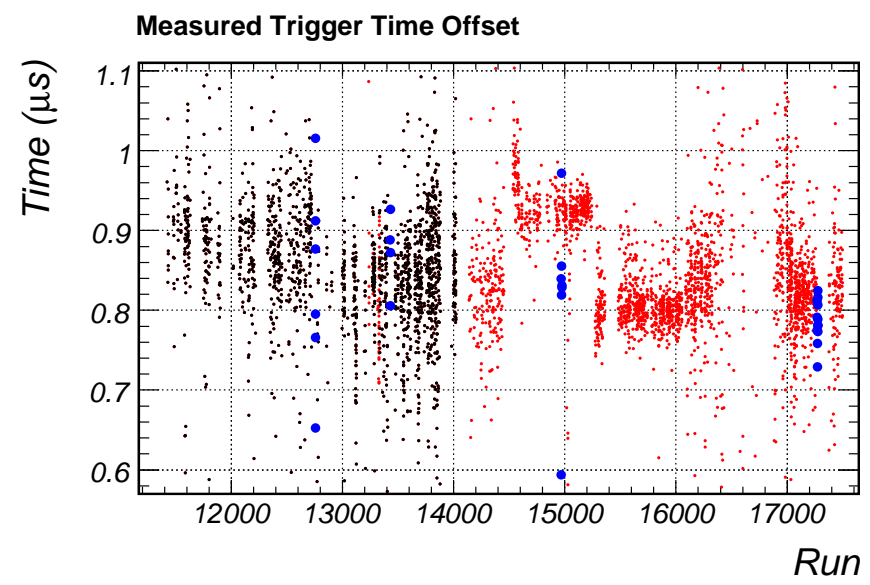

Figure 7.18: Measured TPC trigger delay time as a function of run. Large blue circles come from field off runs. See text for further explanation.

One of the unknowns in the system is trigger delay time ${ }_{\Lambda}$ which should be constant from run to run. Figure 7.18 shows the measured trigger offset as a function of run. There are some notable features in the plot:

- Runs before 12700 have a systematically different trigger delay. Between runs 12700 and 13000, a lot of work on the trigger was done in the hall, so while we do not know how much the trigger time was changed, the data suggests a 70 ns difference.

- NuMI runs (14500-15250) measure a different trigger delay. This is not very surprising, because NuMI events are very busy and therefore wide windows to match TPC tracks to chamber data may have an adverse effect.

- Field $\mathrm{\lambda}_{\Lambda}$ off data gives approximately the same delay as field $\mathrm{\lambda}_{\lambda}$ on data, which is a confirmation that the effect of the magnetic field on the drift velocity in the center of the TPC is negligible.

- Runs before 14100 were taken with a larger gating grid delay, which limited the active volume of the TPC. Reduction of the gating grid delay did not affect trigger delay.

With these results, we fix the delay to $875 \mathrm{~ns}$ for runs before 12700 and to $800 \mathrm{~ns}$ for runs above 12700. Then we go back to the data to measure drift velocity with constant trigger delay. Figure 7.19 shows the measured drift velocity when trigger offset is free, and when it is fixed. 


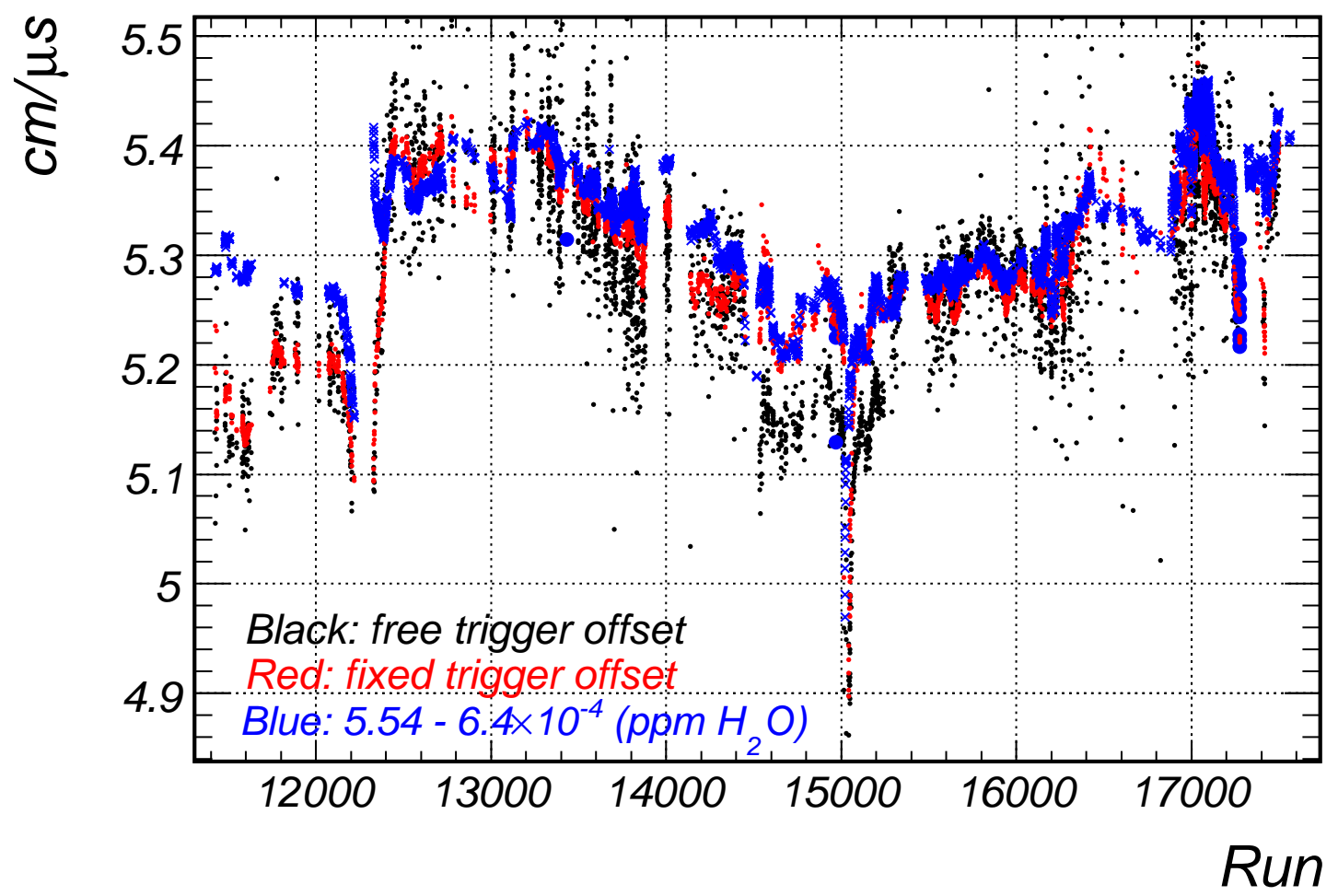

Figure 7.19: Measured TPC drift velocity as a function of run. The benefit of fixing trigger time is clear: the precision of velocity measurement is much higher, and the correlation with water content in the TPC gas becomes much stronger. 


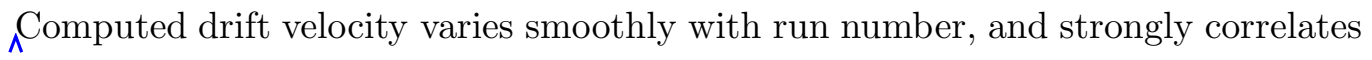
with water content measured in the TPC exhaust gas. Therefore, we feel that $v_{0}$ is measured to $0.01 \mathrm{~cm} / \mu \mathrm{s}$, or $0.2 \%$. The uncertainty of $10 \mathrm{~ns}$ on $\lambda_{\Lambda}$ rigger delay time adds $\lambda_{\Lambda} .2 \%$ systematic error on hit position. At the top of the $80 \mathrm{~cm}$ drift volume, this translates into precision of $\Lambda^{y}$-measurement of better than $2.4 \mathrm{~mm}$.

\subsubsection{Distortion Corrections}

Electron drift in a non-uniform magnetic field is a complicated problem. Conceptually, it is clear that if electric and magnetic fields are not collinear, the force due to magnetic field on electron creates a force proportional to $\vec{E} \times \vec{B}$, but electron motion in that direction will cause additional force proportional to $(\vec{E} \times \vec{B}) \times \vec{B}$, hence all three components of the drift velocity are non-zero.

A linear model of drift is motivated by $\lambda_{\Lambda}$ drag force $\lambda_{\Lambda}$ which is proportional to $\Lambda^{\text {drift }}$ velocity:

$$
\frac{d \vec{v}}{d t}=\frac{e}{m} \vec{E}+\frac{e}{m} \vec{v} \times \vec{B}-\frac{\vec{v}}{\tau}=0
$$

where $\vec{v}$ is the drift velocity, $e$ is electron charge, $m$ its mass, $\vec{E}$ is the electric field, $\vec{B}$ is the magnetic field and $\tau$ can be though of as the mean time between collisions of drifting electron and gas molecules. Equation 7.2 can be solved to obtain

$$
\vec{v}=-\frac{|e| \tau E / m}{1+b^{2}}\left(\begin{array}{c}
\left(1+b_{x}^{2}\right) e_{x}+\left(-b_{z}+b_{x} b_{y}\right) e_{y}+\left(b_{y}+b_{x} b_{z}\right) e_{z} \\
\left(b_{z}+b_{x} b_{y}\right) e_{x}+\left(1+b_{y}^{2}\right) e_{y}+\left(-b_{x}+b_{y} b_{z}\right) e_{z} \\
\left(-b_{y}+b_{x} b_{z}\right) e_{x}+\left(b_{x}+b_{y} b_{z}\right) e_{y}+\left(1+b_{z}^{2}\right) e_{z}
\end{array}\right)
$$

where signs take into account the negative charge of electron, $\vec{E}=E\left(e_{x}, e_{y}, e_{z}\right), v_{0}=$ $|e| \tau E / m$, and $b_{i}=\frac{|e| \tau}{m} B_{i}=\frac{v_{0}}{E} B_{i}$.

If $\vec{E}=E \hat{y}$, and $\vec{B}=B(\cos \theta, \sin \theta, 0)$, this equation reduces to:

$$
\vec{v}=-\frac{v_{0}}{1+b^{2}}\left(\begin{array}{c}
b^{2} \cos \theta \sin \theta \\
1+b^{2} \cos ^{2} \theta \\
b \sin \theta
\end{array}\right)
$$

where $b=v_{0} B / E$.

However, when this equation is used to correct drift, one quickly sees that there is significant over-correction in both $x$ and $y$ (Figure 7.20). While we do not know the reason why linear model fails to describe the drift in the TPC, the solution was to use Magboltz 

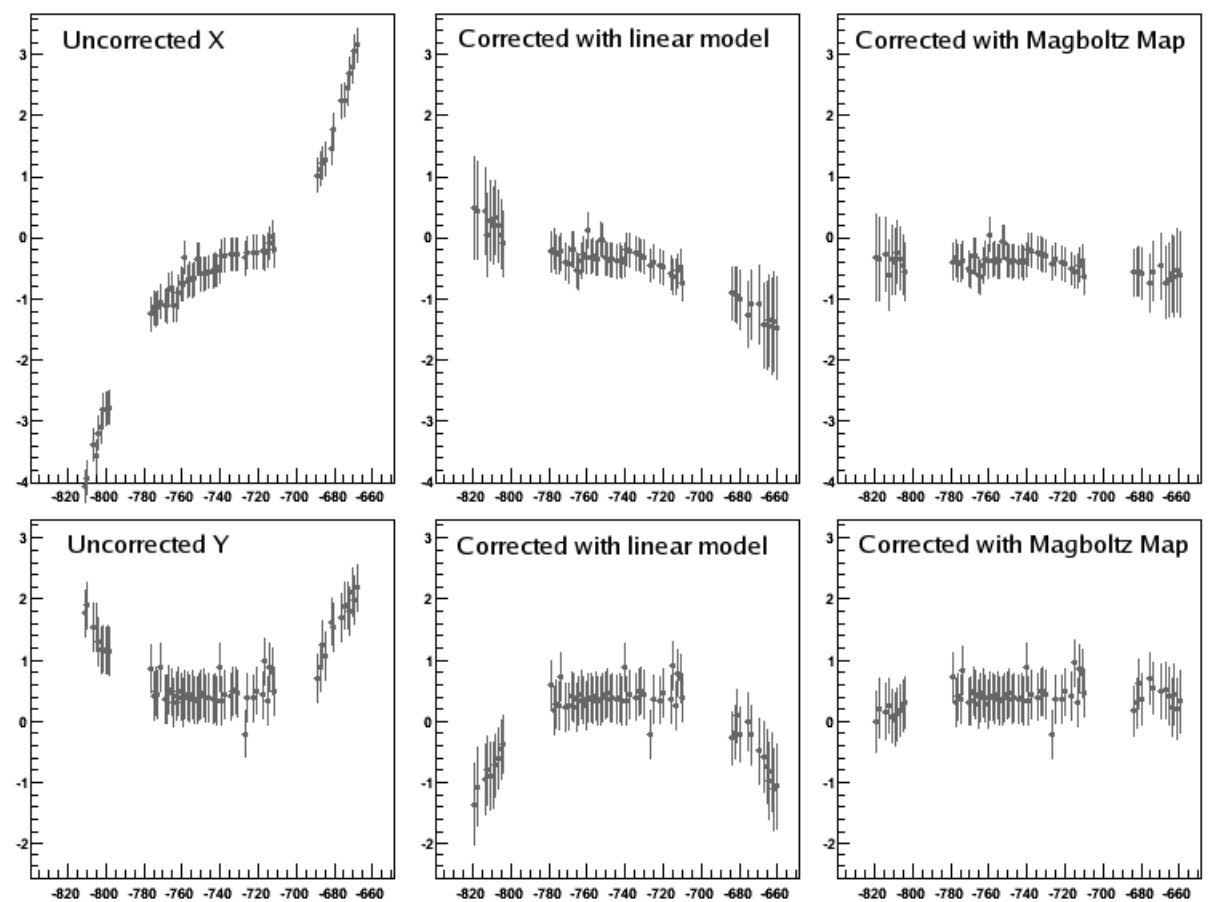

Figure 7.20: Shown are $x z$ and $y z$ projections of TPC hits from a $120 \mathrm{GeV} / c$ track. The units on both axes are $\mathrm{cm}$. 


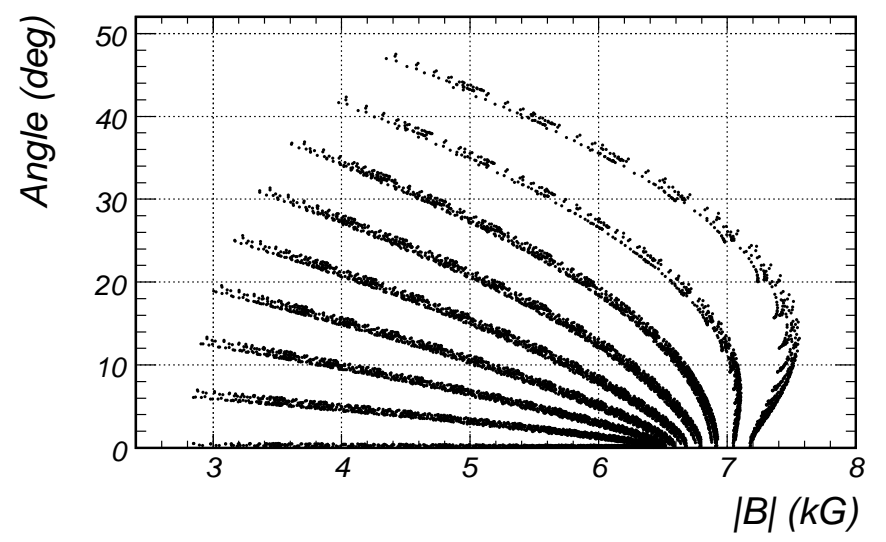

Figure 7.21: Angle between the vertical and horizontal field components in the JGG field map inside the TPC drift volume.

Monte Carlo program[38, 39]. Magboltz takes the gas mixture, electric field, and constant magnetic field at arbitrary angle with respect to the electric field. Simulated trajectories of $10^{6}$ electrons are used to predict the three drift velocity components. In order to use results given by Magboltz, we had to create a map of drift velocity components in $(B, \theta)$ space, with $\theta$ is defined by Equation 7.4. Figure 7.21 shows that we needed to model angles up to $50^{\circ}$.

We included all details of the gas mixture that was provided by our monitoring:

- Oxygen concentration, which was relatively stable at $350 \mathrm{ppm}$.

- Nitrogen concentration at 78/21 of oxygen concentration, since oxygen must have come from air.

- Water concentration. Since drift velocity strongly correlates with water content, gas mixtures with 50, 300, 550, 700, and $950 \mathrm{ppm}$ of water were modeled with $v_{0}$ of 5.403 , $5.299,5.179,5.097$, and $4.965 \mathrm{~cm} / \mu$ s respectively.

Equipped with 5 Magboltz maps and $v_{0}$ for a run, we compute the map for the run using a weighted sum of the 2 maps with $\Lambda_{\Lambda}$ losest $v_{0}$, since $v_{i}(B, \theta) / v_{0}$ depends on $v_{0}$. As Figure 7.20 shows this model of electron drift agrees with the data. 


\subsection{RICH $R_{0}$}

Over the course of the run, ${ }_{\Lambda} \mathrm{CO}_{2}$ density in the $\mathrm{RICH}$ fluctuated by about $1.5 \%$, which translates into a $3 \mathrm{~mm}$ difference in $R_{0}$ ( $\beta=1$ ring radius). The RMS width of ${ }_{\Lambda} 120 \mathrm{GeV} / c$ proton ring distribution is about $1.8 \mathrm{~mm}$, so at high momenta density fluctuations widen the ring radius distribution if ring radius is left uncorrected.

In the case of $120 \mathrm{GeV} / c$ thin target data, calibration of the effective index of refraction is easily achieved by measuring radii of uninteracted protons. In a typical file with 5000 events one finds more than 1000 clean events with uninteracted protons. We can rewrite Equation 6.25 as

$$
\frac{1}{2} R_{0}^{2}=F^{2}-\frac{F^{2}-\frac{1}{2} R^{2}}{\sqrt{1+m_{\text {prot }}^{2} / p_{\text {beam }}^{2}}},
$$

which allows us to compute $R_{0}$ from measured proton ring radius. Figure 7.22 shows the summary of RICH calibration thus obtained. 

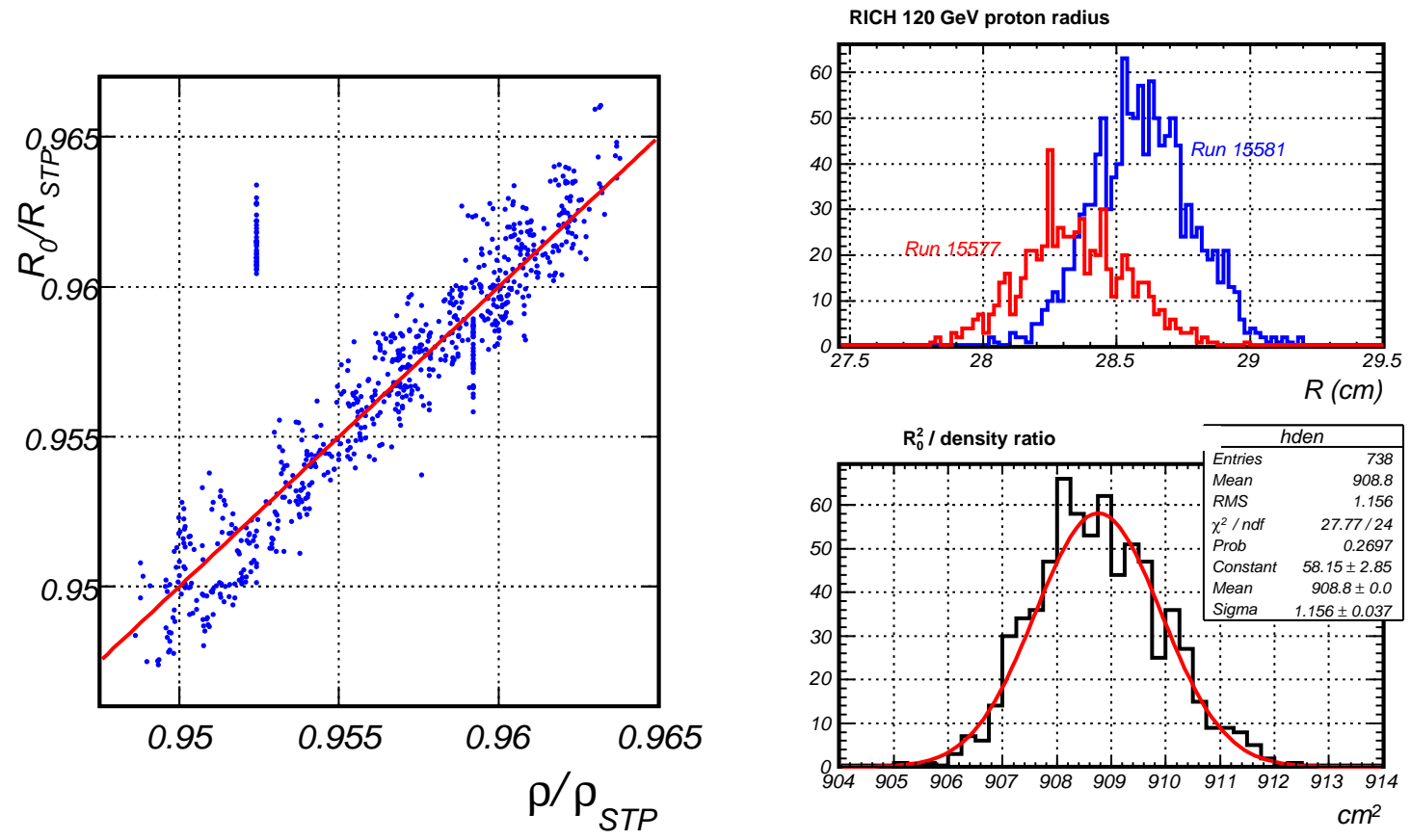

Figure 7.22: The left plot shows dependence of $\mathrm{RICH} R_{0}^{2}$ on the density of $\mathrm{CO}_{2}$, where both variables are divided by respective value at STP. The few outliers come from subruns where gas temperature or pressure are not recorded in the database. The $y=x$ line is superimposed to demonstrate that the expected linear dependence between $R_{0}^{2}$ and gas density is observed. The top right plot shows the difference in proton ring radius distributions in two extreme nearby runs. ${ }_{\Lambda} \mathrm{CO}_{2}$ pressure was raised between the two runs. Bottom right plot tells us that $R_{0}^{2}$ is known to about $0.1 \%$. 


\section{Chapter 8}

\section{Monte Carlo Simulation}

Monte Carlo simulation was necessary in order to measure reconstruction performance. In particular for this analysis, we need to know particle identification efficiency and backgrounds.

We used FLUKA-06 [23, 24] to simulate particle production and GEANT 3.21 [9] to transport particles through the spectrometer. GEANT hits (idealized energy deposition) were stored into the ROOT event tree, and hits were converted into digits (mock raw data) with packages written by the MIPP collaboration.

\subsection{Time Projection Chamber Digitization}

In order to get an accurate model of the TPC track, the following effects have to be modeled:

- Electron drifting in non-uniform magnetic field,

- Variations in gas gain due to different anode voltages,

- Diffusion of charge in space,

- Diffusion of charge in time,

- Electronic noise.

${ }_{\Lambda}^{T P C}$ gas volume inside the field cage has dimensions of $104.4 \times 79.4 \times 162.8 \mathrm{~cm}$. It was was segmented into $0.87 \times 1.05 \times 1.27 \mathrm{~cm}$ rectangular parallelepipeds so that GEANT 
could record hits at boundary crossings. The objective of digitization is to compute the $\mathrm{ADC}$ in each voxel. A voxel is defined as time slice on a pad in a padrow.

\subsubsection{Generating Digits}

Starting from hit position $(x, y, z)$, we drift the hit to anode wire plane obtaining $\left(x^{\prime}, y_{\text {anode }}, z^{\prime}\right)$ and drift time $t_{d}$ (see Section 7.5.2). ${ }_{\wedge}$ Fourth $h_{\wedge}$ order Runge-Kutta stepper was implemented to do this, and with $0.5 \mu$ s steps it computed distortion with precision of about 1 micron, as tested by reversing the drift to restore input position.

Before TPC digits are generated, $\left(x^{\prime}, z^{\prime}, t_{\text {drift }}\right)$ are converted to pad column, padrow, and time bucket $\left(x_{c o l}, x_{\text {row }}, t_{b k t}\right)$ through

$$
\begin{aligned}
x_{\text {col }} & =\left(x^{\prime}-x_{c o l, 0}\right) / L_{c o l} \\
x_{\text {row }} & =\left(z^{\prime}-x_{\text {row }, 0}\right) / L_{\text {row }} \\
t_{\text {bkt }} & =\left(t_{d}-t_{\text {trig }}\right) / T_{b k t}
\end{aligned}
$$

Here $x_{c o l, 0}$ and $x_{r o w, 0}$ are locations of column 0 and pad $0, L_{c o l}=0.8 \mathrm{~cm}$ and $L_{\text {row }}=1.2 \mathrm{~cm}$ are pad sizes, and $T_{b k t}=0.1 \mu \mathrm{s} /$ bucket. Trigger time $t_{\text {trig }}$ is generated as a constant time

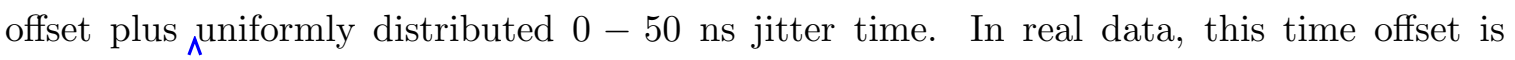
measured, and the jitter results from the fact that the TPC $20 \mathrm{MHz}$ clock is not synchronized to trigger time.

The next step is to spread the hit energy deposition over pads, columns and buckets. To improve performance of the algorithm, we defined a 3-dimensional array of total charge in TPC voxels. At the beginning of event, charge on each voxel is reset to 0 . For each hit, we define a three dimensional energy density function as the product of Gaussian in pad column centered at $x_{c o l}$, Gaussian in padrow centered at $x_{\text {row }}$, and pamma function in buckets with the peak at $t_{b k t}$. The integral of the the function is set to hit energy deposition multiplied by gain factor ${ }_{\wedge}$ which is different for every anode section of the TPC. For each TPC voxel where the integral of the density function is at least 0.5 counts, the charge sum is incremented, and the track number that generated the hit is added to the track list associated with the voxel. Having the associated track list allows to establish correspondence between ${ }_{\wedge}$ reconstructed track and MC track.

In the course of the run, some anode regions were not able to hold the nominal high voltage of $1250 \mathrm{~V}$, thus creating large dead regions in the TPC. Undoubtedly, the missing 
data has an adverse effect on track finding algorithm, so it has to be modeled. Each anode region was assigned a different gain: the factor converting ${ }_{\Lambda}$ GEANT hit energy deposition to $\mathrm{AD}_{\Lambda} \mathrm{C}$. Additional missing data comes from electronic problems as a number of sticks (two half-padrows) never readout. These sticks were turned off in the Monte Carlo simulation.

To generate TPC digits, for each voxel on a good stick with at least 1 ADC count, we multiply the charge by $a_{\lambda}$ Gaussian random number centered at 1 to randomize pad gain. Finally, we add a Gaussian pedestal and if the total charge in the voxel is above threshold, it is written out.

\subsubsection{Generating Noise}

There are 3 sources of noise in the TPC:

1. Hot voxels;

2. Hot pads where all voxels are lit up or every other voxel is on;

3. Charged cosmic particles.

All of these sources of noise are typically well rejected by the TPC reconstruction alogrithm. Cosmic particles are rare, and are not likely to go through the target, so they were not modeled. In order to speed up modeling of noise, a random Gaussian number of voxels was selected to be noisy in an event and their ADC spectrum was was generated to be consistent with data.

\subsection{Wire Chamber Digitization}

GEANT hits in wire chamber planes were converted to hit wires by computing the number of drift cells that the track crossed, and dividing $\Lambda_{\Lambda}$ deposited energy equally between all wires. A track number list is filled for every wire as the deposited energy is being added to wires. Charge from all hits is integrated over all wires, and wires above threshold are written out as digits. This approach makes it more difficult to tune $\boldsymbol{\Lambda}_{\Lambda}$ chamber efficiency than choosing a random number for each wire. However, it is motivated by the physics of wire chambers, and makes it possible to simulate cross talk where it is most likely to happen given distribution of particles at the chamber. 
In addition to computing wires above threshold, drift time needs to be calculated in the drift chambers. As the charge is being split between wires, the minimum distance from track to wire is stored. In the case where more than 2 drift cells are crossed, minimum distance is set to 0 for the inner wires. Minimum distance is converted into drift time using $\Lambda_{\Lambda}$ inear relation between drift distance and drift time.

${ }_{N}$ Final part of chamber digitization is to add cross talk. Cross talk on discriminator/preamplifier cards was simulated by computing the total amount of charge on each card, and a fraction of that charge added to all wires on the card. In the case of drift chambers, the common group is 8 wires, and 32 in proportional chamber. If a wire that gets cross talk contribution was not hit, its minimum drift distance is set to the smallest drift distance in the group in order to preserve causality.

\subsection{RICH Hits and Digitis}

Cherenkov photons in the RICH are generated by GEANT with a fixed gas density consistent with the average $\mathrm{CO}_{2}$ density during the data taking. Over the year of running, the density in the RICH varied by about $1 \%$. Given the fact that detector can be easily calibrated (see Section 7.6) one gas density for Monte Carlo is sufficient.

To generate PMT hits, photons are generated along the trajectory of the track according to the parametrized functional form of the index of refraction (see Figure 6.11). Photons are then transported to be reflected off the mirrors and then to the PMT array. Absorption in $\mathrm{CO}_{2}$, reflectivity of the mirrors, opacity of the quartz windows are taken into account in transport of photons.

Digits are generated by choosing a random number between 0 and 1 for each photon. Photons with this number above PMT efficiency for the photon's wavelength generate digits. 


\section{Chapter 9}

\section{Data Analysis}

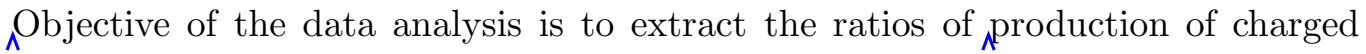
pions and kaons with momentum above $20 \mathrm{GeV} / c$ using $120 \mathrm{GeV} / \mathrm{c}_{\lambda}$ proton data on $2 \%$ interaction lengths-carbon target in bins of longitudinal and transverse particle momentum. With four particle species $\left(K^{ \pm}, \pi^{ \pm}\right)$, there are a total of six ratios, although only three are mathematically independent. In practice, the four ratios of interest are those of like charges and like species, i.e. $K^{+} / \pi^{+}, K^{-} / \pi^{-}, K^{-} / K^{+}$, and $\pi^{-} / \pi^{+}$, since systematic errors of the four ratios are different and the latter two ratios have been described phenomenologically[5] with simple relation.

\subsection{Data Binning}

\subsubsection{Geometric acceptance}

Although the objective is to sample as much of $\left(p_{z}, p_{T}\right)$ space as possible, geometric acceptance of the RICH is not perfect above $20 \mathrm{GeV} / c$. Figure 9.1 shows acceptance of the PMT array for particles with ring centers within $5 \mathrm{~cm}$ of the edge of the array. At $20 \mathrm{GeV} / c$ momentum $_{\lambda}$ kaon ring radius is approximately $15 \mathrm{~cm}, \mathrm{so}_{\Lambda}$ a significant fraction of the ring must be detectable to provide positive identification.

Given the statistics of recorded proton carbon interactions and fact that transverse momentum spectrum is steeply falling (see Figure 9.2), we limit our attention to regions of the space where geometric acceptance of the RICH is at least $50 \%$. 

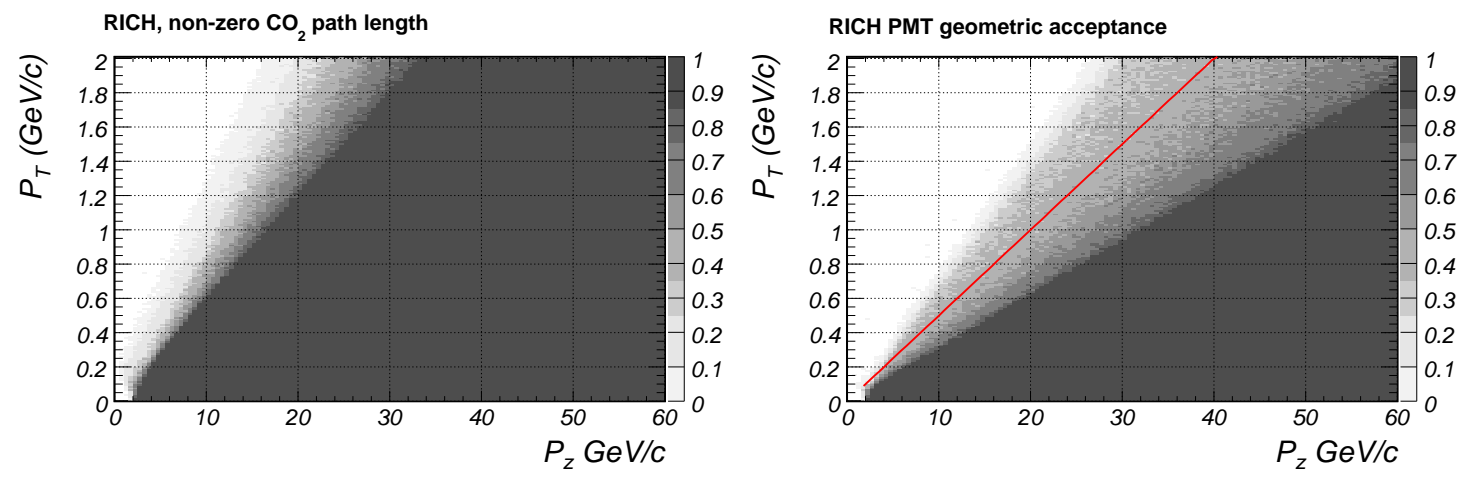

Figure 9.1: Shown are geometric acceptances for RICH radiator volume and PMT array where the ring center is within $5 \mathrm{~cm}$ of ${ }_{\Lambda} \mathrm{PMT}$ array. Line $p_{T}=0.05 p_{z}$ shows the limit for $50 \%$ geometric acceptance by the photomultipliers.
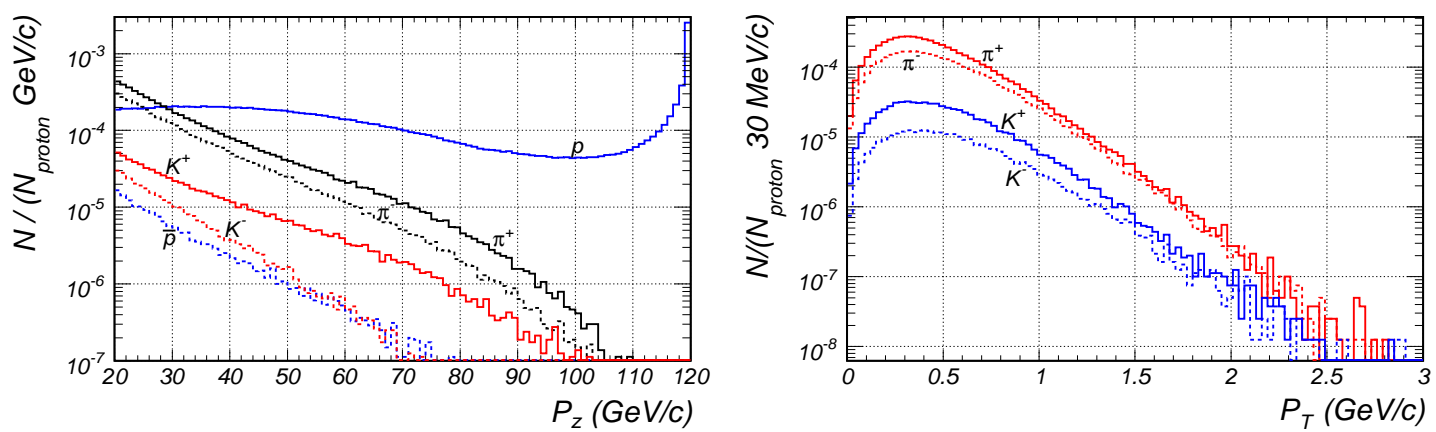

Figure 9.2: Charged hadron fluxes with longitudinal momentum above $20 \mathrm{GeV} / c$ per incident proton as simulated by FLUKA-06 on $2 \%$ carbon target. 


\subsubsection{Bin Selection}

The choice of upper and lower limits on $p_{z}$ is governed by two considerations. $\Lambda^{K a o n}$ threshold is around $17 \mathrm{GeV} / c$, thus $20 \mathrm{GeV} / c$ momentum $_{\Lambda}$ with $\sim 5 \%$ momentum resolution ensures minimal bias from the threshold effect $\hat{\Lambda}_{\AA}$ which we are unable to measure from the data at this time. Particle production with momentum $\mathrm{A}_{\lambda}$ above $90 \mathrm{GeV} / c$ is heavily dominated by inelastically scattered protons, so cutting the right bin at $p_{z}<90 \mathrm{GeV} / c$ will reduce the background underneath $K^{+} K^{+}$peak and remove a small number of true kaons and pions.

In the limit of infinite statistics, one would like to have the smallest bins possible. However with $5 \cdot 10^{5}$ recorded triggers and estimated $50 \%$ interaction trigger purity, we must select sufficiently large bins in order to have a statistically significant measurement. Figure 9.3 shows bin by bin the expected number of $\pi^{+}$from $2 \cdot 10^{5}$ proton-carbon interactions modeled in FLUKA-06. Of the four particles of interest, $\pi^{+}$is the majority particle, hence at the very least one needs to have a significant number of pions to do the measurement. The criteria used to select bin boundaries was to get $3 \%$ or better statistical error on $\pi^{+}$if possible. On the other hand, momentum resolution is at most $5.5 \%$, so using that as the upper limit, we select at least $20 \%$ bins so that mixing between bins is minimized. The 24 bins defined for the measurement are shown in Table 9.1.

\subsection{Pileup Removal}

Resonant extraction from the Main Injector could not be tuned to guarantee a single particle per $10 \mu$ s cycle in the machine, therefore a significant fraction of triggers contain two or more incident protons. While the probability of both protons to interact is small, pileup can create confusion in vertex finding and fitting. Since this bias is not modeled in the Monte Carlo (MC), it is best to remove pileup events from the analysis.

In order not to bias the measurement, pileup removal has to be done using information from detectors upstream of the target as much as possible. This includes

- Number of tracks in the beam chambers, and beam track time;

- Three scintillator counters (charge and hit time differences);

- Four beam Cherenkov PMT signals (charge and hit times); 


\begin{tabular}{c|rr|rr}
\hline \hline Bin & Minimum $p_{z}$ & Minimum $p_{z}$ & Maximum $p_{T}$ & Maximum $p_{T}$ \\
\hline$(0,0)$ & 20 & 24 & 0 & 0.2 \\
$(0,1)$ & 20 & 24 & 0.2 & 0.4 \\
$(0,2)$ & 20 & 24 & 0.4 & 0.6 \\
$(0,3)$ & 20 & 24 & 0.6 & 1.0 \\
\hline$(1,0)$ & 24 & 31 & 0 & 0.2 \\
$(1,1)$ & 24 & 31 & 0.2 & 0.4 \\
$(1,2)$ & 24 & 31 & 0.4 & 0.6 \\
$(1,3)$ & 24 & 31 & 0.6 & 1.0 \\
$(1,4)$ & 24 & 31 & 1.0 & 1.2 \\
\hline$(2,0)$ & 31 & 42 & 0 & 0.2 \\
$(2,1)$ & 31 & 42 & 0.2 & 0.4 \\
$(2,2)$ & 31 & 42 & 0.4 & 0.6 \\
$(2,3)$ & 31 & 42 & 0.6 & 1.0 \\
$(2,4)$ & 31 & 42 & 1.0 & 1.55 \\
\hline$(3,0)$ & 42 & 60 & 0 & 0.2 \\
$(3,1)$ & 42 & 60 & 0.2 & 0.4 \\
$(3,2)$ & 42 & 60 & 0.4 & 0.6 \\
$(3,3)$ & 42 & 60 & 0.6 & 1.0 \\
$(3,4)$ & 42 & 60 & 1.0 & 2.0 \\
\hline$(4,0)$ & 60 & 90 & 0 & 0.2 \\
$(4,1)$ & 60 & 90 & 0.2 & 0.4 \\
$(4,2)$ & 60 & 90 & 0.4 & 0.6 \\
$(4,3)$ & 60 & 90 & 0.6 & 1.0 \\
$(4,4)$ & 60 & 90 & 1.0 & 2.0 \\
\hline \hline
\end{tabular}

Table 9.1: $\left(p_{z}, p_{T}\right)$ bins selected for ratio measurement. 


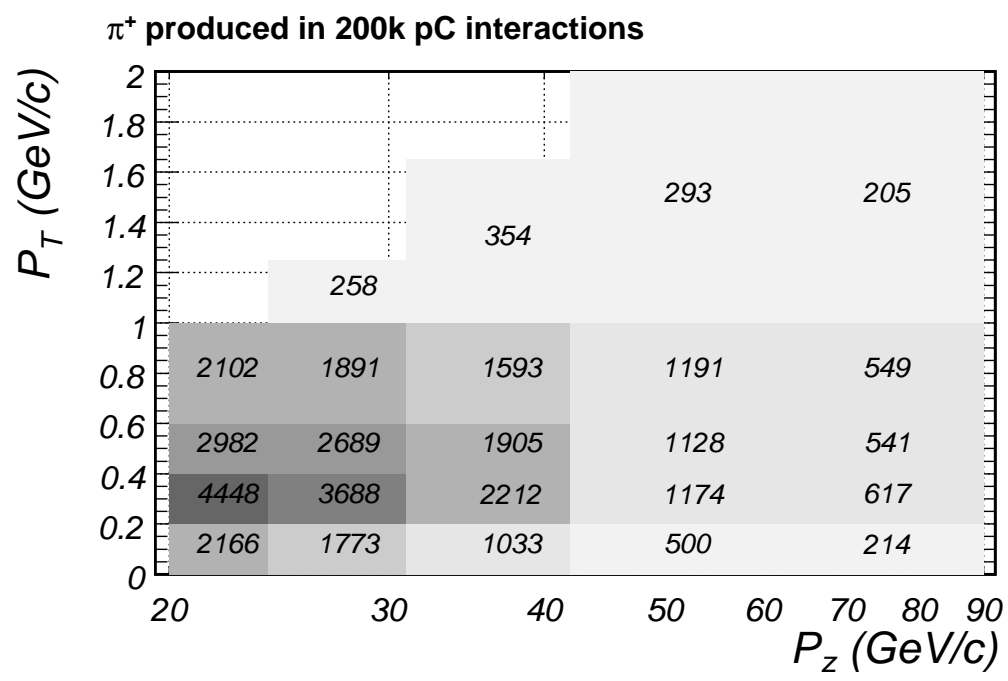

Figure 9.3: Expected number of $\pi^{+}$particles in the bins selected for the ratio measurement. Bin boundaries in $p_{z}$ are $20,24,31,42,60,90 \mathrm{GeV} / c$, and in $p_{T} 0.2,0.4,0.6,1 \mathrm{GeV} / c$. The largest $p_{T}$ bin stretches to $2 \mathrm{GeV} / c$ or 0.05 of the lower bin boundary, which corresponds to $50 \%$ geometric acceptance.

- Times of reconstructed secondary tracks.

We can easily $\mathbf{y}_{\Lambda}$ select pileup events by looking at the amount of energy deposited by uninteracted proton tracks inside the TPC. Figure 9.4 shows that when two or more tracks are on top of one another, ${ }_{\Lambda}$ TPC integrates the total ionization energy resulting in multiple peaks in $d E / d x$. One has to keep in mind that the chamber integrates over $15 \mu \mathrm{s}$, while most detectors keep information in a $100 \mathrm{~ns}$ window, and chamber TDCs have $1 \mu \mathrm{s}$ window. Therefore a fraction of events which look like pileup in the TPC will create normal response in all other detectors.

Events with $d E / d x<3$ were taken to come from clean events, whereas those with

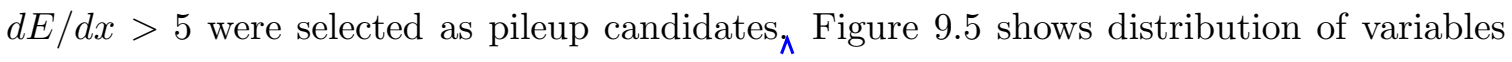
which are most helpful to select between clean and pileup events ${ }_{\mathcal{K}}$ In order of significance, these $\operatorname{are}_{\lambda}$

1. Number of beam tracks is greater than 1;

2. Average charge of beamline scintillator counters is greater than 1.8;

3. ${ }_{\wedge}$ Beam track time is outside of -5 to $20 \mathrm{~ns}$ window; 

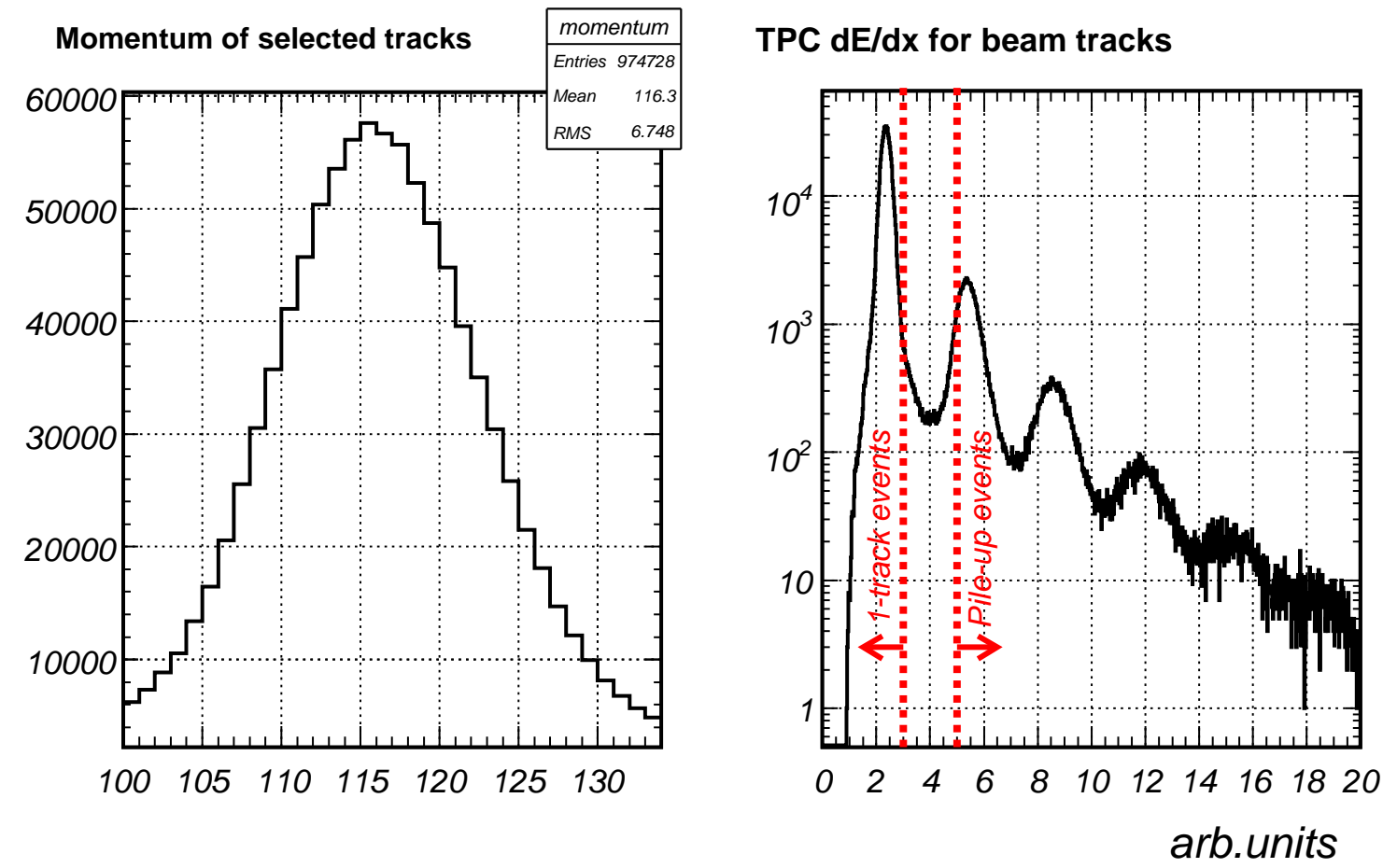

Figure 9.4: Momentum (in $\mathrm{GeV} / c$ ) and energy loss of beam tracks in the TPC where single and pileup proton tracks are easily identified. 
4. Large (greater than $1 \mathrm{ns)} \mathrm{time} \mathrm{difference} \mathrm{of} \mathrm{signals} \mathrm{in} \mathrm{T0} \mathrm{counters;}$

5. Average charge of beam Cherenkov charge is greater than 330 ADC counts;

6. Inconsistent TDC values of beam Cherenkov counters,

One additional cut is placed on the time of secondary tracks. This is the only cut that does involve data from detectors downstream of the target. However, as Figure 9.6 shows, in case of single-proton tracks selected for pileup study, only $1 \%$ of events with no pileup candidates have out of time secondary tracks and $45 \%$ of pileup candidates have out of time secondary tracks. Thus, requiring that all tracks come in between -15 and $55 \mathrm{~ns}$ removes additional sources of pileup.

In addition to $d E / d x$ in the TPC, additional variable that helps us judge the performance of pileup removal is the amount of energy deposited in the hadron calorimeter. Figure 9.7 shows that the cuts clean up energy deposition in the TPC volume and in the hadron calorimeter. While about $20-40 \%$ of the data contains pileup after these simple cuts less than $1 \%$ of the data contains events with pileup.

\subsection{Primary Vertex Selection}

With $6 \mathrm{~mm}$ vertex resolution in $z$, the cuts on target-like events needs to be done in such a way so as not to introduce a bias into the measurement. We open a wide window to include both the target and the scintillator, and subtract appropriately scaled target ${ }_{\AA}$ out data.

To determine the fraction of target out data that needs to be subtracted from target $\lambda_{\lambda}$ in data, we plot distributions of vertex $z$ in each set in pileup free events. The shape of the $z$ distribution is well described by a Gaussian with exponential tails on both sides plus a constant background. First, we make a fit to the target out data to determine the shape of the trigger counter peak. To fit for the target in distribution, we hold all parameters of the scintillator peak fixed except for the amplitude. Figure 9.8 shows that this technique does a good job at fitting for the carbon data $z$ distribution and tells us that the available data with target out needs to be scaled by $0.934 \pm 0.026$ before it is subtracted from the $2 \%$ carbon data to account for the presence of the scintillator behind the target ${ }_{\lambda}$ Based on this plot we can tell that by defining target region from -832 to $-825 \mathrm{~cm}$ in $z$ will eliminate any potential bias due to cuts. 

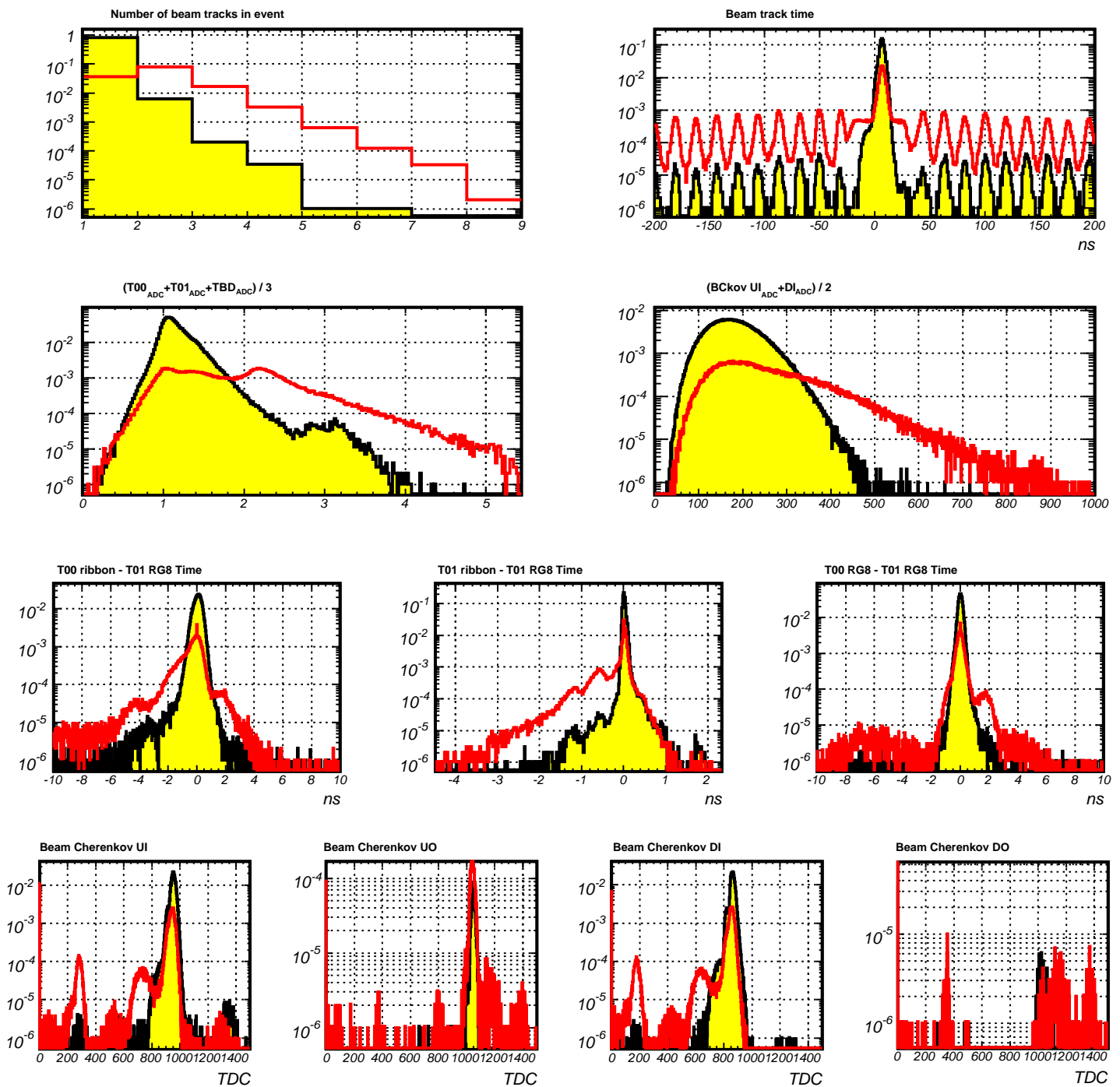

Figure 9.5: Distribution of variables that help distinguish pileup events from clean events. Solid yellow histograms are filled with information from single-track candidates, and red histograms from pileup candidate events. The histograms are scaled by the total number $\left(5.7 \cdot 10^{5}\right)$ of proton tracks selected for the pileup study. 

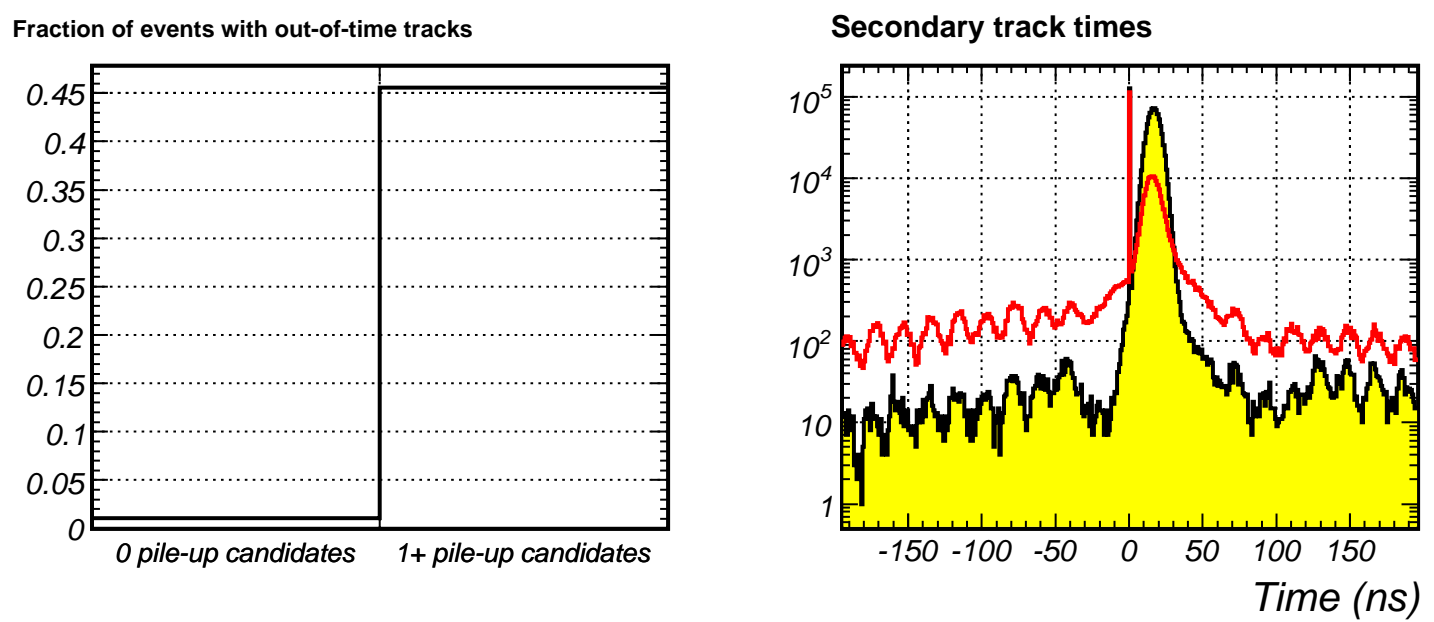

Figure 9.6: On the left is fraction of events containing out of time secondary tracks in single proton candidate and pileup candidate events. On the right is distribution of times for the two classes of events. The peak at 0 ns corresponds to tracks that have no information from the drift chambers.
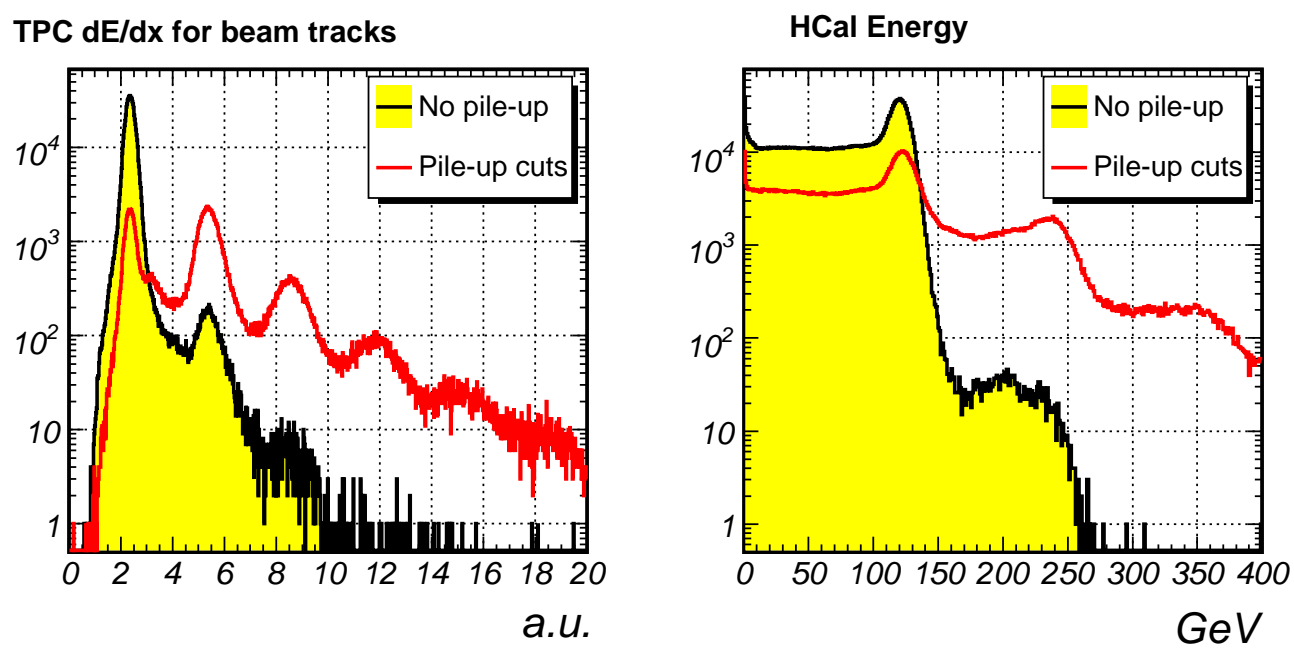

Figure 9.7: pileup cuts are good at removing multiple peaks from TPC $d E / d x$ of beam tracks and very high calorimeter energy. Less that $1.5 \%$ of beam tracks have measured $d E / d x$ consistent with two or more particles and less than $0.5 \%$ of events deposit more energy than a single $120 \mathrm{GeV} / c$ proton. 


\section{Primary vertex $\mathbf{Z}$}

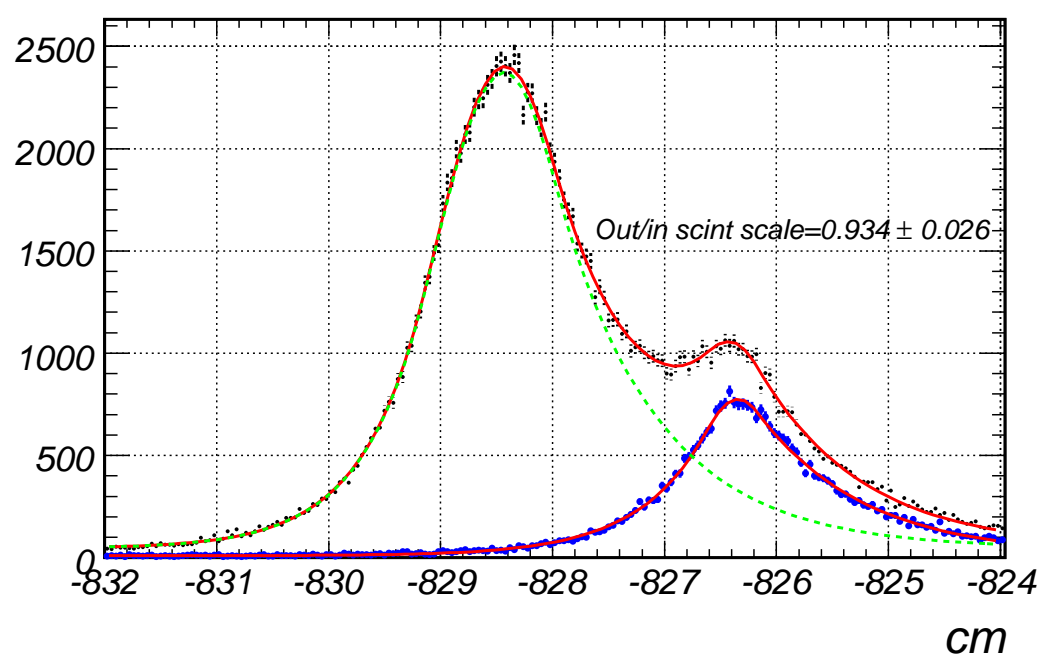

Figure 9.8: 2\% carbon and target out data. The scale factor of available thin target data is based on fitting $\Lambda_{\Lambda}$ Gaussian with exponential tails to the target and the interaction counter.

\begin{tabular}{|l|rrrrrrrr|}
\hline & \multicolumn{8}{|c|}{ Vertex multiplicity } \\
& 2 & 3 & 4 & 5 & 6 & 7 & 8 & 9 \\
\hline Highest efficiency & 0.212 & 0.852 & 0.903 & 1.000 & 1.000 & 1.000 & 1.000 & 1.000 \\
Lowest efficiency & 0.107 & 0.650 & 0.826 & 0.923 & 0.818 & 0.837 & 0.916 & 0.952 \\
Average efficiency & 0.158 & 0.751 & 0.863 & 0.962 & 0.909 & 0.919 & 0.958 & 0.976 \\
\hline
\end{tabular}

Table 9.2: Trigger efficiency using beryllium and carbon data.

\subsection{Interaction Trigger Efficiency}

Interaction trigger efficiency needs to be understood as multiplicities of events containing kaons and pions may be somewhat different. To remove bias from calculation of the efficiency, we selected interactions from events where minimum bias trigger recorded the event. For the study of interaction trigger efficiency, we selected events with no pileup and vertex $z$ between -831 and $-827 \mathrm{~cm}$.

Trigger efficiency does not only depend on the properties of the scintillator, but also on event topology. Therefore, it is not surprising that measured efficiency using beryllium or carbon data is different from efficiency computed using bismuth data. To quantify effect of the trigger efficiency, we rely upon the beryllium and carbon minimum bias events to get the lowest and the highest efficiency in each multiplicity bin (see Table 9.2) 


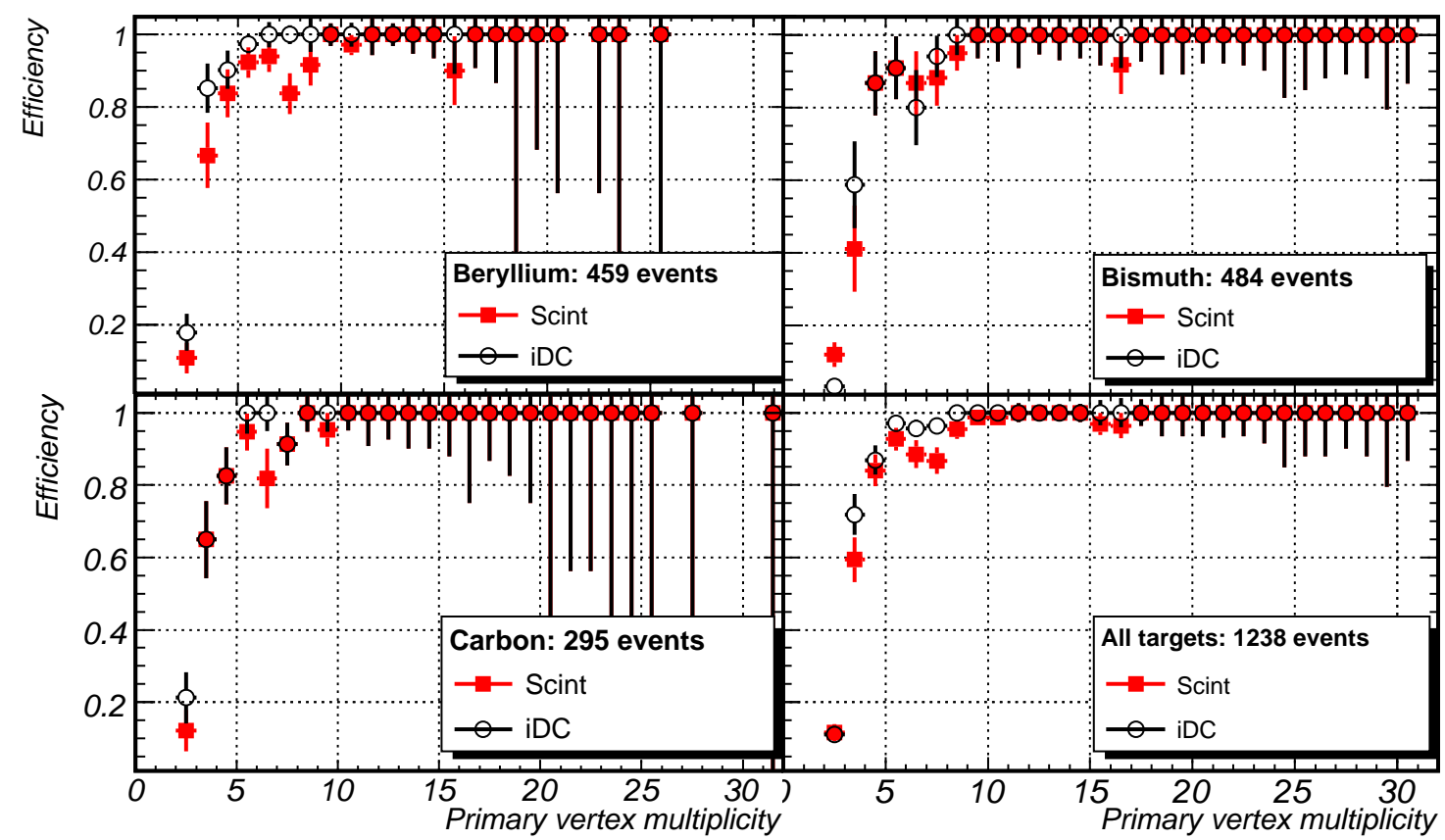

Figure 9.9: Interaction trigger efficiency for different targets at $120 \mathrm{GeV} / c$ and the combined trigger efficiency for all targets.

\subsection{Particle ID Variable}

Computed mass squared using ${ }_{\Lambda} \mathrm{RICH}$ ring radius varies with particle $p_{z}$ and $p_{T}$ making it impossible to directly compare data and Monte Carlo simulation (see Figures 9.11 and 9.12) $)_{\kappa}$ To alleviate this problem, we create a particle ID variable $\alpha$ through

$$
\alpha\left(m^{2}\right)=a_{i j}+b_{i j} m^{2}
$$

where $a_{i j}$ and $b_{i j}$ are different from bin to bin. We defined $\alpha=0$ to correspond to pion and $\alpha=1$ to proton. Then kaon peak will be found at 0.265 .

To find $a_{i j}$ and $b_{i j}$, in each $\left(p_{z}, p_{t}\right)$ bin we group negatives and positives from carbon, beryllium, and target out data together. Distributions of $m^{2}$ are fit for pion and proton peak or kaon if proton is under threshold in both data and Monte Carlo. Peak positions are then converted into coefficients through

$$
\begin{aligned}
& b=\left[m_{p}^{2}-m_{\pi}^{2}\right]^{-1} \\
& a=-b m_{\pi}^{2}
\end{aligned}
$$




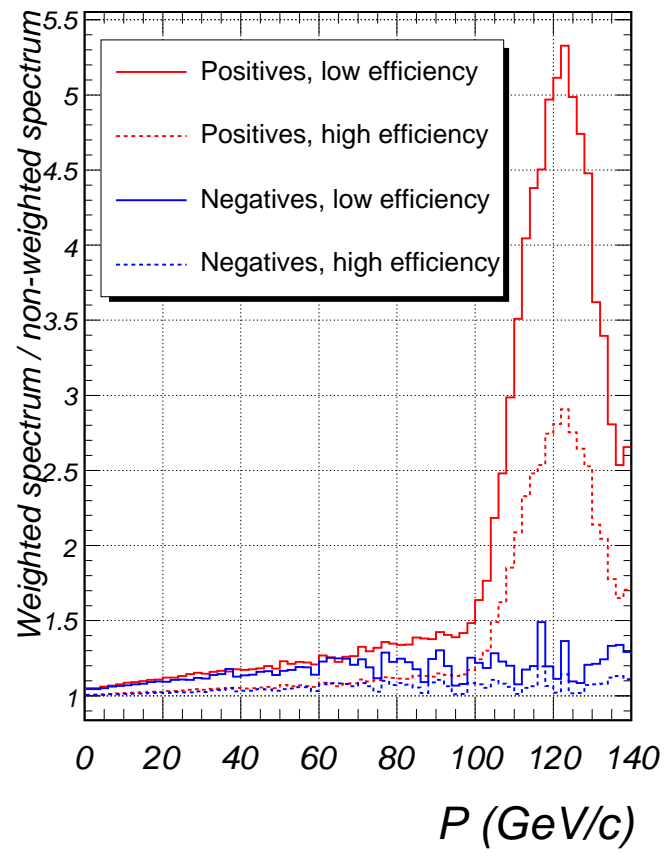

Figure 9.10: Combined carbon, beryllium, and target out momentum spectrum enhancement under the assumptions of the most efficient and the most inefficient trigger. 


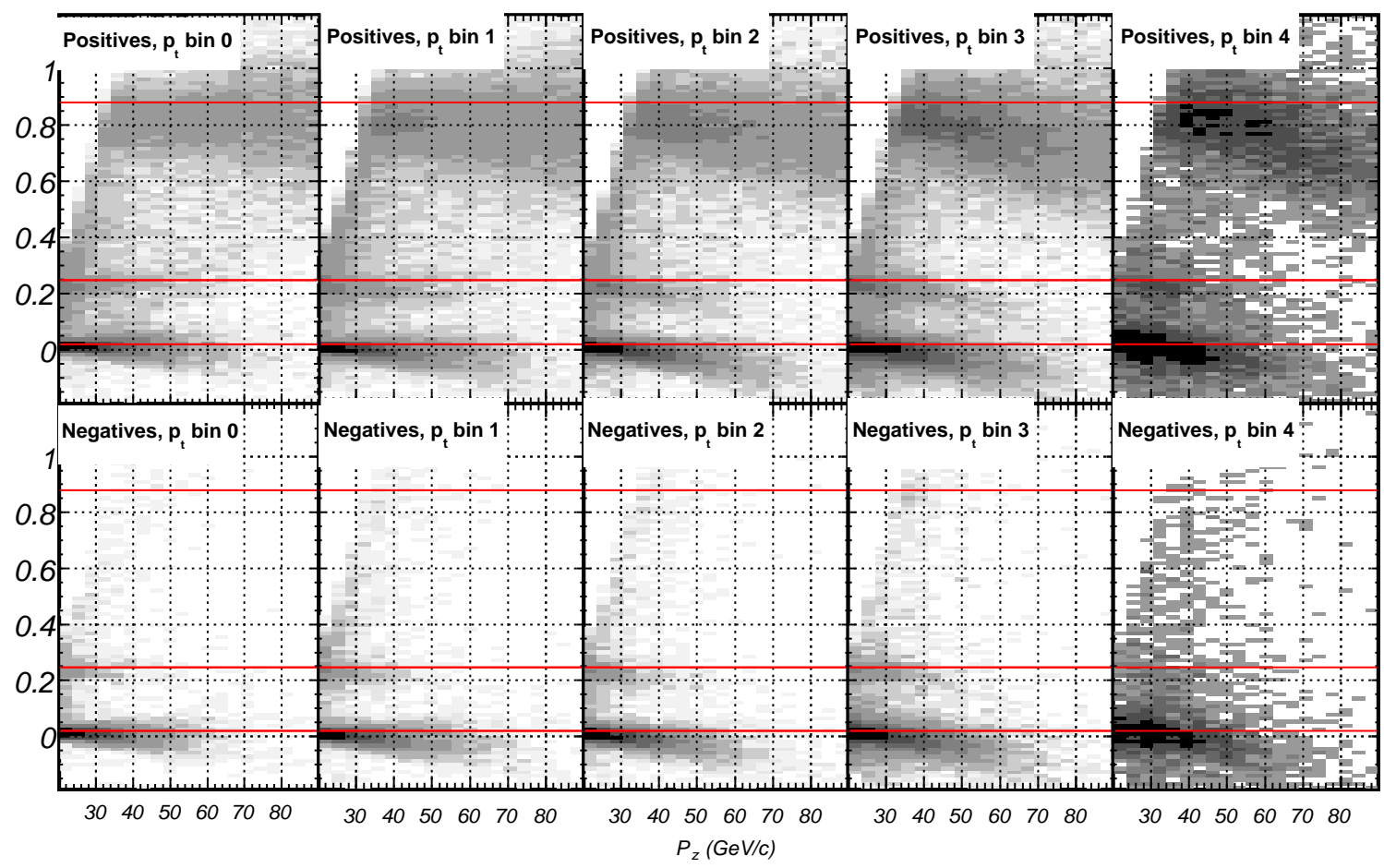

Figure 9.11: Variations of computed mass squared with $p_{z}$ and $p_{t}$ make it impossible to directly compare data and Monte Carlo. Superimposed are horizontal lines corresponding to mass squared of pions, kaons, and protons. 


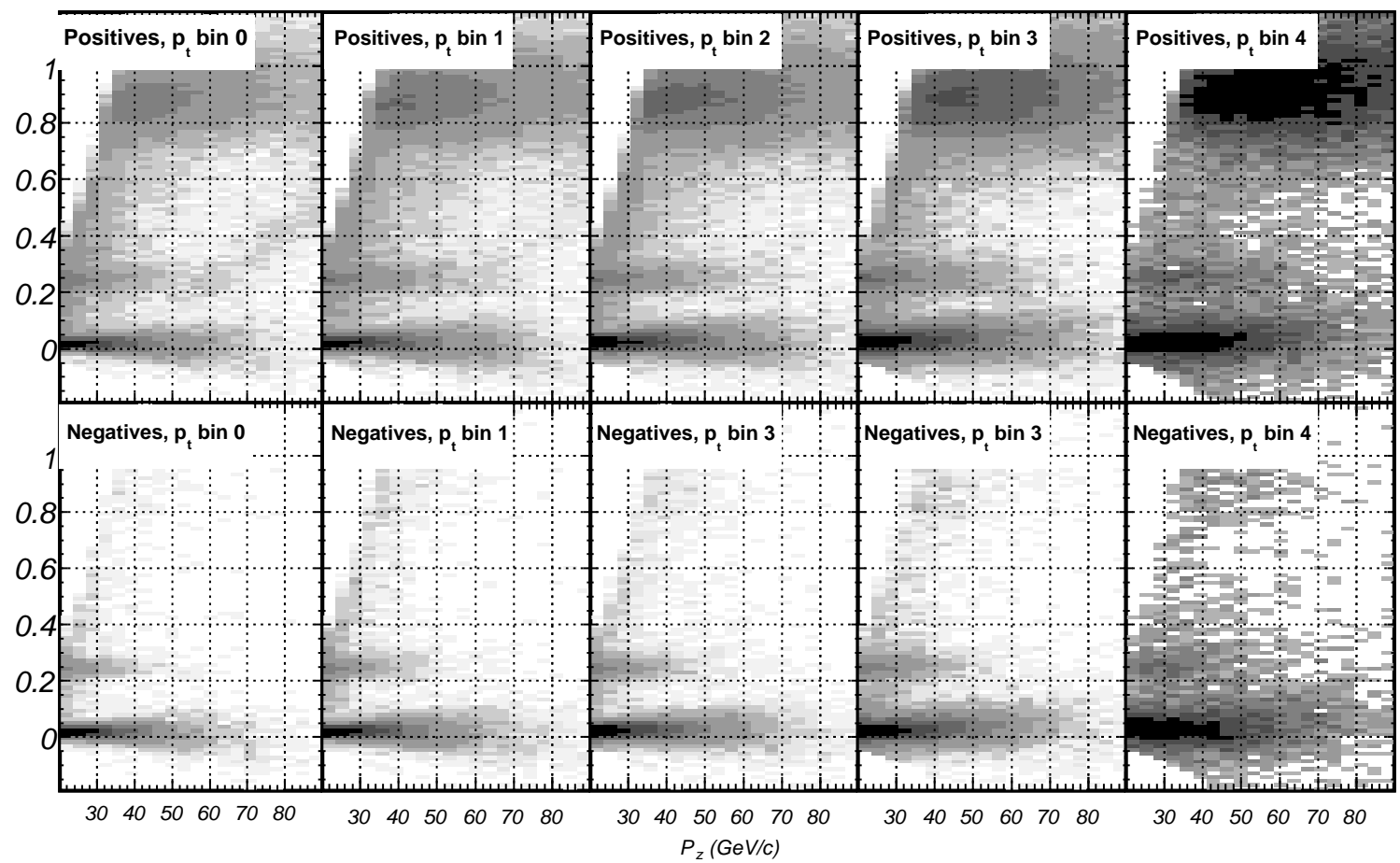

Figure 9.12: Variations of computed mass squared observed in data are not present in Monte Carlo. 


\begin{tabular}{|cc|rrrr|rr|}
\hline \hline & & \multicolumn{6}{|c|}{ Data } \\
$p_{z}$ bin & $p_{t}$ bin & $m_{\pi}^{2} \cdot 10^{3}$ & $m_{p}^{2} \cdot 10^{3}$ & $a_{i j} \cdot 10^{3}$ & $b_{i j}$ & $a_{i j} \cdot 10^{3}$ & $b_{i j}$ \\
\hline 0 & 0 & $12.9 \pm 0.1$ & $228.4 \pm 1.2$ & -15.9 & 1.230 & -23.8 & 1.171 \\
0 & 1 & $8.4 \pm 0.1$ & $229.3 \pm 0.9$ & -10.1 & 1.199 & -25.3 & 1.175 \\
0 & 2 & $8.5 \pm 0.1$ & $229.8 \pm 1.1$ & -10.1 & 1.197 & -25.7 & 1.170 \\
0 & 3 & $12.2 \pm 0.2$ & $228.2 \pm 1.2$ & -14.9 & 1.226 & -26.1 & 1.172 \\
\hline 1 & 0 & $11.0 \pm 0.2$ & $228.4 \pm 1.2$ & -13.4 & 1.219 & -23.1 & 1.161 \\
1 & 1 & $4.0 \pm 0.1$ & $222.8 \pm 0.9$ & -4.8 & 1.211 & -26.2 & 1.175 \\
1 & 2 & $2.3 \pm 0.1$ & $223.3 \pm 0.7$ & -2.8 & 1.199 & -27.5 & 1.172 \\
1 & 3 & $4.2 \pm 0.2$ & $225.5 \pm 1.1$ & -5.1 & 1.198 & -27.2 & 1.169 \\
1 & 4 & $13.3 \pm 1.4$ & $219.2 \pm 3.4$ & -17.2 & 1.287 & -29.0 & 1.168 \\
\hline 2 & 0 & $8.2 \pm 0.3$ & $794.5 \pm 2.2$ & -10.5 & 1.272 & -22.3 & 1.208 \\
2 & 1 & $-3.6 \pm 0.2$ & $792.4 \pm 1.3$ & 4.6 & 1.256 & -27.1 & 1.211 \\
2 & 2 & $-8.9 \pm 0.2$ & $799.4 \pm 1.2$ & 11.1 & 1.237 & -32.4 & 1.202 \\
2 & 3 & $-9.2 \pm 0.3$ & $804.7 \pm 1.3$ & 11.3 & 1.229 & -32.4 & 1.196 \\
2 & 4 & $-1.9 \pm 1.3$ & $803.3 \pm 3.9$ & 2.4 & 1.242 & -32.7 & 1.188 \\
\hline 3 & 0 & $2.0 \pm 0.8$ & $810.0 \pm 1.7$ & -2.5 & 1.238 & -15.8 & 1.147 \\
3 & 1 & $-15.3 \pm 0.6$ & $807.6 \pm 1.0$ & 18.5 & 1.215 & -22.0 & 1.150 \\
3 & 2 & $-27.2 \pm 0.6$ & $797.4 \pm 1.0$ & 32.9 & 1.213 & -31.9 & 1.160 \\
3 & 3 & $-33.1 \pm 0.6$ & $791.0 \pm 1.0$ & 40.2 & 1.214 & -40.1 & 1.164 \\
3 & 4 & $-27.6 \pm 1.9$ & $810.1 \pm 2.8$ & 33.0 & 1.194 & -38.9 & 1.151 \\
\hline 4 & 0 & $-11.9 \pm 2.8$ & $813.6 \pm 1.7$ & 14.4 & 1.211 & -8.6 & 1.111 \\
4 & 1 & $-34.7 \pm 2.0$ & $793.6 \pm 1.2$ & 41.9 & 1.207 & -11.5 & 1.116 \\
4 & 2 & $-58.2 \pm 2.2$ & $766.3 \pm 1.3$ & 70.6 & 1.213 & -24.8 & 1.132 \\
4 & 3 & $-67.5 \pm 2.2$ & $744.2 \pm 1.4$ & 83.2 & 1.232 & -48.7 & 1.150 \\
4 & 4 & $-80.9 \pm 5.7$ & $726.7 \pm 3.5$ & 100.1 & 1.238 & -61.2 & 1.150 \\
\hline \hline
\end{tabular}

Table 9.3: Coefficients for conversion from $m^{2}$ in $\left(\mathrm{GeV} / c^{2}\right)^{2}$ into particle ID variable $\alpha$. For $p_{z}$ bins 0 and 1 , the numbers were computed using pion and kaon peak since proton is under threshold below $31 \mathrm{GeV} / c$. Ideal $a_{i j}=-22.45 \cdot 10^{-3}$ and $b_{i j}=1.162\left(\mathrm{GeV} / c^{2}\right)^{2}$. 


\subsection{Corrections from Monte Carlo Simulation}

\subsubsection{Need for Corrections}

A number of effects which one cannot easily extract from data must be corrected for. These include

1. Interactions in the spectrometer: about $10 \%$ of secondary particles will interact before reaching the $\mathrm{RICH}$;

2. Interactions in the RICH flange: about $10 \%$ of particles passing through the flange rather than the RICH window will interact;

3. Decay in flight: about $10 \%$ of kaons will decay before they reach the RICH;

4. Particle misidentification due to RICH efficiency and ring fitting efficiency;

5. Particle misidentification due to multiple scattering: large angle scattering of a small fraction of protons (majority particle above $\sim 30 \mathrm{GeV} / c$ ) will create a measurable contamination, especially under the kaon peak;

6. Decay products of neutral particles (e.g. $K_{S}, \Lambda$ ) that get incorrectly pulled into the primary vertex.

7. Interactions in the trigger counter;

Figures 9.13 and 9.14 show that data and Monte Carlo distributions of the particle ID variable compare well in most bins, although Monte Carlo peaks are somewhat narrower.

\subsubsection{Background Modeling}

In order to extract correct numbers of pions and kaons, one has to evaluate the shape of the background in the particle ID variable and subtract the background from underneath the peaks. Figure 9.15 shows distribution of reconstructed Monte Carlo mass squared in one of the bins. As one would expect, protons are the primary source of background under the kaon peak in the bin where proton is under threshold. What is more surprising that a significant number of true protons get identified as pions. The possible explanations for this are 


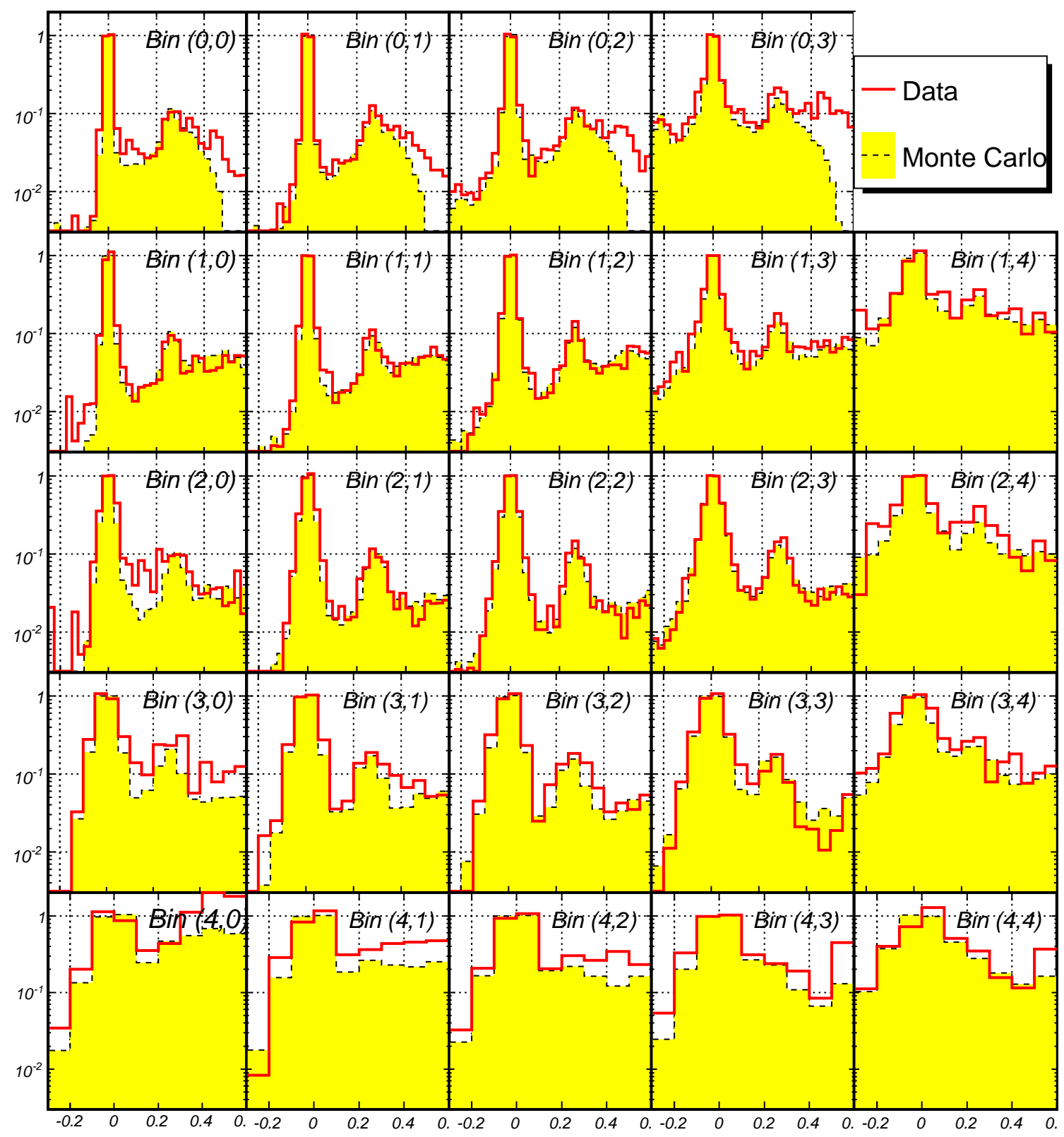

Figure 9.13: Data - Monte Carlo comparison of particle ID variable distributions for positives in the $\left(p_{z}, p_{t}\right)$ bins. Histograms are normalized to set the pion peak to 1 . 


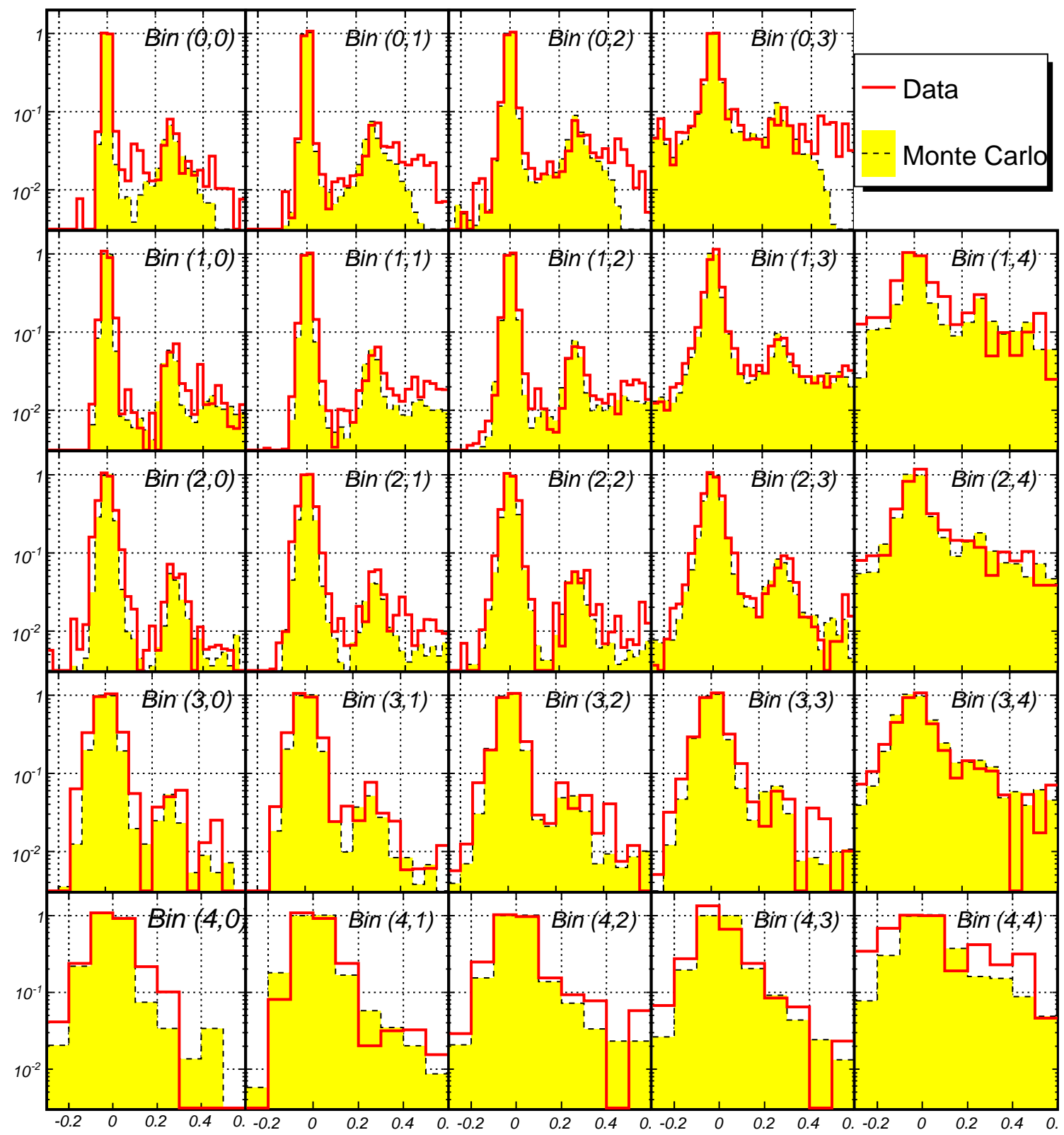

Figure 9.14: Data - Monte Carlo comparison of particle ID variable distributions for negatives in the $\left(p_{z}, p_{t}\right)$ bins. Histograms are normalized to set the pion peak to 1 . 
MC Positives, bin $(0,0)$

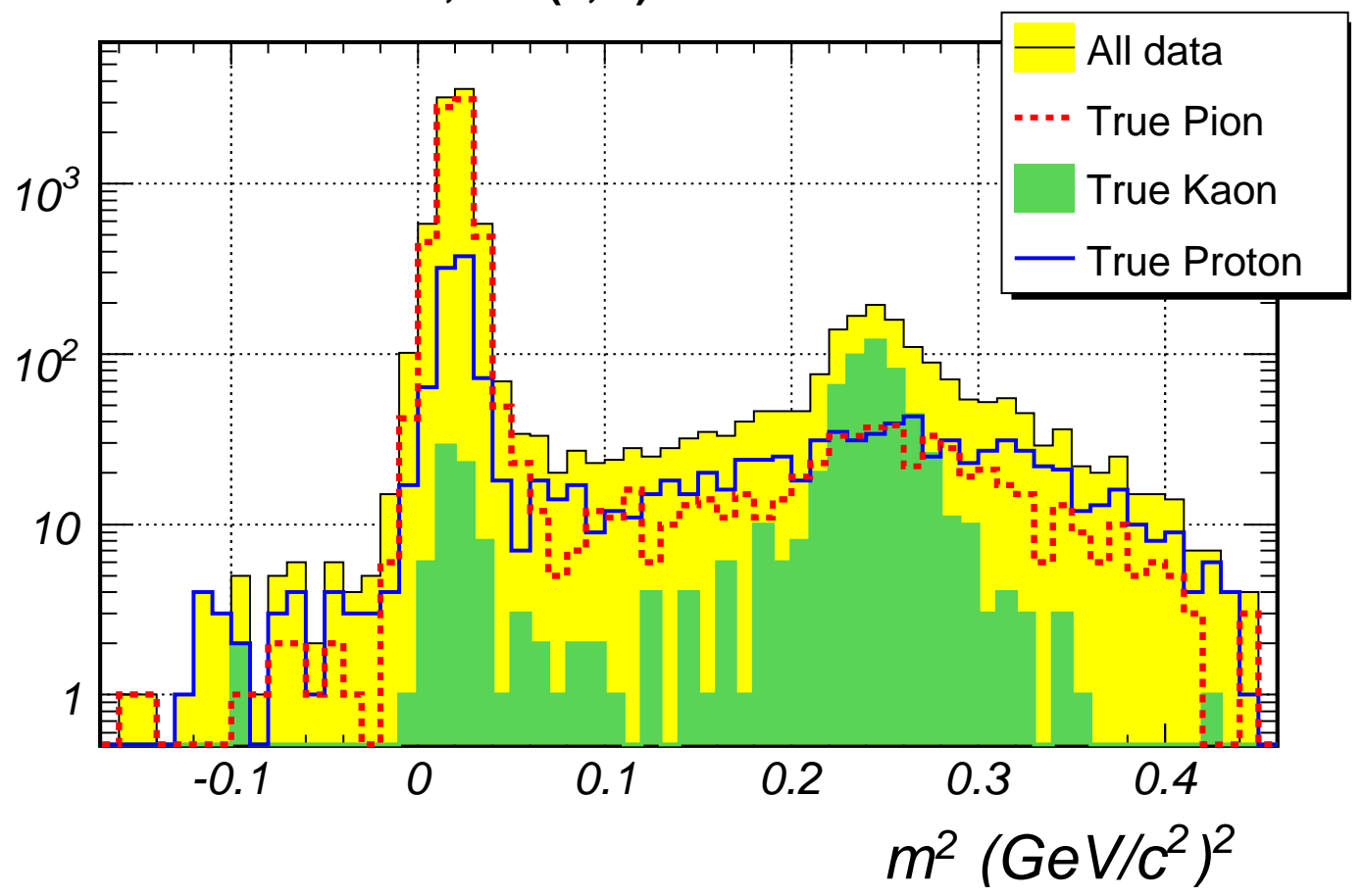

Figure 9.15: Reconstructed Monte Carlo mass squared in the first bin. It is clear that background has to be subtracted in order to extract correct number of kaons.

- Protons interacting in the spectrometer that create a forward pion or electron so that the track is extended into the RICH and get identified as pions;

- Incorrect reconstructed - true track association.

Data - Monte Carlo comparison is the best model of background that we have, so to extract the signal from the background, we use true Monte Carlo information to extract the occupancy o of pions and kaons in the bins of particle ID histograms. Pion (kaon) occupancy is defined as the fraction of true pions (kaons) with reconstructed momentum within $4 \sigma$ of true momentum. The highest occupancy (see Figures 9.16 and 9.17) almost never goes above 0.8. The order of magnitude of the maximum pion occupancy is consistent with our expectation that $\sim 14 \%$ of protons will interact in the spectrometer.

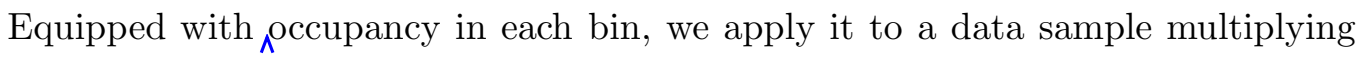
every bin of the data binned the same way by the computed occupancy. Then the number of pions in kaons is simply the integral of the derived histograms. The error on the count of particles is the sum of errors in each bin added in quadrature. 


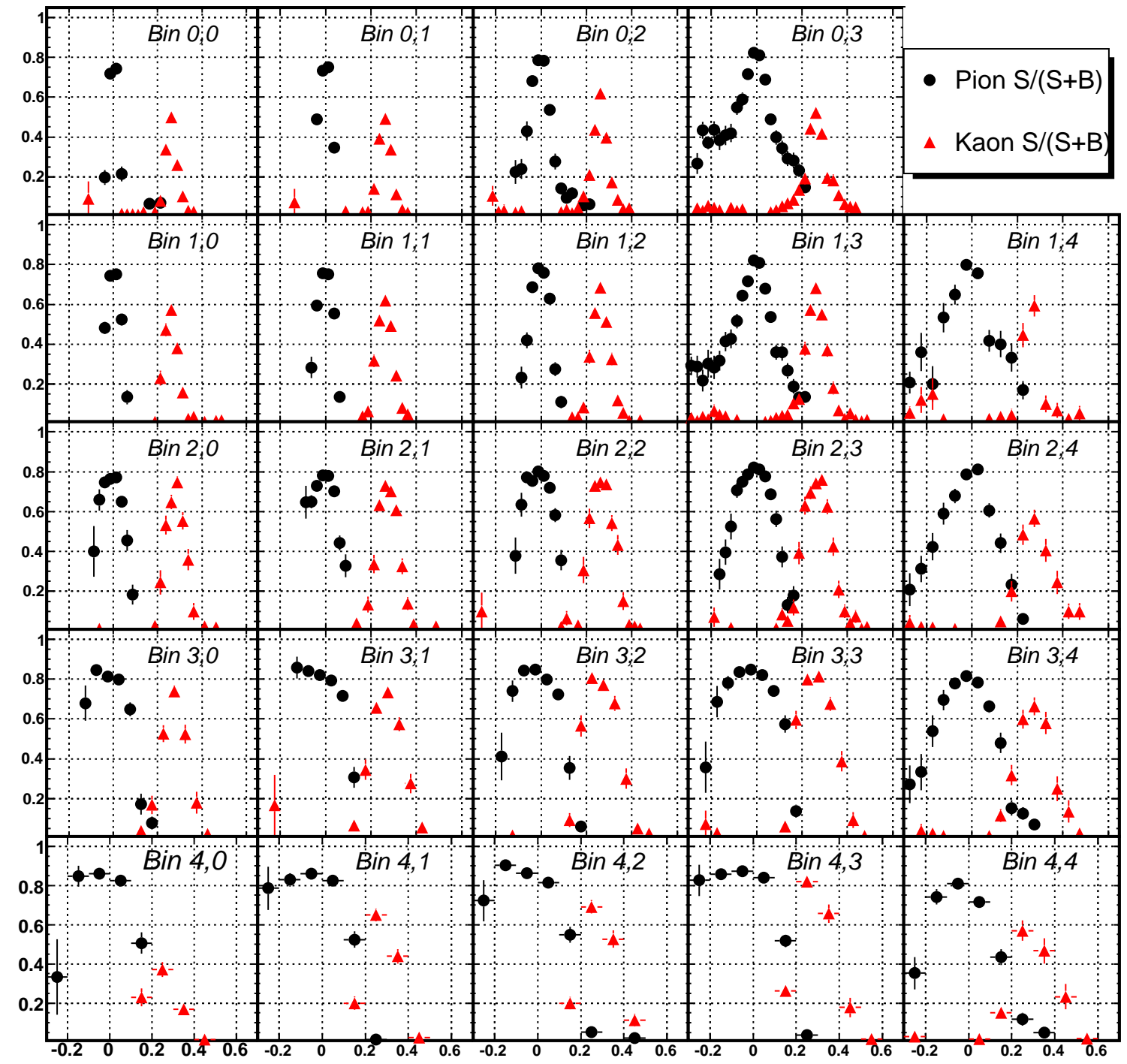

Figure 9.16: Occupancy of positive pions and kaons in Monte Carlo in the $\left(p_{z}, p_{t}\right)$ bins. $\wedge$ 


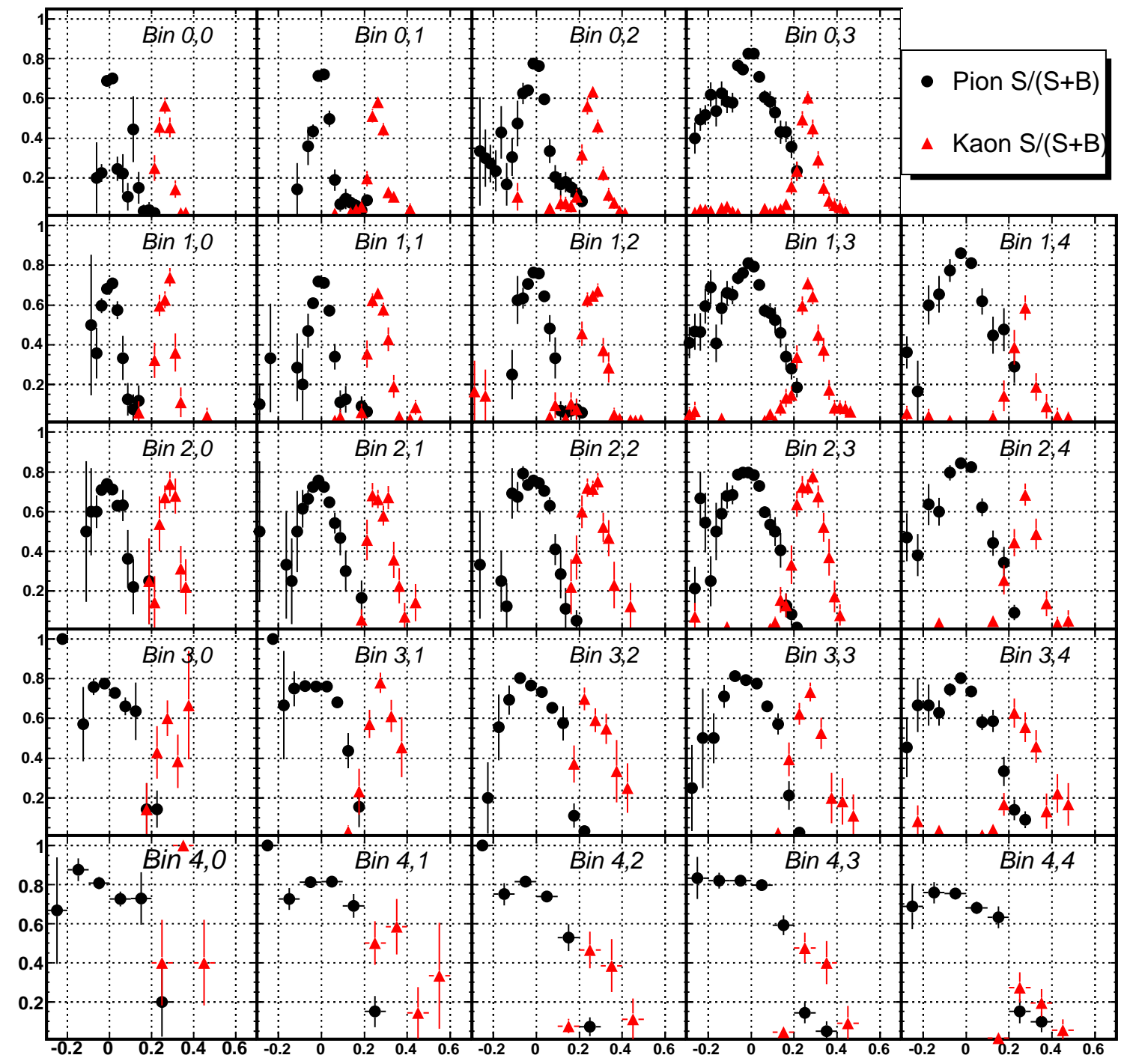

Figure 9.17: Occupancy of negative pions and kaons in Monte Carlo in the $\left(p_{z}, p_{t}\right)$ bins. 

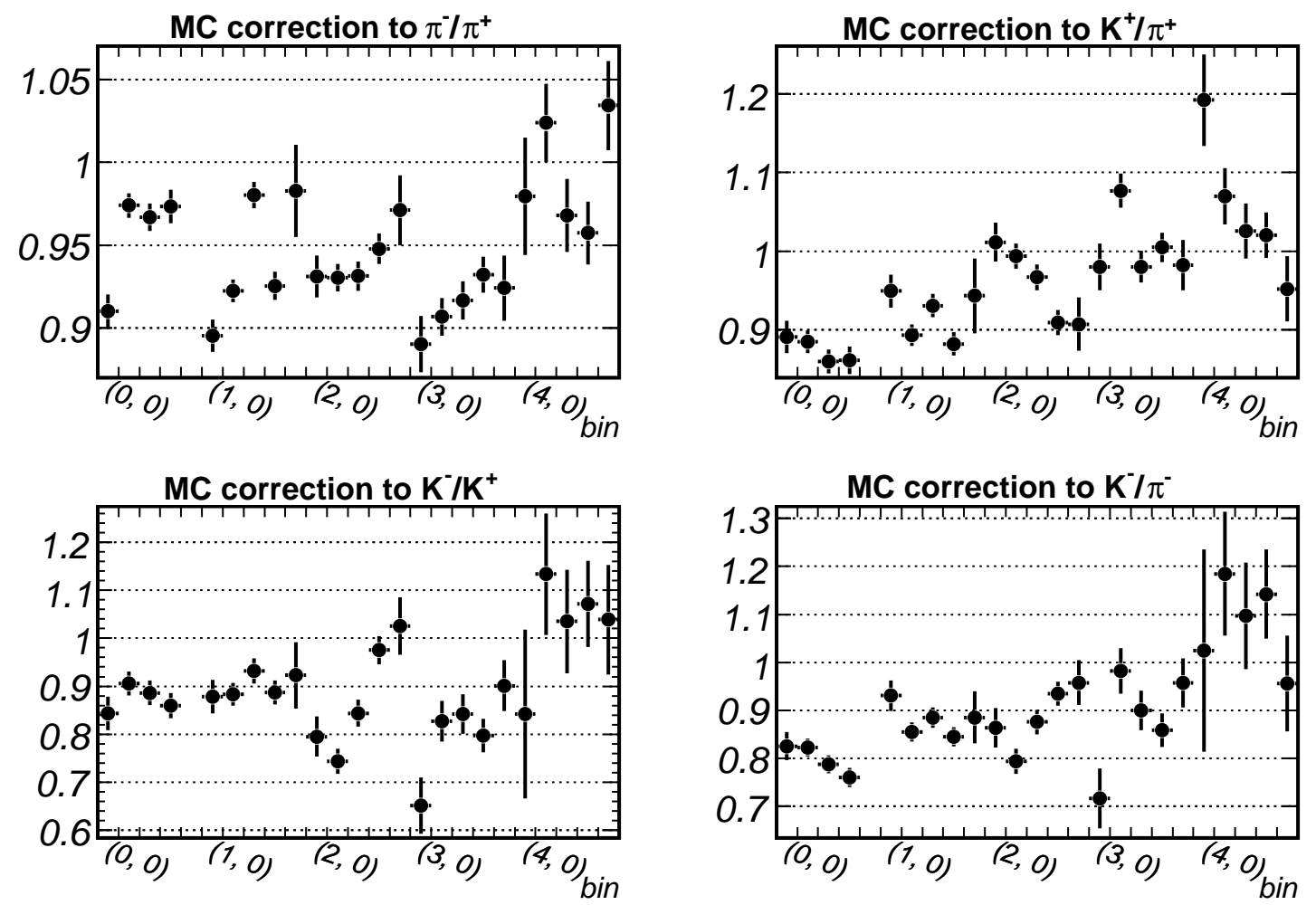

Figure 9.18: Ratios of reconstructed Monte Carlo ratios and true Monte Carlo ratios. The error bars come from propagation of statistical errors on the ratios, which are correlated between the reconstructed and true ratios.

\subsection{Corrections and Errors}

We can judge how well the analysis method performs by comparing ratios derived from reconstructed Monte Carlo to true ratios in each $\left(p_{z}, p_{T}\right)$ bin. Results shown in Figure 9.18 indicate that typical corrections are less than 10\%. Given our current modeling of detectors in the Monte Carlo simulation, we will assign a conservative $50 \%$ systematic uncertainty on the applied correction.

As expected, errors associated with imperfect knowledge of interaction trigger efficiency are quite small with the exception of the bins which have little statistics. To compute the error associated with trigger efficiency, we compute the ratios using the upper estimate of efficiency, giving each identified particle the weight equal to inverse of efficiency. We then repeat the process with the lowest estimate of efficiency and compute the quantity

$$
\frac{r_{\epsilon}-r_{0}}{r_{\epsilon}+r_{0}}
$$



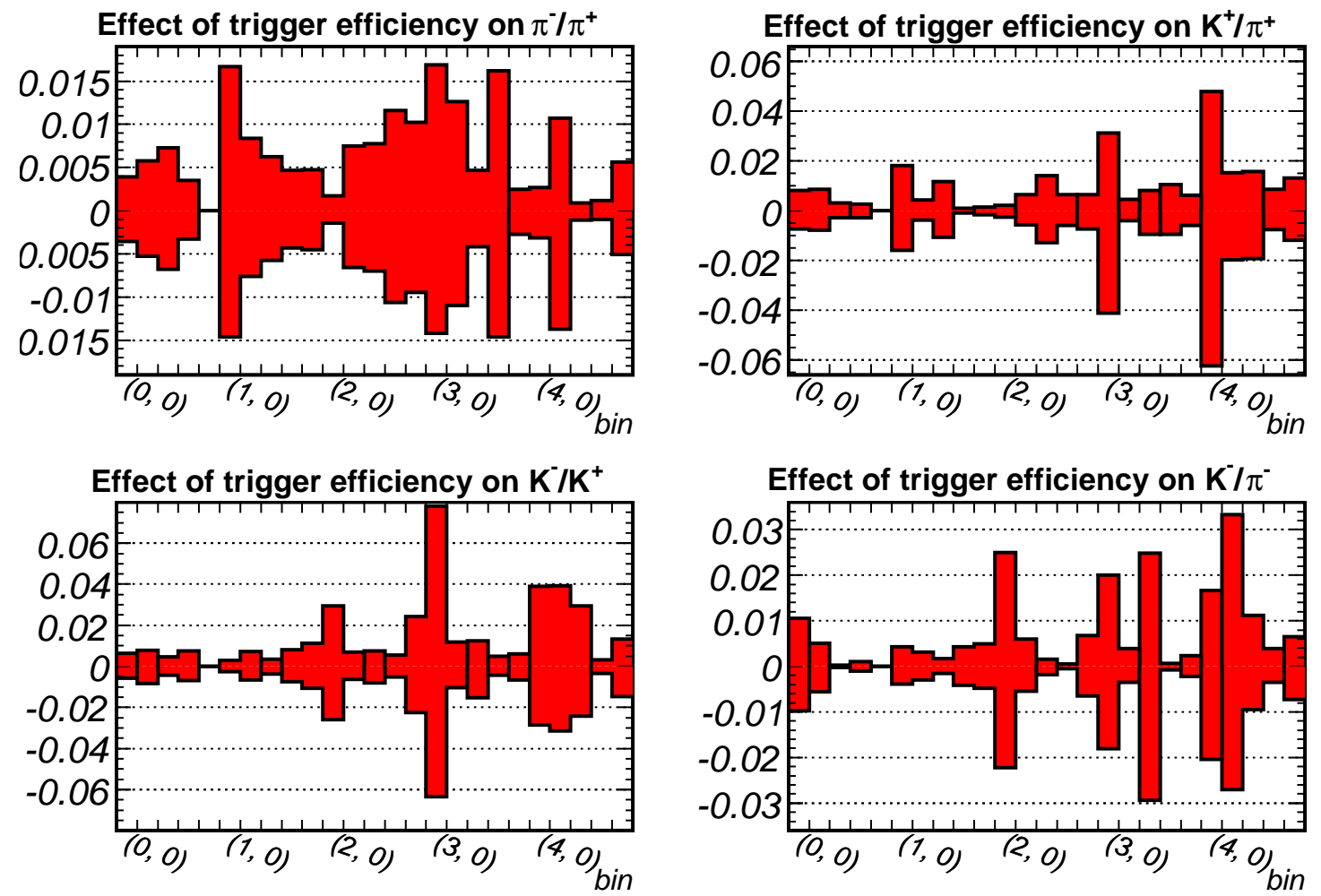

Figure 9.19: Effect of knowledge of interaction trigger efficiency on the ratios is quite small.

Here $r_{\epsilon}$ is the ratio measured given the assumption on trigger efficiency, and $r_{0}$ is the ratio derived using the average efficiency.

Another small error comes from the subtraction of signal associated with the particle production on the scintillator interaction trigger. The results of fits of Gaussian with exponential tails (Figure 9.8) give a 2.5\% uncertainty on target out subtraction. Figure 9.20 shows that even if we assume a $5 \%$ uncertainty on the fraction, this affects the ratios by less than $0.5 \%$.

The largest systematic error comes from modeling of background using the Monte Carlo. To compare how well data and Monte Carlo simulation agree, we normalize data and Monte Carlo particle ID distributions by the average number of pion events in the central two bins of the pion peak (see Figures 9.16 and 9.17). We then compute the amount of pion (kaon) background in those bins where pion (kaon) occupancy is non-zero ${ }_{\boldsymbol{k}}$ The ratio of data/Monte Carlo background, plotted in Figure 9.21, shows that in general the amount of background is larger in data than in Monte Carlo. This is in part due to the fact that 

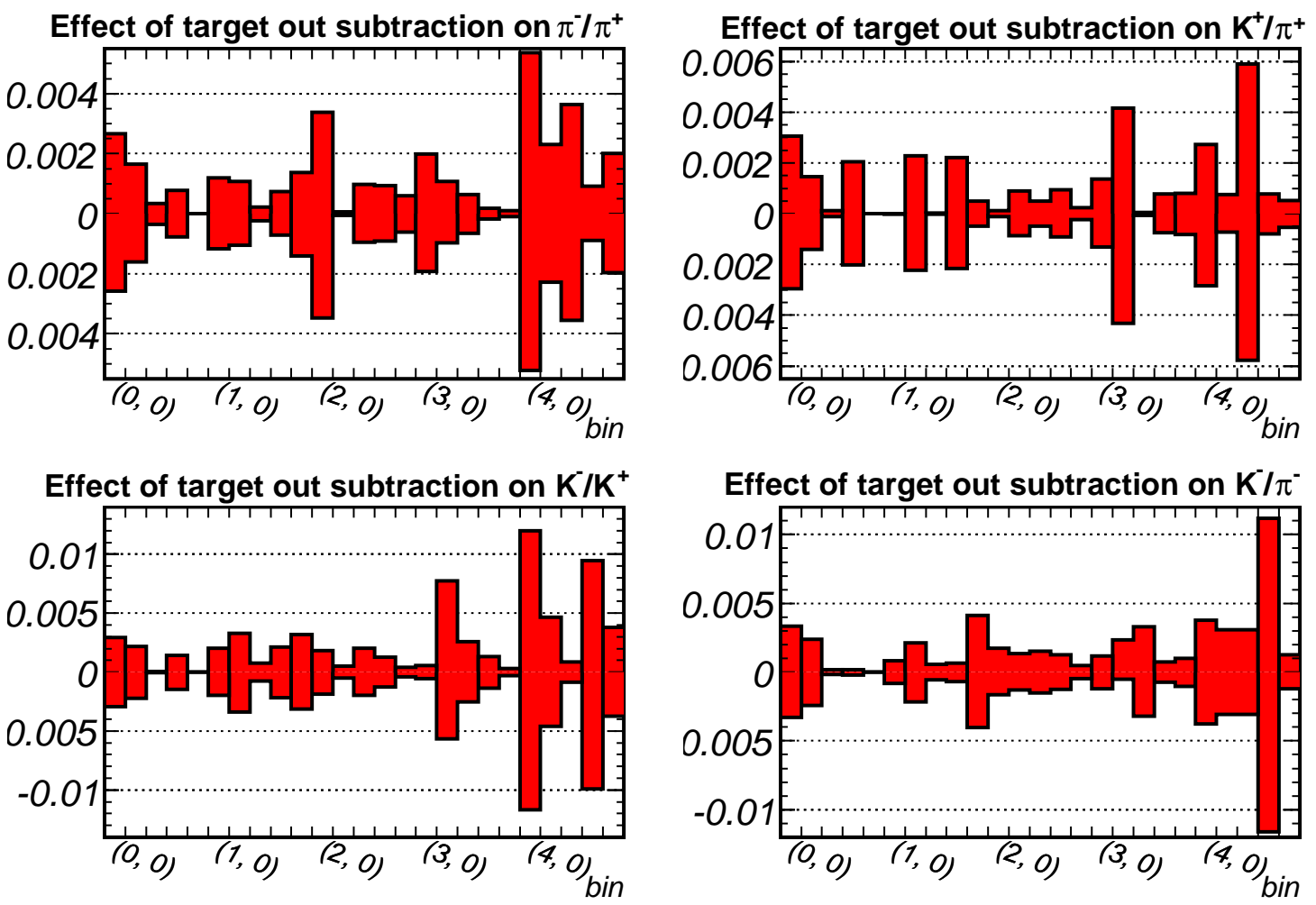

Figure 9.20: Effect on the ratio from $5 \%$ change in target out scale factor. 

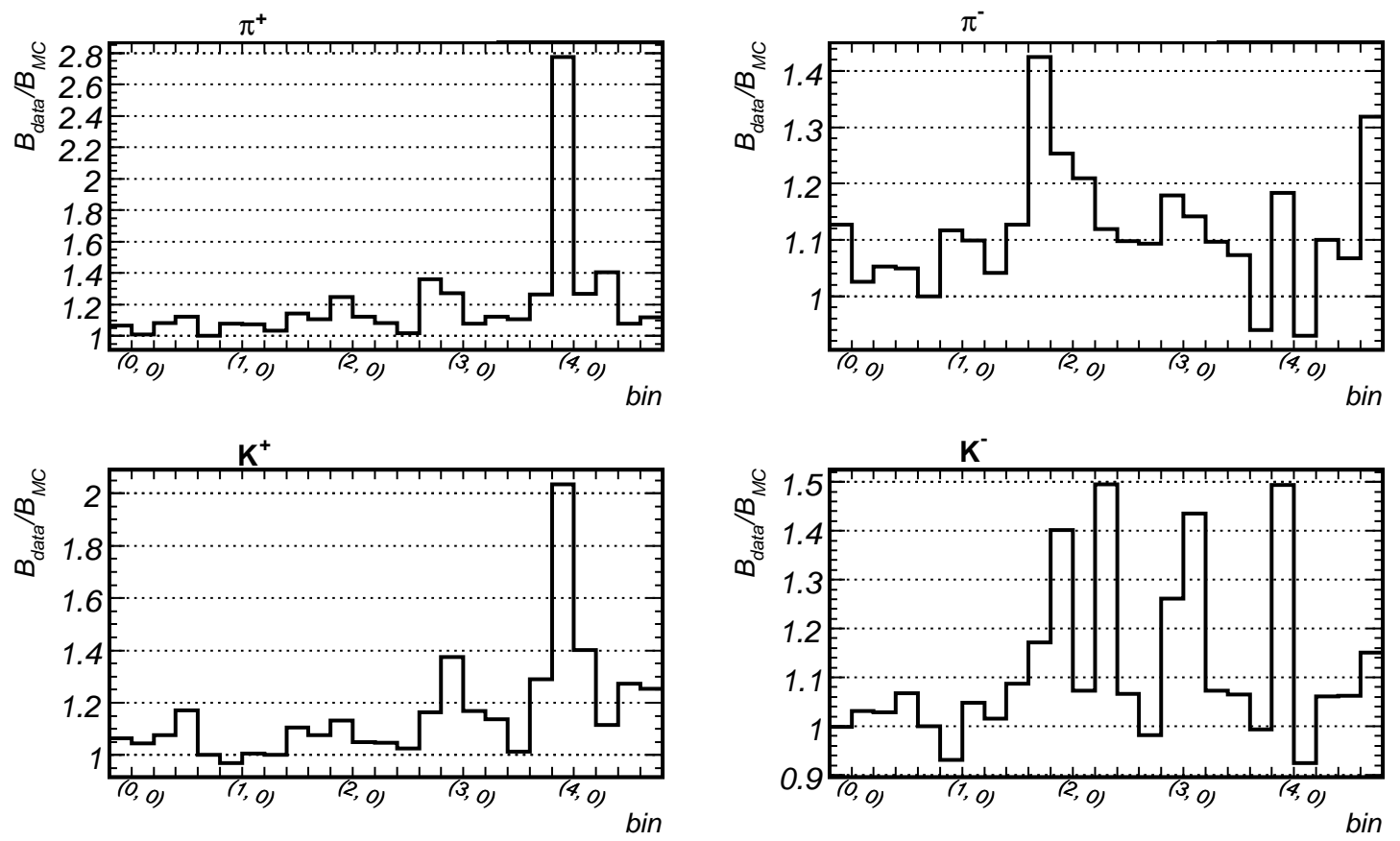

Figure 9.21: Data/Monte Carlo background ratio. In most bins, agreement between data and Monte Carlo is $10 \%$ or better. Bin $(4,0)$ has the largest proton background and is not well modeled by the Monte Carlo.

particle ID distributions are narrower in the Monte Carlo than in the data.

To judge the sensitivity of measured ratios to background modeling, we increase and decrease $(1-o)$ in the bins where we expect to find signal. The factor by which background is changed for each particle species is derived from the ratio of data/MC backgrounds, and is set to be at least 1.1 (the average of background ratios over all $\left(p_{z}, p_{T}\right)$ bins). We then plot the difference from ratios computed with nominal occupancy using Equation 9.4. Results shown in Figure 9.22 are consistent with expectations. The effect on the $\pi^{-} / \pi^{+}$ratio is small, since pion signal has little background. The larger effect on the $K^{-} / K^{+}$ratio stems in part from low statistics. By far the largest effect of background modeling is on the $K^{ \pm} / \pi^{ \pm}$ratios. The reason is that the level of background under the kaon peaks is significantly higher than under the pion peaks. Thus, decreasing $(1-o)$ by $10 \%$ can boost the ratio by up to $50 \%$. 

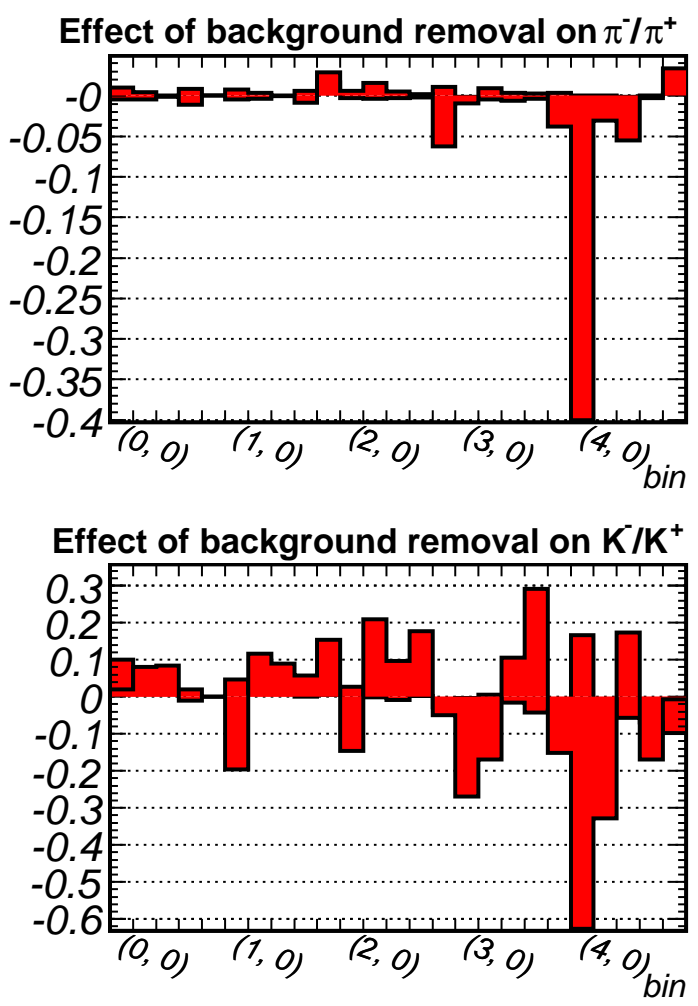

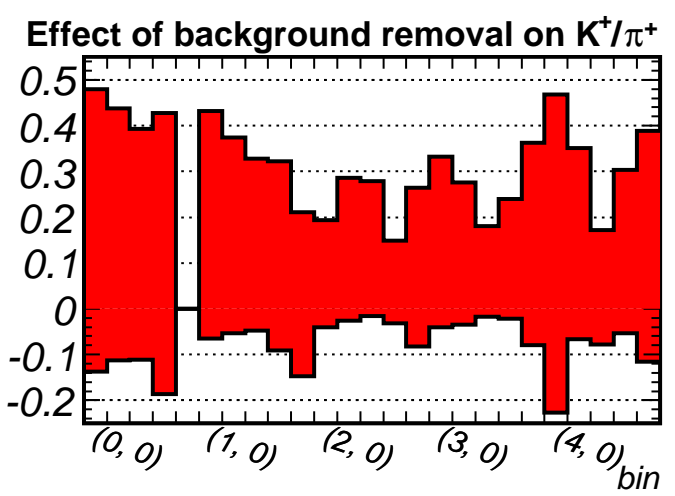

Effect of background removal on $\mathrm{K}^{-} / \pi^{-}$

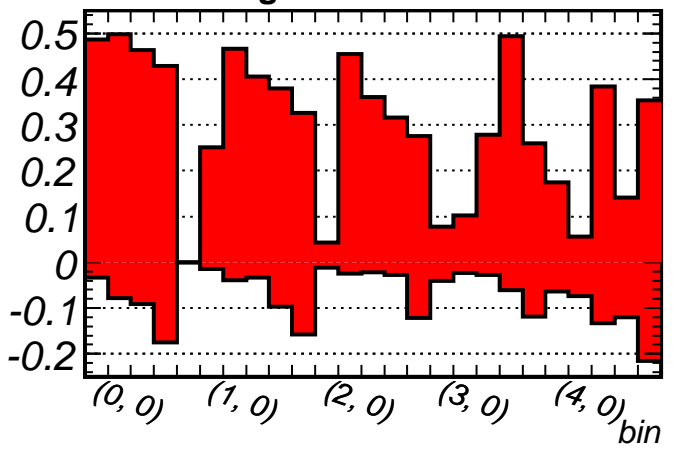

Figure 9.22: Systematic error from background subtraction. See text for explanation. 


\section{Chapter 10}

\section{Results}

\subsection{Production Ratios}

Equipped with Monte Carlo corrections and errors described in the previous chapter, we derive the ratios plotted in Figure 10.1 and listed in Tables 10.1, 10.2, 10.3, and 10.4. The ratios of like charges are consistent with FLUKA-06 predictions. However we find the predicted $\pi^{-} / \pi^{+}$and $K^{-} / K^{+}$ratios are higher by as much as $50 \%$ than the ratios derived in this measurement. The tendencies in both this measurement and FLUKA predictions are to decrease the $\pi^{-} / \pi^{+}$and $K^{-} / K^{+}$ratios with increasing $p_{z}$ and increase the ratios with increasing $p_{T}$, although with different slopes.

\subsection{Comparison to Existing Data}

Results presented here compare well with the existing data sets. For comparison, we define $x_{F}$ using the proton mass. The $\pi^{-} / \pi^{+}$ratio in our highest momentum bin (60$90 \mathrm{GeV} / c$ ) is significantly higher than the ratios derived from existing data. At this time, the reason for this discrepancy is not known ${ }_{\kappa}$

It is also interesting to compare our data to the BMPT parametrization of $400 \mathrm{GeV} / c$ protons on beryllium target[5]. Despite the fact that proton momentum is much higher, the parametrization does a good job describing behavior of ratios. The only exception is that in the parametrization $\pi^{-} / \pi^{+}$ratio is independent of $p_{T}$. This measurement and the ratio from NA49 indicates that this is not exactly so in the highest $p_{T}$ bin, which is not surprising as the model was derived using data with $p_{T} \leq 0.5 \mathrm{GeV} / c$. 

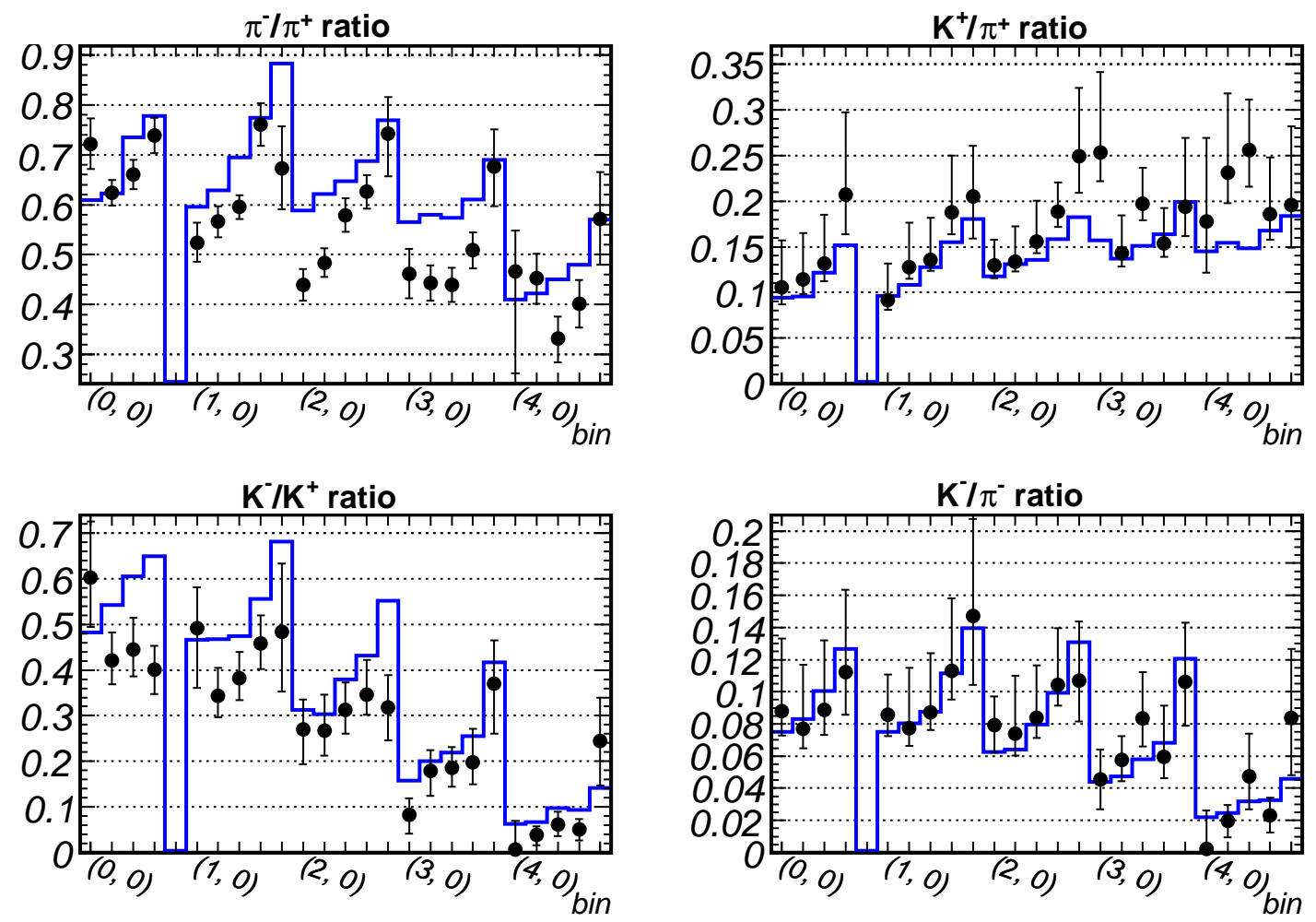

Figure 10.1: Measured ratios compared to particle production simulated with FLUKA-06 on $2 \%$ carbon target 


\begin{tabular}{|c|c|c|c|c|c|c|c|c|c|}
\hline Bin & 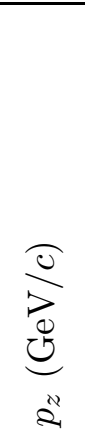 & $\begin{array}{l}\frac{0}{0} \\
\frac{0}{0} \\
\overrightarrow{2} \\
\overrightarrow{2}\end{array}$ & $\pi^{-} / \pi^{+}$ & 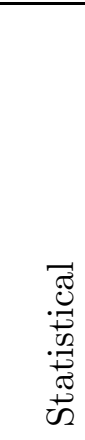 & 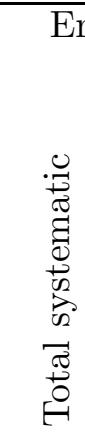 & 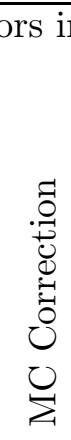 & 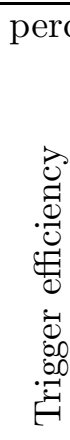 & 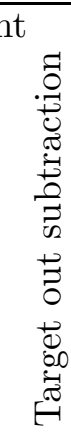 & 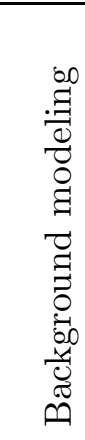 \\
\hline$(0,0)$ & 21.9 & 0.13 & $0.722_{-0.050}^{+0.051}$ & 5.2 & $\begin{array}{l}+4.8 \\
-4.7\end{array}$ & 4.6 & $\begin{array}{l}+0.4 \\
-0.4\end{array}$ & $\begin{array}{l}+0.3 \\
-0.3\end{array}$ & $\begin{array}{l}+1.0 \\
-0.5\end{array}$ \\
\hline$(0,1)$ & 21.9 & 0.30 & $0.624_{-0.025}^{+0.025}$ & 3.7 & $\begin{array}{l}+1.7 \\
-1.7\end{array}$ & 1.5 & $\begin{array}{l}+0.6 \\
-0.5\end{array}$ & $\begin{array}{l}+0.2 \\
-0.2\end{array}$ & $\begin{array}{l}+0.4 \\
-0.5\end{array}$ \\
\hline$(0,2)$ & 21.9 & 0.50 & $0.661_{-0.029}^{+0.029}$ & 3.9 & $\begin{array}{l}+2.0 \\
+2.0\end{array}$ & 1.9 & $\begin{array}{l}-0.7 \\
+0.7\end{array}$ & $\begin{array}{l}-0.0 \\
+0.0 \\
-0.0\end{array}$ & $\begin{array}{l}-0.0 \\
+0.0 \\
-0.1\end{array}$ \\
\hline$(0,3)$ & 22.0 & 0.74 & $0.739_{-0.036}^{+0.035}$ & 4.4 & $\begin{array}{l}+1.9 \\
-2.1\end{array}$ & 1.7 & $\begin{array}{l}-0.4 \\
+0.4 \\
-0.3\end{array}$ & $\begin{array}{l}-0.0 \\
+0.1\end{array}$ & $\begin{array}{l}-0.1 \\
+0.9 \\
-1.1\end{array}$ \\
\hline$(1,0)$ & 27.1 & 0.13 & $0.524_{-0.039}^{+0.039}$ & 4.9 & $\begin{array}{l}+5.7 \\
-5.6\end{array}$ & 5.3 & $\begin{array}{l}+1.7 \\
-1.5\end{array}$ & $\begin{array}{l}+0.1 \\
-0.1\end{array}$ & $\begin{array}{l}+0.8 \\
-0.5\end{array}$ \\
\hline$(1,1)$ & 27.1 & 0.30 & $0.566_{-0.031}^{+0.031}$ & 3.8 & $\begin{array}{l}-5.0 \\
+4.1 \\
-4.0\end{array}$ & 3.9 & $\begin{array}{l}-1.5 \\
+0.8 \\
-0.8\end{array}$ & $\begin{array}{l}-0.1 \\
+0.1 \\
-0.1\end{array}$ & $\begin{array}{l}-0.5 \\
+0.3 \\
-0.4\end{array}$ \\
\hline$(1,2)$ & 27.2 & 0.49 & $0.595_{-0.024}^{+0.024}$ & 3.8 & $\begin{array}{l}-1.0 \\
+1.4 \\
-1.4\end{array}$ & 1.3 & $\begin{array}{l}+0.6 \\
+0.6\end{array}$ & $\begin{array}{l}+0.0 \\
+0.0\end{array}$ & $\begin{array}{l}-0.4 \\
+0.0 \\
-0.0\end{array}$ \\
\hline$(1,3)$ & 27.3 & 0.75 & $0.761_{-0.043}^{+0.043}$ & 4.0 & $\begin{array}{r}+3.9 \\
-4.0\end{array}$ & 3.8 & $\begin{array}{l}-0.0 \\
+0.5 \\
-0.4\end{array}$ & $\begin{array}{l}-0.0 \\
+0.1 \\
-0.1\end{array}$ & $\begin{array}{l}-0.0 \\
+0.6 \\
-0.9\end{array}$ \\
\hline$(1,4)$ & 27.5 & 1.08 & $0.673_{-0.082}^{+0.084}$ & 11.8 & $\begin{array}{l}+4.2 \\
{ }_{-3.0}^{4}\end{array}$ & 3.0 & $\begin{array}{r}+0.5 \\
+0.4 \\
-0.4\end{array}$ & $\begin{array}{l}+0.1 \\
{ }_{-0.1}^{+}\end{array}$ & $\begin{array}{l}+2.9 \\
0.0\end{array}$ \\
\hline$(2,0)$ & 35.6 & 0.13 & $0.439_{-0.032}^{+0.032}$ & 6.2 & $\begin{array}{l}+3.8 \\
-3.7\end{array}$ & 3.7 & $\begin{array}{l}+0.2 \\
-0.1\end{array}$ & $\begin{array}{l}+0.3 \\
-0.3\end{array}$ & $\begin{array}{l}0.6 \\
-0.3\end{array}$ \\
\hline$(2,1)$ & 35.5 & 0.30 & $0.483_{-0.028}^{+0.029}$ & 4.4 & $\begin{array}{l}+4.0 \\
-3.7\end{array}$ & 3.6 & $\begin{array}{l}+0.7 \\
-0.7\end{array}$ & $\begin{array}{l}+0.0 \\
-0.0\end{array}$ & $\begin{array}{l}+1.6 \\
-0.4\end{array}$ \\
\hline$(2,2)$ & 35.6 & 0.50 & $0.579_{-0.033}^{+0.034}$ & 4.5 & $\begin{array}{l}+3.7 \\
+3.6\end{array}$ & 3.6 & $\begin{array}{l}+0.8 \\
-0.7\end{array}$ & $\begin{array}{l}+0.0 \\
-0.1\end{array}$ & $\begin{array}{l}+0.6 \\
+0.3\end{array}$ \\
\hline$(2,3)$ & 35.6 & 0.76 & $0.626_{-0.034}^{+0.034}$ & 4.5 & $\begin{array}{l}+3.0 \\
-3.0\end{array}$ & 2.8 & $\begin{array}{l}-0.2 \\
+1.2 \\
-1.1\end{array}$ & $\begin{array}{l}-0.1 \\
+0.1 \\
-0.1\end{array}$ & $\begin{array}{l}-0.3 \\
+0.2 \\
-0.2\end{array}$ \\
\hline$(2,4)$ & 36.0 & 1.19 & $0.743_{-0.086}^{+0.073}$ & 9.3 & $\begin{array}{l}+3.0 \\
-6.8\end{array}$ & 2.6 & $\begin{array}{l}+1.0 \\
+0.9\end{array}$ & $\begin{array}{l}+0.1 \\
-0.1\end{array}$ & $\begin{array}{l}+1.1 \\
+6.2 \\
-1.2\end{array}$ \\
\hline$\overline{(3,0)}$ & 48.6 & 0.13 & $0.462_{-0.050}^{+0.050}$ & 8.9 & $\begin{array}{l}+6.1 \\
+6.1 \\
-6.1\end{array}$ & 5.8 & $\begin{array}{l}\frac{-1.7}{+1.7} \\
-1.4\end{array}$ & $\begin{array}{l}\frac{-1 .}{70.2} \\
-0.2\end{array}$ & $\begin{array}{l}-0.2 \\
+-10 \\
-1.0\end{array}$ \\
\hline$(3,1)$ & 48.9 & 0.30 & $0.443_{-0.036}^{+0.036}$ & 6.4 & $\begin{array}{l}-0.1 \\
+5.1 \\
-50\end{array}$ & 4.8 & $\begin{array}{l}-1.4 \\
+1.3\end{array}$ & +0.1 & $\begin{array}{l}-1.0 \\
+1.0\end{array}$ \\
\hline$(3,2)$ & 49.2 & 0.50 & $0.439_{-0.034}^{+0.034}$ & 6.5 & $\begin{array}{l}-4.0 \\
+4.4 \\
-4.4\end{array}$ & 4.4 & $\begin{array}{l}-1.5 \\
+0.4 \\
-0.4\end{array}$ & $\begin{array}{l}+0.1 \\
+0.1 \\
-0.1\end{array}$ & $\begin{array}{l}+0.0 \\
+0.6\end{array}$ \\
\hline$(3,3)$ & 48.8 & 0.76 & $0.509_{-0.036}^{+0.036}$ & 5.9 & $\begin{array}{l}+4.4 \\
+3.9\end{array}$ & 3.6 & $\begin{array}{l}-1.6 \\
+1.5\end{array}$ & $\begin{array}{l}-0.0 \\
+0.0\end{array}$ & $\begin{array}{l}+0.0 \\
+0.4\end{array}$ \\
\hline$(3,4)$ & 49.0 & 1.25 & $0.676_{-0.079}^{+0.075}$ & 10.1 & $\begin{array}{l}+4.4 \\
-5.8\end{array}$ & 4.4 & $\begin{array}{l}+0.2 \\
+0.3\end{array}$ & $\begin{array}{l}+0.0 \\
+0.0\end{array}$ & $\begin{array}{l}+0.4 \\
-3.8\end{array}$ \\
\hline$(4,0)$ & 69.6 & 0.13 & $0.467_{-0.204}^{+0.082}$ & 17.2 & $\begin{array}{l}+3.8 \\
-40.3\end{array}$ & 3.8 & $\begin{array}{l}+0.3 \\
-0.3 \\
-0.3\end{array}$ & $\begin{array}{l}+0.5 \\
+0.5\end{array}$ & $\begin{array}{l}-0.0 \\
+40.1\end{array}$ \\
\hline$(4,1)$ & 69.1 & 0.30 & $0.452_{-0.051}^{+0.049}$ & 10.5 & $\begin{array}{r}-40.8 \\
+2.8 \\
-4.2\end{array}$ & 2.6 & $\begin{array}{l}-0.0 \\
+1.1 \\
-1.4\end{array}$ & $\begin{array}{l}-0.2 \\
+0.2 \\
-0.2\end{array}$ & $\begin{array}{r}-40.1 \\
+0.0 \\
-3.1\end{array}$ \\
\hline$(4,2)$ & 68.1 & 0.49 & $0.332_{-0.047}^{+0.041}$ & 12.9 & $\begin{array}{r}+2.8 \\
\end{array}$ & 2.8 & $\begin{array}{l}+0.1 \\
-0.1\end{array}$ & $\begin{array}{l}+0.4 \\
-0.4\end{array}$ & $\begin{array}{l}+0.0 \\
-5.5\end{array}$ \\
\hline$(4,3)$ & 68.8 & 0.77 & $0.402_{-0.047}^{+0.047}$ & 11.4 & $\begin{array}{l}-2.2 \\
+2.9 \\
-2.9\end{array}$ & 2.9 & $\begin{array}{l}+0.1 \\
+0.1\end{array}$ & $\begin{array}{l}-0.1 \\
+0.1\end{array}$ & $\begin{array}{l}-0.0 \\
+0.0 \\
-0.3\end{array}$ \\
\hline$(4,4)$ & 70.7 & 1.33 & $0.572_{-0.092}^{+0.094}$ & 15.8 & $\begin{array}{l}+4.6 \\
-3.2 \\
\end{array}$ & 3.1 & $\begin{array}{l}+0.6 \\
-0.5\end{array}$ & $\begin{array}{l}+0.2 \\
-0.2\end{array}$ & $\begin{array}{l}+3.4 \\
0.0\end{array}$ \\
\hline
\end{tabular}

Table 10.1: $\pi^{-} / \pi^{+}$production ratio and errors. Listed $p_{z}$ and $p_{t}$ are computed with $\pi^{+}$ spectrum. 


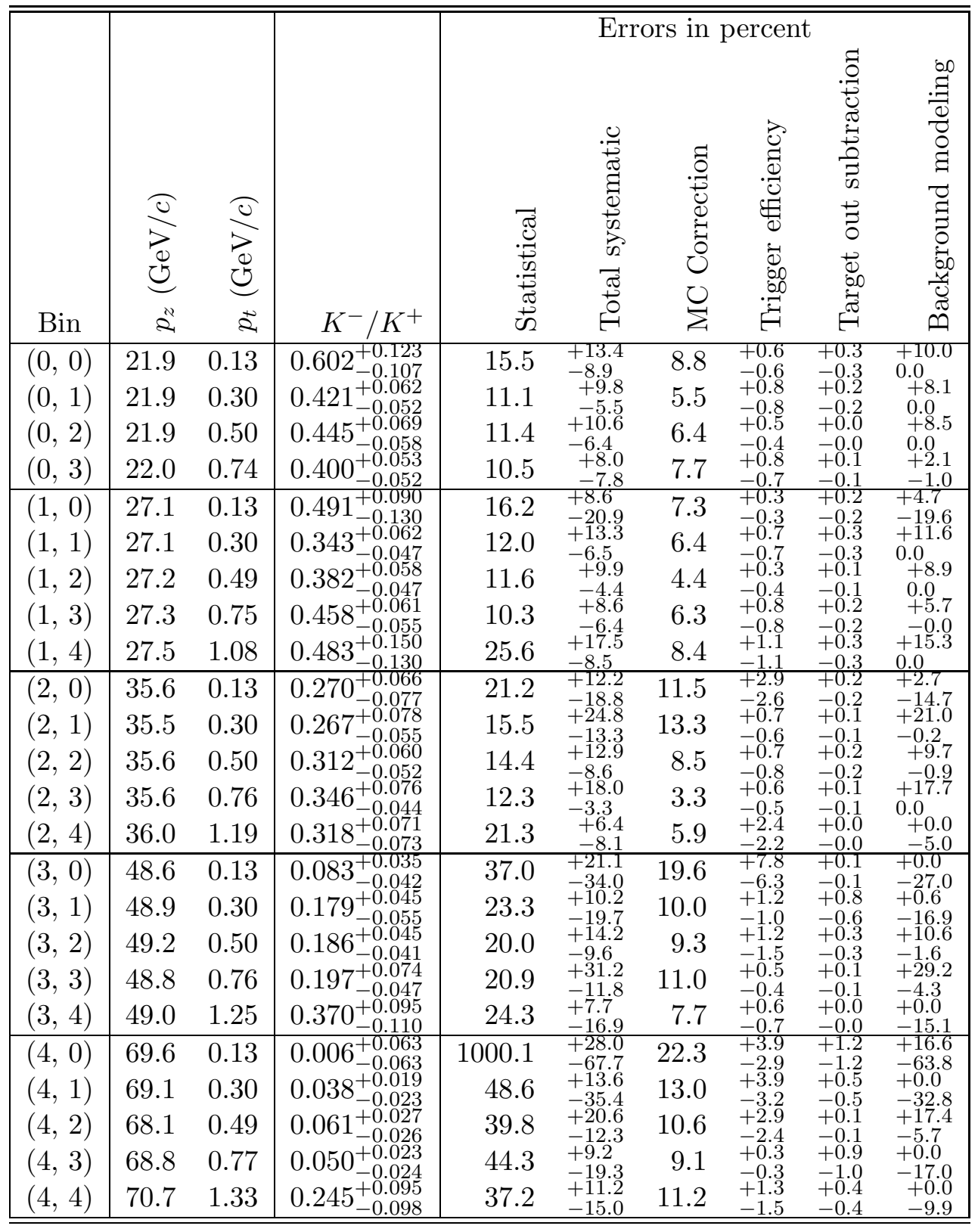

Table 10.2: $K^{-} / K^{+}$production ratio and errors. Listed $p_{z}$ and $p_{t}$ are computed using $\pi^{+}$ spectrum. 


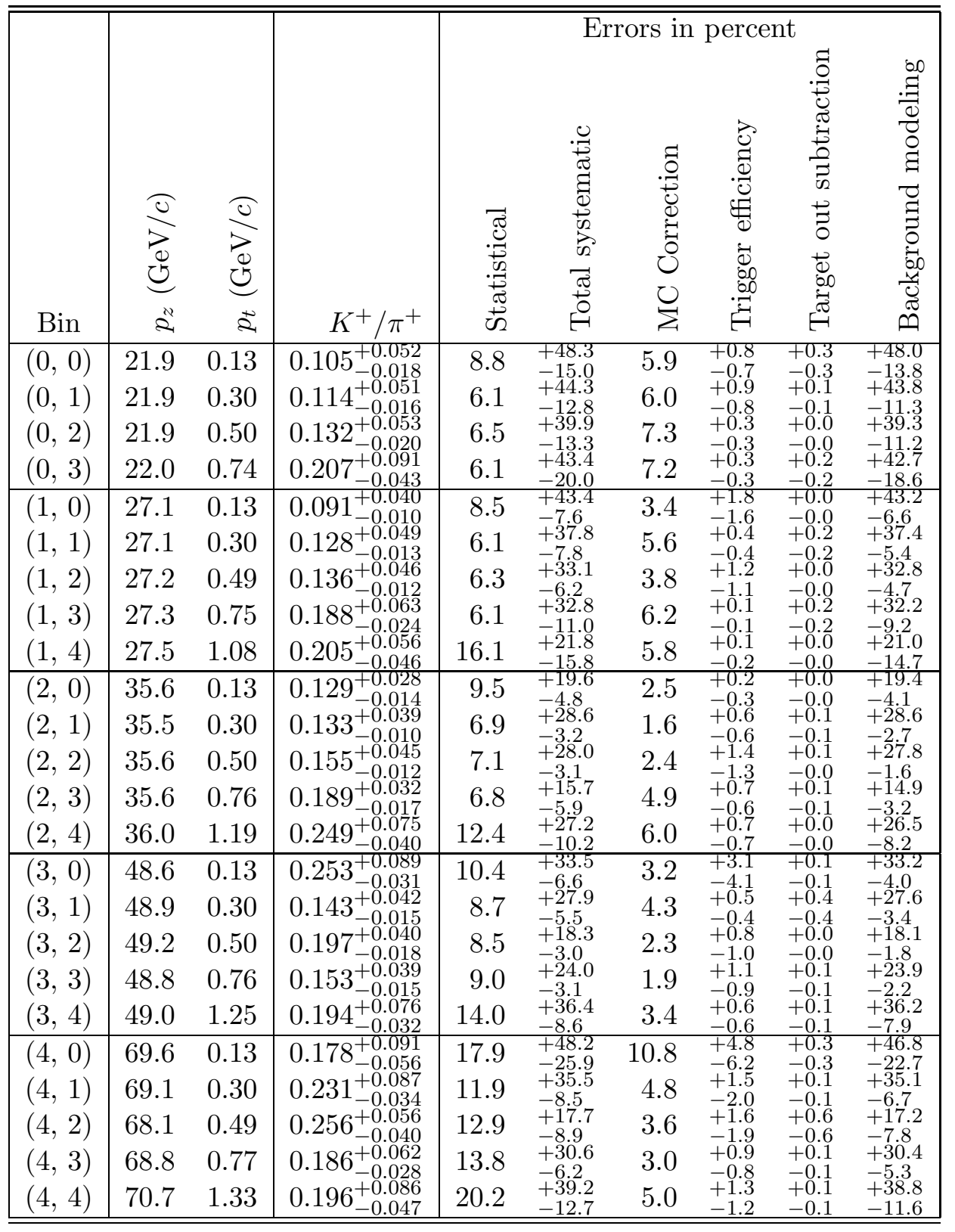

Table 10.3: $K^{+} / \pi^{+}$production ratio and errors. Listed $p_{z}$ and $p_{t}$ are computed with $\pi^{+}$ spectrum. 


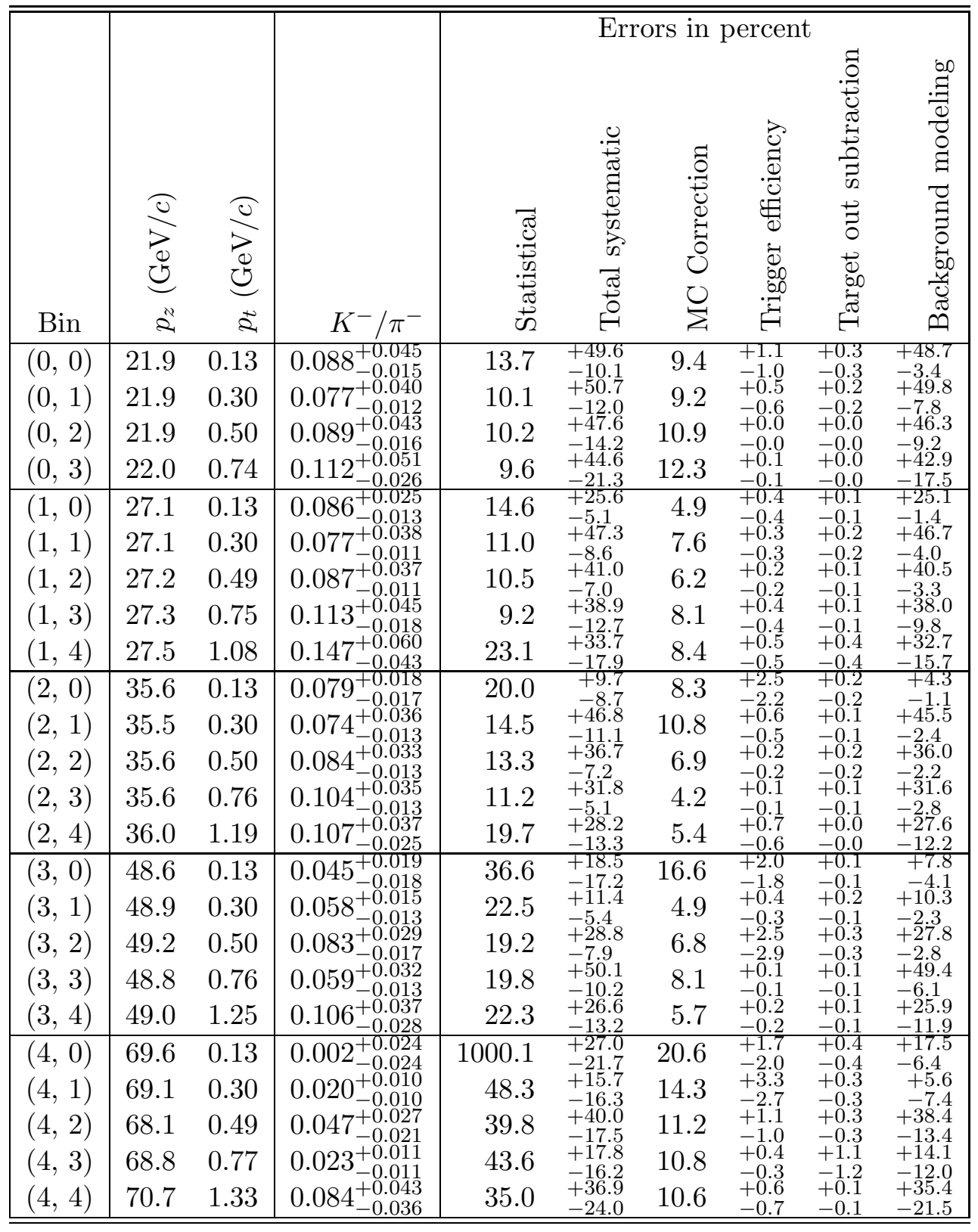

Table 10.4: $K^{-} / \pi^{-}$production ratio and errors. Listed $p_{z}$ and $p_{t}$ are computed with $\pi^{+}$ spectrum. 

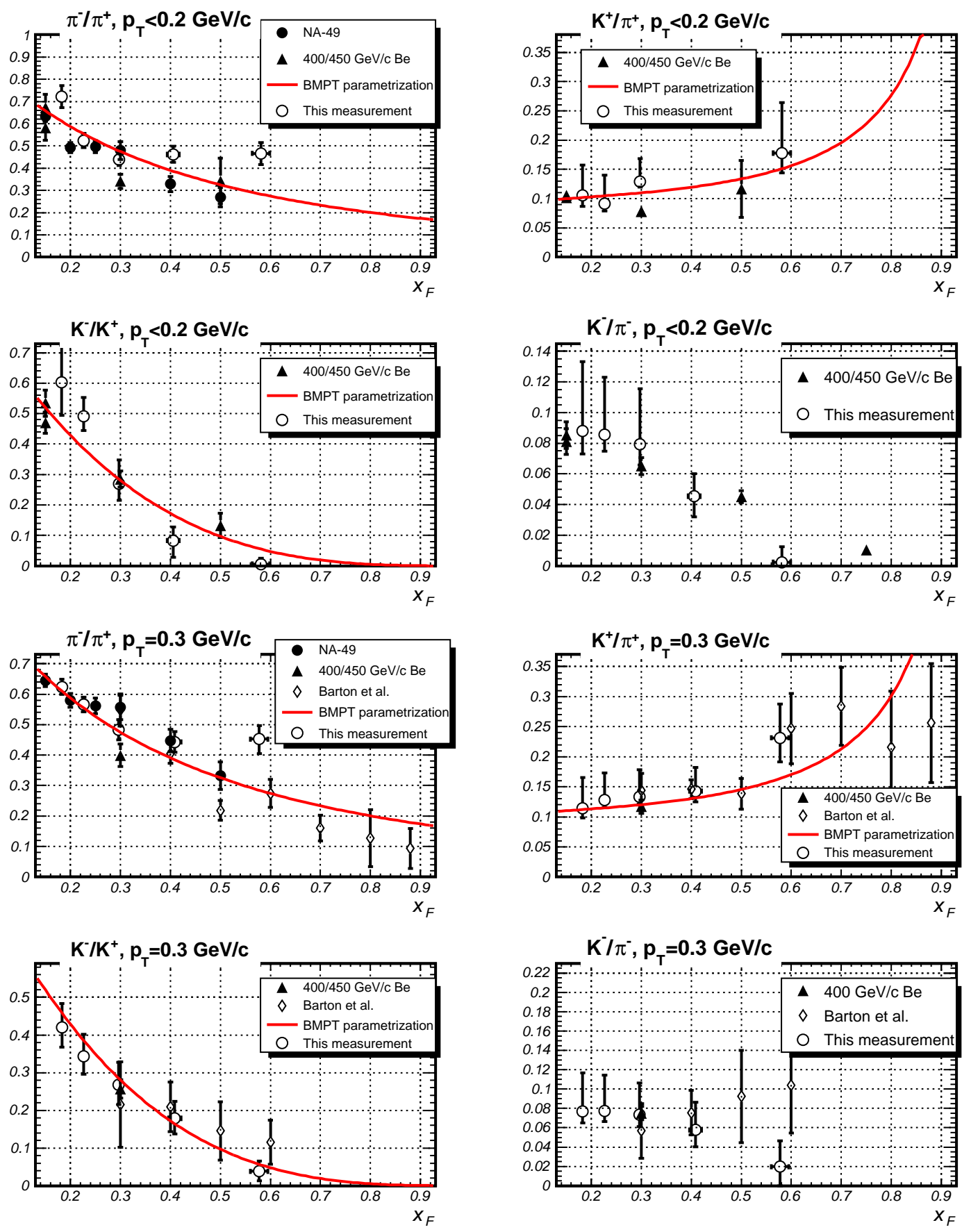

Figure 10.2: Comparison to existing measurements and parametrization of beryllium data. 

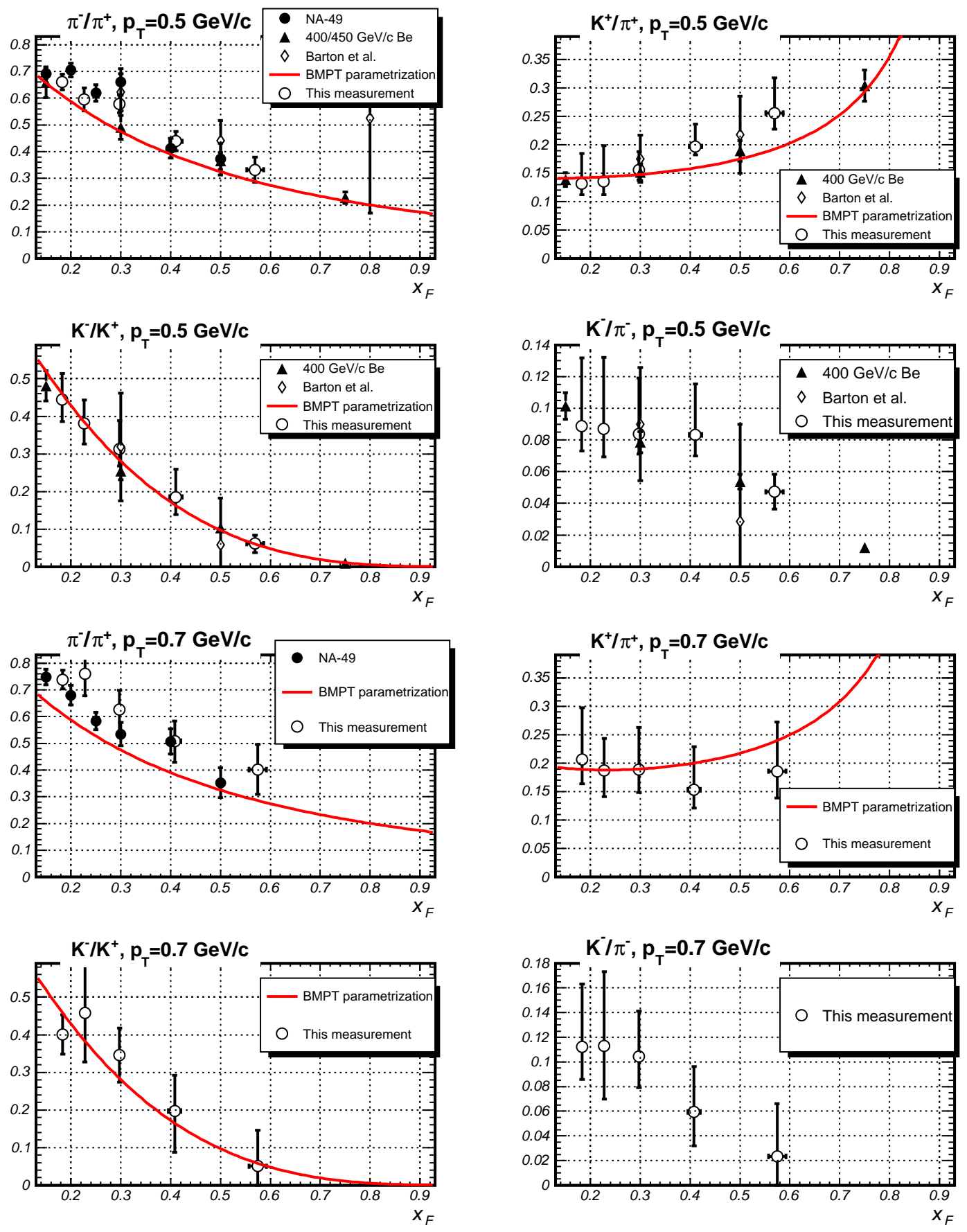

Figure 10.3: Comparison to existing measurements and parametrization of beryllium data. 


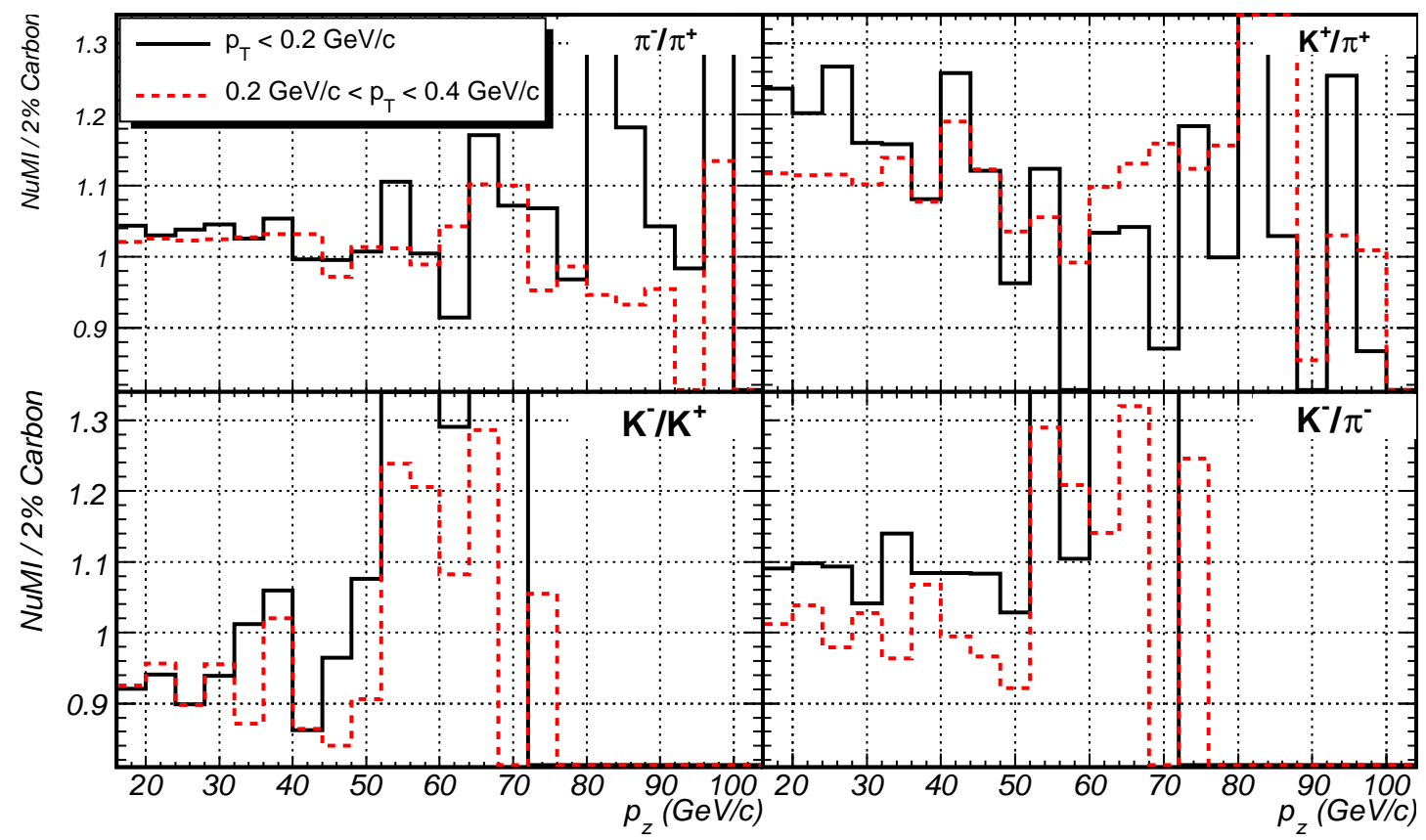

Figure 10.4: Comparison of the ratios on the NuMI target and the $2 \%$ target. The NuMI ratios are generated with FLUKA-05, $2 \%$ ratios are generated with FLUKA-06.

\subsection{Comparison to MINOS Measurements}

It is interesting to compare this measurement to the particle production ratios on the NuMI target. Figure 10.4 shows that the thin target ratios and NuMI target ratios predicted by FLUKA are similar to about $10 \%$. This is expected as production of particle at high momenta is dominated by interactions of primary protons with carbon.

MINOS beam systematics group takes advantage of the fact that the MINOS neutrino flux spectrum can be changed significantly by varying the target position with respect to the two focusing horns and the horn currents.[28, 16] Variation of target position with respect to the horns changes longitudinal momentum of focused hadrons and variation of horn currents changes their transverse momentum. The near detector has large neutrino rate, allowing the group to "effectively map out particle production in $\left(x_{F}, p_{T}\right)$ ".

The ratios derived from the fitted MINOS hadronic spectrum nicely compare to the measurement presented here (see Figure 10.5). 

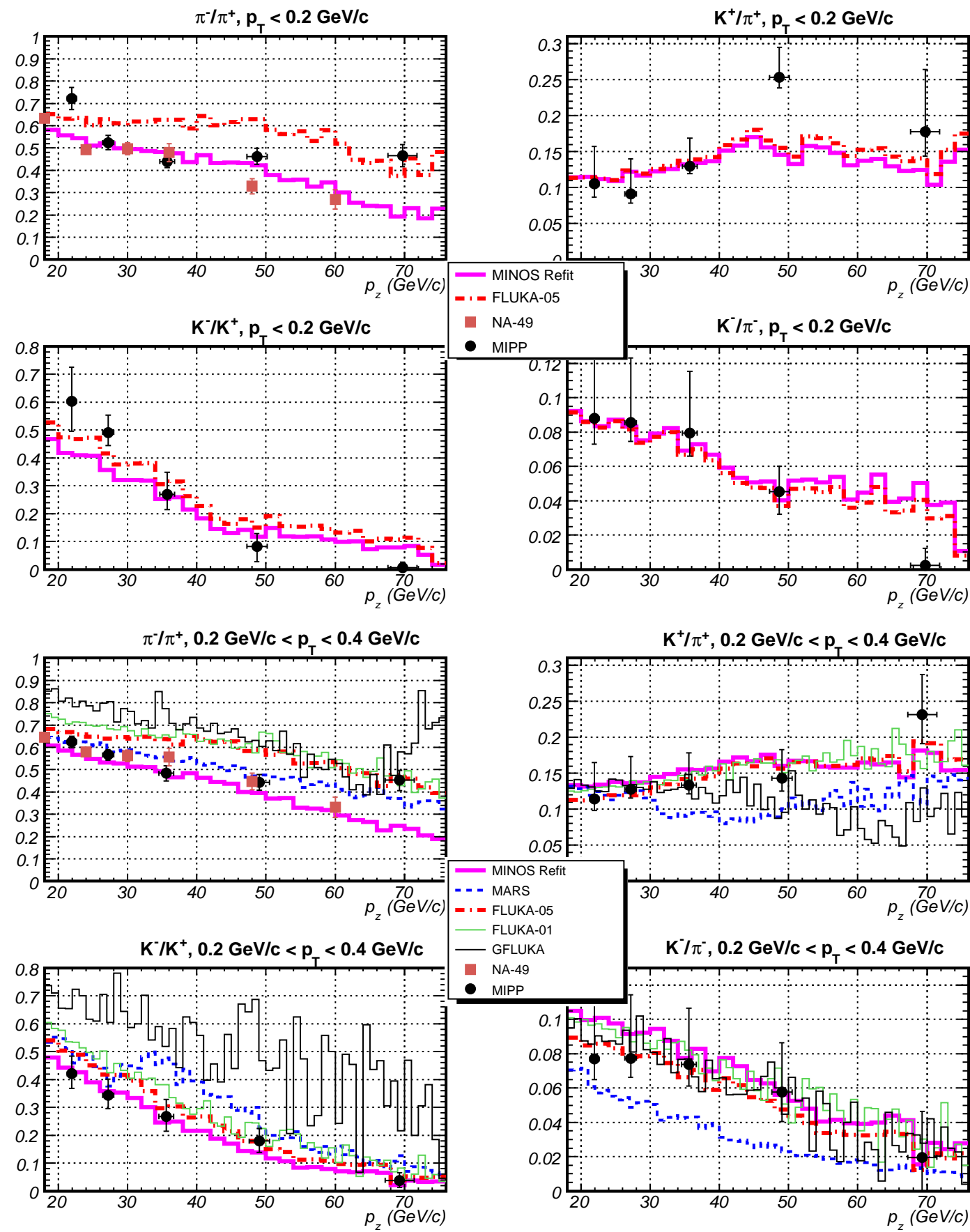

Figure 10.5: Comparison to various Monte Carlo models and MINOS fitted ratios. The Monte Carlo and fitted ratios have been generated with the MINOS target, while the measured ratios come from the thin target data. For models other than FLUKA-05 and MINOS, the ratios are computed for $p_{T}<1 \mathrm{GeV} / c$ only, so they are compared to the bins which have the largest statistics in $p_{T}$. 


\subsection{Discussion and Outlook}

We presented the first measurement using the data from the MIPP spectrometer. The precision of the measurement is generally limited by statistics, although at this time our understanding of the background is limited and is a significant source of systematic error, especially for kaons. Better modeling of the RICH response in the Monte Carlo simulation will certainly reduce the systematic errors associated with the measurement.

The measurement is consistent with previously published data, except in the highest momentum bin where we (apparently) find find too few $\pi^{+}$. The bins with high $p_{z}$ have smaller pion and kaon statistics, therefore good knowledge of background is required to interpret the data correctly. Good agreement is also found between the ratios presented here and the ratios derived from the fitted MINOS pion and kaon spectra.

We do find inconsistencies with the FLUKA-06 Monte Carlo spectrum, especially in the $\pi^{-} / \pi^{+}$ratio. Addressing these discrepancies between the model and existing data will be extremely valuable for present and future neutrino experiments. 


\section{Bibliography}

[1] H. W. Atherton et al. Precise measurements of particle production by 400-gev/c protons on beryllium targets. 1980. CERN-80-07.

[2] D. S. Barton et al. Experimental study of the A-dependence of inclusive hadron fragmentation. Phys. Rev., D27:2580-2599, 1983.

[3] Gerard Beekmans. Linux From Scratch Version 4.0-RC1. Linux From Scratch Project, 2002.

[4] Fred Bieser, Ron Jones, , and Charles McFarland. Data links for the EOS TPC. IEEE Trans. Nucl. Sc., 38:335-336, 1991.

[5] M. Bonesini, A. Marchionni, F. Pietropaolo, and T.Tabarelli de Fatis. On particle production for high energy neutrino beams. Eur.Phys.J., C20:13-27, 2001.

[6] C. Bower, N.Graf, M. D. Messier, and J. Paley. The Indiana trigger counter. MIPP Note, 55, 2005.

[7] R. A. Burnstein et al. Hypercp: A high-rate spectrometer for the study of charged hyperon and kaon decays. Nucl. Instrum. Meth., A541:516-565, 2005.

[8] M. G. Catanesi et al. Proposal for hadron production measurements using the na49 detector for use in long-baseline and atmospheric neutrino flux calculations. 2001. CERN-SPSC-2001-017.

[9] CERN, Geneva, Switzerland. GEANT - Detector Description and Simulation Tool, 1993.

[10] D. C. Christian et al. High rate drift chambers. Nucl. Instrum. Meth., A345:62-71, 1994.

[11] SPY Collaboration. Eur. Phys. J. C, 10:605, 1999.

[12] Creative Electronic Systems S.A. CBD 8210 CAMAC Branch Driver, July 1996.

[13] M. De Palma et al. A system of large multiwire proportional chambers for a high intensity experiment. Nucl. Instr. Meth., 217:135, 1983.

[14] W.N. English and G.C. Hanna. Canadian Journal of Physics, 31:768, 1953. 
[15] C. Alt et al. Inclusive production of charged pions in $\mathrm{p}+\mathrm{C}$ collisions at $158 \mathrm{GeV} / c$ beam momentum. arXiv:hep-ex/0606028, 2006.

[16] D. G. Michael et al. Observation of muon neutrino disappearance with the MINOS detector in the NuMI neutrino beam. Phys. Rev. Lett., 97, 2006.

[17] G. Rai et al. A TPC detector for the study of high multiplicity heavy ion collisions. IEEE Trans. Nucl. Sc., 37:56-64, 1990.

[18] H. Wieman et al. A TPC detector for the study of high multiplicity heavy ion collisions. Nucl. Phys., A525:617-620, 1991.

[19] J. Engelfried et al. The E781 (SELEX) RICH detector). Nucl. Inst. and Meth., A409:439-442, 1998.

[20] J. Engelfried et al. The RICH detector of the SELEX experiment. Nucl. Inst. and Meth., A433:149-152, 1999.

[21] J. Engelfried et al. The SELEX phototube RICH detector. Nucl. Inst. and Meth., A431:53-69, 1999.

[22] K. L. Lee et al. Analog-to-digital conversion using custom CMOS analog memory for the EOS time projection chamber. IEEE Trans. Nucl. Sc., 38:344-347, 1991.

[23] A. Fasso', A. Ferrari, J. Ranft, and P.R. Sala. Fluka: a multi-particle transport code. CERN 2005-10, INFN/TC_05/11, SLAC-R-7773, 2005.

[24] A. Fasso', A. Ferrari, S. Roesler, P.R. Sala, G. Battistoni, F. Cerutti, E. Gadioli, M.V. Garzelli, F. Ballarini, A. Ottolenghi, A. Empl, and J. Ranft. The physics models of fluka: status and recent developments. Computing in High Energy and Nuclear Physics Conference, 2003.

[25] Y. Fukuda et al. Evidence for oscillation of atmospheric neutrinos. Phys. Rev. Lett., 81:1562-1567, 1998.

[26] http://www.linuxfromscratch.org.

[27] Stuart A. Kleinfelder. A 4096 cell switched capacitor analog waveform storage integrated circuit. IEEE Trans. Nucl. Sc., 37:1230-1236, 1990.

[28] Sacha Kopp, Žarko Pavlović, Patricia Vahle, and Rustem Ospanov. Constraining the beam neutrino mc flux using the minos nd data. minos-doc-2965, April 2007.

[29] A. Lebedev. Chamber tuning and alignment. MIPP Note, 129, 2007.

[30] Valeri Lebedev. http://fnal.gov/optim.

[31] J. B. Lindsay, C. Millerin, J. C. Tarlé, H. Verveij, and H. Wendler. A fast and flexible data acquisition system for multiwire proportional chambers and other detectors. Nucl. Inst. and Meth., 156:329-333, 1978. 
[32] MINER $\nu \mathrm{A}$ Collaboration. The MINER $\nu \mathrm{A}$ technical design report, 2006.

[33] Te MiniBooNE collaboration. A search for electron neutrino appearance at the $\delta m^{2} \sim$ $1 \mathrm{ev}^{2}$ scale. arXiv:hep-ex/0704.1500, 2007.

[34] N. Mokhov. MARS code system. http://www-ap.fnal.gov/MARS/.

[35] M. Nakamura, D.A. Landis, and G. Rai. Low power shaper amplifier for the EOS TPC detector. IEEE Trans. Nucl. Sc., 38:50-52, 1991.

[36] J. Paley, R. Soltz, P.D. Barnes, M. Heffner, J. Klay, and D. Miller. MIPP TPC reconstruction. MIPP Note, 129, 2006.

[37] Anna Peisert and Fabio Sauli. Drift and diffusion of electrons in gases: a compilation. Technical Report 84-08, CERN, July 1984.

[38] S.F.Biagi. Magboltz 2. http://ref.web.cern.ch/ref/CERN/CNL/2000/001/magboltz.

[39] S.F.Biagi. Monte Carlo simulation of electron drift and diffusion in counting gases under the influence of electric and magnetic fields. Nucl. Inst. and Meth., A421:234240, 199.

[40] Ron Soltz. Private communication.

[41] M. Staric and P. Krizan. An iterative method for the analysis of Cherenkov rings in the HERA-B RICH. Nucl. Inst. and Meth., A 433:279-285, 1999.

[42] H. Wieman and the EOS Collaboration. Heavy ion reaction measurements with the EOS TPC (looking for central collisions with missing energy). In Proceedings of the Tenth Winter Workshop on Nuclear Dynamics (Snowbird, Utah), January 14-21, 1994.

[43] Xihong Yang. Centrality dependence of strangeness production in proton-nucleus collisions at AGS energies. PhD thesis, Columbia University, 2000. 